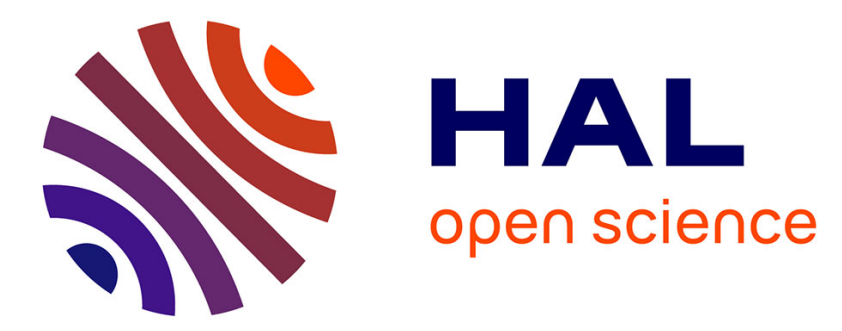

\title{
Pierres gravées de la Marche à Lussac-les-Châteaux (Vienne) : techniques, technologie et interprétations
}

\author{
Nicolas Mélard
}

\section{To cite this version:}

Nicolas Mélard. Pierres gravées de la Marche à Lussac-les-Châteaux (Vienne) : techniques, technologie et interprétations. Gallia Préhistoire - Préhistoire de la France dans son contexte européen, 2008, 50, pp.143-268. 10.3406/galip.2008.2460 . hal-02343321

\section{HAL Id: hal-02343321 \\ https://hal.science/hal-02343321}

Submitted on 19 Dec 2019

HAL is a multi-disciplinary open access archive for the deposit and dissemination of scientific research documents, whether they are published or not. The documents may come from teaching and research institutions in France or abroad, or from public or private research centers.
L'archive ouverte pluridisciplinaire HAL, est destinée au dépôt et à la diffusion de documents scientifiques de niveau recherche, publiés ou non, émanant des établissements d'enseignement et de recherche français ou étrangers, des laboratoires publics ou privés.

\section{(1) (1) $\$$}

Distributed under a Creative Commons Attribution - NonCommercial - NoDerivatives $\mid 4.0$ 


\title{
PIERRES GRAVÉES DE LA MARCHE À LUSSAC-LES-CHÂTEAUX (VIENNE)
}

\section{Techniques, technologie et interprétations}

\author{
Nicolas MÉLARD*
}

\begin{abstract}
Mots-clés. Fonctions, gravures, Magdalénien moyen, microtopographie, 3D.
Résumé. Les créations artistiques du Magdalénien sont multiples et étonnamment riches. Dans le domaine de l'art mobilier, les pierres gravées jouent un rôle important pour l’iconographie de cette époque et reflètent ainsi de manière très représentative la diversité et la complexité des représentations magdaléniennes. Le site de la Marche, à Lussac-les-Châteaux (Vienne), daté du Magdalénien moyen, est depuis sa découverte connu pour ses pierres gravées; plusieurs chercheurs se sont essayés à leur déchiffrement et ont pu démontrer la présence de centaines de figurations. Des découvertes récentes ont livré plus d’un millier de nouvelles pièces gravées. Le but de cette étude est de caractériser ces supports, les gravures et les autres traces qui s’y trouvent. Mis à part la découverte de nouvelles figurations gravées, il apparaît en effet que ces pierres peuvent être considérées comme des témoins des comportements des Magdaléniens ainsi que de leur pensée symbolique. Enfin, la mise à l'épreuve de nouveaux moyens d'investigation dans le cadre d'une méthodologie adaptée aux supports montre son intérêt pour la compréhension, la valorisation et la conservation des objets préhistoriques.
\end{abstract}

Key-words. Function, engraving, Middle Magdalenian, microtopography, 3D.

Abstract. The artistic creations of Magdalenian peoples are multiple and astonishingly rich. In the domain of portable art, engraved stones play an important role in the iconography of this period and clearly reflect the diversity and complexity of Magdalenian representations. Since its discovery, the site of la Marche (Lussac-les-Châteaux, Vienne), dated to the Middle Magdalenian, has been known for its engraved stones; several researchers have attempted to decode them and have revealed the presence of hundreds of human and animal figurations. During a recent field project, more than one thousand new engraved stone fragments were discovered. The objective of the study presented here is to analyse these engravings and their supports, as well as other traces found on them. In addition to the discovery of new engraved figures, it appears that these stones can provide evidence of Magdalenian behaviours and activities, as well as their symbolic concepts. The testing of new investigative techniques in the context of a methodology adapted to these supports shows their value for the understanding, valorization and conservation of these prehistoric objects.

Translation: Magen O'FARRELL

Schlüsselwörter. Funktionen, Gravierungen, mittleres Magdalénien, Mikrotopographie, Plakettenkunst, 3D.

Zusammenfassung. Die künstlerischen Kreationen des Magdalénien sind vielfältig und erstaunlich reich an Bildern und Technicken. Im Bereich der Kleinkunst spielen die gravierten Steine (Plakettenkunst) eine bedeutende Rolle für die Ikonographie dieser Zeit und spiegeln so auf sehr repräsentative Weise die Vielfalt und Komplexität der Darstellungen des Magdalénien wider.

Der in das mittlere Magdalénien (14 000 B.P.) datierte Fundplatz la Marche (Lussac-les-Châteaux, Vienne) ist seit seiner Entdeckung für seine gravierten Steine bekannt; mehrere Forscher haben sich mit deren Entschlüsselung befasst und hunderte von figürlichen Darstellungen von Tieren und Menschen nachgewiesen. Bei neueren Forschungen wurden über tausend neue gravierte Steine entdeckt. Ziel dieser Arbeit war es, diese Objekte sowie die Gravierungen und die anderen Spuren auf den Oberflächen zu charakterisieren. Abgesehen von der Entdeckung neuer gravierter, figürlicher und abstrakter Darstellungen zeigt sich in der Tat, dass diese Steine durch die Spurenanalyse zu Zeugen für die Verhaltensweisen der Menschen des Magdalénien sowie deren symbolischen Denkens werden. Die Erprobung und Anwendung neuer, nicht invasiver Untersuchungsméthoden im Rahmen einer den Objekten angepassten Methodologie zeugt schließlich von der Effizienz, neue Untersuchungsmethoden im Bereich der Analyse und Konservierung anzuwenden.

Übersetzung: Nicolas MÉLARD

\footnotetext{
* Élève conservateur, Institut national du Patrimoine, 2 rue Vivienne, F-75002 Paris. Courriel : nicolas.melard@inp.fr
} 
L'époque magdalénienne a constitué, au sein du Paléolithique supérieur, un temps d'intense foisonnement artistique. Les hommes qui ont vécu durant cette période ont laissé un nombre considérable de vestiges d'art pariétal mais aussi d'art mobilier. Ces derniers possèdent des formes diverses et variées: outre des parures, des outils décorés ainsi que des sculptures en matières dures animales, les Magdaléniens ont produit des représentations sur pierres, sous forme de gravures. Des milliers de ces objets ont été trouvés dans des grottes, des abris ou encore en plein air.

L'iconographie des pierres gravées est elle-même très riche: figurations animales mais aussi humaines et abstraites apparaissent régulièrement sur ces pièces. Cependant, force est de constater que l'ensemble des traits et traces présents sur ces supports rendent généralement l'analyse, voire le simple déchiffrement, difficiles.

Ce constat est particulièrement valable pour les pierres gravées de la Marche. Ce site d'habitat est en effet célèbre pour le nombre, la richesse et la diversité de ses gravures. Plus de 2000 pierres gravées ont à ce jour été découvertes. Il semble par ailleurs qu'elles aient servi à des activités artistiques aussi bien qu'aux tâches quotidiennes. Ceci constitue à la fois un atout pour la recherche, dans le sens où elles deviennent par là même des témoins complets de la vie sur le site au Magdalénien, mais aussi une difficulté, puisque la multiplicité des usages doit être retrouvée, distinguée et identifiée; ce n'est en effet qu'à cette condition que l'interprétation devient possible.

Cet article est une synthèse du travail mené dans le cadre de notre thèse de doctorat (Mélard, 2006b). Dans ce projet, il ne s'agissait pas de constituer un catalogue exhaustif des pierres inédites de la grotte de la Marche mais d'élaborer un questionnement sur les pierres gravées de la Marche qui soit le plus global possible.

Grâce à cela et au protocole d'analyses envisagé, nous espérons apporter ici un certain nombre de réponses qui permettront, au travers des pierres gravées, de mieux percevoir ce qu'était la vie à la Marche au Magdalénien. Ces pierres constituent donc le prisme par lequel nous pensons pouvoir accéder à une meilleure compréhension du site et replacer celui-ci dans un contexte chrono-culturel plus général. Que représentaient ces pierres gravées pour les hommes préhistoriques? Comment étaient-elles employées? Quel(s) statut(s) et quel(s) rôle(s) tenaient plus précisément les gravures? Autant de questions qui guideront notre étude.

Reste à dire que cette étude représente, par sa démarche même, un exemple de l'importance de la transdisciplinarité

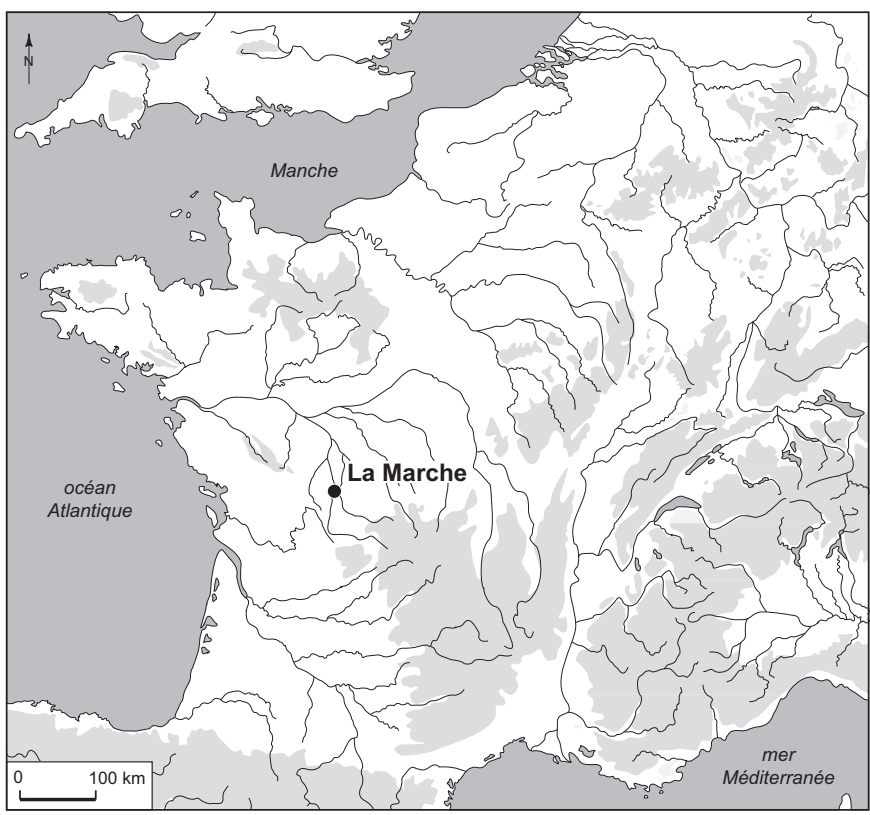

Fig. 1 - Localisation géographique du site de la Marche à Lussac-les-Châteaux (Vienne).

d'une recherche dans le domaine des représentations préhistoriques. Une partie des analyses a en effet été effectuée avec des moyens techniques nouveaux, que nous avons en partie expérimentés dans le cadre de notre thèse. Voilà pourquoi il nous a semblé important, au terme de celle-ci, d'effectuer également un bilan sur les atouts de ces moyens d'investigation pour la recherche préhistorique.

\section{PRÉSENTATION DU SITE}

La grotte de la Marche se trouve à Lussac-les-Châteaux (Vienne). Le village s'étend en longueur, parallèlement à un petit ruisseau dit «du petit Moulin ». Ce ruisseau est un affluent de la Vienne venant de l'est (sa longueur totale est d'environ $3 \mathrm{~km}$ ). C'est dans le vallon de celui-ci, à environ 1,5 km de son affluence avec la Vienne, que se présente un grand abri-sous-roche, la Marche (fig. 1). Ce gisement se trouve dans une région de grand intérêt pour la recherche.

Depuis juin 1990, on connaît, à quelques mètres au-dessus de l'abri de la Marche, le réseau Guy-Martin, une petite grotte ornée qui correspond au prolongement d'une petite cavité s'ouvrant vers le sud-ouest au sommet des falaises. Cette grotte abrite un ensemble majeur de gravures: celui-ci se trouve dans une des trois salles distinguées dans le réseau karstique, à environ $15 \mathrm{~m}$ de l'entrée. Les figura- 
tions comportent des animaux (mammouth et cheval) ainsi qu'une représentation humaine: un nouveau-né associé à trois vulves gravées (Airvaux, 1998 et 2001). Une couche de vestiges osseux provenant du remplissage est datée d'environ 14200 ans B.P. (Orsay-3780 = $14240 \pm 85$ ), ce qui correspond au Magdalénien moyen. Cette date est presque identique à celle obtenue à la Marche.

Hormis cette petite cavité où l'on trouve de l'art pariétal, on connaît d'autres sites sous forme d'abri dans lesquels des vestiges du Magdalénien moyen ont été découverts.

La grotte des Fadets se situe à environ $500 \mathrm{~m}$ de la Marche et comporte, à côté de vestiges moustériens remaniés, des couches solutréennes et magdaléniennes. Elle est datée de 15300 B.P. environ (Gif: 15300 B.P. \pm 150 ) (Airvaux, 2001).

Le célèbre site du Roc-aux-Sorciers à Angles-sur-l'Anglin (Vienne), avec ses frises sculptées, gravées et peintes, se trouve quant à lui à environ $30 \mathrm{~km}$ au nord. Il fait partie des sites qui, d'un point de vue stylistique, peuvent être rapprochés des modes de représentation des gravures de la Marche, notamment en ce qui concerne les représentations humaines; ces dernières sont par ailleurs très particulières (Airvaux, 2001; Auzanne, Fuentes, 2003).

Nous reviendrons dans cet article sur certains de ces sites. Nous rappelons d'ores et déjà que la Marche garde un caractère très original et possède une place à part, notamment en ce qui concerne le nombre de supports gravés.

\section{TOPOGRAPHIE ET STRATIGRAPHIE}

La grotte de la Marche se présente comme un grand abri semi-circulaire de $20 \mathrm{~m}$ de largeur et d'une profondeur, assez importante, de $19 \mathrm{~m}$. Son ouverture est orientée plein sud. L'entrée se trouve à environ $7 \mathrm{~m}$ au-dessus du niveau actuel du ruisseau (fig. 2).

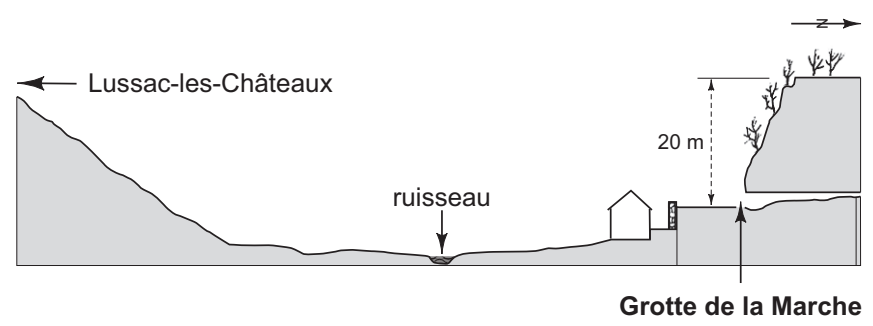

Fig. 2 - Profil de la vallée où se situe la grotte de la Marche (d'après Pales, Tassin de Saint-Péreuse, 1969).
La topographie du site a fortement changé par rapport à son aspect d'origine au Magdalénien comme cela a été montré par L. Pales. La partie de l'entrée a subi un remaniement particulièrement intense; elle a été aménagée bien avant la découverte du site et les premières fouilles. Des murets et un sol battu à l'intérieur datent certainement du XVIII $^{\mathrm{e}}$ siècle. Des plaquettes gravées magdaléniennes ont été trouvées dans ce muret (Lwoff, 1940; Pales, Tassin de Saint-Péreuse, 1969). Il est donc clair que la couche magdalénienne à plaquettes gravées fut en partie détruite lors de l'aménagement de la grotte qui eut lieu il y a plus de 200 ans. De même, on trouve aujourd'hui, devant l'entrée de l'abri, une terrasse artificielle qui forme un plateau. Afin de réaliser cette plate-forme, un mur a été construit parallèlement à la pente. Cet aménagement a changé de manière considérable la topographie de la zone située devant le porche. Nous n'avons en conséquence pas d'informations sur l'extension du site vers l'extérieur.

Le porche lui aussi a subi de nombreux remaniements naturels et artificiels. Autrefois largement ouverte, l'entrée se présente aujourd'hui divisée en deux portes aménagées. Les deux portes sont placées de part et d'autre de l'ouverture de la cavité, dans un mur contenant deux gros blocs d'effondrement et un comblement par des murs maçonnés. L'historique de la morphologie du porche que L. Pales a élaboré montre qu'au temps des Magdaléniens l'entrée était beaucoup plus large qu'aujourd'hui (Pales, Tassin de SaintPéreuse, 1969).

En raison des remaniements et des impacts des fouilles anciennes, la stratigraphie du site est difficile à établir (fig. 3). Lors de ses travaux de terrain, J. Airvaux a relevé un profil témoin qui a été conservé près de l'entrée (Airvaux, 2001). Le remplissage à cet endroit a une épaisseur de $1,50 \mathrm{~m}$. Il repose directement sur le substrat solide en calcaire et renferme quatre couches (Airvaux, 2001).

À la base se trouve la couche de vestiges préhistoriques dont l'épaisseur est de $10 \mathrm{~cm}$ à $30 \mathrm{~cm}$. Une datation au Carbone 14 l'attribue au Magdalénien moyen (Ly 2100: $14280 \pm 160$ B.P.). Elle se caractérise par une forte rubéfaction du sédiment dolomitique, apparemment provoquée, d'après les fouilleurs successifs (Pradel, 1958; Airvaux, 2001), par un apport d'hématite anthropique. À l'intérieur de la couche préhistorique s'intercalent de nombreuses lentilles sédimentaires stériles. La couche située au-dessus (3s) de la couche archéologique ne se distingue pas fortement du point de vue sédimentologique, puisqu'elle se caractérise, elle aussi, par un sédiment dolomitique. Cependant, elle ne contient aucun vestige archéologique 


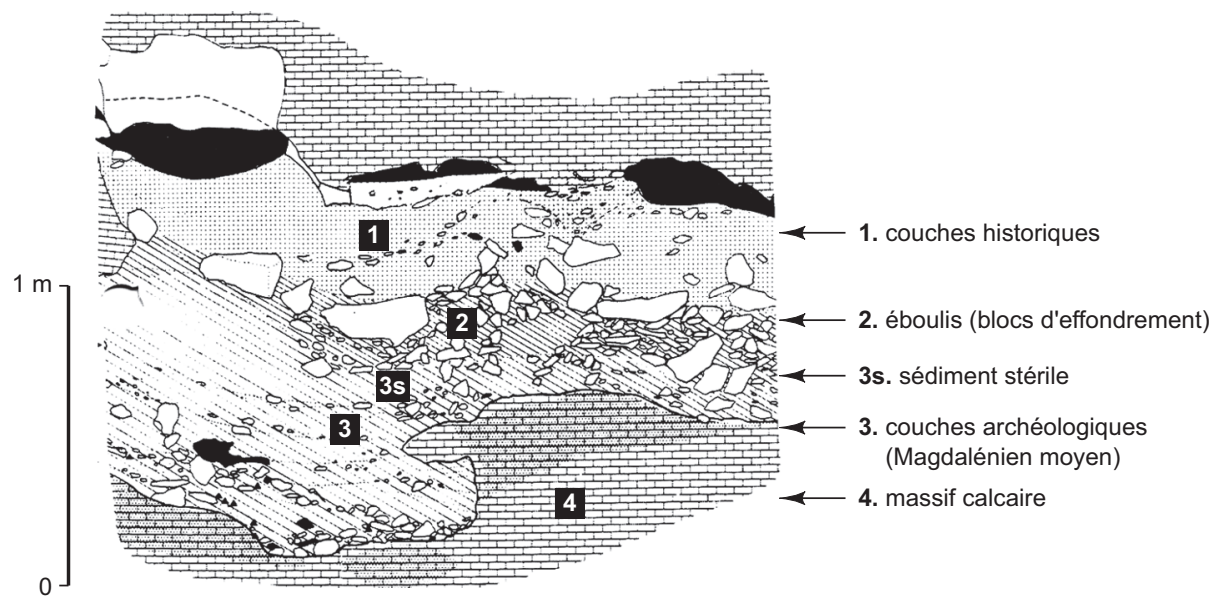

Fig. 3 - Relevé stratigraphique du remplissage de l'abri de la Marche. Coupe de référence (d'après Airvaux, 2001).

mis à part quelques petites esquilles (Airvaux, 2001). Les deux premières couches sont recouvertes par une strate d'éboulis de plaques calcaires. Au sommet de la coupe, une couche supérieure à sédiments dolomitiques sablonneux et contenant des vestiges historiques, tels que des tessons et des tuiles, complète la stratigraphie.

La stratigraphie décrite est donc une coupe de référence qui montre que la couche magdalénienne est en contact direct avec la roche de la falaise. Comprendre la topographie d'origine du site reste cependant difficile.

\section{PLAN DU SITE MAGDALÉNIEN}

Pour bien saisir comment ces informations ont été réunies et quelle est l'histoire du site, il convient de faire un détour sur les fouilles qui se sont succédé sur ce site.

L'intérieur de l'abri a en grande partie été fouillé dans les années 1930-1940 par S. Lwoff et L. Péricard. Les fouilleurs avaient déjà constaté le fort remaniement des sédiments dû aux aménagements historiques. Malgré cela, $\mathrm{au}$ fond de la grotte, des structures en place, notamment deux foyers, ont été découvertes. Les plaquettes gravées ont, elles, été trouvées partout dans le gisement et surtout vers la partie reculée. Les informations sur le plan de répartition des vestiges restent en revanche très rudimentaires.

Nous avons essayé de réunir les informations disponibles dans les publications anciennes pour reconstituer au mieux le plan du gisement: certains éléments nous ont permis de reconstituer des zones bien délimitées et plus ou moins riches en plaquettes (fig. 4). Ce travail sert ainsi notre objectif central, celui de mieux cerner le rôle des plaquettes dans le site. Mais pour cela, il faut également saisir les caractéristiques propres aux pierres gravées elles-mêmes.

\section{LA COMPLEXITÉ DES PIERRES GRAVÉES: PROBLÈMES SPÉCIFIQUES}

L'homme en tant qu'artisan, en tant qu'artiste ou encore en tant que consommateur, intervient de plusieurs manières sur la matière. Dans la plupart de ses activités, il entre en effet en interaction avec des matières premières (végétales, animales ou minérales) afin d'en faire usage. Dans ce processus, il les modifie plus ou moins fortement. Ceci est le cas pour les pierres gravées. Nous pouvons, à ce titre, distinguer deux groupes d'actions anthropiques sur les supports lithiques.

Les actions que nous appellerons « actions de premier ordre " réunissent tous les gestes et actions effectués dans l'intention de modifier la surface du support (en l'occurrence la pierre calcaire), dans le but de produire une représentation quelconque.

Le deuxième type d'actions que nous appellerons « actions de second ordre » regroupe des actions provoquant des modifications de surface qui prennent la forme de traces, témoins involontaires de travail par exemple. Elles ne relèvent donc pas d'une intention délibérée.

Sur les pierres gravées de la Marche, nous voyons que les actions primaires et secondaires peuvent se succéder à plusieurs reprises et ainsi former des superpositions. Une pièce peut de ce fait comporter plusieurs types de traces associés. L'ensemble de ces traces d'actions de premier et de 


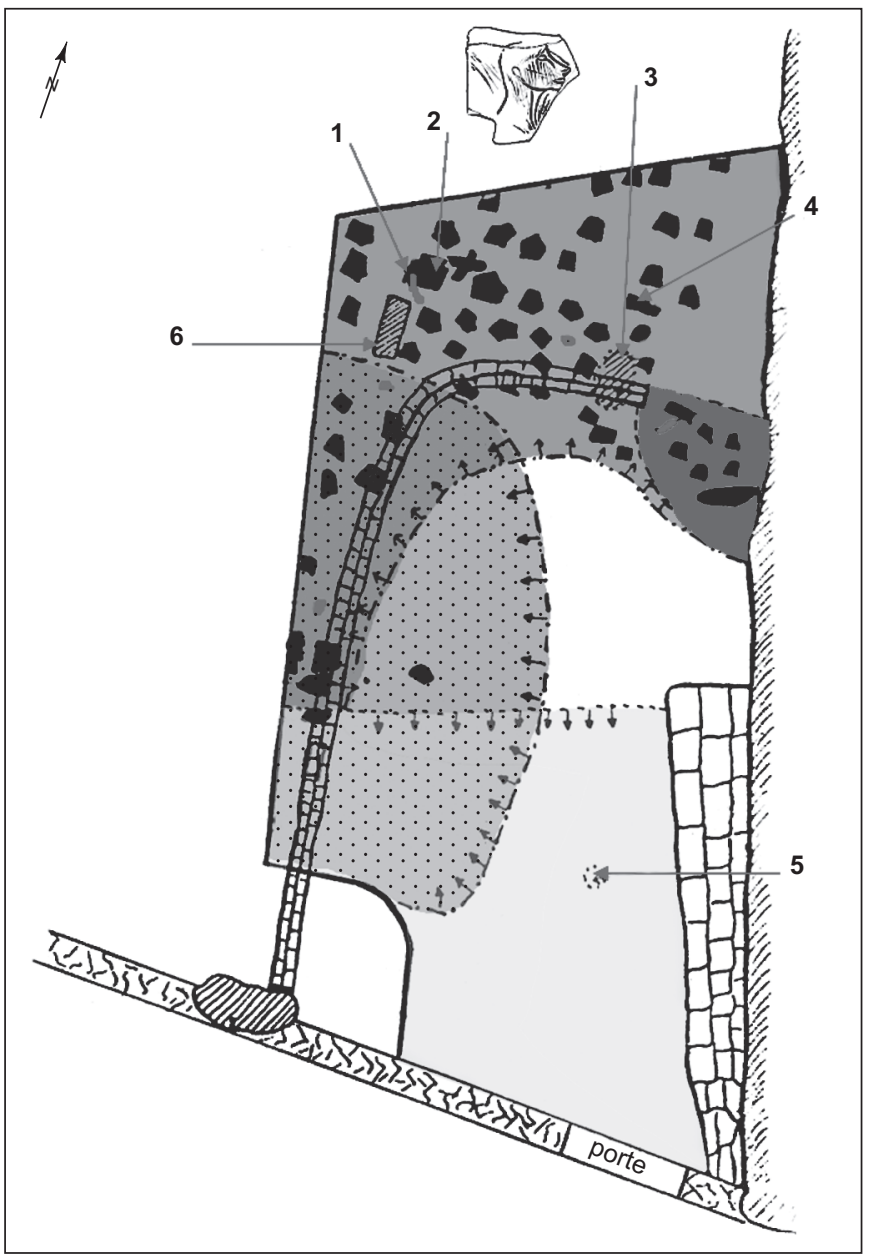

$\square$ dalles, parures, aiguilles
$\square$ plaquettes gravées
1. bâton percé en bois de renne
2. plaquette gravée avec tête humaine (profondément gravée)
3. foyer lenticulaire
4. pierre à patte de félin sculptée
5. cache à grandes lames
6. foyer en auge

Fig. 4 - Schéma de synthèse sur la répartition spatiale des vestiges dans le site. Données obtenues grâce aux publications des premiers fouilleurs de la Marche. Sans échelle (modifié d'après Lwoff, 1940, 1962a et b, 1965).

second ordre génère un palimpseste plus ou moins dégradé qui rend difficile sa lecture; on trouve rarement une seule figuration sur une surface gravée. Ceci constitue sans doute une des difficultés spécifiques aux supports mobiles lithiques gravés.

C'est à partir de ces considérations préalables que nous avons, dans le cadre de cette étude, élaboré nos objectifs généraux, objectifs qu'il convient maintenant de resituer.

\section{OBJECTIFS GLOBAUX}

Depuis le début des travaux intensifs sur les représentations de la Marche, les motifs variés et originaux ont conduit à des interprétations diverses des représentations. Or, les premières publications du matériel issu des travaux de J. Airvaux ont montré que, dans cet ensemble, se trouvent des représentations d'un très grand intérêt pour l'iconographie de la Marche et du Magdalénien moyen en général (Airvaux et al., 1991; Airvaux, 2001 et 2002). L'intégralité des pierres gravées inédites de la Marche nous semble ainsi être un sujet d'étude très probant.

Notre démarche comporte deux grands axes. Nous avons d'abord tenté de compléter le corpus des représentations, au moyen d'un travail de déchiffrement sur un certain nombre de pierres inédites. Mais nous avons eu comme objectif de pousser la démarche au-delà du simple déchiffrement des représentations. Notre but était de réaliser une analyse précise des pierres de la Marche choisies pour notre travail et d'aboutir à une interprétation détaillée de celles-ci. Pour ce faire, deux grands angles de vue ont globalement été retenus: il nous a semblé en effet indispensable de considérer ces objets de la même façon que d'autres vestiges laissés par les hommes préhistoriques, c'est-à-dire d'une part comme des témoins artistiques et d'autre part comme des témoins techniques et culturels du passé. Ainsi n’avons-nous pas uniquement considéré les plaquettes gravées comme des œuvres d'art (quand bien même nous pourrions utiliser ce terme pour les représentations préhistoriques), mais avant tout comme des objets archéologiques qui témoignent d'activités humaines quotidiennes ou bien épisodiques au sein d'un gisement magdalénien: notre étude a pour but de replacer les pierres de la Marche dans leur contexte archéologique.

Notre approche a donc comporté plusieurs démarches d'analyse complémentaires, qui ont servi à envisager les objets dans leur intégralité. À ce titre, il convient de présenter les principaux aspects des pierres gravées qui ont été retenus et analysés pour répondre à l'objectif global que nous venons d'évoquer.

Le cœur de notre étude concerne les gravures ellesmêmes, dans la lignée des recherches précédentes, mais en utilisant des moyens techniques qui n'étaient pas disponibles avant. On peut se demander, en effet, quel intérêt il y a à revenir sur ce thème déjà bien étudié. Plusieurs arguments qui nous ont servi de cadre pour cette étude peuvent être avancés.

Comme nous l'avons souligné, la recherche sur les pierres de la Marche se heurte toujours au problème du 
palimpseste. Celui-ci a pour l'instant empêché l'exhaustivité des analyses et des interprétations envisageables. Voilà pourquoi le travail n'est pas terminé.

De même, mise à part l'étude stylistique des représentations, nos observations et nos analyses ont particulièrement porté sur la morphologie des traits ainsi que sur la reconstitution des gestes du graveur (D’Errico, 1988; Fritz, 1999; Marshack, 1970). Nous nous sommes donc particulièrement penchés sur les techniques de gravure et sur le sens qu'on peut leur attribuer.

Enfin, dans les enchevêtrements de traits que nous avons déjà signalés, on trouve des représentations d'animaux, parfois d'humains mais, souvent aussi, de nombreux traits n'appartenant pas aux figurations. En ce qui concerne ces derniers, il s'agit la plupart du temps de gravures intentionnelles mais dont la signification nous échappe. Nous nous sommes par conséquent interrogés sur l'origine et la raison de tous ces traits indéterminés et sur leur rôle au sein de l'iconographie de la Marche. C'est pour cette raison que nous n'avons pas restreint nos relevés aux pièces les plus remarquables, portant des figurations intelligibles, et que nous les avons étendus aux pièces qui ne comportaient pas de représentations identifiables.

Le deuxième aspect de notre démarche concerne les supports eux-mêmes mais aussi les traces relevant de ce que nous avons appelé les " actions de second ordre " (voir supra). Plusieurs éléments expliquent que l'on se soit attaché à ces éléments.

Nous avons d'abord étudié précisément la nature pétrographique des supports. Celle-ci nous permet en effet d'interroger leur provenance géographique mais aussi de déterminer leurs propriétés mécaniques. Ceci a par ailleurs été précieux pour analyser les liens qui peuvent exister entre le support et la gravure.

Toutes les autres traces et particularités morphologiques, qui ne sont pas des gravures mais se trouvent sur les supports, constituent aussi des éléments très intéressants à étudier. En effet, les pierres de la Marche, comme tout objet archéologique, ont circulé pendant un temps plus ou moins long dans le gisement. Ainsi, souvent gravées à plusieurs reprises, usées, fracturées et donc complexes, les plaquettes et dalles gravées de la Marche portent un ensemble de marques et traces diverses qui témoignent de leur « historique ». Nous entendons par « historique » des supports gravés, la suite des événements qui s'intègre entre le choix et le transport des supports bruts dans le site et leur abandon, et ce, en passant par la production des gravures et leur éventuelle réutilisation.
Comme nous nous sommes donné pour objectif l'analyse détaillée de tous les éléments que nous venons de présenter, et notamment leur caractérisation quantitative et qualitative afin de les comparer et de les interpréter, nous avons eu recours aux moyens techniques les plus avancés que nous ayons à notre disposition.

À ce titre, les sciences naturelles proposent aujourd'hui une quantité importante de techniques d'analyse, qui sont empruntées par les chercheurs en archéologie et préhistoire. On peut, par exemple, rappeler les grandes avancées que l'on doit aux applications des sciences de la vie et de la terre à des questions archéologiques, comme les datations absolues et l'analyse morphologique des vestiges archéologiques. Dans ce domaine, on utilise désormais des techniques optiques d'observation, des études physico-chimiques de matériaux et de la tracéologie, par le biais notamment du microscope électronique à balayage (D'Errico, EspinetMoucadel, 1986; Fritz, 1999; Airvaux, 2002). Tous les domaines de la Préhistoire sont concernés par ces progrès méthodologiques et techniques des moyens d'investigation. Ceux-ci nous permettent d'étudier les objets provenant de fouilles actuelles mais donnent également la possibilité de reprendre des collections anciennes et de compléter des données issues de travaux antérieurs.

Nous avons ainsi, pour notre étude des pierres de la Marche, réuni un ensemble de techniques d'analyse qui permettent une approche très précise, en fonction de nos questionnements, des différents aspects des supports gravés. Aux descriptions et aux relevés des supports et des représentations ont donc été ajoutés d'autres moyens d'investigation afin d'appréhender les domaines stylistique, technologique et fonctionnel. Une telle étude est transdisciplinaire et a nécessité l'utilisation de différentes techniques: celles-ci ont été empruntées au domaine de l'optique (photographie, projection, microscopie) et à ceux de la physique (microscope électronique à balayage, microrugosimètre) et de la chimie (spectrométrie).

Pour achever cette présentation de la problématique qui a guidé notre travail, nous tenons à mentionner un point important. Nous avons, dans le cadre de cette étude, tenté de mettre en lumière cet ensemble très riche d'objets, qui appartient par ailleurs à un site majeur pour ce qui est des représentations magdaléniennes. Mais nous avons aussi voulu proposer un protocole d'étude qui permet de répondre au mieux aux questions de l'archéologue tout en assurant la conservation des objets.

Voilà pourquoi, avant d'entamer la présentation des analyses générales, il nous semble judicieux de passer en 
revue les moyens d'études mis en œuvre dans le cadre de notre travail. Leurs spécificités pourront ainsi être mieux saisies et remises dans le contexte de leur emploi.

\section{DÉMARCHES ET TECHNIQUES}

\section{MÉTHODE UTILISÉE POUR L'ÉTUDE DU CORPUS}

L'intégralité des objets de la Marche, soit près de 3000 pièces, se répartit dans différentes collections, sachant que 217 pièces remontées ont déjà été étudiées par L. Pales. Nous avons, dans le cadre de ce travail, effectué un choix. Toutes nos statistiques et études majeures se basent ainsi sur les plaquettes que nous avons nous-mêmes inventoriées au Service régional de l'archéologie de Poitiers et qui se composent de 1409 pièces exactement. Celles-ci font partie des fonds du musée Sainte-Croix à Poitiers. À cet ensemble s'ajoutent, pour l'étude générale et approfondie, cinq pierres issues de collections anciennes. Elles ont particulièrement retenu notre attention parce qu'elles possèdent des figurations animales et humaines intéressantes par leur qualité et/ou leur densité. En effet, une grande partie des pierres du corpus inédit portent des traces qui n'appartiennent pas à des figurations concrètes. Allier les deux nous permettait donc d'étoffer la variété iconographique, afin de réaliser l'étude la plus complète possible.

L'origine même des pierres ajoutées à notre corpus est variée. Une d'entre elles provient de la collection Péricard conservée au musée Sainte-Croix à Poitiers: LP 22 (pl. 18 à pl. 21). De cet ensemble de 422 pierres, 93 ont déjà été étudiées par L. Pales. Celle que nous avons retenue était elle-même comprise dans cette étude.

Quatre autres sont, quant à elles, conservées au musée de l'Homme: MH 50-7-42, MH 50-7-275, MH 50-7-276 et MH 50-7-300 (pl. 1 à pl. 17) .

L'étude de ces pièces a, dans un premier temps et pour tous les spécimens du corpus, nécessité l'établissement d'une base de données selon des paramètres adaptés à notre problématique. Sur l'inventaire résultant, un échantillonnage des pierres les plus représentatives de chaque paramètre répertorié a été établi. Celles-ci (49) ont ensuite fait l'objet d'une analyse plus approfondie, souvent par relevé manuel puis par des moyens d'investigation plus précis tels que le microscope électronique à balayage (MEB) ou le microrugosimètre.

Une présentation de ces étapes d'étude selon l'ordre chronologique de leur application semble nécessaire.

\section{INVENTAIRE ET ÉCHANTILLONNAGE}

Il nous a donc fallu d'abord procéder au tri des pierres. Pour chacun des objets, un certain nombre de paramètres quantitatifs (dimensions, nombre de figurations, etc.) et qualitatifs a été retenu. Ces derniers concernent aussi bien des aspects archéologiques comme l'art gravé, les résidus de pigments et les témoins d'utilisation que des caractéristiques pétrographiques des supports comme la texture, le litage ou la coloration. Ces informations ont été retenues sur une fiche descriptive par la suite numérisée, afin de pouvoir être exploitable par le biais d'analyses statistiques (fig. 5). In fine, chaque pièce a été caractérisée par 19 catégories d'attributs. Chaque élément a été reporté sur la fiche sous forme de code.

Lors de la réalisation de ce catalogue, une observation soignée à l'échelle macroscopique et à la loupe binoculaire a été réalisée et des commentaires supplémentaires ont été notés.

C'est sur la base des phénomènes observés sur les pierres gravées que nous avons procédé à un échantillonnage, afin d'entreprendre des études plus poussées sur les supports. Au-delà de l'analyse statistique, certaines pièces (56) ont ainsi été retenues parmi l'ensemble pour une étude plus précise par des moyens d'investigation adaptés.

\section{DOCUMENTATION PHOTOGRAPHIQUE}

La première étape de l'étude systématique est le relevé photographique. La photographie des objets est d'une importance double pour le travail scientifique sur les pierres gravées. La photo sert d'abord à la documentation graphique de l'objet. Mais, pour l'étude même, elle constitue également un outil de travail très important. Lors des prises de vue, l'éclairage de la pièce et l'observation des gravures ainsi que des aspects de surface sous différents angles sont indispensables. Comme toujours, dans le travail autour de ce type de supports, se pose le problème de la lecture des gravures, rendue difficile à cause de la haute densité des traces ou de la finesse des gravures, ou encore à cause de l'état de conservation des pierres. En effet, l'altération des supports a parfois entraîné une coloration irrégulière de la surface ou provoqué une dégradation des traits incisés jusqu'à effacement complet.

De ce fait, l'approche de la pièce se précise de plus en plus lors de cette étape. Se posent alors des questions sur la nature et l'état de conservation des tracés et sur l'existence de formes révélant de nouvelles figurations. D'autres 


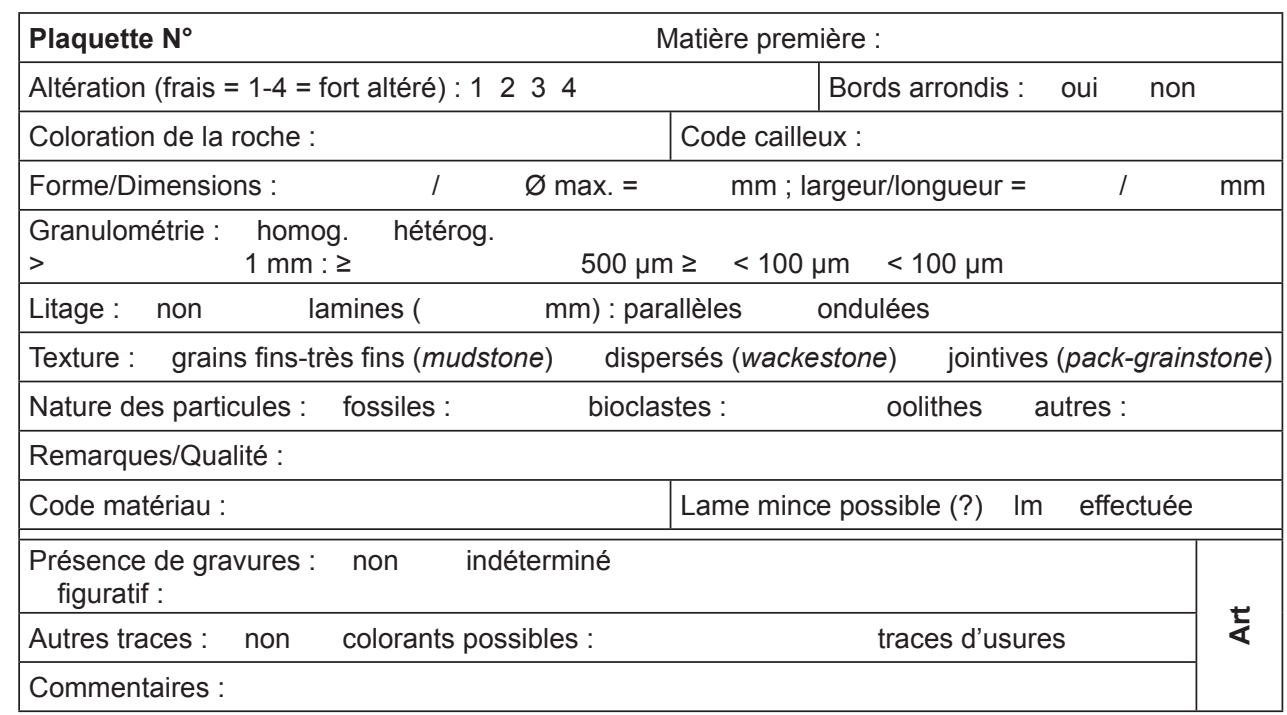

Fig. 5 - Fiche descriptive pour linventaire des pierres inédites de la Marche.

questions interviennent aussi, souvent inspirées par l'état des bords et la relation des gravures avec ceux qui sont fracturés. Elles portent sur l'historique du support de sa production jusqu'à son dépôt. Tout cela apparaît déjà lors de l'observation du photographe et peut être approfondi par les études suivantes (voir infra). Il est donc indispensable que la personne qui étudie les supports réalise les photographies ou du moins soit présente lors de la prise de vue. Ceci est d'autant plus important quand la photographie sert de support pour un relevé.

La technique de prise de vue peut être différente selon les pièces. Nos photographies ont été prises soit avec un appareil argentique et des tirages en diapositive, soit avec un appareil numérique. Pour le traitement numérisé ultérieur des images, il s'est avéré que les prises de vue numériques étaient plus pratiques que les clichés sur diapositive ou tirage papier mais, en ce qui concerne la qualité d'image, nous n'avons pu distinguer une des deux techniques comme étant meilleure que l'autre.

En règle générale, les photographies doivent être prises avec des éclairages différents afin de faire apparaître tous les traits sur les pièces. La source lumineuse peut être soit la lumière du jour si elle est assez rasante (matin ou soir) soit une source artificielle assez puissante. Le faisceau lumineux doit être le plus parallèle possible et être positionné assez loin du support, afin de permettre une bonne diffusion de la lumière et éviter ainsi les surexpositions dans les endroits proches de la source lumineuse. Une seule prise de vue ne peut quasiment jamais représenter l'intégralité des traces sur une pièce.

Les prises de vue que nous avons effectuées nous ont fourni des supports très adaptés à plusieurs étapes de l'étude, comme par exemple le relevé manuel. En effet, en ce qui concerne certains supports, le calque sur photographie s'est révélé être un moyen très précis pour le déchiffrement des gravures. Mais il convient, avant toute chose, de préciser le rôle et l'importance que l'on accorde au relevé lui-même.

\section{LE RELEVÉ (RÔLE GÉNÉRAL ET TECHNIQUES)}

L'étape du relevé est un passage indispensable dans l'analyse de représentations préhistoriques. Son importance est multiple.

Il constitue d'abord, bien entendu, une reproduction plus lisible des gravures et ainsi une aide à la lecture. Il est de plus possible de réaliser des relevés sélectifs en cas de superposition des figurations et des traces. La fonction essentielle du relevé est cependant son caractère analytique qui s'impose automatiquement. Le questionnement spécifique à une pièce, qui concerne par exemple les figurations et les types de traits, s'installe lors de ce travail. Ceci a été constaté depuis plusieurs années par différents chercheurs, aussi bien dans le domaine des représentations dans les grottes que pour ce qui est des supports mobiliers (Vialou, 1982; Delluc, Delluc, 1984; Lorblanchet, 1995). Comme le 
relevé dépend largement de la problématique et de l'approche méthodologique adoptées par chaque chercheur, il en résulte des modes opératoires et des visions diversifiées de ce relevé qu'il est bon d'évoquer brièvement pour resituer notre démarche: le rôle que nous lui avons accordé sera par là même replacé dans le contexte plus général du recours à ce procédé au sein des recherches préhistoriques.

Les travaux de M. Lorblanchet nous montrent à quel point un lien existe entre le support et les représentations. C'est cette conception qui se manifeste dans ses relevés. Ceux-ci sont en conséquence plus qu'un travail de reproduction; ils deviennent un moyen de mise en relation des représentations et des supports (parois des abris et cavernes). Ils sont ainsi le résultat d'une interprétation.

D. Vialou a également une expérience multiple du relevé archéologique, aussi bien dans le cadre de l'art mobilier (la Marche) que dans celui des représentations pariétales (Lascaux, Aldène, Niaux, etc.). Il considère le relevé comme un moyen d'investigation important pour l'étude approfondie des représentations préhistoriques (Vialou, 1976 et 1982). Le relevé est bien sûr un moyen d'aide à la lecture des photographies et constitue une illustration lisible pour les publications; mais il est surtout à la fois support et résultat d'analyse. C'est dans le relevé final d'une représentation que se manifeste la synthèse d'une étude précise.

Le travail de B. et G. Delluc se place également dans cette démarche d'observation technologique (Delluc, Delluc, 1984). Ils utilisent depuis plusieurs dizaines d'années le relevé des représentations préhistoriques de différentes manières. Pour améliorer la lecture et la compréhension du relevé, ils ont ainsi élaboré des figurés standardisés. Mais plus encore, ils ont élaboré un vocabulaire technique et une légende de lecture qui leur permettent de faire apparaître des aspects technologiques des traces anthropiques (gravures, piquetages, raclages, etc.) et animales (griffures, griffades, etc.) ainsi que des informations sur les supports (fissures, altérations).

Sur le site de Gönnersdorf, en Allemagne, G. Bosinski et $\mathrm{G}$. Fischer ont été placés face au problème du relevé de plaquettes et plaques gravées couvertes d'un dense entrecroisement de traits fins dans lequel se trouvaient des figures d'animaux et de femmes schématisées.

Aussi les chercheurs ont-ils élaboré leur propre conception du relevé, adaptée à la problématique spécifique que posent les plaquettes de schiste de ce site et les traces qui s'y trouvent (Bosinski, Fischer, 1974). En effet, la plupart des relevés ont été effectués par calque direct sous binoculaire, avec une lumière rasante. De même, pour faire apparaître les figures cachées dans les enchevêtrements de traits, des relevés sélectifs qui facilitent la lecture ont été ajoutés.

Nous voyons donc, dans ce bref aperçu, que les techniques de relevé employées par des personnes différentes peuvent être très diverses. Elles varient également en fonction du support et des problèmes d'étude spécifiques à chaque site et à chaque démarche.

Certains des buts communs recherchés sont cependant la lecture objective des traces sur les pierres et la reproduction la plus fidèle possible des figurations.

Il n'en reste pas moins que le relevé manuel garde toujours une part de subjectivité et qu'il reste incomplet, notamment lorsqu'il s'agit de supports très complexes. Il est par exemple très difficile de relever des traits indéterminés que l'on n'arrive pas à attribuer à une figuration précise. En revanche, une fois une figuration identifiée, il est difficile de l'ignorer pour concentrer le regard sur d'autres tracés identifiés.

Bien que le relevé soit un exercice difficile, il reste cependant une étape primordiale pour l'étude et la compréhension du support étudié. C'est par conséquent une étape que l'on ne peut omettre mais sur laquelle il convient, en tant que chercheur, de réfléchir.

C'est pour cette raison que nous avons pris un soin tout particulier au développement de ce stade d'étude décisif dans l'analyse détaillée des pierres: celui-ci a en effet guidé la suite de notre travail. Nous avons utilisé différentes techniques de relevé, en fonction des pierres, mais aussi du matériel disponible.

\section{Les relevés d'après photographies}

Un des moyens utilisés pour le relevé graphique d'une pièce gravée est donc le calque sur photographie. Il présente plusieurs avantages, surtout par rapport au calque direct. En premier lieu, il s'agit bien sûr d'une technique qui évite tout contact direct avec le support. De plus, le tirage photographique supprime l'irrégularité de la surface et permet un meilleur contrôle du parcours du crayon lors du relevé. Enfin, pour les petites pièces notamment, il est très utile d'agrandir le tirage par rapport à l'original: la visibilité et le relevé des divers contours et gravures en sont ainsi plus aisés.

Pour réaliser un calque sur photographie nous avons procédé de la manière suivante: les prises de vue numériques ou numérisées ont été montées sous forme de mosaïque dans un logiciel adapté. Chacun des éléments a été choisi pour sa qualité de représentation des tracés. Le but était d'afficher une image qui puisse reproduire 
la totalité ou au moins le maximum des traits gravés sur les pièces. Une fois cette image générée, un tirage papier a été réalisé à l'échelle souhaitée et celui-ci a ensuite été décalqué sur un transparent ou un dépoli pour obtenir le relevé manuel de la pièce.

Afin de s'assurer que la totalité des tracés pouvait être représentée, plusieurs supports photographiques ont été utilisés pour le calque.

Lors de l'élaboration d'un relevé, le recours à l'original pour comparaison et vérification est nécessaire afin d'effectuer d'éventuelles corrections.

Tout intéressant que soit ce mode de relevé, ce n'est pas le seul que nous avons utilisé.

\section{Les relevés par rétroprojection}

La technique du relevé par rétroprojection, avec une chambre photographique de grandes dimensions disposée au-dessus de l'objet, est un outil de travail très adapté au relevé de supports de taille réduite, ne dépassant pas 10-15 cm (Airvaux, 1984 et 2002; Airvaux et al., 1991). Néanmoins, des pièces plus grandes peuvent faire l'objet de relevés. Dans ce cas, le relevé doit être réalisé en plusieurs fois, chaque calque correspondant à une partie de l'objet. Leur montage permet ensuite d'obtenir le relevé complet.

On peut noter plusieurs avantages à l'application de la projection directe dans la réalisation du relevé.

L'image de la surface gravée est d'abord arrêtée sur un dépoli (calque). Une surface en relief est donc projetée sur une surface plane, ce qui rend le dessin plus aisé. Comme pour la photographie, l'éclairage se constitue de sources lumineuses mobiles. L'avantage ici est de pouvoir changer le type de sources lumineuses et l'angle d'éclairage lors de la réalisation du relevé autant de fois que nécessaire jusqu'à l'obtention du relevé exhaustif.

Nous avons utilisé cette technique pour la plupart des petites plaquettes conservées à Poitiers car son application nécessite l'installation d'une chambre photographique adaptée dans les locaux. Pour les pièces du musée de l'Homme, nous avons eu recours au relevé sur photographie.

Il convient enfin de noter que, quel que soit le type de relevé effectué, l'emploi de la loupe binoculaire peut être parfois intéressant.

\section{L'emploi de la loupe binoculaire}

La loupe binoculaire que nous avons utilisée permet des agrandissements (jusqu'à x 50). Lors des travaux décrits jusqu'ici, ce matériel nous a permis de faire des observations rapides de certaines zones spécifiques de la surface. Par conséquent nous nous en sommes servi pour vérifier certains détails visibles sur celle-ci (gravures, aspérités, traces d'utilisation et de fracturation). Lors de relevés nous avons également eu recours à ces observations pour répondre à certaines questions, sur la morphologie d'un trait par exemple. Mais nous avons aussi et essentiellement utilisé cet appareil pour rechercher des résidus de pigments et pour leur prélèvement.

Parfois, l'observation des supports à la loupe binoculaire a révélé des traces modernes, telles que des résidus de matière utilisée pour les moulages ou encore des tracés de graphite effectués pour faire ressortir les traits gravés.

Cependant, dans l'observation des gravures, se pose le problème de la profondeur de champ ainsi que de l'éclairage qui rendent souvent difficiles la distinction des traits et l'étude de leur morphologie. Pour l'étape de l'analyse plus approfondie, le MEB et le microrugosimètre s'avèrent ainsi être des moyens d'investigation très adaptés.

\section{LES MOYENS D’INVESTIGATION}

\section{LE MICROSCOPE ÉLEGTRONIQUE À BALAYAGE (MEB)}

Nous avons travaillé, au sein du Centre de recherche et de restauration des musées de France, avec un microscope électronique à balayage de type PHILIPS XL Series XL 30CP. Ce dispositif est équipé de détecteurs différents qui permettent de générer des images fortement grossies. L'observation au MEB est en effet utilisée depuis un certain temps dans le domaine de la recherche sur les représentations préhistoriques, notamment sur les gravures. Elle livre des résultats intéressants pour la compréhension des œuvres (D'Errico, Espinet-Moucadel, 1986, 1993 et 1994; Fritz et al., 1993; Fritz, 1999). Nous avons cependant choisi, dans le cadre de cette étude, d'utiliser cet appareil exclusivement pour l'analyse des pigments, car nous avons renoncé au moulage et à la métallisation des œuvres.

Trois détecteurs du microscope ont été utilisés pour nos études.

Le détecteur des électrons secondaires (SE), d'abord, est monté à un certain angle de la trajectoire du canon à électrons et crée des images qui mettent en évidence la morphologie de la surface avec une très bonne résolution. Ainsi est-il possible d'étudier des formes et des types d'agencement des matières composées. Nous avons par exemple pu 
travailler sur la forme des minéraux formant les pigments ainsi que sur leur disposition.

Le détecteur des électrons rétrodiffusés (BSE) est, quant à lui, placé différemment par rapport à l'échantillon et ne donne pas une bonne idée du relief; il crée cependant une image en " contraste de phase ». Le principe est le suivant: le nombre d'électrons rétrodiffusés est variable et spécifique en fonction du nombre atomique de chaque élément; cette différence se matérialise dans l'image générée sous forme d'un dégradé de niveaux de gris allant du blanc au noir. Les éléments chimiques possédant un numéro atomique élevé (charge positive du noyau plus grand) produisent davantage d'électrons rétrodiffusés que ceux ayant un numéro atomique faible. Les zones de l'échantillon avec numéro atomique élevé sont donc plus blanches que celles ayant un numéro atomique faible. On appelle ce phénomène " contraste de phase ». Cette fonction est intéressante pour localiser les éléments sur la surface de l'échantillon. Dans notre cas, il a ainsi été possible d'identifier précisément certains éléments chimiques sur la surface.

S'ajoute enfin à l'étude des images une spectrométrie qui nous renseigne sur la nature des éléments chimiques dans l'échantillon. Un détecteur de rayons X (EDS) permet de fournir des informations pour analyser la chimie de la surface observée ou de certains éléments précis sur cette surface. En effet, le signal transmis par le détecteur fournit un spectre de pics d'amplitude. Celui-ci est représentatif des éléments chimiques qui se trouvent sous le faisceau. Il est de même possible d'étudier toute la fenêtre de microscopie ou bien des points précis dans l'image.

Si le MEB nous a donc été d'une aide précieuse pour approfondir certains aspects de notre étude, le microrugosimètre permet d'effectuer d'autres types d'analyse, notamment sur les gravures elles-mêmes et sur les supports, qui sont tout à fait intéressants pour notre travail.

\section{LA MICROTOPOGRAPHIE CONFOCALE À «CHROMATISAME AXIAL »}

Pour une grande partie de nos analyses concernant les gravures, les traces d'utilisation et d'usures ainsi que pour l'étude des microsurfaces des matières premières, nous avons utilisé la microtopographie.

Pour cela nous nous sommes servis d'une station de saisie microtopographique confocale à champ étendu. Ce relevé permet en effet de réaliser des images tridimensionnelles à très haute résolution (jusqu'à moins de 200 nm).
Le relevé microtopographique de la station de micromesure (STIL-CHR 150) est basé sur un balayage pas par pas d'une surface donnée avec un crayon optique. Lors de cette opération sont enregistrées les coordonnées spatiales en X, $\mathrm{Y}$ et $\mathrm{Z}$ de chaque point inclus dans le pas du balayage. La saisie fonctionne sans aucun contact avec la surface et est absolument non destructive.

\section{COMPOSITION ET FONCTIONNEMENT DE LA STATION DE MICROMESURE}

L'appareil est composé d'un crayon optique alimenté par une source de lumière blanche et monté sur une table de translation. L'émetteur de lumière comprend également un spectromètre et le tout est contrôlé à partir d'un ordinateur (fig. 6).

La configuration du crayon optique est telle que la lumière envoyée est diffractée sur un axe (chromatisme axial) par un objectif, selon des longueurs d'ondes colorimétriques allant du bleu au rouge, chaque couleur correspondant à un point. Le point qui se trouve exactement sur la surface envoie un signal qui est ensuite analysé et converti en valeur métrique $(Z)$. Celle-ci représente la distance entre la sortie du crayon optique et un point précis sur la surface: la combinaison de toutes les valeurs $\mathrm{Z}$ permet ainsi de mesurer les différentes hauteurs du support. La précision de la mesure de hauteur est de $0,156 \mu$, ce qui correspond à 0,00016 mm environ.

À l'enregistrement de la valeur Z un déplacement latéral en X, généré par la table de translation, est enregistré et attribué au $\mathrm{Z}$ correspondant. La finesse de la mesure $\mathrm{X}$ dépend du pas de mesure qui peut être fixé avant la saisie. Le chercheur a le choix entre des pas de $0,2 \mathrm{~mm}, 200 \mu$ ou $0,156 \mu$ comme en $\mathrm{Z}$. Le balayage latéral en X doublé par la mesure correspondante en $\mathrm{Z}$ nous livre un profil de la surface (fig. 7).

Or, les profils sont multipliés selon l'axe Y. Ainsi l'image de synthèse microtopographique se constitue-t-elle. La surface gravée correspond à un paysage avec des vallées et des sommets. Une fois les données enregistrées, elles peuvent être traitées par un logiciel de visualisation et d'analyse 3D adapté.

\section{Traitement des données}

Le traitement des données de base se fait à partir d'un logiciel adapté. Après une optimisation des données 3D (seuillage, rebouchage de points non mesurés, visualisa- 


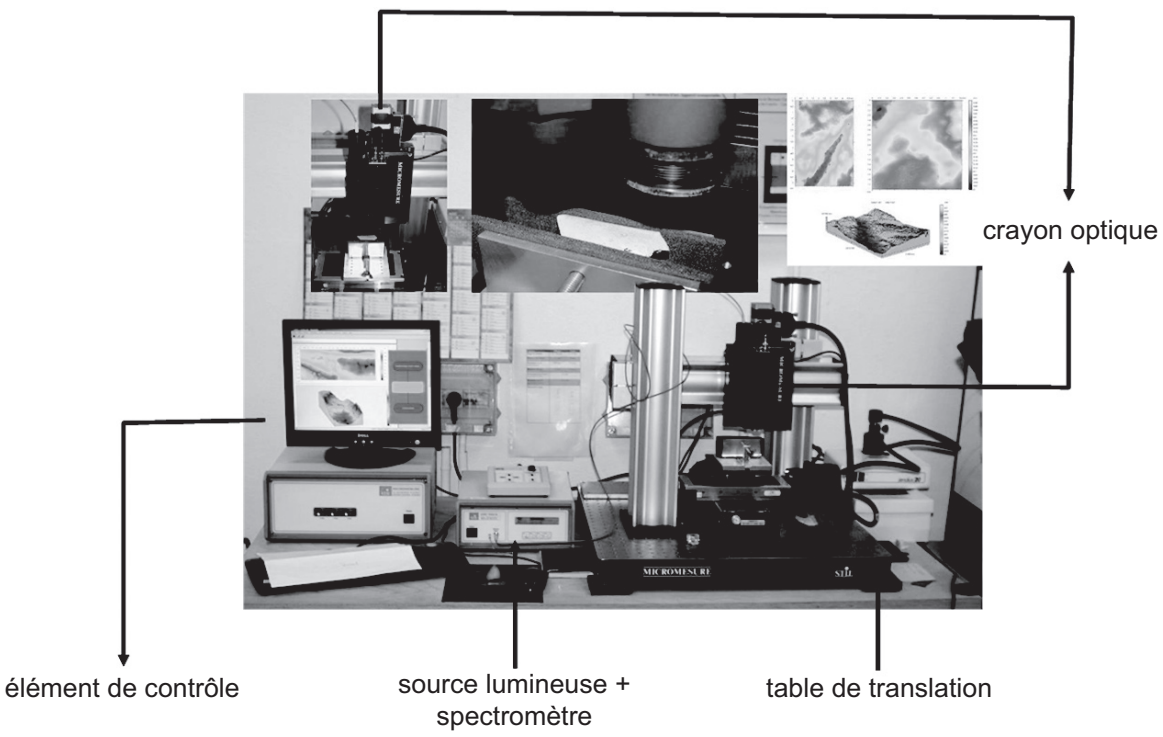

Fig. 6 - Photographie et schéma de la station de microtopographie (cliché : N. Mélard).

\begin{tabular}{|l|c|c|}
\hline & $\mathbf{3 0 0} \boldsymbol{\mu m}$ & $\mathbf{3} \mathbf{~ m m}$ \\
\hline Distance de travail $(\mathrm{mm})$ & 5,68 & 26,9 \\
\hline Résolution Z $(\mathrm{nm})$ & 10 & 100 \\
\hline Diamètre du faisceau $(\mu \mathrm{m})$ & 7,4 & 25 \\
\hline Résolution latérale $(\mu \mathrm{m})$ & 3,7 & 12,5 \\
\hline $\begin{array}{l}\text { Pas de saisies en } \mathrm{x} \text { et } \mathrm{x}(\boldsymbol{\mu m}) \\
\text { choisis pour l'étude }\end{array}$ & 0,469 & $5-10-15-20-200$ \\
\hline
\end{tabular}

Fig. 7 - Tableau synthétique des crayons optiques utilisés et des pas de saisies employés dans notre analyse.

tion), il est possible de reconstituer les surfaces gravées et de visualiser les gravures. On peut également tourner la lumière virtuellement pour examiner celles-ci.

L'utilité de ces outils d'analyse se dévoilera lors de la présentation des résultats de notre étude.

\section{Configuration et paramétrage}

Pour l'étude des pierres de la Marche, nous avons appliqué des protocoles de mesure adaptés aux différentes questions posées.

Globalement, nous pouvons distinguer deux types d'échelle. La première échelle concerne une étude plutôt qualitative des surfaces et gravures. La deuxième est plutôt ciblée sur la caractérisation quantitative de microsurfaces. Les deux approches ont été réalisées avec des protocoles et des paramétrages d'étude prédéfinis afin de pouvoir comparer les résultats.

\section{Étude qualitative et mesures de forme}

L'étude des surfaces sur lesquelles se trouvent les gravures présente deux contraintes majeures en ce qui concerne l'analyse microtopographique utilisée dans notre approche («le chromatisme axial»).

Normalement, il est nécessaire de relever des surfaces assez grandes pour capturer un nombre conséquent de gravures. Ceci est particulièrement important lorsque nous nous intéressons au déchiffrement des réseaux de traits. Ici se pose le problème de la profondeur de champ $(3000 \mu \mathrm{m})$. Tout écart hors de ce champ ne peut être mesuré en une seule fois. Par conséquent, une telle analyse requiert plusieurs relevés sur la surface. Pour le relevé de surfaces étendues s'ajoute la question de la résolution, c'est-à-dire du paramétrage des pas de mesure en X et Y. Nous avons choisi une configuration qui permet à la fois une précision assez élevée pour étudier la morphologie des traits et assez large pour éviter d'alourdir les fichiers à traiter et surtout de rallonger le temps de mesure.

Pour le déchiffrement, notre résolution en $\mathrm{X} / \mathrm{Y}$ a été de $30 \mu \mathrm{m}$ ou $20 \mu \mathrm{m}$; pour les études sur la morphologie ou l'état de conservation de surfaces, de traits nous avons effectué des mesures de $5 \mu \mathrm{m}$ ou $10 \mu \mathrm{m}$. Une fois les données enregistrées, celles-ci peuvent être traitées grâce au logiciel adapté (dans notre cas il s'agit du logiciel Mountainsmap/ DigitalSurf).

Après un seuillage des données et un rebouchage des points non mesurés, les images se prêtent très bien aux diverses visualisations. Celles-ci peuvent être réalisées en 
fausses couleurs (échelle de couleurs attribuée aux différentes hauteurs) ou en « simulation photo » (couleur uniforme de la surface). Cette dernière fonction permet de simuler une lumière qui provoquera un jeu d'ombres sur la surface de l'objet en fonction de la morphologie de celle-ci.

Les images issues des donnés 3D peuvent faire l'objet de mesures diverses. Tout d'abord, il est possible de mesurer des distances exactes sur la surface (dimensions de tracés, largeur). Chaque point étant codifié en coordonnées tridimensionnelles il est également possible de visualiser les surfaces dans des schémas en bloc. Les données permettent de la même façon d'extraire des profils de la surface à n'importe quel endroit de celle-ci. Ces derniers peuvent être mesurés de manière détaillée. Leurs forme, profondeur et angles d'ouverture peuvent être déterminés.

Les analyses peuvent également inclure des modifications de données de base pour travailler les images. Ce sont notamment les fonctions de filtre qui se prêtent bien au traitement des données pour rendre les gravures plus lisibles.

Par un calcul spécifique, il est possible de séparer ou mettre en valeur l'une ou l'autre des deux caractéristiques d'une surface que sont la rugosité et l'ondulation. Comme son nom l'indique, la rugosité se compose des microformes situées sur la surface qui provoquent une texture particulière. L'ondulation, quant à elle, représente la forme générale de la surface. Lorsque l'on convertit les différents résultats en image 3D on aperçoit la différence. Une image qui met en avant la rugosité paraît plutôt plate et rugueuse tandis que celle qui favorise l'ondulation montre des dénivelés plus forts avec une surface plus lisse.

Les images filtrées ne contiennent plus qu'une fraction des informations des surfaces réelles. Comme nous l'avons vu, le filtrage atténue ou supprime un des caractères de la surface (ondulation ou rugosité) au profit de l'autre. Bien qu'il s'agisse d'une image en quelque sorte faussée, cette fonction de traitement de données peut être très utile pour déchiffrer les gravures par exemple (voir infra).

\section{La tribologie: étude quantitative}

Si nous pouvons, dans le cadre d'une étude qualitative, varier de manière assez considérable les échelles de mesure, le deuxième protocole, celui de l'étude des microsurfaces, demande un paramétrage plus précis autant dans l'acquisition que dans le traitement et l'analyse des images et données 3D.

L'acquisition des données se déroule pour toutes les mesures avec la même configuration du dispositif de mesure (crayon de $300 \mu \mathrm{m}$ ) et un paramétrage identique (pas de $0,469 \mu \mathrm{m}$ ).

Le traitement des données est, comme l'acquisition, réalisée avec un souci d'homogénéité. Pour les fenêtres mesurées et analysées, il est indispensable que l'image soit sans défaut de mesure: il est notamment nécessaire qu'aucun point non mesuré ne se trouve dans la fenêtre mesurée. Chaque surface est d'abord redressée; puis, pour les calculs, un zoom est effectué afin de créer une image de $150 \mu \mathrm{m}$ de côté. Il devient alors possible de procéder à des analyses qui seront par la suite comparables et reproductibles. Une des premières opérations consiste à calculer des paramètres standards.

Dans la métrologie appliquée aux surfaces, on se sert en effet de différents calculs pour la caractérisation quantitative. Pour notre étude, nous avons seulement pris en compte des paramètres du groupe I (d'après les normes de microtopographie). Ceux-ci regroupent uniquement des paramètres d'amplitude. En effet, l'ensemble de ces paramètres est défini par rapport à un plan moyen obtenu par un redressement selon le plan des moindres carrés de la surface mesurée, puis par centrage des altitudes autour de la moyenne. Il s'est avéré que les paramètres « St » et « Sv » étaient particulièrement intéressants pour caractériser les états de surfaces sur les calcaires que nous avons analysés. La valeur «St » représente la hauteur totale de la surface, c'est-à-dire la hauteur entre le pic le plus haut et le creux le plus profond. « $\mathrm{Sv}$ » exprime la profondeur de la plus profonde vallée de la surface. Il s'agit en réalité de la valeur qui désigne la profondeur entre le plan moyen et la vallée la plus profonde.

Cette démarche a été utilisée pour la distinction des roches susceptibles d'avoir été utilisées comme support de gravure à la Marche mais également pour la caractérisation d'usures et de polis sur les surfaces gravées (voir infra).

Un autre moyen que l'on peut mettre en ouvre dans la caractérisation de microsurfaces est la distribution des pics. Il s'agit d'une étude statistique qui calcule la répartition de pics selon la profondeur d'un plan de coupe horizontal. Le résultat apparaît sous forme d'un histogramme. La méthode de calcul est la suivante: pour un nombre déterminé de « tranches » de profondeur, on coupe la surface par un plan horizontal, appelé plan de coupe, et on compte le nombre de pics (c'est-à-dire tous les éléments de la surface à convexité, ce qui inclut les bosses) situés au-dessus du plan. Le nombre de tranches correspond au nombre des colonnes dans l'histogramme (fig. 25). On répète l'opération du point le plus haut jusqu'au point le plus bas de la surface mesurée. 
Cette distribution donne une idée de la répartition des altitudes des pics et, ainsi, de l'homogénéité ou non de la surface. Cette dernière est liée à la granulométrie de cette surface. La valeur absolue des pics comptés ne donne cependant qu'une approximation des amplitudes et dimensions réelles car les seuils, c'est-à-dire les plans 0 des surfaces, ne sont pas parfaitement identiques entre eux. En effet, chaque seuil dépend de l'amplitude relative de chaque mesure. Cependant, le type de distribution des pics peut nous fournir des informations sur la morphologie des surfaces. Il est donc nécessaire de se concentrer lors de cette étude uniquement sur les $\Delta$ entre les colonnes dans l'histogramme.

Reste à noter que les mesures de microsurfaces permettent, en plus des études quantitatives, de générer des visualisations qui illustrent les surfaces et leur morphologie. Ainsi, comme pour les études de la forme des gravures, les images en fausse couleur, en simulation photographique ou l'extraction de profils peuvent être utiles pour analyser les surfaces, notamment dans le cadre d'une analyse comparative.

\section{ÉTUDE DES PIERRES GRAVÉES DE LA MARCHE}

Les approches et techniques expliquées ci-dessus et qui ont été appliquées sur les pierres gravées de la Marche nous permettent d'obtenir de nouvelles informations sur ces supports riches et originaux en ce qui concerne les représentations et les comportements symboliques préhistoriques.

\section{L'ICONOGRAPHIE}

La plupart des pierres sont fragmentaires. Parfois, l'éclatement a réduit les supports gravés à l'état de fragments de taille très réduite, qui ne laissent apparaître que quelques traits. Par conséquent, en ce qui concerne l'identification des figurations sur les 1409 pierres que nous avons étudiées, il faut noter au préalable que la liste des motifs est sans doute incomplète. Un nombre considérable de pierres est donc susceptible de porter encore d'autres figures qu'il reste à déchiffrer.

Lors de la présentation des résultats, l'aide précieuse que nous a apportée l'utilisation des images de synthèse apparaîtra dans les exemples concernés. Nous tenons d'emblée à souligner que le déchiffrement et la lecture de certains supports n'auraient guère été possibles sans cette technique.
Nous allons d'abord nous intéresser aux motifs intelligibles, figuratifs et abstraits qui ont été découverts dans le cadre des travaux successifs sur le site. Notons cependant la présence de traits indéterminés qui n'ont pas pu être attribués à des motifs spécifiques. Ces gravures sont en nombre considérable. Elles occupent une place particulière dans le domaine de la gravure sur pierre, particulièrement abondante au Magdalénien et seront par conséquent abordées ultérieurement.

\section{LES ANIMAUX}

Le bestiaire de la Marche est très riche. Le nombre des animaux domine le corpus par rapport aux figurations humaines. On trouve des animaux typiques du bestiaire représenté au Magdalénien moyen tels que des rennes, des mammouths mais aussi des bisons, des ours et des chevaux. Les félins ont également, bien que plus rarement, été représentés par les graveurs de la Marche. Certains animaux présents sont cependant plus inhabituels pour l'iconographie magdalénienne. Ainsi existe-t-il à la Marche plusieurs représentations de léporidés.

Ce sont d'abord les travaux de L. Pales qui ont montré cette richesse iconographique des figures animales de la Marche. La plupart du temps, les figures sont d'une très belle facture et leur attribution ne pose pas de problème. Malgré tout, vingt animaux restent indéterminés car la forme de l'animal ne permet pas de préciser l'espèce ou le genre représentés. Au final, sur les 369 pierres qu'il a remontées, relevées et étudiées, L. Pales a identifié plus de 200 animaux qui proviennent de 8 familles différentes: équidés, bovinés, ovicaprinés, félidés, ursidés, cervidés, léporidés et proboscidiens (fig. 8).

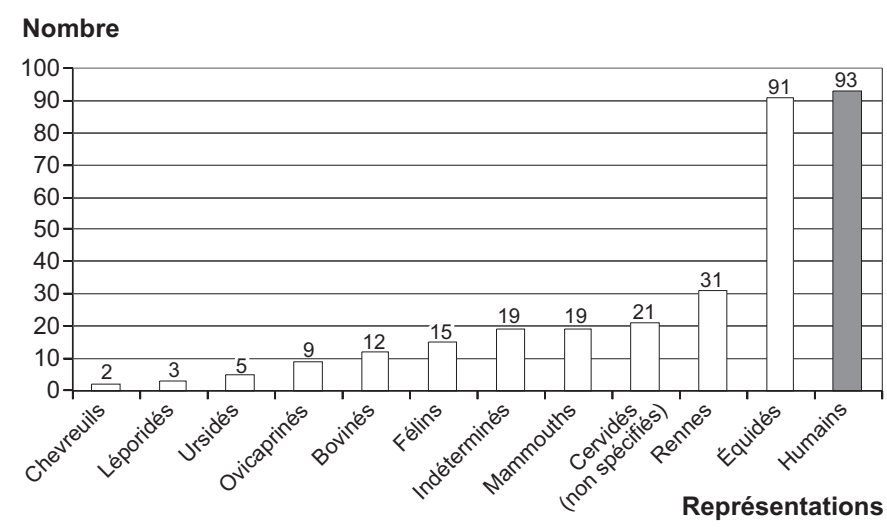

Fig. 8 - Motifs figurés sur les pierres de la Marche, inventaire de l'étude Pales (d'après Pales, Tassin de Saint-Péreuse, 1969, 1976, 1981, 1989). 
Les représentants de ces familles ne sont pas présents en nombre égal. À la première place se trouvent les chevaux (Equus sp.), seuls représentants de la famille des équidés. Ils sont 91 à être gravés sur 64 supports. Les cervidés, avec au moins 54 individus représentés, occupent la seconde place de ce classement. Pour certains animaux, une précision a pu être faite quant à leur spécificité en fonction de leurs caractères anatomiques : ainsi existe-t-il de manière certaine 31 rennes (Rangifer tarandus) et 2 chevreuils (Capreolus capreolus) parmi les cervidés. Il est notable que ces chevreuils sont habituellement assez rarement déterminés dans le bestiaire représenté au Paléolithique; en cela le site de la Marche montre une fois encore son originalité iconographique. Il reste cependant 21 cervidés indéterminés, soit à cause du manque de détails représentés, soit à cause de l'état fragmentaire de l'objet.

Les félidés occupent une place particulière parmi les représentations animales. Bien qu'ils ne forment pas la famille la plus représentée, ils sont en nombre important, avec tout de même 15 individus.

Les bovidés gravés à la Marche sont au nombre de 21 dans les publications de L. Pales. Parmi ceux-ci, 12 bovinés ont été découverts.

Les ovicaprinés sont, eux, au nombre de 9 individus.

Les proboscidiens sont également assez fréquents. L'espèce des mammouths (Mammuthus primigenius) est représentée par 19 individus, gravés sur 18 supports. Une dalle, notamment, porte la gravure de 2 mammouths affrontés (fig. 9).

Parmi les familles plus rares, les ursidés, dont L. Pales a identifié 5 représentations, sont à mentionner

Une autre famille, tout à fait exceptionnelle dans l'iconographie paléolithique, est celle des léporidés. En effet, L. Pales a répertorié 3 animaux appartenant à cette famille dont l'un est certainement un lièvre (Lepus sp.), les deux autres plutôt des lapins (Oryctolagus sp.). Chaque animal se trouve sur un support différent.

Les travaux qui ont succédé à ceux de L. Pales ont apporté d'autres figurations, notamment animales. Elles complètent ainsi l'image que l'on a de l'iconographie du site et ajoute quelques nouveaux éléments.

Dans son travail sur les pierres de la Marche D. Vialou a relevé quelques éléments pouvant appartenir à des figurations animales. Il a ainsi identifié de manière certaine la gravure d'un mammouth (Vialou, 1976).

Lors d'études plus récentes, J. Airvaux a montré la présence de nouvelles figurations sur des pierres inédites, issues des travaux de terrain entamés à la fin des années

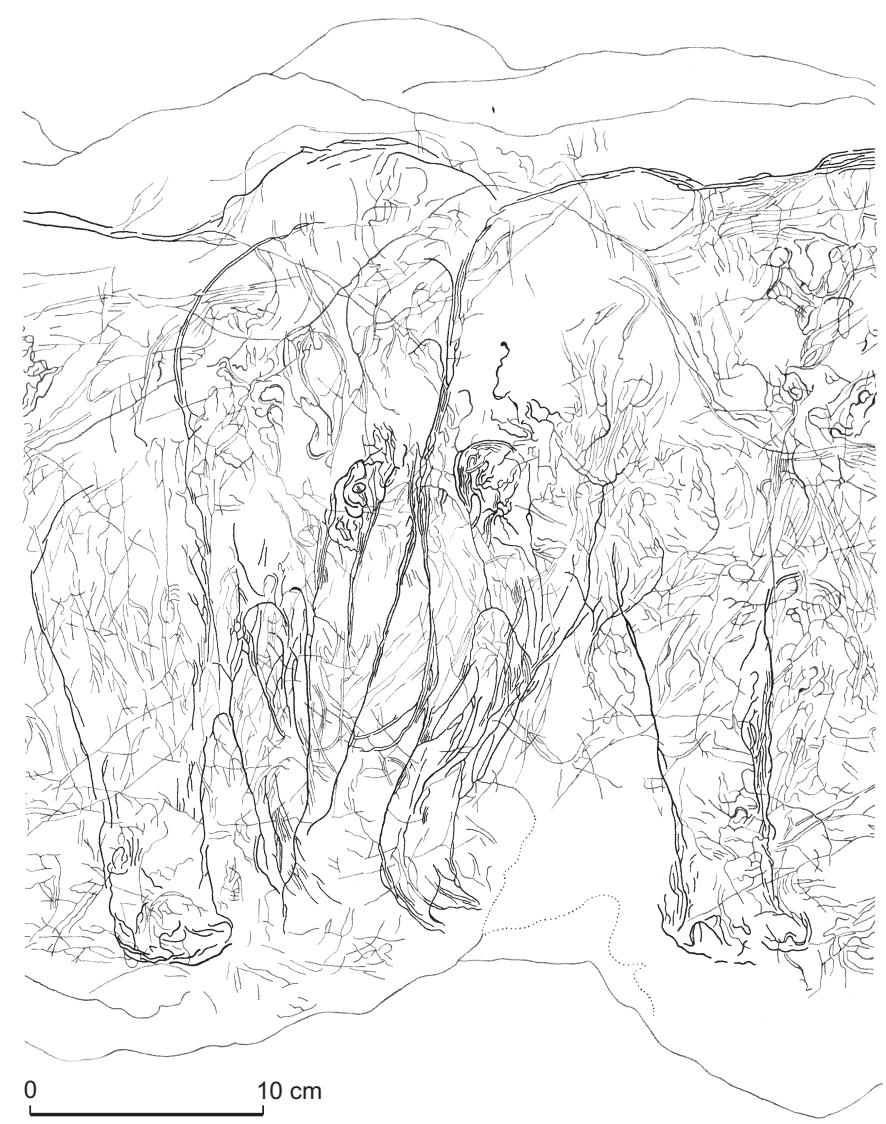

Fig. 9 - Détail du relevé d'une grande dalle de la Marche portant la représentation de deux mammouths affrontés (d'après Pales, Tassin de Saint-Péreuse, 1989).
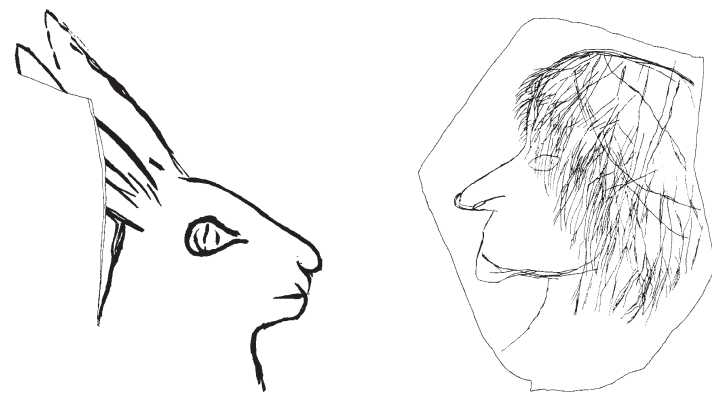

Fig. 10 - Deux figures relevées par J. Airvaux : à gauche, un lièvre; à droite, une tête humaine en profil gauche (d'après Airvaux, 2001). Sans échelle.

1990 (fig. 10). Au total, il a relevé 15 figures dont 4 animales (Airvaux, 2001). Elles montrent bien l'originalité de ce type de représentation qui est typique du site de la Marche: d'un point de vue stylistique, elles correspondent en effet aux motifs présentés par L. Pales. 


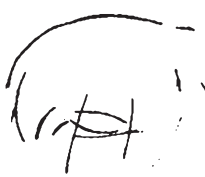

Ja 1319

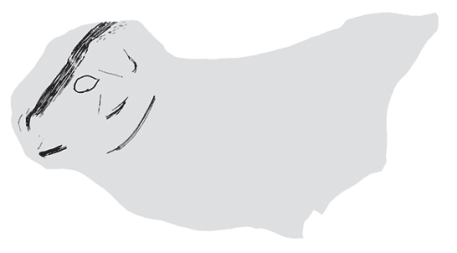

Ja 777

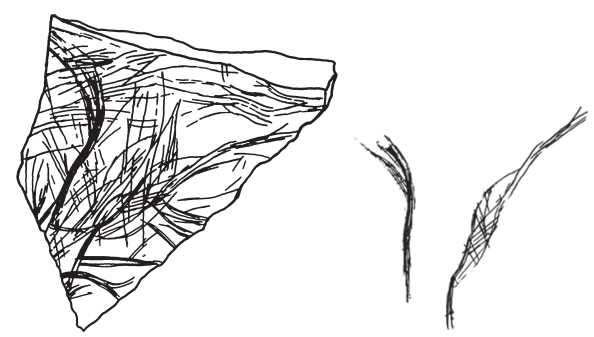

Ja 721

(cf. planches 40 et 41 )

Fig. 11 - Ensemble des figures indéterminées sur les pierres inédites de la Marche (relevés: N. Mélard). Sans échelle.

J. Airvaux a également trouvé sur une pierre la figuration d'un léporidé. Celle-ci est incomplète: il ne reste que la tête et nous ne pouvons dire précisément de quelle espèce il s'agit. La famille, cependant, ne peut être remise en doute. Cela porte à 4 le nombre de léporidés figurés à la Marche. Il est curieux de constater qu'ils semblent jouer un rôle assez important dans l'iconographie animale de la Marche, même s'ils restent marginaux par rapport aux espèces dominantes telles que les rennes et les chevaux. L'autre animal déchiffré par J. Airvaux est un félin, lui aussi assez rare sur d'autres sites (Airvaux, 2001).

Nos propres travaux sur les pierres inédites ont également révélé de nouvelles figures animales. Elles sont au nombre de 15 dont 5 indéterminées, notamment à cause de leur état

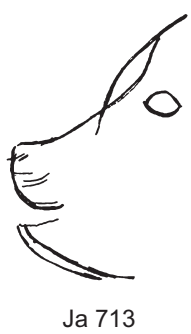

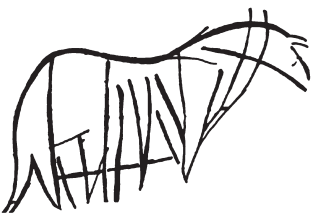

Ja 712

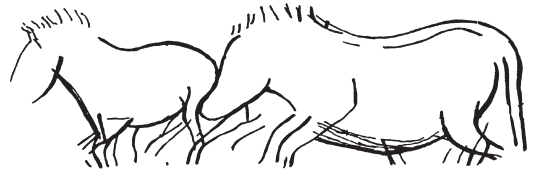

Ja 704

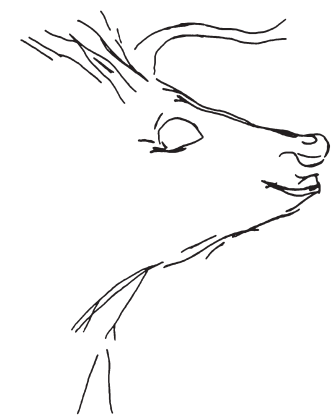

Ja 703

(cf. planches 24 et 25 )

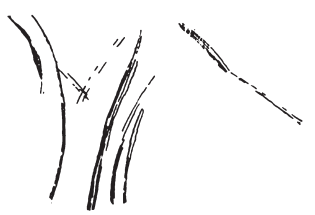

Ja 1272
Fig. 12 - Figures animales sur les pierres inédites de la Marche: un ours, en haut à gauche; trois chevaux, au centre; deux bovidés, en bas (relevés: $N$. Mélard). Sans échelle.

fragmentaire (fig. 11). Pour ce qui est des 10 animaux déterminés, il s'agit de 2 cervidés, de 3 chevaux, d'un boviné, d'un mammouth, d'un loup (Canis lupus), d'un phocidé (probablement un éléphant des mers, Mirounga angutrirostris) et d'un ours (Ursus sp.) (fig. 12 et 13). Certains de ces animaux, comme le loup et l'éléphant des mers, sont les premiers représentants de leur espèce dans l'iconographie de la Marche. Ils sont d'ailleurs en général très rares dans le bestiaire artistique du Magdalénien (Airvaux, Mélard, 2007).

Ces individus représentés de manière assez naturaliste et détaillée témoignent de la volonté du graveur de rester proche du modèle naturel. Pour le loup notamment, on remarque la forte élaboration du détail figuratif au niveau 


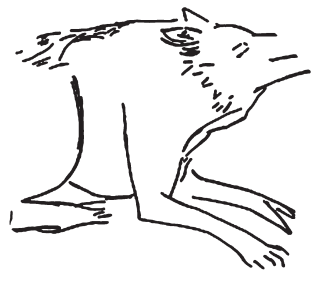

Ja 2

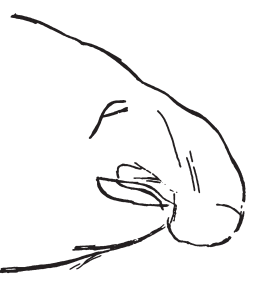

Ja 714 (cf. planches 36 à 39

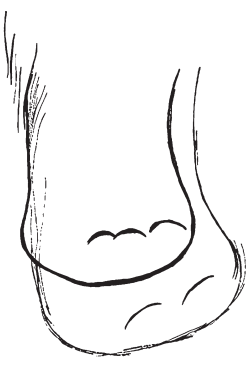

Ja 1361

(cf. planches 26 et 27 )

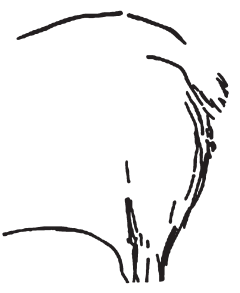

Ja 2

(cf. planches 36 à 39)

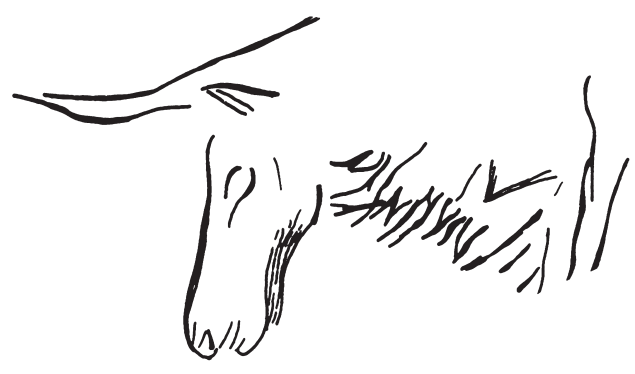

Ja 1181
(cf. planches 22 et 23)

Fig. 13 - Figures animales sur les pierres inédites de la Marche : un loup, en haut à gauche; un mammouth, en haut à droite; un éléphant des mers, au centre à gauche; deux cervidés, au centre à droite et en bas (relevés: N. Mélard). Sans échelle.

des pattes, des oreilles et des proportions du corps. De plus, un comportement particulier semble avoir été représenté, car l'animal n'est pas simplement debout mais couché, la tête tendue vers le haut. Ce style naturaliste apparaît également pour la plupart des autres figures animales que nous avons identifiées. Ce sont des animaux très bien dessinés, parfois jusque dans les détails. Ces réalisations sont d'une grande qualité technique. L'identification des espèces animales ne poserait donc aucun problème si ce n'était l'état incomplet des figures. Certains caractères anatomiques sont parfois figurés avec un soin particulier. Ce naturalisme trouve un
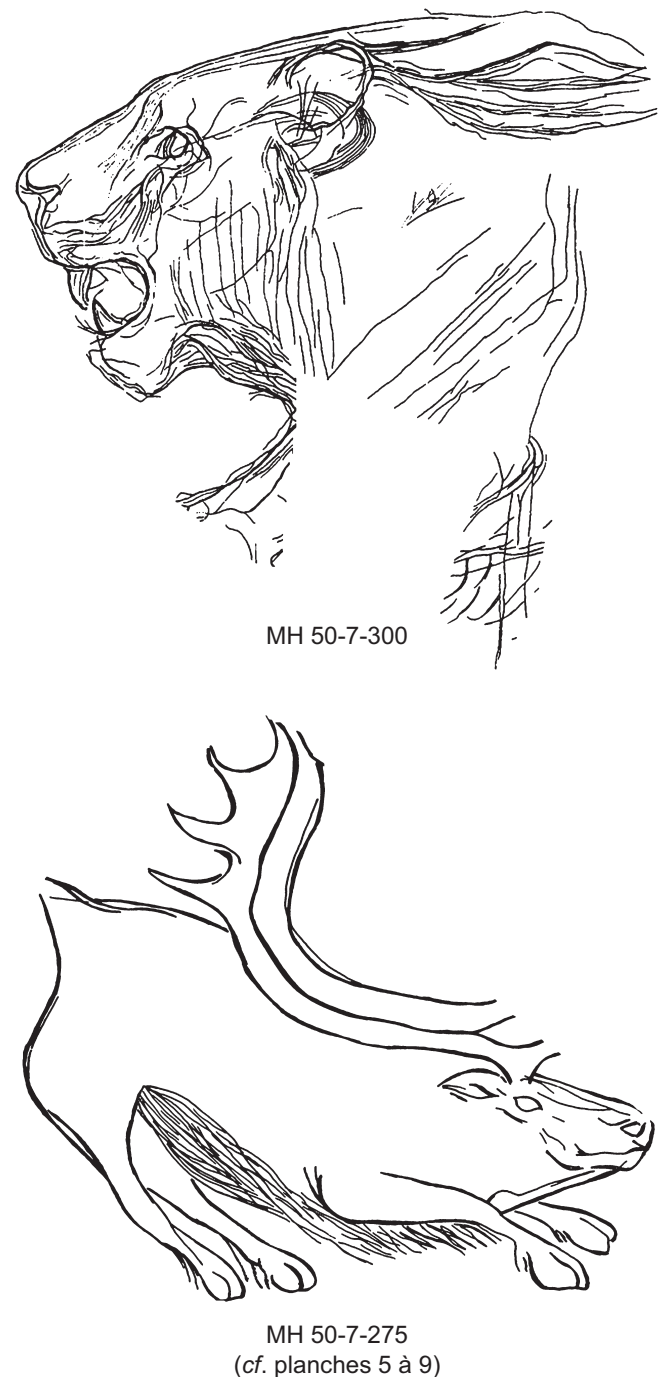

Fig. 14 - Figures animales (félin, en haut et renne, en bas) du site de la Marche, publiées par L. Pales et M. Tassin de Saint-Péreuse en 1969 et 1989. Étude reprise (relevés: N. Mélard). Sans échelle.

bon exemple avec la représentation d'un renne (MH507-275, pl. 5 à 9). Les yeux, les sabots ainsi que le pelage sont très élaborés (fig. 14). Pour cet exemple précis, nous devons ajouter que l'allure de l'animal est assez atypique. En effet, le cervidé se trouve dans une position dynamique: les membres antérieurs sont étendus vers l'avant tandis que la partie postérieure est debout. En conséquence, le dos présente une concavité exagérée. Un comportement précis est ainsi représenté avec cette figure. Il est par ailleurs notable que, même pour les figurations animales de taille assez réduite, il existe une recherche du détail. C'est par 
exemple le cas de la représentation d'un autre renne dont la tête mesure seulement $1 \mathrm{~cm}$ environ. Malgré ces dimensions, le graveur n'a pas hésité à représenter précisément les naseaux ainsi que les yeux et les oreilles (pl. 22 et 23).

Le style naturaliste est donc le plus fréquent dans le corpus des figurations animales; on trouve cependant un autre mode de représentation des animaux, remarquable parmi celles que nous avons étudiées. En effet, il existe des figurations juste esquissées. Parfois, ces figurations sont réduites à de simples silhouettes. C'est le cas du corps d'un animal indéterminé assez sommaire (Ja 1319, fig. 11) ou encore de la figuration d'un cheval dont la représentation se compose de quelques lignes seulement (Ja 712, fig. 12).

Bien que ces gravures soient sommaires, elles témoignent d'une technicité et d'une habileté des graveurs assez développées car les corps des animaux sont représentés de manière bien proportionnée.

Les animaux sont enfin plus ou moins complets : parfois un animal initialement complet, tel que le loup, a été fragmenté et rendu incomplet, parfois, comme c'est le cas pour la tête du boviné, l'animal n'a jamais été figuré de manière complète (Ja 703, fig. 12 et Ja 2, fig. 13). Lorsque des éléments anatomiques sont interrompus par les bords des fragments, les informations restent souvent insuffisantes pour savoir si l'animal était ou non complet à l'origine. Ceci est par exemple le cas pour la patte de mammouth gravée sur un bloc (pl. 26 et 27).

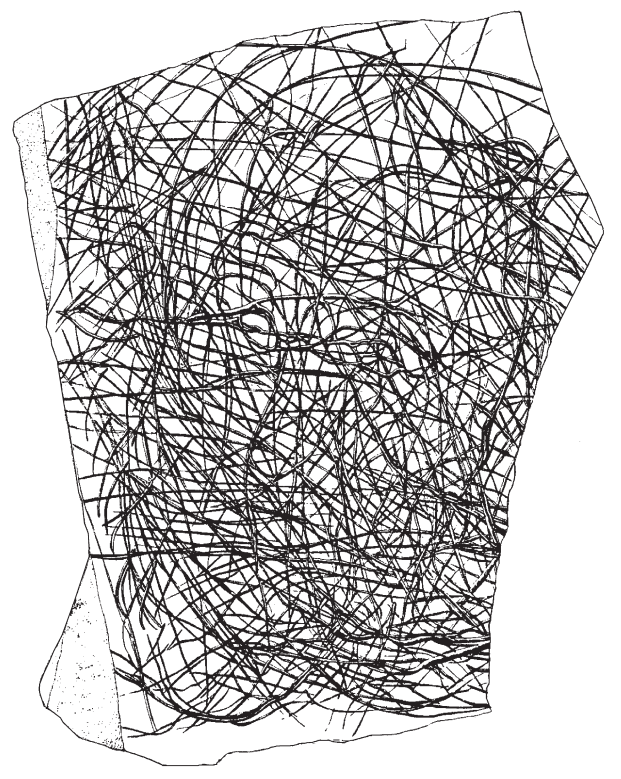

\section{LES HUMAINS}

Les représentations humaines sont sans doute une des originalités les plus marquantes du site de la Marche. L. Pales a publié 93 figurations humaines, dont des têtes isolées et des corps complets ou incomplets, céphales ou acéphales (fig. 15). J. Airvaux a, pour sa part, ajouté 11 humains à ce corpus (fig. 10 et 16). Nos propres travaux ont également mis en évidence des figurations humaines:
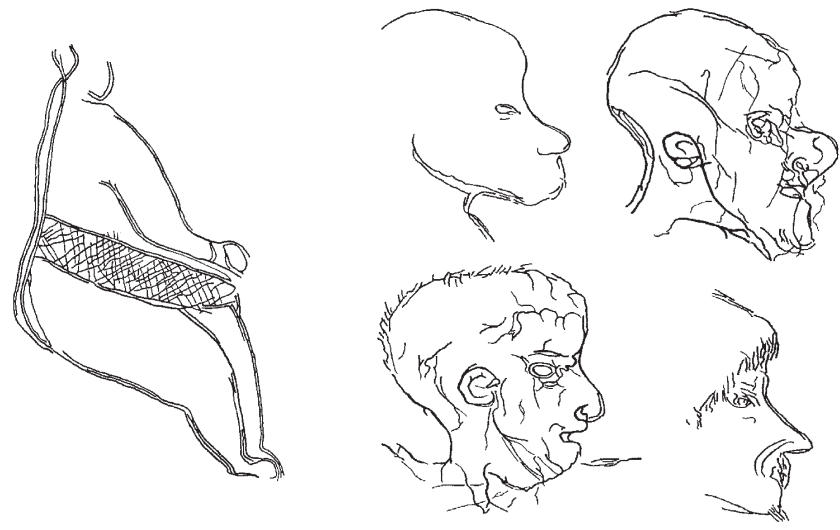

Fig. 15 - Relevés sélectifs de figures humaines identifiées par L. Pales (d'après Pales, Tassin de Saint-Péreuse, 1976). Sans échelle.

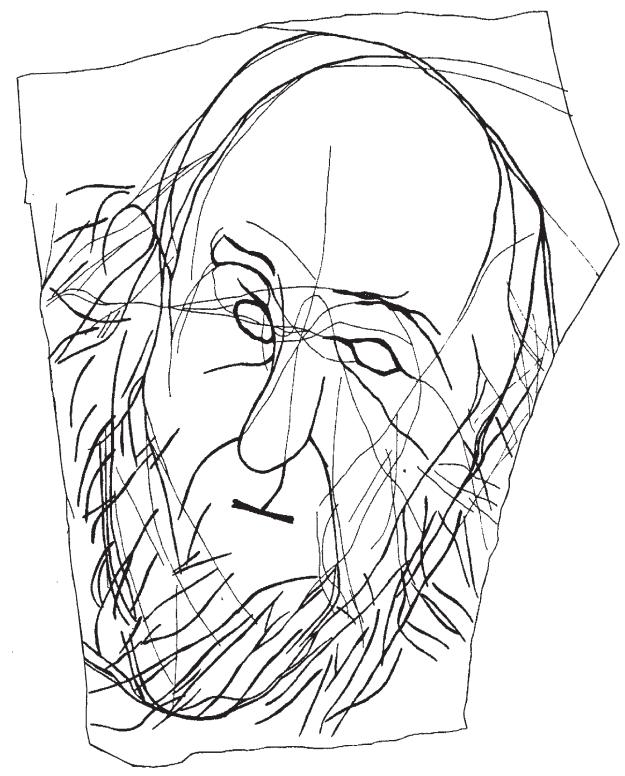

Fig. 16 - Tête humaine vue de face et dissimulée dans un palimpseste de traits : relevé complet à gauche et sélectif à droite (d'après Airvaux, 2001). Sans échelle. 
elles sont au nombre de 6 . Ainsi le corpus des humains de la Marche comprend actuellement 110 individus représentés. Les figurations récemment découvertes correspondent pour la plupart très bien à celles déjà décrites par L. Pales. Ce sont soit des figures complètes de personnages, assis ou debout, soit des représentations de têtes vues de profil. Une seule tête humaine est représentée de face (Airvaux, 2001).

Ces figures humaines sont d'une originalité étonnante quand on les compare aux représentations, la plupart du temps assez sommaires, que l'on connaît en général du Paléolithique supérieur. Les relevés que L. Pales a publiés illustrent la richesse de ces représentations aussi bien en termes de style que dans le mode de représentation et le niveau d'élaboration. En effet, de nombreux détails tels que les yeux, les cheveux, parfois même la parure, ont été figurés par les graveurs. Les figures humaines rejoignent souvent, de ce point de vue, la précision des figurations animales, du moins de celles qui sont très naturalistes.

Ce caractère naturaliste s'observe notamment dans la représentation des têtes humaines. Celles-ci donnent fréquemment l'impression d'être de véritables portraits, élaborés avec des détails surprenants. Le visage est pourvu d'yeux, d'une bouche et d'une frange. On distingue parfois même des coiffures.

L'exemple, sur une petite plaquette, de la gravure de deux profils humains regardant dans des sens opposés illustre bien le fait que les Magdaléniens élaboraient graphiquement des détails figuratifs. Les deux visages sont individualisés par la forme de leur profil et par l'indication de la coiffure (LP 22, pl. 18 et 19). Le personnage de gauche semble même porter une sorte de bandeau autour de la tête sur lequel est monté un objet qui pourrait être une plume (fig. 17). Sur la tête du personnage de droite, une forme gravée pourrait également correspondre à un élément de décoration. Les pièces récemment publiées par J. Airvaux montrent de la même manière à quel point les têtes humaines sont individualisées (fig. 10 et 16). Une fois de plus, le ou les auteurs des gravures ont porté une attention particulière aux détails de la coiffure.

Même pour des représentations de dimensions très réduites, nous observons un souci de précision étonnant (fig. 18). Deux petites têtes humaines qui mesurent à peine $1 \mathrm{~cm}$ de hauteur ont notamment été dessinées de manière très détaillée, avec la reproduction de leur bouche, de leurs yeux et de la coiffure pour une des deux (Ja 711, pl. 28 et 29) ; le graveur a aussi dessiné les cinq doigts de la main, longs de $1 \mathrm{~mm}$ seulement.
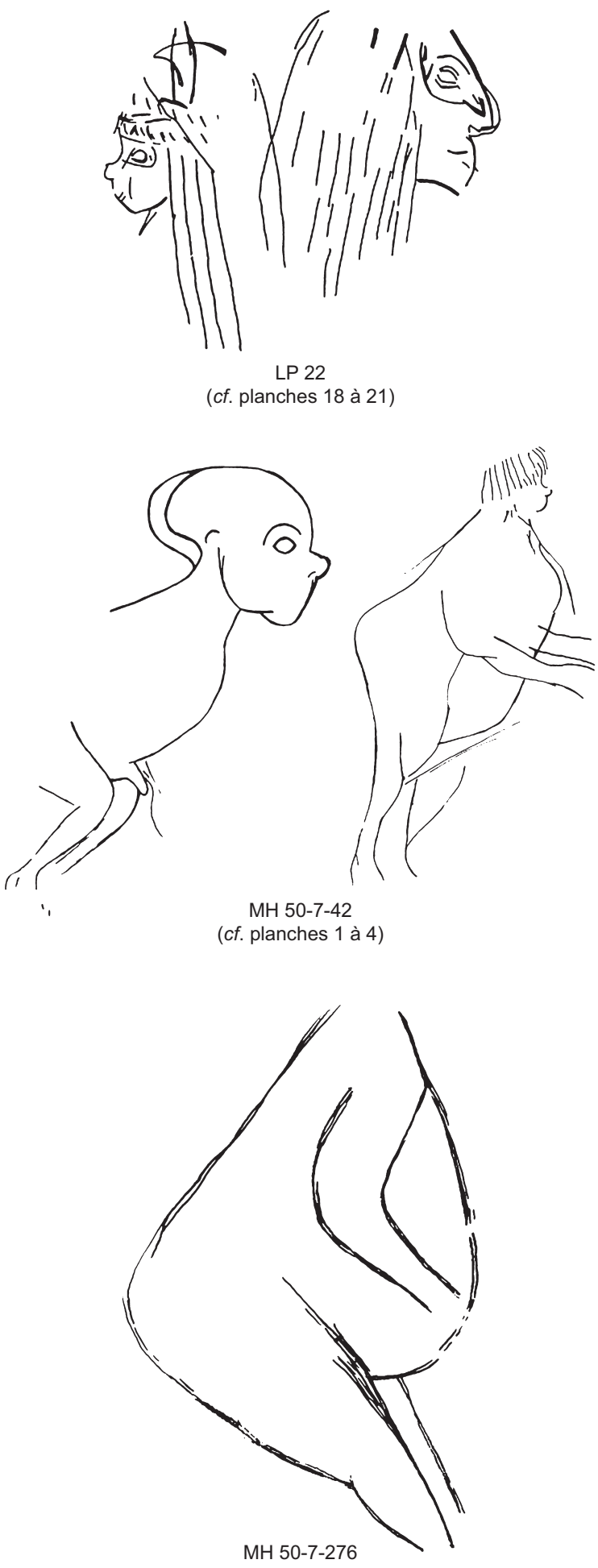

(cf. planches 10 à 15)

Fig. 17 - Cinq figurations humaines sur trois supports de la Marche, déjà publiées par L. Pales et Tassin de Saint-Péreuse en 1976 et reprises pour étude approfondie (relevés sélectifs: $N$. Mélard). Sans échelle. 


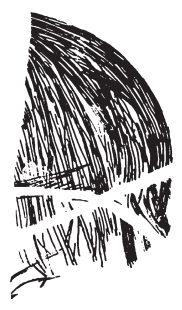

Ja 1115

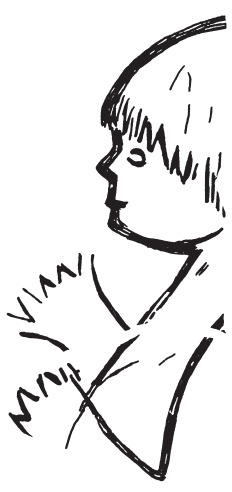

Ja 711 et Ja 587 (cf. planches 28 et 29)

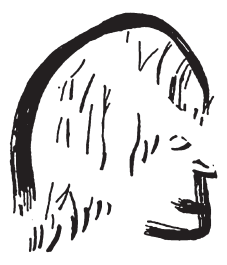

Ja 1

(cf. planches 55 et 56)

Fig. 18 - Figurations humaines inédites, sauf Ja 711 en partie publiée par J. Airvaux en 2001 (relevés sélectifs : N. Mélard). Sans échelle.

Parfois ces profils sont quasiment caricaturaux. Certains traits du visage sont exagérés, de préférence le nez, parfois le menton ou encore les arcades sourcilières, ce qui donne au profil un caractère très étrange. Sur une plaquette, sur laquelle se trouve également une femme assise qui est décrite ci-dessous et sur la même face qu'elle, nous avons identifié une tête humaine qui montre de manière claire cette modification caricaturale d'un profil humain (fig. 18).
Bien que les motifs soient diversifiés et individualisés, certains apparaissent à plusieurs reprises. C'est notamment le cas des figurations de femmes assises. Celles-ci occupent une place particulière, elles qui possèdent un ventre bombé et dont on présume pour cela qu'elles sont enceintes. En effet, L. Pales a pu montrer que cette forme du ventre est assez typique en cas de grossesse: cela a permis d'écarter l'hypothèse selon laquelle il pourrait s'agir de femmes obèses.

Nous avons étudié deux pierres qui montrent bien ce type de représentation de femmes (fig. 17). On s'aperçoit directement que les deux motifs répondent au même mode de représentation: les deux figures sont acéphales et sont assises avec les jambes parallèlement étendues vers l'avant. Certains détails sont cependant différents, tels que la position des bras. La femme gravée sur la plus petite plaquette a les coudes pliés, formant un angle d'approximativement $90^{\circ}$, et les bras levés. L'individu représenté sur la deuxième pièce semble poser ses mains sur son ventre. Celles-ci ne sont pas figurées, mais la position des bras laisse déduire la position des mains juste au niveau de la courbe du ventre.

Il est par ailleurs notable que les représentations féminines peuvent, sur certaines plaquettes, être réduites à la simple expression d'un détail anatomique, comme l'organe sexuel.

\section{LES VULVES}

Par notre étude sur le site de la Marche, nous avons pu mettre en évidence des figurations de vulves.

Nous avons séparé la présentation des vulves des figurations humaines mais également des figures abstraites pour la raison suivante: il est difficile de placer la représentation d'une vulve parmi les représentations humaines, car il s'agit de la réduction graphique de l'individu à un seul caractère morphologique, en l'occurrence l'organe sexuel féminin. Cependant, la représentation d'une vulve est une représentation figurative et non abstraite, et nous pouvons sans difficulté identifier celles de la Marche. Nous n'avons pas trouvé de représentations de triangles simples qui laisseraient apparaître un doute sur l'attribution du dessin: on ne peut donc pas confondre ces vulves avec de pures formes géométriques.

Dans les publications antérieures aux années 1990 de S. Lwoff, L. Péricard et L. Pales, il n'y a aucune mention de la représentation de vulves. Dans les années 1990, les travaux de J. Airvaux ont montré qu'il existe des représenta- 


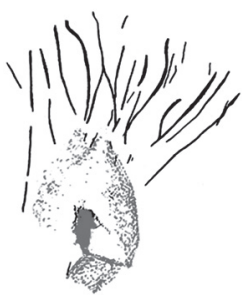

Ja 710 (cf. planche 32)

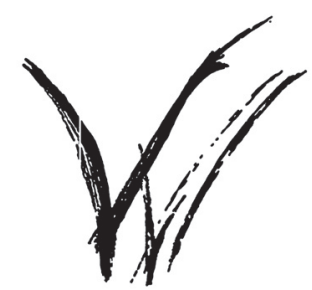

Ja 1287
Ja 711

(cf. planches 28 et 29)

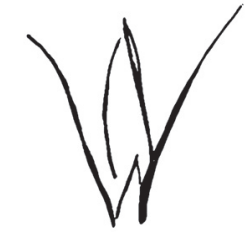

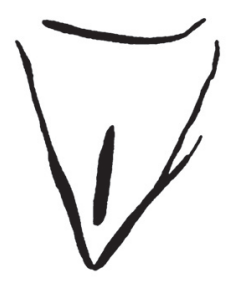

Ja 716

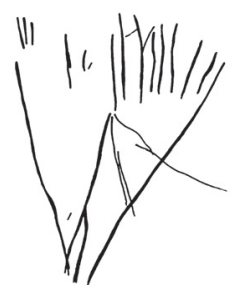

Ja 997

(cf. planches 30 et 31)
Fig. 19 - Figurations de vulves du site de la Marche : Ja 716 et Ja 711, relevés sélectifs publiés par J. Airvaux en 2001 ; Ja 710, Ja 997 et Ja 1287, relevés inédits (relevés : N. Mélard). Sans échelle.

tions de vulves sur les pierres de la Marche (Airvaux, 2001 et 2002). Nous avons, lors de notre étude, découvert d'autres représentations de ce motif et avons au final relevé 5 vulves, dont 2 qui avaient déjà été publiées par J. Airvaux (fig. 19).

Une des vulves est associée à une petite figure humaine (Ja 711, pl. 28). Elle est dessinée de manière assez détaillée. Les limites latérales du triangle pubien sont représentées par deux traits convergeant vers le bas sans pour autant se rencontrer. Ainsi une ouverture se forme-t-elle dans cette zone. Une seconde forme la surplombe d'ailleurs, et se compose de deux traits convergeant vers le haut. Une autre vulve possède le même style de représentation détaillée (Ja 997, pl. 30 et 31). Sur cette dernière, on remarque cependant un autre traitement de la partie supérieure du motif. En effet, les poils du pubis sont ici représentés par un alignement de traits parallèles.
D'autres représentations enfin, qui montrent des particularités dans le mode de représentation, ont été découvertes. L'une est la figuration d'une vulve dans laquelle le graveur a intégré le support naturel, qui forme la partie autour de l'orifice. Le reste du pubis est représenté par un ensemble de traits rayonnants qui forment un triangle (Ja 710, pl. 32). Une autre représentation de vulve est, elle, associée à une figuration humaine fragmentaire. Elle est dessinée de manière plus sommaire, par trois traits droits représentant les limitations droite et gauche du triangle pubien et la fente centrale.

Cette schématisation extrême d'un motif figuratif ne doit pas nous faire oublier qu'il existe aussi dans le corpus de la Marche de véritables abstractions.

\section{LES SIGNES ET FORMES ABSTRAITES}

Les travaux menés jusqu'à présent sur le site se sont en effet plutôt concentrés sur les motifs figuratifs, qu'ils soient animaux ou humains. Notre étude a cependant mis en évidence la présence de motifs composés à caractère abstrait.

$\mathrm{Au}$ total, nous avons retenu 14 cas où nous trouvons des représentations de formes abstraites plus ou moins complexes.

En premier lieu, nous avons identifié sur une pierre un fusiforme évasé avec des chevrons emboîtés à l'intérieur: celui-ci évoque une feuille d'arbre (fig. 20). Nous ne pouvons cependant pas attribuer de façon certaine une identification à cette figure gravée. L'absence de végétation dans l'iconographie paléolithique ne nous permet en effet aucune comparaison et, de ce fait, nous classons cette forme pour le moment dans les formes abstraites.

Un galet porte, quant à lui, un signe complexe en forme de quadrillage géométrique; ce dernier s'organise en trois bandes plus ou moins densément remplies de traits parallèles. Ce motif occupe la surface intégrale de la pierre et organise celle-ci en secteurs réguliers.

C'est un motif très original pour les représentations magdaléniennes notamment à la Marche où, jusqu'à présent, le corpus des représentations gravées se caractérisait surtout par les motifs figuratifs et les traits indéterminés.

Une autre forme, que L. Pales avait remarquée, concerne les «yeux isolés»(Pales, Tassin de Saint-Péreuse, 1989). Il s'agit d'ovales arrondis ou en forme d'amande qui se trouvent sur les surfaces gravées. Ces dessins évoquent en effet la forme d'yeux que l'on trouve normalement dans les figurations d'humains et d'animaux. Cependant, ceux-ci 


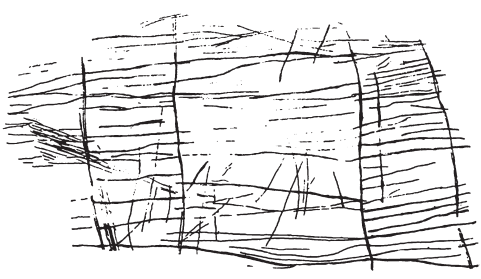

Ja 709

(cf. planche 54)

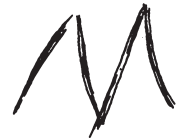

Ja 1160

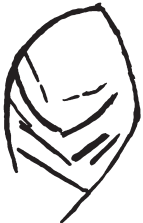

Ja 1217
Ja 706

(cf. planche 33)

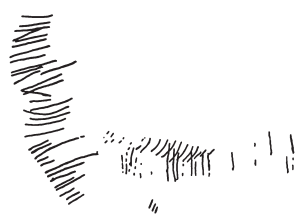

MH 50-7-276

(cf. planches 10 à 15)

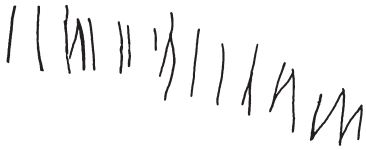

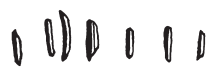

Ja 1383

Ja 1224

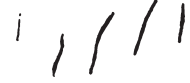

Fig. 20 - Formes abstraites inédites, gravées sur les pierres de la Marche : quadrillage, zigzag, "feuille », traits parallèles (relevés sélectifs :

N. Mélard). Sans échelle.

restent sans connexion graphique avec la figuration hypothétique d'une tête, d'où cette appellation de "yeux isolés ". Sur les pierres que nous avons relevées, nous avons repéré cinq de ces formes sur quatre supports. Leur morphologie est variable (fig. 21). Parfois nous avons l'impression de voir un œil de cervidé qui se caractérise habituellement par sa forme en amande et par un larmier marqué (Ja 1133, fig. 21). Dans d'autres cas, la forme est plus évasée et correspond plutôt à un œil de boviné (Ja 484, fig. 21) .

Un autre type de formes abstraites a également été identifié sur les pierres (fig. 20). Ce sont des groupements de traits parallèles plus ou moins longs qui ont été dessinés sur les surfaces. Les traits sont disposés de manière régulière et évoquent un certain rythme gestuel, ce qui nous permet d'écarter l'hypothèse de traces involontaires. Nous avons
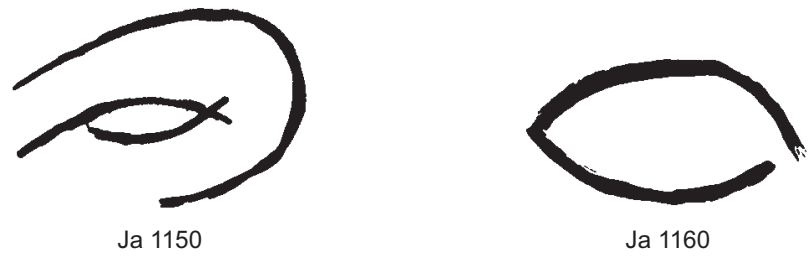

Ja 1160

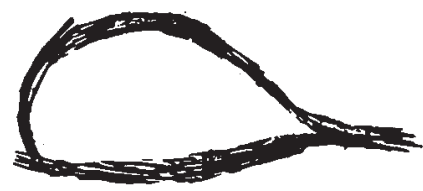

Ja 1133

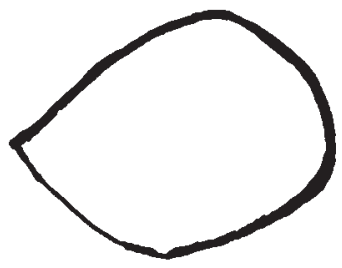

Ja 484

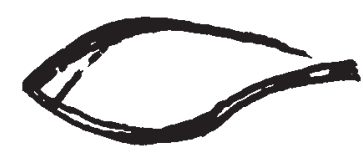

(cf. planches 42 à 44)

Fig. 21 - "Yeux isolés » inédits, gravés sur les pierres de la Marche (relevés sélectifs : N. Mélard). Sans échelle.

noté la présence de ce genre de séquences de traits parallèles sur six supports. Ils se trouvent soit de manière isolée sur les pierres, soit entremêlés avec d'autres entrecroisements de traits. Des associations avec des représentations figuratives, par relation graphique particulière, ont pu être faites dans deux cas. Le premier est celui d'un renne couvert de traits parallèles. Dans cet exemple, les traits dissimulent la figuration (pl. 22 et 23).

Sur cette même pièce, mais sur le verso, nous trouvons une autre forme abstraite: une série de lignes courtes ondulées et soigneusement emboîtées, longeant le bord de la pièce. La forme des gravures évoque le tracé typique d'une représentation de la ligne « dos-fesses-cuisses » des femmes schématisées telles que nous les avons trouvées sur un galet à la Marche (Ja 138, pl. 34 et 35). Le fait que ces formes répétées ne soient pas plus complètes et qu'elles soient emboîtées de cette manière ne nous permet cependant pas de les identifier comme figures féminines.

Enfin, pour compléter le corpus des réalisations abstraites présentes sur les pierres de la Marche, nous notons la présence d'un zigzag gravé de manière isolée sur la face d'une petite pierre (Ja 1160, fig. 20). La forme est gravée avec des traits assez marqués, qui ont nécessité plusieurs 
reprises, et constitue sans doute un signe volontairement représenté comme tel, c'est-à-dire de façon non figurative.

Dans le domaine des formes abstraites, des traits indéterminés, qui se trouvent parfois en densité considérable sur les pierres de la Marche, sont à mentionner. Même s'il est impossible de discerner une quelconque figuration dans ces entrecroisements, la nature intentionnelle de ces gravures est assurée. Il est donc clair que nous devons prendre en considération ces réalisations qui appartiennent au domaine des représentations abstraites.

Le site de la Marche comporte donc une richesse iconographique importante. Cependant, celle-ci n'est pas toujours facile à apprécier car souvent les figurations, qu'elles soient abstraites ou non, se superposent sur les mêmes surfaces. Leur lisibilité peut en être ainsi affectée, d'autant plus que le choix de la matière première joue également un rôle.

\section{LES MATIÈRES PREMIÈRES}

L'un de nos objectifs majeurs est d'étudier les supports gravés dans leur intégralité. C'est pour cela que nous nous sommes penchés sur les supports et les interactions entre l'outil et la surface. Avant d'aborder le questionnement propre à la morphologie des traces, il est nécessaire de s'attarder un moment sur la nature pétrographique des matières premières. D'un point de vue granulométrique, nous avons identifié deux types d'agencement bien distincts ainsi qu'un troisième qui est un mélange des deux premiers (fig. 22).

Le premier groupe comprend la majeure partie des pierres de la Marche (en noir sur la fig. 22). Il s'agit de roches qui se composent de grains de plus de $500 \mu \mathrm{m}$ en plus ou moins grand nombre et dont la granulométrie est souvent hétérogène. Près de la moitié des supports que nous avons étudiés possède ces caractéristiques granulométriques.

Le deuxième groupe (en blanc sur la fig. 22) se distingue par l'homogénéité et la finesse des grains, qui se situent autour de $100 \mu \mathrm{m}$ au maximum et ne dépassent jamais $500 \mu \mathrm{m}$. Seules 51 pierres présentent une telle granulométrie.

Un troisième groupe (en gris sur la fig. 22) réunit à la fois des caractéristiques du premier groupe (le mélange de plusieurs calibres de grains) et du deuxième (la finesse des grains). Il est également très important puisqu'il comporte plus de 470 pierres. Un litage de la roche est présent sur 117 supports, ce qui est un caractère supplémentaire pour les distinguer.

Pour approfondir nos connaissances sur la nature des supports, nous avons réalisé, par la microtopographie, des
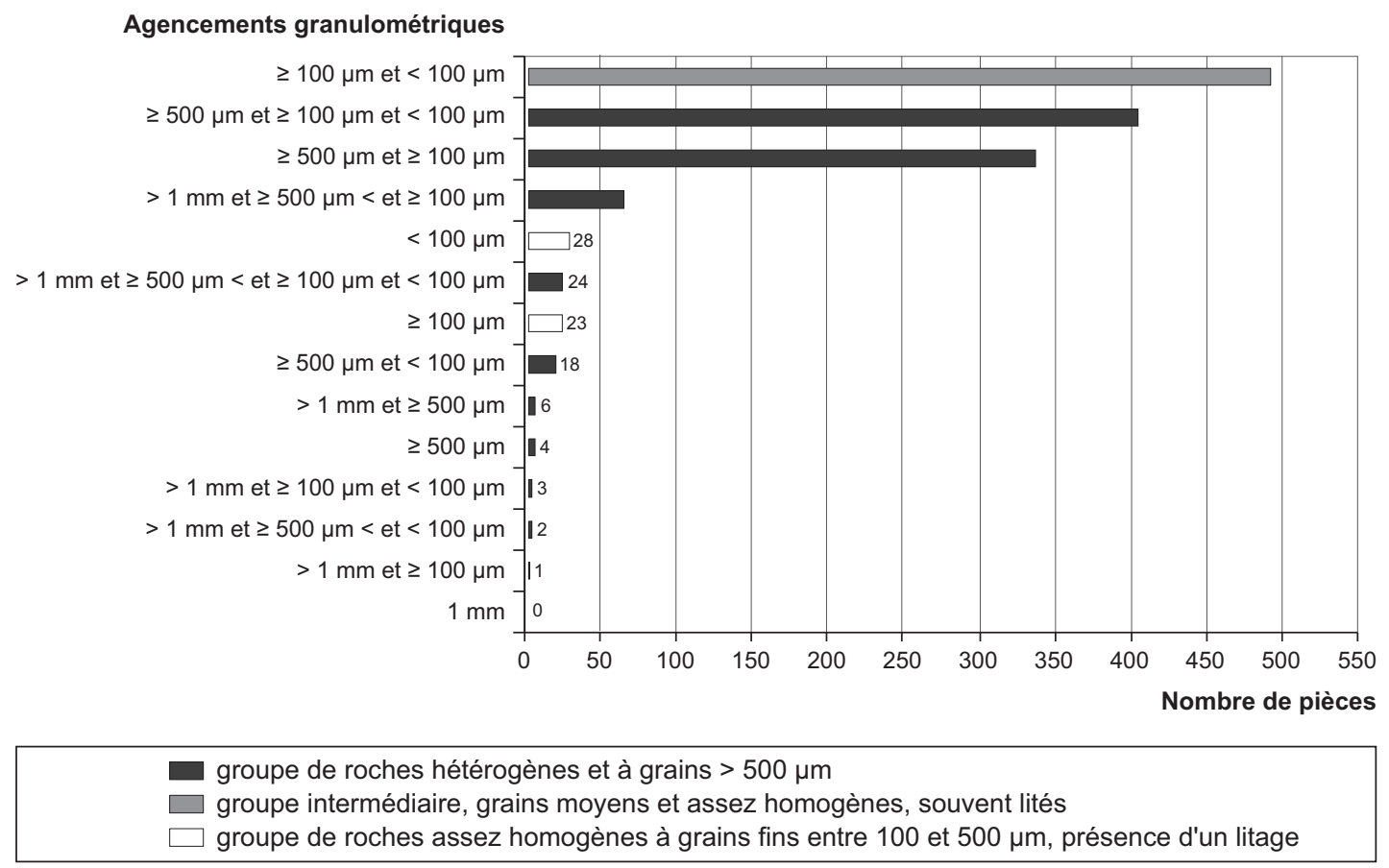

Fig. 22 - Répartition des faciès granulométriques des supports gravés de la Marche (sur un total de 1409 pierres). 

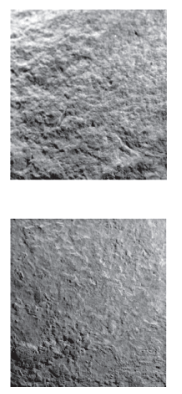
$1 \mathrm{~cm}$ $1 \mathrm{~cm}$

Fig. 23 - Macrophotographies du calcaire bathonien (en haut) et kimméridgien (en bas). Vue de dessus (à gauche) et de profil (à droite) (clichés : D. Bagault, Centre de recherche et de restauration des musées de France).

études plus ciblées sur un échantillon restreint de cet ensemble de pierres (voir infra). Il convient avant toute chose de définir notre démarche.

Comme nous l'avons mentionné précédemment, l'étude des microsurfaces cible la possibilité de caractérisation des surfaces calcaires afin de pouvoir distinguer différents faciès de roches, l'idée de base étant que chaque calcaire s'est formé sous des conditions bien spécifiques et que celles-ci ont influé sur sa morphologie. En effet, la surface d'une roche tout comme sa composition chimique dépendent de sa mise en place et ainsi de l'étage géologique auquel elle appartient.

Notre travail a donc d'abord consisté à tester cette hypothèse sur des roches susceptibles d'avoir servi de supports pour la gravure dans le site de la Marche. Nous avons ensuite effectué des mesures sur les échantillons archéologiques pour comparer les paramètres de surfaces archéologiques gravées et non gravées avec les résultats issus des échantillons géologiques.

L'application de cette démarche sur les pierres de la Marche nous confirme la présence de faciès de roches bien distincts parmi les pierres gravées du site. La première variété est le calcaire bathonien. La deuxième variété est le calcaire kimméridgien qui a été prélevé en contexte géologique dans une coupe ou ramassé sur un champ.

D'un point de vue morphologique, aucun autre calcaire de la région ne semble susceptible d'avoir été présent à la Marche. En effet, nous avons échantillonné d'autres affleurements calcaires représentant des étages géologiques différents. Il s'est cependant avéré, dès l'observation à l'œil nu, que ces calcaires ne correspondent pas aux plaquettes étudiées à la Marche. Le calcaire oolithique de la région de
Chauvigny, par exemple, montre une morphologie macroscopique complètement différente qui n’a pas été repérée dans le site.

Le calcaire bathonien est donc la roche dominante dans les environs de Lussac-les-Châteaux. Ses caractéristiques morphologiques sont bien spécifiques. Ce calcaire est peu ou très irrégulièrement lité (fig. 23). À l'œil nu, nous observons les composants les plus grossiers de la matrice rocheuse. Ces grains ont en conséquence une taille supérieure à $0,1 \mathrm{~mm}(100 \mu \mathrm{m})$. Leur taille peut atteindre $500 \mu \mathrm{m}$ dans certains cas exceptionnels. Ces grosses particules ne sont cependant pas fréquentes et n'apparaissent que ponctuellement sous la forme de macrodébris de fossiles. Le reste de la matrice rocheuse possède plutôt un grain fin dont la taille est inférieure à $100 \mu \mathrm{m}$.

Certaines pierres montrent une porosité assez importante de quelques zones, avec des cavités supérieures à $300 \mu \mathrm{m}$ et atteignant parfois $1 \mathrm{~mm}$ de diamètre.

L'étude des relevés microtopographiques nous apporte des informations supplémentaires pour la caractérisation de la surface.

La surface se caractérise par une ondulation assez conséquente. En effet, une image filtrée supprimant sa rugosité nous montre des dénivelés de plus de $400 \mu \mathrm{m}$. Ce sont donc de "grandes formes » qui donnent les caractères principaux de la surface: il s'agit sans doute de microfossiles marins et de leurs fragments. À cette forme générale s'ajoute une rugosité qui présente également des microformes assez marquées, formant des creux de $300 \mu$ m environ au maximum.

Le profil typique de la surface du calcaire bathonien montre bien, une fois encore, que cette roche se caractérise autant par l'ondulation de sa surface que par sa rugosité (fig. 24).

Nous voyons que, par rapport au plan moyen d'une surface, les pics peuvent dépasser celui-ci de $300 \mu \mathrm{m}$, voire un peu plus. Parallèlement, les profondeurs tombent jusqu'à presque $300 \mu \mathrm{m}$. Ceci crée une différence de marche très importante de plus de $600 \mu \mathrm{m}$.

Pour avoir une idée plus précise encore de la composition de la surface, nous avons étudié la distribution des pics (fig. 25). Nous observons de manière systématique une dispersion des pics assez hétérogène. En effet, les histogrammes des distributions montrent aussi bien des groupements à deux concentrations majeures que des distributions avec un groupement majeur. De plus, nous remarquons une dispersion de hauteur de pics très variable, entre $75 \mu \mathrm{m}$ et $25 \mu \mathrm{m}$, pour les pics les plus bas et les pics les plus hauts. 

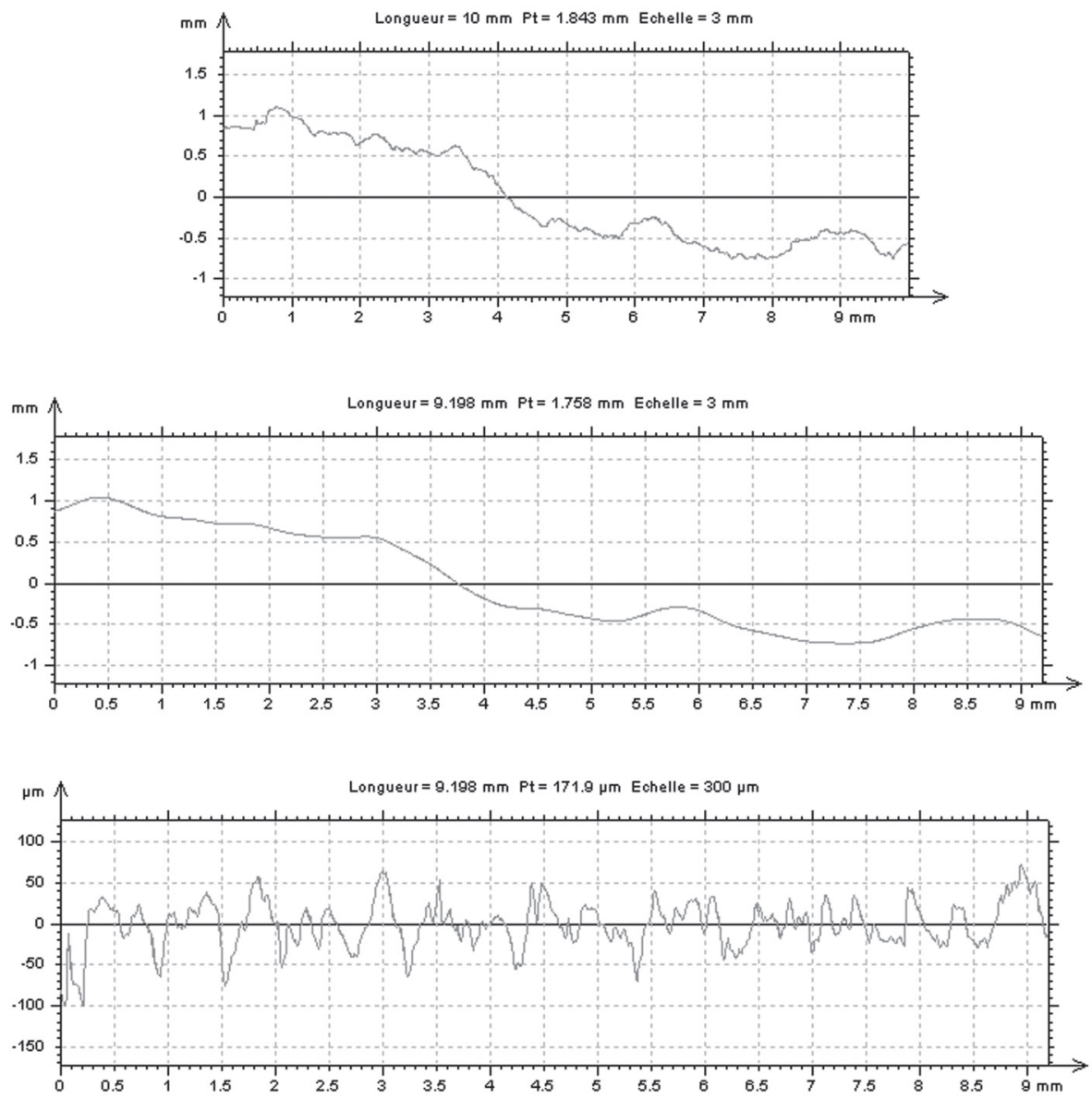

Fig. 24 - Profils d'une surface de calcaire bathonien : sans filtrage (en haut), ondulation (au centre), filtrage de rugosité gaussien (en bas).

L'intégralité des observations nous indique formellement l'hétérogénéité des surfaces.

L'analyse microtopographique effectuée sur l'autre calcaire, le kimméridgien, montre à quel point les deux faciès peuvent différer.

Le calcaire kimméridgien affleure seulement dans une zone assez restreinte, à l'ouest de la ville de Châtellerault. Ce calcaire est systématiquement agencé en couches très régulières dans les affleurements et il se détache selon des plans de clivage parallèles. Les pierres détachées sont en conséquence des plaques et plaquettes très régulières. De même, nous trouvons dans les champs des supports plans et réguliers en forme de plaquettes. Comme pour le calcaire bathonien, nous avons procédé à une étude morphologique, d'abord de manière descriptive à l'échelle macroscopique, puis de façon approfondie par une analyse de la microtopographie des échantillons.
Le profil de l'échantillon nous montre un litage très régulier avec des couches parallèles au plan de clivage (fig. 23). Ce calcaire se caractérise ainsi par une surface très homogène et plane. Néanmoins, celle-ci n'est pas tout à fait lisse mais présente un aspect légèrement granuleux. Il est cependant difficile d'observer les particules constituant la surface de manière individuelle à l'œil nu. Ceci nous indique que de la plupart des constituants de cette roche doivent avoir une taille inférieure à $100 \mu \mathrm{m}$.

Si l'on observe la microtopographie de ce calcaire, nous constatons d'emblée un aspect assez granuleux de la surface. Mais, dès les premières études microtopographiques, nous nous apercevons que l'amplitude des creux et pics est moins élevée que dans le cas du calcaire bathonien. Les amplitudes les plus extrêmes de la surface ne dépassent pas $200 \mu \mathrm{m}$, soit la moitié des valeurs observées sur les surfaces du calcaire bathonien. 

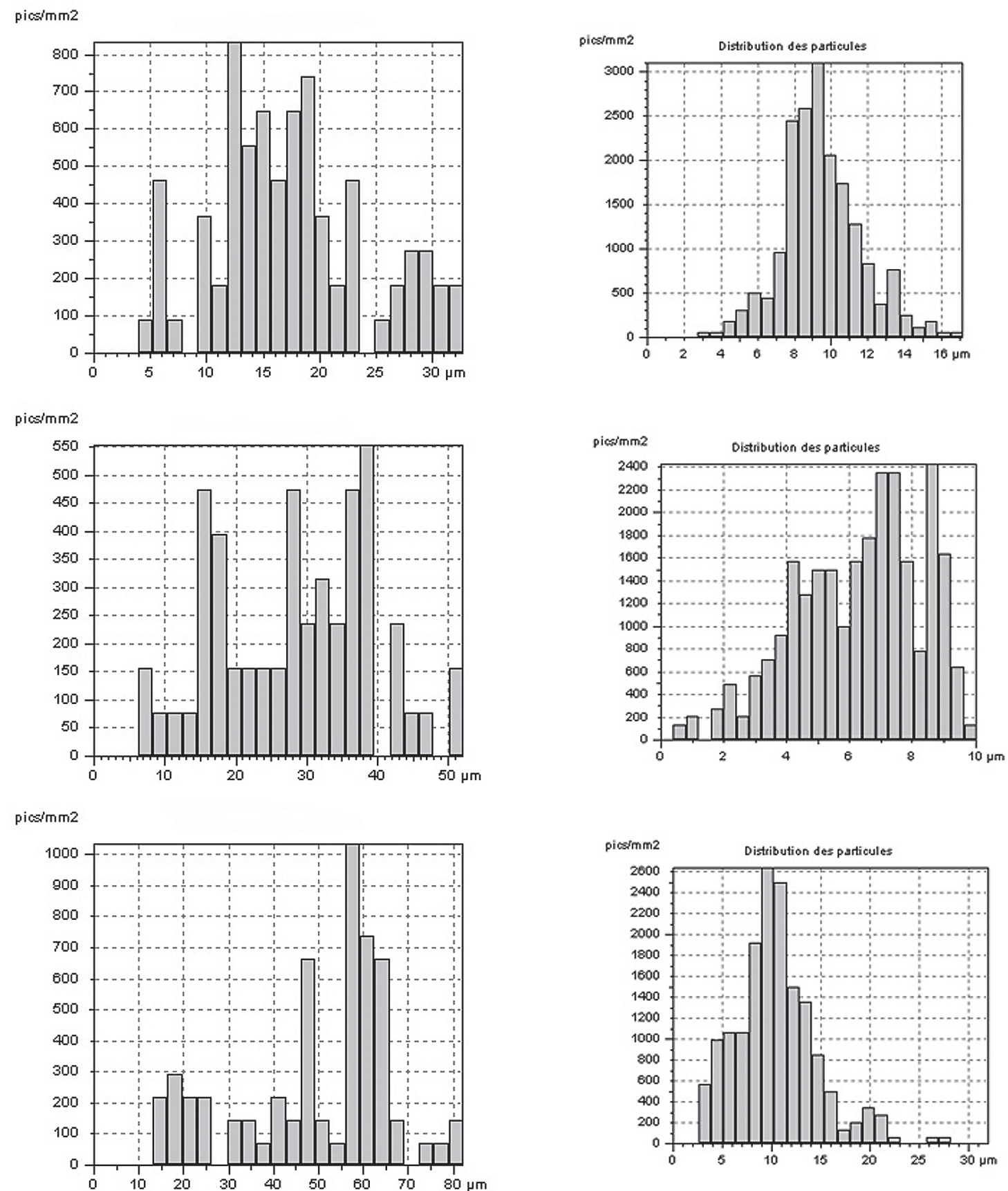

Fig. 25 - Comparaisons de la distribution des particules sur un calcaire kimméridgien (à droite) et un calcaire bathonien (à gauche).

Une seconde observation s'appuie sur l'étude des profils (fig. 26). La surface est marquée par une rugosité assez conséquente et se caractérise par de nombreux petits pics. En ce qui concerne les alternances des "grandes formes", on voit que les amplitudes sont beaucoup moins fortes. Dans notre exemple nous notons, par rapport au plan moyen, des hauteurs qui ne dépassent pas $70 \mu \mathrm{m}$ et des profondeurs qui ne sont pas inférieures à $60 \mu \mathrm{m}$. Cette marche maximale de $130 \mu \mathrm{m}$ contraste avec les valeurs mesurées sur les surfaces du calcaire bathonien qui étaient de l'ordre de $600 \mu \mathrm{m}$.

Pour ce qui est de la rugosité de la surface, nous devons observer la distribution des pics mesurés sur les micro- 

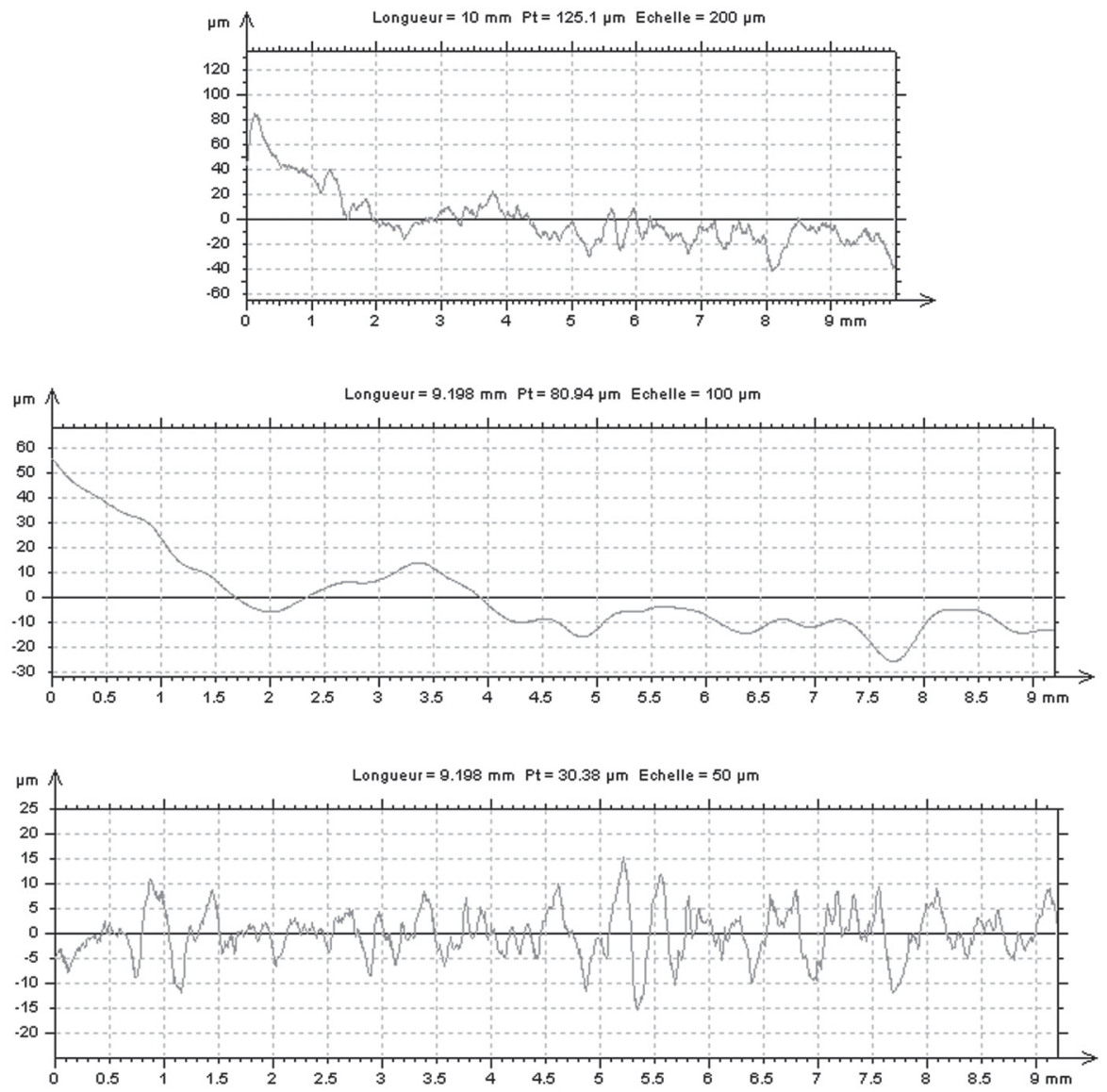

Fig. 26 - Profils d'une surface de calcaire kimméridgien : sans filtrage (en haut), ondulation (au centre), filtrage de rugosité gaussien (en bas).

surfaces $(0,25 \mathrm{~mm} \times 0,25 \mathrm{~mm})$. Les histogrammes qui en résultent montrent un assemblage de pics beaucoup mieux classés que sur les surfaces du calcaire bathonien (fig. 25).

La plupart du temps, les surfaces sont dominées par un groupement de pics. Dans certains cas, les pics de la distribution sont un peu plus étalés sans pour autant atteindre la distribution des pics du calcaire bathonien. Ce que nous observons de manière systématique, c'est que la variation des amplitudes se situe dans une marge beaucoup plus restreinte que dans le cas du calcaire bathonien. Nous mesurons des marges maximales d'environ $25 \mu \mathrm{m}$ mais, souvent, la fourchette englobant l'intégralité de l'histogramme est inférieure à $20 \mu \mathrm{m}$. Rappelons que, pour le calcaire bathonien, cette fourchette était de l'ordre de $25 \mu \mathrm{m}$ à $75 \mu \mathrm{m}$.

Cette étude qualitative, nous permet donc de déterminer avec précision les faciès de calcaires rencontrés au sein des pierres gravées de la Marche. Cependant, les données microtopographiques permettent aussi de procéder à une caractérisation quantitative. Celle-ci se base sur des calculs de paramètres standards de la topographie des microsurfaces: ils permettent en effet de représenter les caractères morphologiques au sein d'un graphique. La comparaison des faciès de roches est donc, grâce à cela, plus aisée.

Nous avons, pour ce faire, effectué 57 mesures sur des échantillons géologiques. On les a ensuite analysés au moyen des deux paramètres déjà cités, à savoir le « St » et le $« \mathrm{~Sv} »$.

« Sv » est la valeur de la plus profonde vallée de la surface (différence entre le plan moyen et la vallée la plus profonde) et « St » la valeur de la hauteur totale de la surface (différence entre le pic le plus haut et le creux le plus profond). Ainsi, «St » décrit bien l'hétérogénéité de la microtopographie d'une surface et « $\mathrm{Sv}$ » identifie la présence de creux 


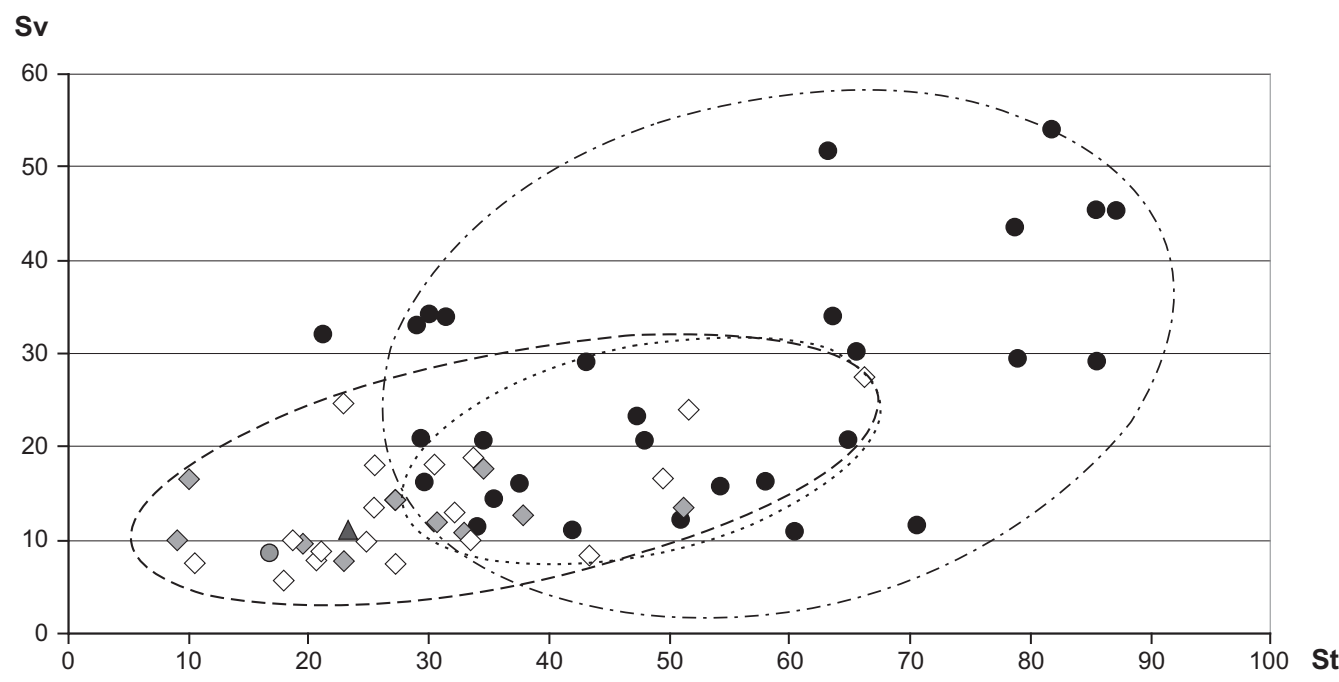

\begin{tabular}{|ccr|}
\hline Calcaire bathonien & $\diamond$ Kimméridgien champ (frais) & $\Delta$ MH-50-7-42-I \\
$\bigcirc$ Calcaire bathonien (surface lustrée) & $\diamond$ Kimméridgien affleurement & --- Kimméridgien \\
.... Zone principale de recoupement & $-\cdot-$ Majeure partie de la distribution du calcaire bathonien
\end{tabular}

Fig. 27 - Rapports «St/Sv» d'échantillons géologiques de calcaires kimméridgiens et bathoniens, et projection des valeurs obtenues sur une pierre gravée (MH 50-7-42).

importants sur celle-ci. Or, les résultats de cette analyse confirment les résultats de celles effectuées ci-dessus.

Le calcaire bathonien est hétérogène et possède des grains en général assez grossiers. De fait, ses valeurs « St » et « $\mathrm{Sv}$ » sont élevées par rapport au calcaire kimméridgien qui, lui, a un aspect plus fin et homogène.

Une projection des rapports « Sv/St» dans un graphique montre en effet deux nuages de points qui ont chacun été créés par une des deux matières lithiques mesurées. On observe ainsi que la distribution des spécimens bathoniens est nettement plus dispersée que celle du kimméridgien. De même, les « extrémités » du graphique constituent des zones de détermination précise de chaque faciès. Cependant, il existe, dans la partie comprise entre « St » $=28$ et $" \mathrm{St} »=69$, une zone de recoupement importante entre les deux types de roches. Celle-ci correspond à une aire où la comparaison des rapports « Sv/St » ne permet pas une distinction claire entre le calcaire kimméridgien et le calcaire bathonien. Il s'agit ici du faciès mixte que les statistiques avaient identifié.

Grâce à ce graphique de référence, et malgré l'aire plus incertaine mentionnée, il nous a été possible de confirmer la détermination pétrographique de certaines pierres gravées du site. Ainsi, un test de projection de valeurs obtenues sur une pièce montre que celle-ci se trouve bien dans le champ des calcaires kimméridgiens ( $\mathrm{MH}$ 50-7-42, fig. 27 et pl. 4).

Reste à attirer l'attention sur l'effet de l'usure: celle-ci affecte en effet les surfaces, leur microtopographie et par conséquent le calcul des paramètres de la topographie. Par exemple, la surface lustrée d'un calcaire bathonien érodé peut, dans le graphique, se placer dans l'aire des kimméridgiens. Il est donc avant tout nécessaire d'examiner soigneusement la pièce à mesurer pour détecter les endroits les plus épargnés de l'usure; ce n'est qu'à cette condition que les valeurs topographiques seront véritablement représentatives du matériau.

Ainsi, au terme de cette étude microtopographique concernant les matières premières, on peut dire que des arguments supplémentaires en faveur de la diversité des supports utilisés à la Marche ont été apportés. Les deux calcaires les plus présents, le bathonien et le kimméridgien, appartiennent en effet à des faciès très différents, en termes de planéité globale et de rugosité. Il est notable que les Magdaléniens n'ont pas uniquement choisi pour leurs activités (qu'elles soient artistiques ou non) le type de calcaire présent autour du site. Cela témoigne d'une attention portée aux propriétés intrinsèques des roches, 
attention que l'on s'efforcera d'expliciter, notamment pour ce qui est de la technologie de la gravure (voir infra).

\section{TECHNOLOGIE DE LA GRAVURE}

\section{INTERACTION OUTIL/SURFACE: ASPECTS MÉCANIQUES ET PHYSIQUES DE LA GRAVURE}

La force principale qui concerne l'action de graver est bien sûr celle exercée par la main du graveur sur la surface, par le biais de la partie active de l'outil. Elle est directement transmise sur le support au niveau du point de contact entre la surface gravée et l'instrument. Celui-ci correspond à la pointe de l'outil. La force exercée a donc un impact direct sur la morphologie du tracé. Nous avons réalisé des mesures quantitatives sur la relation qui existe entre la force exercée sur l'outil et la profondeur du tracé gravé (fig. 28). Ainsi peut-on en déduire un principe détaillant la relation force/ profondeur du trait qui est assez simple et s'exprime comme suit: avec une même pointe d'outil sur une même surface, l'augmentation de la force exercée provoque un creusement de plus en plus accentué de cette surface.
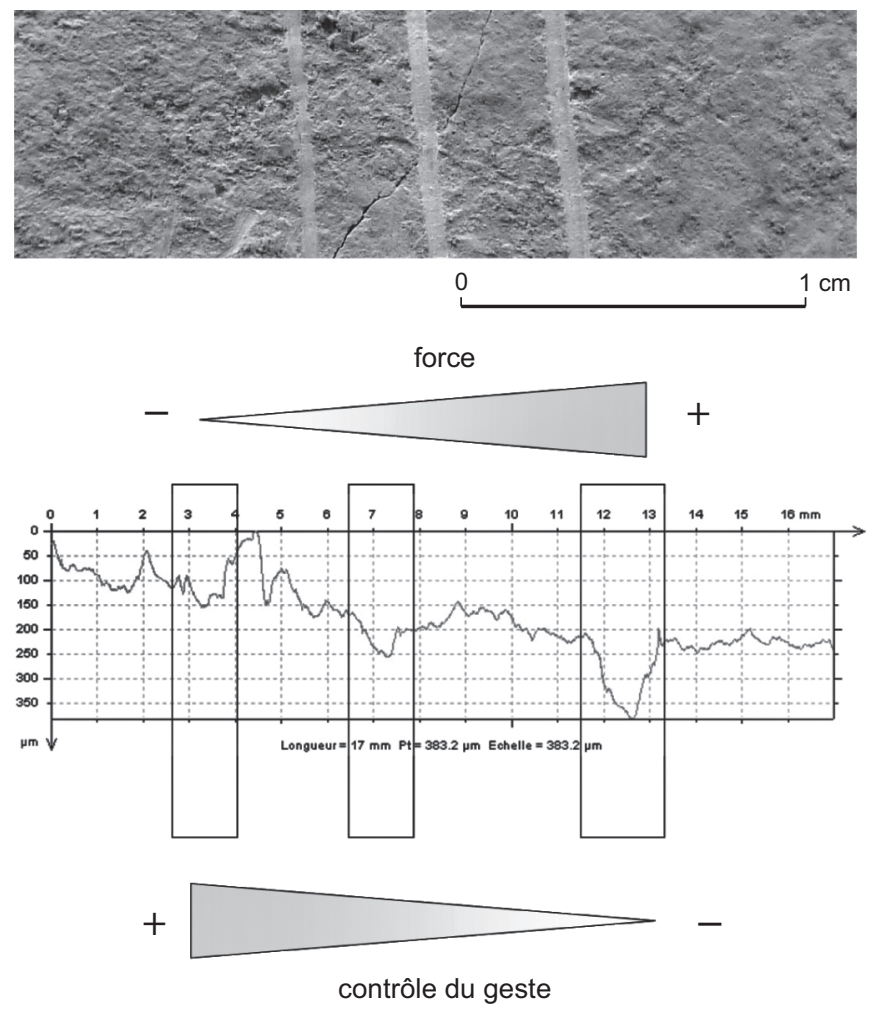

Fig. 28 - Interrelation force-profil-geste d'après un essai de gravure sur un calcaire kimméridgien (cliché : N. Mélard).
Ceci est une règle générale pour la gravure qui doit être cependant relativisée, car des matériaux différents, silex et calcaire dans notre cas, mis en contact lors d'un mouvement, se trouvent en réalité dans une interaction bilatérale qui est conditionnée par leurs propriétés mécaniques respectives. Nos expériences ont de même montré que l'augmentation de la force est limitée par les contraintes du geste nécessaire pour effectuer un trait contrôlé. C'est notamment le cas lorsque le trait est courbe et que la force d'impact doit être adaptée. Le graveur doit donc trouver un juste milieu entre force et geste pour réaliser au mieux ses gravures.

Dans notre pratique de la gravure il s'est ainsi avéré qu'il est possible de réaliser un tracé droit d'une profondeur de $300 \mu \mathrm{m}$ environ sur une surface calcaire (kimméridgien). La force nécessaire à cette action étant assez élevée, le contrôle de la course du tracé s'en est trouvé réduit. Pour graver des courbes, il nous a été nécessaire de réduire la force exercée, ce qui a eu pour conséquence des traits moins profonds. Or sur les pierres de la Marche nous avons remarqué que la profondeur d'un tracé simple ne dépasse que très rarement $150 \mu \mathrm{m}(0,15 \mathrm{~mm})$ et mesure souvent moins de $100 \mu \mathrm{m}$. Ceci montre que les graveurs magdaléniens du site ont su adapter leur geste et leur force aux contraintes des matériaux qu'ils utilisaient pour réaliser des motifs qui parfois nous surprennent par leur richesse en termes de détails.

\section{TECHNIQUES ET NOTIONS TECHNOLOGIQUES DANS L'ÉTUDE DES PIERRES DE LA MARCHE}

Ces constatations préliminaires faites, nous avons tenté d'examiner en détail les techniques employées pour la réalisation des traits gravés. Nous avons écarté, pour ce faire, certaines pierres qui ne portent pas de gravures ou sur lesquelles les gravures sont douteuses et pourraient correspondre à des traces d'utilisation. Nous avons de ce fait identifié 1645 surfaces gravées sur 1221 pierres. En effet, 424 pierres ont été gravées des deux côtés. Pour nos décomptes les surfaces ont été traitées individuellement, suivant le type de tracé qui s'y trouve (fig. 29).

Parmi l'ensemble des surfaces gravées, 998 portent de nombreux traits dont 348 de véritables accumulations de traits entrecroisés. 267 pierres ne sont gravées que de quelques rares traits et 32 supports ne possèdent qu'un seul trait gravé mais de manière telle que son identification comme gravure ne laisse aucun doute.

La plupart des gravures ont par ailleurs été réalisées sur des surfaces lisses. En effet, 939 des surfaces choisies par les graveurs sont les plus planes possibles; le choix de faces 


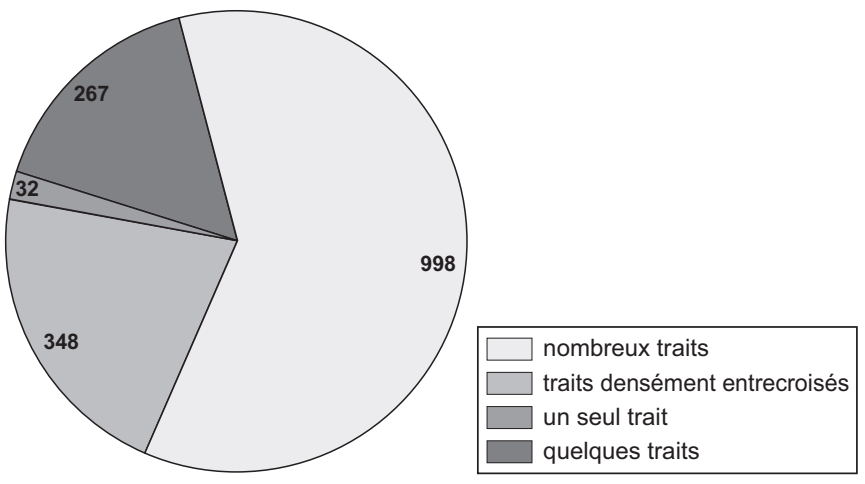

Fig. 29 - Densité des gravures sur les pierres de la Marche (1645 surfaces pour 1221 pierres, recto et verso séparés).

rugueuses et irrégulières pour graver des motifs n'a été constaté que dans 54 cas.

En ce qui concerne la morphologie des tracés, nous remarquons que les gravures fines dominent le corpus de la Marche (786 cas). Cet ensemble inclut les gravures à multiples passages. Les tracés à passage simple ont, quant à eux, été répertoriés sur 362 supports. Les tracés profonds sont beaucoup plus rares (73 cas). Les trois types de tracés peuvent apparaître ensemble sur une surface. La sculpture enfin, telle qu'elle a été remarquée dans quelques rares cas par L. Pales, n’a pas été identifiée dans notre étude.

Pour préciser les connaissances sur la nature des tracés et la technique appliquée pour les produire, nous avons réalisé des études au microrugosimètre. Ceci nous a permis de travailler de manière plus précise sur les aspects morphologiques et techniques de la gravure des pierres de la Marche. Il devient alors possible, au travers de quelques exemples, de détailler les résultats de l'étude sur la chronologie des traits, la morphologie des tracés et la reconstitution des gestes du graveur.

\section{NATURE ET EMPLOI DES TRACÉS}

Étudier en détail les gravures et notamment vouloir aborder leurs aspects techniques demande au préalable de connaître leur morphologie précise. On distingue à ce titre deux grands types de traits: les traits fins et les traits profonds.

\section{Les traits fins}

Un des caractères récurrents de la technique des graveurs de la Marche est l'emploi de traits d'une grande finesse. Or, des travaux concernant la morphologie des gravures, notamment dans les grottes ornées, et nos propres expériences ont montré que celle-ci change selon la technique de gravure mais aussi en fonction du type même du support et de ses propriétés mécaniques (Delluc, Delluc, 1983 et 1984). Les deux facteurs les plus importants sont de ce fait la dureté et la morphologie globale (rugosité, homogénéité).

Une observation précise, servie par des moyens d'investigation adaptés, permet d'appréhender ces traits dans le détail.

La plupart du temps, ceux-ci sont fins mais assez marqués pour être distingués à l'œil nu, avec une lumière rasante. Beaucoup de plaquettes de la Marche portent ce genre de tracés. Il s'agit de gravures généralement produites par un seul passage. Le profil du trait possède une forme en V. Il est cependant important de noter qu'au fond du tracé peuvent apparaître des sillons supplémentaires qui donnent un caractère particulier au profil. Sa profondeur oscille entre $20 \mu \mathrm{m}$ et $150 \mu \mathrm{m}$ selon les traits.

Les gravures les plus fines sont, quant à elles, à peine visibles à l'œil nu. Elles ont une profondeur qui oscille entre $20 \mu \mathrm{m}$ et $100 \mu \mathrm{m}$ et leur largeur dépasse rarement $200 \mu \mathrm{m}$ $(0,2 \mathrm{~mm})$.

Il semble que, dans certains cas, ces traces fines soient des témoins de gravures initialement plus profondes mais ayant subi une altération par frottement. Celui-ci peut en effet provoquer une abrasion partielle de la surface. En conséquence, le tracé paraît moins profond qu'il ne l'était initialement. Dans ce cas, nous sommes sans doute face au résultat d'une usure progressive liée à la circulation du support dans le gisement avec des phases successives de gravure et d'abandon.

Dans d'autres cas, les tracés fins semblent avoir été gravés avec soin mais peu profondément. Aucun indice ne laisse penser que leur faible profondeur est due à une abrasion par usure. Dans le contexte de leur emploi pour une représentation figurative, ces tracés se trouvent en général au-dessus des tracés principaux. Ils ont en effet souvent été gravés postérieurement à une silhouette. Leur fonction est, semble-t-il, de compléter le tracé lui-même en le prolongeant ou de parfaire le motif. Dans ce dernier cas, les traits fins sont alors employés pour représenter des détails figuratifs tels que le pelage. De même, lorsqu'on observe des figures animales et humaines, on remarque régulièrement des tracés fins proches des traits de la silhouette et parallèles à ceux-ci, souvent légèrement décalés vers l'intérieur de la figure. Un tel appui graphique donne une impression de 
volume et contribue ainsi à l'allure naturelle de la figure représentée. Un des rennes de la Marche illustre bien cette technique (MH 50-7-275, fig. 14 et pl. 7). Ici, ce sont les traits de contour du corps qui sont appuyés au moyen d'une reprise par traits fins. L'artiste a cependant choisi d'exécuter les bois de l'animal différemment. Ils sont en effet gravés par des lignes à passage simple, sans reprise: leur forme se détache ainsi nettement et apparaît plus lisse et plus dure, comme l'est cette partie du corps de l'animal. La technique de gravure employée contribue donc au naturalisme de la représentation et témoigne de la maîtrise de l'artiste.

Parfois, enfin, les gravures fines précèdent des traçages plus profonds. Un tel phénomène peut être observé lorsque le tracé subséquent et plus profond ne retrace pas l'intégralité du trait plus fin. Dans ce cas, des résidus témoignant de l'existence de ce dernier, sur le bord ou même à l'intérieur de tracé, peuvent être observés (fig. 32). Dans le cas inverse, le passage de l'outil de gravure lors de la production du tracé plus marqué efface totalement le trait précédent. Ce genre de phénomène n'est pas facile à mettre en évidence. La plupart du temps, seule l'étude des relevés microtopographiques nous montre une telle association. Il est probable que, dans de tels cas de figures, nous avons affaire à des tracés préparatoires qui ont servi à esquisser le dessin et guider le tracé définitif de la représentation. Ceci sous-entend donc que l'artiste a soigneusement préparé son dessin.

\section{Les traits profonds}

Nous rencontrons parfois à la Marche des gravures réalisées au moyen de tracés dont la profondeur dépasse les $500 \mu \mathrm{m}$ et dont la largeur est supérieure à $1 \mathrm{~mm}$. Le creusement de la surface forme de fait des sillons dont le profil est en U. Ces gravures restent cependant rares: seules quelques figurations présentent ce type de tracés. On peut citer, à titre d'exemple, la tête humaine en profil droit qui figure en premier dans l'étude de L. Pales sur les humains (Pales, Tassin de Saint-Péreuse, 1976). De même, une femme assez stylisée que nous avons identifiée sur une pierre montre l'emploi de tracés profonds pour sa réalisation (Ja 138, pl. 35). Il est par ailleurs notable que ces deux représentations sont en elles-mêmes tout à fait particulières. En effet, la tête humaine est en réalité une tête "bestialisée " et la femme se trouve sur un support peu courant, puisqu'il s'agit d'un galet.

Un autre cas est, lui aussi, éloquent quant au contexte particulier de l'emploi des traits profonds. Une pièce consti- tuée d'une roche très poreuse et tendre porte ainsi des tracés indéterminés mesurant parfois plus de $1 \mathrm{~mm}$ de profondeur. Le parcours des sillons aussi bien que la rugosité de la surface à l'intérieur du tracé (qui se distingue de la surface environnante) montrent qu'il s'agit ici de traces anthropiques. Il est de même probable que ces traits correspondent à des gravures intentionnelles et non à des traces d'utilisation: les tracés dessinent en effet des courbes; une opération de raclage aurait plutôt laissé des sillons droits. Le rapport spécifique support-type de trait est donc ici bien visible.

Enfin, la sculpture, telle qu'elle a été signalée par L. Pales et D. Vialou sur certaines pierres de la Marche, est totalement absente du corpus (pourtant important) que nous avons étudié (Vialou, 1979; Pales, Tassin de SaintPéreuse, 1989). Cela confirme l'hypothèse selon laquelle l'emploi des tracés profonds et de la sculpture est plutôt marginal dans la technique de gravure magdalénienne à la Marche.

Toutefois, le registre morphologique des gravures de la Marche est particulièrement riche et intéressant. Il est d'ailleurs tout à fait remarquable que, souvent, les plaquettes exploitent toute une «palette " de tracés différents. De même, ceux-ci semblent parfois être employés de façon variée afin de produire la représentation la plus satisfaisante possible. Le rapport entre les traits de gravure, quand il existe (et encore faut-il savoir le déterminer), ne peut donc être omis. Dans ces conditions, il paraît naturel de s'interroger également sur la chronologie des traits.

\section{CHRONOLOGIE RELATIVE DES GRAVURES}

Un motif est composé d'un ensemble de traits successivement gravés sur une surface. Lors de son élaboration, les traits se recoupent de manière plus ou moins répétée selon la complexité du motif. Une figure superposée à une autre provoque le même effet. Or, des études déjà menées au MEB sur des pierres et des os gravés ont montré que le recoupement des tracés est un indicateur pour la chronologie relative des traits. En effet, lors du passage d'un trait au-dessus d'un autre déjà existant, celui-ci laisse, au sein du sillon antérieur, un certain nombre de traces, voire une empreinte (D'Errico, 1994; Fritz, 1999).

Les études microtopographiques permettent d'abord de mettre en évidence les mêmes phénomènes que le MEB. En effet, nous remarquons, en étudiant les entrecroisements de traits, la marque du tracé postérieur sur le précédent: il s'agit souvent d'un petit sillon situé au fond ou sur les bords 
du tracé antérieur (pl. 21). Nous avons de même, à plusieurs reprises, remarqué des ébréchures sur un des deux bords du tracé déjà existant. Il semble que celles-ci se produisent à la sortie de l'outil de gravure du fond du premier tracé, lors de la réalisation du second. Les sillons et l'ébréchure peuvent être plus ou moins marqués: ceci dépend de la force appliquée pour la gravure, de la vitesse d'exécution et de la largeur des deux tracés. Cependant, l'usure peut atténuer ces traces; si celle-ci est trop importante, il arrive même que nous ne puissions plus les repérer ni établir la chronologie relative des traits.

Pourtant, la détermination de cette chronologie nous permet d'acquérir des informations précieuses sur la technique de gravure mise en œuvre et, parfois aussi, des éléments intéressants pour nourrir une réflexion sur la symbolique des motifs.

En effet nous pouvons, par le biais de la chronologie relative, établir la succession de deux représentations. Sur une pierre portant la représentation d'un loup et d'un cervidé par exemple, on observe que ce dernier a été gravé antérieurement. Ceci associé au fait que le loup est plus complet que le cervidé (dont nous ne connaissons qu'un arrière-train incomplet) nous permet de retracer «l'histoire » de cette pièce: il semble que le support ait été fracturé deux fois. Une première plaquette, bien plus grande que la pièce actuelle, fut d'abord gravée d'un cervidé, puis s'est fracturée. Le loup a ensuite été gravé sur un des fragments. Enfin, le support s'est cassé une seconde fois en emportant le bout du museau et une partie de l'arrière-train de cet animal.

L'étude de la chronologie des traits sur une pierre avec deux têtes « en Janus » (Pales, Tassin de Saint-Péreuse, 1976) nous fournit, quant à elle des renseignements sur la composition graphique d'un motif. Ainsi le graveur magdalénien a-t-il d'abord réalisé la tête de droite puis celle de gauche. Une liaison entre les deux a été créée au sein de la longue chevelure des personnages. C'est d'ailleurs cet entrecroisement des traits qui nous a permis de les analyser.

La chronologie des traits peut, enfin, nous permettre d'établir des liens logiques entre des figurations, liens qui nous serviront ultérieurement dans le cadre de l'interprétation. À cet égard, une plaquette particulièrement intéressante provient des collections Lwoff. Chaque chercheur qui a travaillé sur la Marche l'a intensivement analysée. L. Pales proposait déjà l'hypothèse selon laquelle nous serions en face de la superposition d'une femme et d'un nouveau-né (Pales, Tassin de Saint-Péreuse, 1976). La théorie du «mythe de Lussac-Angles » de J. Airvaux inclut également cette figuration (Airvaux, 2001 et 2002). C'est enfin J.-P. Mohen qui s'interroge à nouveau sur l'importance de cette pièce (Mohen, 2002). Nous avons nous-même étudié la superposition des deux figures et ses étapes de réalisation. Deux endroits montrent des éléments particulièrement intéressants pour la compréhension de la plaquette. Au niveau du nez du nouveau-né d'abord, on peut observer une telle association (pl. 1 et 3b) : les lignes de cette partie de l'anatomie se superposent nettement à celles de la poitrine de la femme. Le bébé a, en conséquence, été dessiné après elle. De même, une étude des deux lignes occipitales du nouveau-né montre que celle de droite a été tracée après celle de gauche. Or, comme L. Pales l'a remarqué pour cette pièce, le second tracé (en termes de chronologie) est " anatomiquement, le plus satisfaisant »(Pales, Tassin de SaintPéreuse, 1976). En effet, il possède des proportions plus «authentiques", pour un homme adulte. L. Pales a d'abord affirmé qu'il s'agissait ici d'un " raté » du graveur, corrigé avec la seconde ligne (Pales, Tassin de Saint-Péreuse, 1969). Quelques années plus tard, il a plutôt pensé un céphalématome (Pales, Tassin de Saint-Péreuse, 1976). Nous pensons, quant à nous, que la première hypothèse est à exclure car le graveur a insisté sur cette forme en retraçant son parcours à plusieurs reprises. De ce fait, nous sommes persuadés que les deux tracés de l'occiput sont intentionnels. Reste à appréhender le sens de cette association.

La détermination de la chronologie relative est donc, à plusieurs égards, très intéressante. Reste à noter qu'elle peut aussi se déterminer par une observation précise des états de conservation des tracés sur les surfaces. Nous avons en effet remarqué, sur un certain nombre de pierres, qu'il existe des interrelations entre des gravures et des usures. L'étude de la chronologie entre les traits usés et les tracés plus frais, ainsi que la relation entre les zones lustrées ou polies et les gravures est un indicateur pour « l'histoire » de chaque support. Pour étudier cette interrelation, il n'est pas forcément nécessaire d'avoir recours à des techniques très poussées telles que la microtopographie. Une observation détaillée sous la loupe binoculaire par exemple peut déjà apporter des éléments intéressants. Cependant, une caractérisation qualitative plus précise et une mesure exacte des impacts des usures sur les gravures et la modification des surfaces demandent le recours à des moyens d'investigation plus adaptés comme la microtopographie. Ceux-ci apportent par exemple la possibilité de comparer des types d'usure sur une même pièce mais dans des zones différentes. Nous avons procédé ainsi dans le cas du recto et du verso de la pierre qui porte les gravures d'une femme assise 
et d'une tête humaine (pl. 10 et 12). En effet, nous avons observé, d'un côté et de l'autre de celle-ci, une différence assez frappante de l'état de conservation des gravures. Le verso porte, à l'heure actuelle, quelques résidus de tracés seulement, tandis que le recto possède un grand nombre de gravures plus fraîches. Cependant, nous y trouvons également des tracés usés, notamment sur le bord de la pièce. Il semble donc qu'il y ait eu, dans cet exemple, une nouvelle opération de gravure, mais décalée dans le temps et ne concernant qu'un côté de la pierre, ou encore qu'une face ait été plus préservée que l'autre de l'altération. Ces éléments peuvent donc nourrir une future interprétation.

L'étude de la chronologie relative des gravures peut ainsi nous renseigner sur la complexité de l'histoire d'un support. Mais, pour être exhaustif, il convient également de se pencher plus précisément sur le rapport entre morphologie des tracés et détermination de l'outil de gravure.

\section{MORPHOLOGIE DES TRACÉS ET IDENTIFICATION DES OUTILS DE GRAVURE}

La microtopographie nous permet d'étudier de manière très précise la morphologie d'un tracé à des échelles différentes. On peut ainsi tenter d'appréhender quels impacts un outil de gravure peut avoir sur une surface lors de la gravure puisque, pendant celle-ci, les deux corps (outil et surface) sont mis en contact.

Au niveau des microsurfaces, nous pouvons caractériser le tracé d'un point de vue tribologique et ainsi déterminer la modification de la surface par l'outil de gravure. La comparaison entre l'intérieur d'un tracé gravé et une surface naturelle sans trace nous montre bien la différence de rugosité entre les deux (pl. 4). En effet, l'intérieur du trait possède un aspect nettement plus lisse que la surface naturelle (fig. 30). Il se rapproche morphologiquement d'une surface présentant un lustre identique à celui que nous avons mesuré sur le bord supérieur de la même pièce. Ceci montre que le passage de l'outil provoque en plus du creusement un lissage dans la zone de contact entre l'outil et la surface gravée.

Le lissage est donc un indicateur du passage d'un outil de gravure. Une aspérité ou une fissure naturelle ne possèdent pas cette morphologie et peuvent en conséquence être distinguées d'un trait gravé par la mesure microtopographique.

À une autre échelle, la microtopographie permet de s'intéresser aux profils des traits. Nous rappelons que, par ces relevés, il est possible d'extraire des profils à chaque

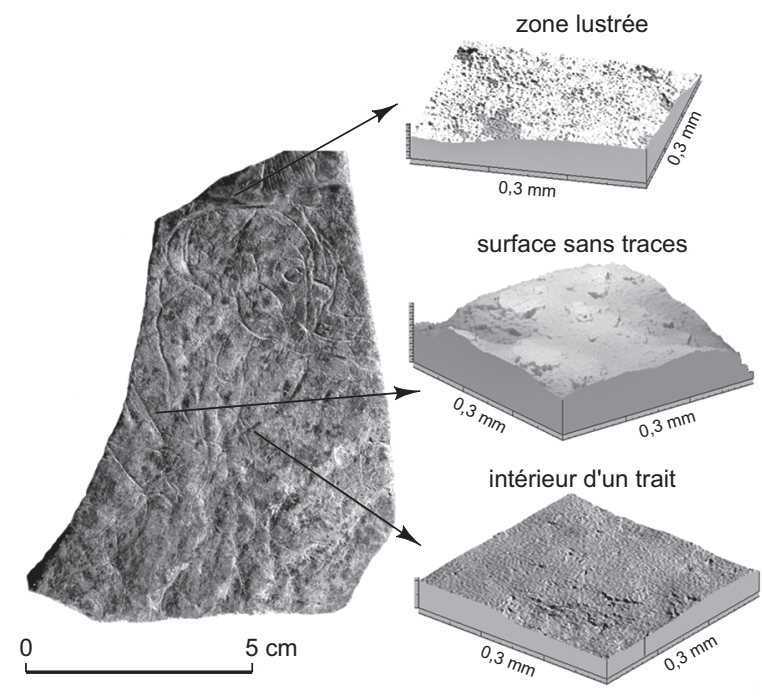

Fig. 30 - Comparaison microtopographique de trois zones (zone lustrée, surface sans trace anthropique et intérieur d'un trait) sur une pierre gravée (MH 50-7-42, voir pl. 1 à 4) (cliché: D. Bagault, Centre de recherche et de restauration des musées de France).

endroit de la surface d'un objet. Ceux-ci, qui sont en quelque sorte l'empreinte de l'outil laissé lors du ou des passages de gravure, portent en leur sein les propriétés morphologiques spécifiques à ce dernier. Il faut cependant attirer l'attention sur les problèmes liés à la caractérisation des traits gravés par leur profil et donc sur les précautions à prendre lors de l'étude. Celle-ci nous montre en effet que le profil d'un tracé peut se modifier lors de son parcours. Le choix des tracés pour déterminer le profil caractéristique d'un outil est donc difficile et il doit être fait après l'analyse détaillée de plusieurs endroits du tracé.

Si l'on a affaire à une pièce portant plusieurs motifs distinguables, il peut être possible de repérer différents outils. De ce fait, la combinaison de deux études, celle des motifs et celle concernant la morphologie des tracés, permet parfois de fournir des informations précieuses sur la relation entre des gravures superposées. Nous pouvons, à titre d'exemple, reprendre le cas du cervidé et du loup évoqué plus haut (pl. 36 à 39). Celui-ci nous permet de montrer en quoi l'analyse de la morphologie peut confirmer ou non ce qui a été dit de la chronologie relative. Les profils extraits sur les motifs semblent ainsi comporter des spécificités qui nous permettent de les caractériser (pl. 39). Leurs différences apparaissent clairement: celui du cervidé se caractérise par un tracé portant en son sein deux creux, tandis que celui du loup montre un fond moins accidenté. Les sections d'un des nombreux tracés indéterminés de la pièce possèdent 
une morphologie encore différente puisqu'un seul creux au fond du sillon est observable. Ceci montre qu'au moins trois pointes d'outil différentes ont été appliquées sur le support. De même, le fait que chacune des figures possède son propre type de trait (d'un point de vue morphologique) vient renforcer l'hypothèse selon laquelle les deux principales figures (cervidé et loup) ont été gravées avec un décalage chronologique.

L'étude du profil apporte donc beaucoup à la compréhension des traits eux-mêmes. Cependant, elle permet aussi d'envisager plus précisément l'outil et, par là même, la technique et le geste des graveurs magdaléniens: ceux-ci sont en effet liés à la spécificité de la partie active de l'outil de gravure.

\section{MIGROMORPHOLOGIES : TÉMOINS DE LA TECHNIQUE DU GRAVEUR}

Chaque gravure de trait à passage simple, que l'on observe sur une surface, est le résultat d'un geste continu sur celle-ci. Les traits à passages multiples sont, quant à eux, la somme de plusieurs gestes continus exécutés par l'homme. Ces derniers sont issus de trois paramètres majeurs qui génèrent et conditionnent la nature du trait gravé résultant de l'action de gravure: la direction du geste, la force appliquée, et la course du geste continu. Chacun de ces éléments provoque une modification de l'impact de l'outil de gravure sur la surface. L'étude des gestes qui ont produit les gravures est ainsi une opportunité intéressante pour la compréhension des techniques.

\section{Direction de la gravure}

Des recherches ont déjà été effectuées sur les microformes qui se trouvent à l'intérieur du tracé de gravure, quand celle-ci concerne l'os (D'Errico, 1988 et 1994; Fritz, 1999). Avec ce matériau, il est possible de lire les microformes à l'intérieur du tracé et de déduire, entre autres, la direction de la gravure. La pierre étant un matériau très différent de l'os, surtout en ce qui concerne ses propriétés mécaniques, nous ne pouvons pas appliquer les mêmes démarches à celle-ci. En effet, elle est plus dure et moins élastique que l'os. De ce fait, l'action de gravure provoque majoritairement un enlèvement de matière, aussi bien sur l'outil de gravure (usure) que sur la surface (incision). La déformation est, selon la roche concernée, minime voire quasiment absente. Qui plus est, la roche est plus hétérogène dans sa composition granulométrique que l'os. Les microformes que l'on peut observer dans les traits gravés sur os ne se produisent donc pas lors de la gravure sur pierre.

La direction du geste est guidée soit par un vecteur combinant un point de départ, une direction et un point final donnés (traits droits), soit par une série de vecteurs (traits courbes). Le début et la fin d'un trait sont différents en termes de morphologie, ce qui peut être étudié sur les relevés microtopographiques. Ceci est seulement valable pour les tracés simples dont les extrémités sont conservées.

Nous avons étudié le geste du graveur à plusieurs reprises lors de notre étude. On peut par exemple mentionner le cas du tracé d'un bras d'une petite figure humaine. Au niveau du coude notamment, nous avons pu montrer en quoi la composition de cet élément anatomique du personnage s'est faite de façon successive (fig. 31). En effet, le graveur a réalisé ce détail en trois mouvements: il a d'abord dessiné le bras de bas en haut, puis l'avant-bras a été tracé de bas en haut également, avec cependant le maintien d'un léger espace entre le début du trait du bras et la fin du tracé de l'avant-bras. Enfin, le graveur a relié les deux tracés par un autre, léger, qui recoupe les deux précédents.

C'est l'utilisation de la microtopographie qui nous permet l'acquisition d'informations aussi précises. En effet, comme on le voit sur l'image de synthèse, la morphologie du tracé se modifie et donne ainsi des indices précieux sur la direction du geste. D'après nos propres expériences, on peut dire qu'en général, au moment où le graveur entame la surface avec son outil, il exerce sur celui-ci une force légèrement moindre que sur le reste du tracé, afin de débuter de manière bien contrôlée sa gravure. L'augmentation progressive de la pression exercée sur l'outil se traduit par un creusement et un évasement du trait, qui se manifeste clairement sur l'image microtopographique. La fin du tracé est souvent plus abrupte que le début car le geste peut être interrompu à tout moment pendant l'acte de gravure.

\section{Course du geste}

La course d'un tracé se caractérise par la longueur du geste continu et le changement de direction du mouvement lors de son exécution.

Nous rencontrons ainsi des traits plus ou moins courts et plus ou moins courbes. Lorsqu'on observe une gravure, on s'aperçoit qu'un tracé du même type peut être exécuté de manière différente. Nous prenons à ce titre l'exemple d'une ligne ondulée avec trois changements de tracés différents. Un tel tracé peut être dessiné en une seule fois, dans un seul geste sans interruption. Le même tracé peut également 

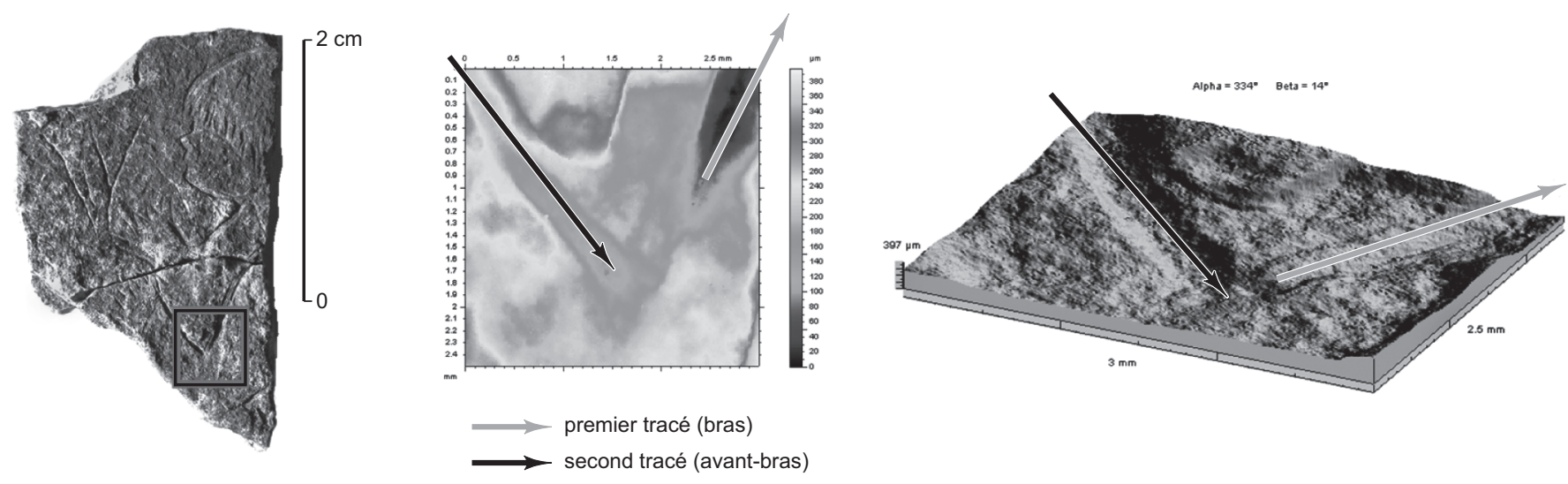

Fig. 31 - Reconstitution de la technique et des phases de réalisation du bras d’une figure humaine (Ja 711, voir pl. 28 et 29) grâce à l'analyse micromorphologique des traits gravés (cliché : N. Mélard).

être composé de multiples traits plus courts qui, dans leur ensemble, forment le même tracé que la ligne dans le premier cas. Une troisième variante consiste à reprendre une ligne dessinée dans un geste continu par des traits plus courts qui suivent la course initiale. D'autres combinaisons sont aussi possibles, telles que la répétition multiple d'un geste continu dans un seul tracé, ce qui va provoquer à terme un creusement de la surface marquant de plus en plus le tracé, pour finalement former un sillon profond.

Nous nous sommes interrogés sur les paramètres qui affectent la course d'un tracé. Les deux paramètres principaux sont, d'un côté, la nature du support et, de l'autre, le geste du graveur. Mais quel est le poids de chacun de ces paramètres en termes d'impact sur le parcours du tracé? Pour étudier cette question deux hypothèses de base peuvent être formulées, qui accordent une importance plus grande à l'un ou à l'autre des deux paramètres. Si le support est la contrainte majeure qui affecte la course du geste, sur un même matériau les courses devraient donc être assez semblables. Si l'inverse se vérifie, ce sont alors la technique et l'habitude du graveur qui jouent le rôle majeur, et on devrait trouver des traits gravés de natures variées sur un même matériau.

Il semble que, sur les faciès des roches gravées à la Marche, le comportement et l'impact des outils de gravure lors du contact avec les surfaces ne varient pas de manière significative; de même les tracés simples sont assez homogènes, bien que la dureté du support joue un rôle sur le rapport entre la force exercée sur la pointe de l'outil et la profondeur du creusement de la roche lors de la gravure. Or, alors que, d'après ces constats, nous pourrions nous attendre à une homogénéité morphologique des tracés sur les pierres, nous rencontrons au contraire à la Marche une gamme de traits variés allant du tracé simple au sillon profond. Il est donc clair que, face à des pierres aux propriétés mécaniques constantes, cette subdivision des tracés est forcément liée au geste de gravure.

Nous avons pu étudier les techniques de gravure sur plusieurs figures. Le plus souvent, ce sont les tracés à passages multiples, composés de plusieurs traits fins, qui sont employés dans la gravure. Un tel tracé se présente dans le relevé microtopographique suivant deux caractères majeurs: le premier est le creusement de la surface au cours des passages successifs. Les exemples que nous avons étudiés nous montrent que ce creusement est plutôt faible car le graveur a plutôt décalé les tracés successifs latéralement, de sorte que les traits qui en résultent se trouvent les uns à côté des autres. Le creusement peut cependant être accentué si le graveur insiste sur le retraçage d'un certain élément graphique. Le deuxième phénomène est l'élargissement du tracé final aperçu par l'observateur.

Or, la comparaison entre plusieurs figures montre un emploi varié de ces types de traits. Nous pouvons mentionner ici l'exemple d'une représentation de femme assise. Le trait de la cuisse a été réalisé avec ce geste de retraçages multiples (pl. 11). Dans le profil, nous observons de fait un ensemble de sillons correspondant à chacun des passages de l'outil mais nous notons également que la surface n'a pas été creusée de manière très significative. En effet, la profondeur de l'ensemble des tracés ne dépasse généralement pas de beaucoup la profondeur des tracés simples présents sur la même surface (fig. 32). La même technique est apparemment mise en ouvre pour la représentation du pelage du ventre d'un renne (fig. 14 et pl. 6 et 7). Le graveur a 

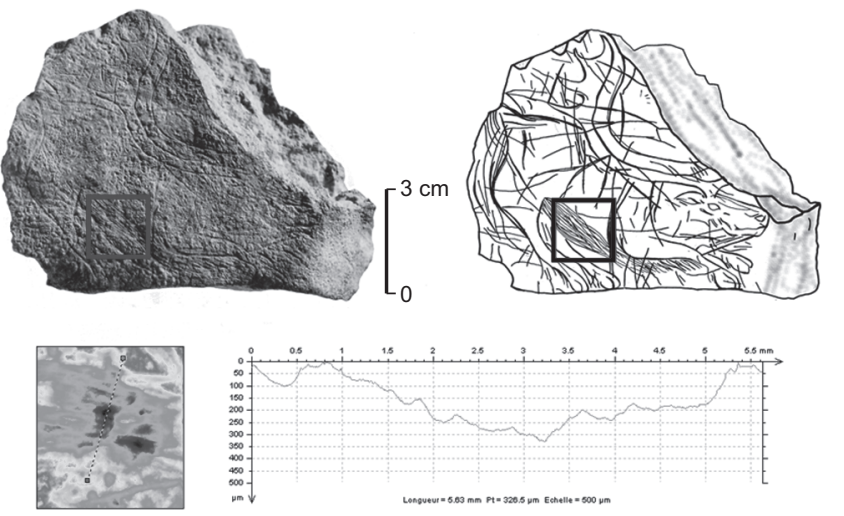

MH 50-7-275 (cf. planches 5 à 9)
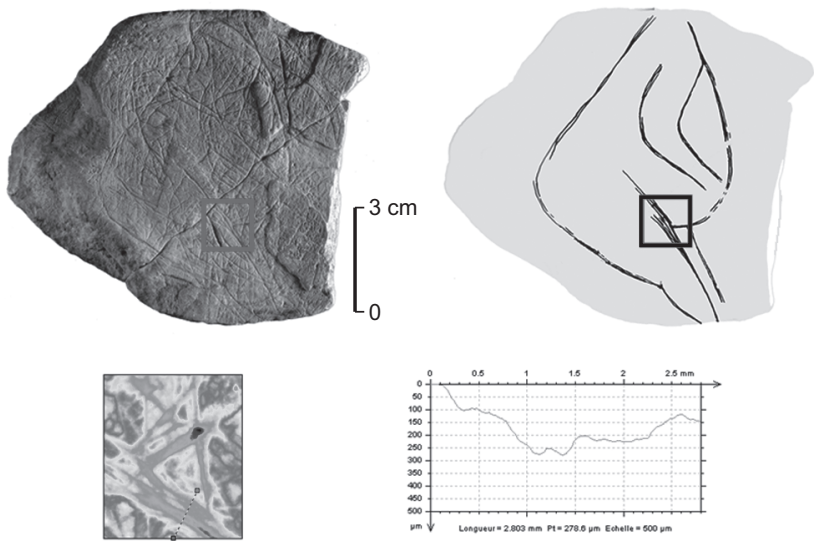

MH 50-7-276 (cf. planches 10 à 15)

Fig. 32 - Étude microtopographique de tracés à multiples passages réalisés sur deux figures de la Marche (clichés : en haut, N. Mélard; en bas, D. Bagault, Centre de recherche et de restauration des musées de France).

pris un grand soin pour représenter ce détail par une série de gravures fines et successives qui remplissent un espace limité. L'effet obtenu est, comme dans l'exemple précédent, un élargissement du tracé qui, ici, crée une zone graphique distincte par sa morphologie. Mais, à la différence de la figure de la femme assise, cette élaboration graphique du pelage ventral de l'animal a été poussée de façon à ce que le creusement de la surface soit plus important. Ce fait est observable dans le profil du tracé: en effet, un tracé simple sur cette surface mesure au maximum $120 \mu \mathrm{m}$ tandis que l'ensemble des tracés du pelage l'ont creusée jusqu'à presque $300 \mu \mathrm{m}$ de profondeur.

Le geste du graveur est donc en majeure partie issu d'un véritable choix de la part de celui-ci: si on ne peut nier le
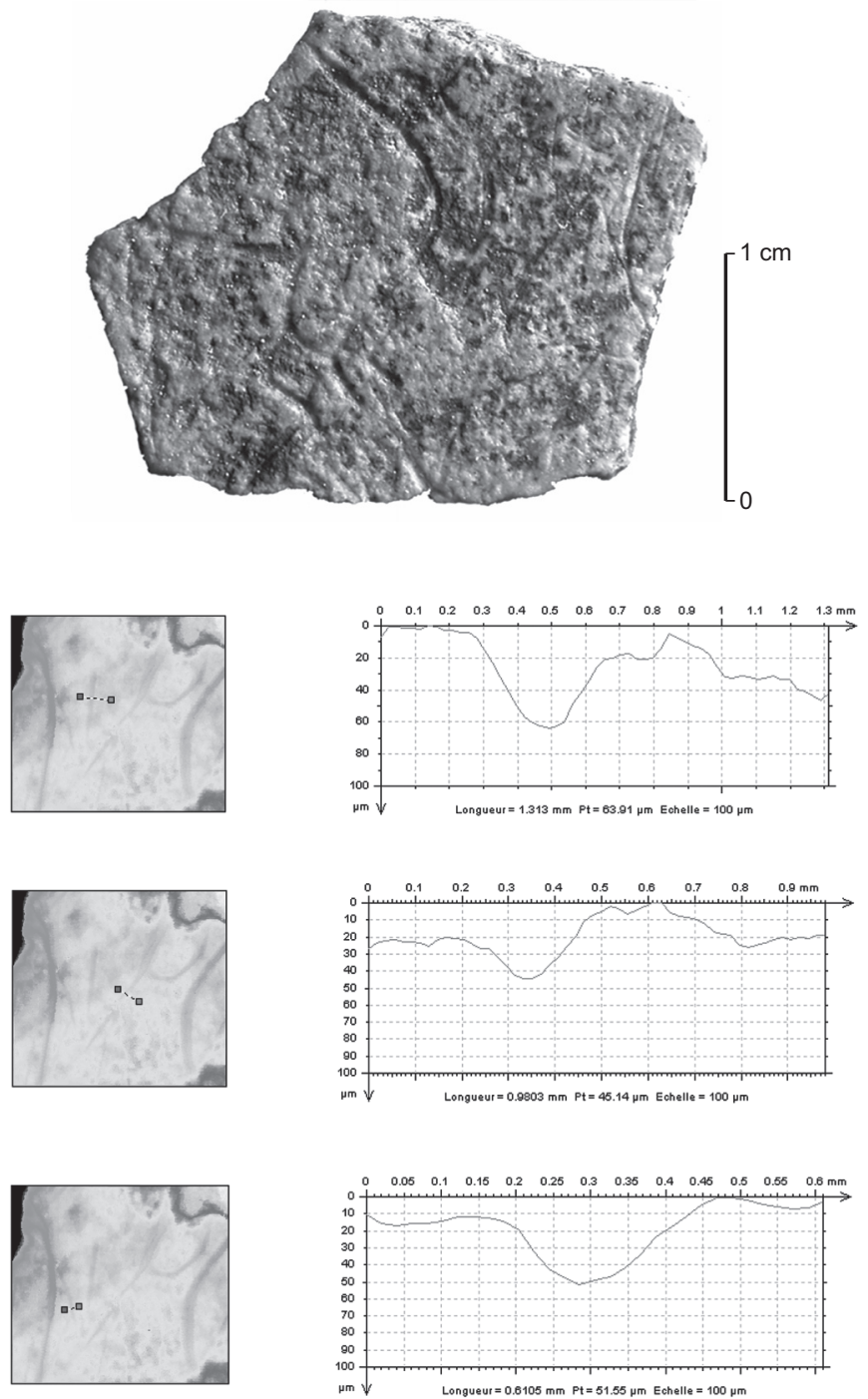

Fig. 33 - Étude microtopographique du changement de profil en fonction du changement du geste et de l'angle.

rôle du support et notamment de ces propriétés morphologiques et mécaniques dans l'opération de gravure, nous pouvons constater que celui-ci reste malgré tout secondaire.

Reste à noter que l'observation des profils permet aussi d'étudier l'angle d'attaque de l'outil de gravure et ses variations: les mouvements que les graveurs ont effectués lors de la gravure peuvent ainsi être restitués. Pour certaines figures il nous a été possible de mettre en évidence le fait que le graveur a changé l'angle selon lequel il guidait l'outil. Dans le cas d'une des petites vulves dessinées de la Marche, une comparaison entre les trois tracés formant le motif montre à la fois le changement de l'angle d'attaque 
de l'outil et le retraçage afin de marquer davantage les traits (fig. 33). Ceci démontre ainsi la « souplesse du poignet » et le soin avec lequel le graveur a su réaliser ses dessins.

Notre étude technologique montre donc que les graveurs tenaient compte des propriétés physiques des supports utilisés aussi bien que des outils de gravure. Cette attention s'exprime au travers de l'élaboration d'une technique de gravure spécifique qui se compose de la variation du geste (direction, répétition des passages) et de la force exercée sur la surface à graver.

\section{LES TRACES D’UTILISATION}

Bien sûr, l'étude des gravures est un aspect important de ce travail et celles-ci sont une des raisons pour lesquelles des plaques et plaquettes calcaires, parfois de taille importante, ont été apportées sur le site. Mais les pierres de la Marche ne peuvent être simplement définies comme des supports de gravure. Elles sont en elles-mêmes des objets archéologiques qui témoignent d'actions anthropiques autres qu'artistiques. Elles présentent en effet des traces d'utilisation que l'on observe souvent sur les plaquettes, comme des polis et frottis dus à l'usure ou bien encore des rayures ou éraflures isolées sur quelques pièces.

Leur distinction n'est pas forcément aisée: il est parfois difficile de trancher nettement entre de simples traits gravés, courts et isolés, trouvés sur 88 pierres $(6,3 \%$ du total des pierres étudiées), et des traces d'usure (fig. 34). Cependant, étant donné la nature calcaire des supports à la Marche, un simple trait fin et court, plus ou moins isolé sur la pierre, a de fortes chances d'être une trace d'utilisation. En effet, de telles incisions peuvent être produites par

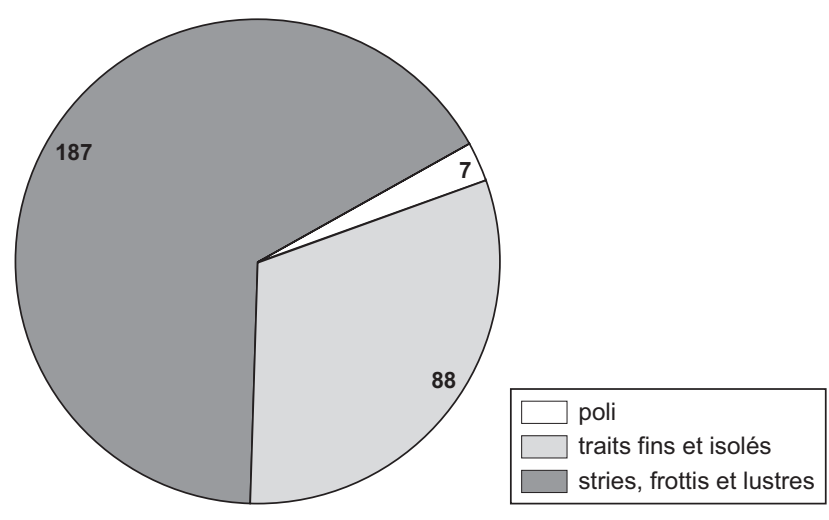

Fig. 34 - Types et fréquence des traces d'usure sur les pierres de la Marche (sur un total de 1409 pierres). chaque impact aléatoire d'une pointe assez dure et abrasive sur la surface rocheuse.

La catégorie des stries et des éraflures plus marquées est, quant à elle, plus facile à distinguer des gravures. Il s'agit de groupements de petits sillons parallèles qui ont été sans doute produits par le frottement de la surface au moyen de la partie active assez large d'un objet solide qui a été déplacé dessus. Sur plus de $13 \%$ des pierres de la Marche (soit 187 objets), nous observons ce type de tracé. Dans un cas, ces stries sont sous-jacentes à des gravures; elles ont peut-être été produites lors d'une préparation du support pour cette opération (LP 22, pl. 18 à 21 et 27). Cependant, la plupart du temps nous n'observons pas de lien direct entre les figurations et ces stries.

Un autre type de traces, encore plus nettement distinguable par rapport aux gravures, est celui de l'usure (MH 50-7-276, pl. 13 à 15). Elle se manifeste par des zones légèrement lissées qui peuvent, dans un stade plus poussé, donner lieu à de véritables polis comme nous l'avons remarqué dans sept cas.

Nous voyons donc que le nombre de traces reste assez faible par rapport au nombre des pièces étudiées. Rappelons cependant que notre étude n'a pas inclus les supports de tailles plus grandes issus des collections anciennes. Or, ceux-ci auraient pu davantage servir de base de travail que les supports de tailles réduites dont est en majeure partie constitué l'échantillon que nous avons étudié.

Une étude détaillée des types de traces propres à un ensemble d'objets s'est révélée nécessaire. Nous avons pour cela procédé à une étude morphologique de l'ensemble de celles-ci. Une grande attention a été apportée à la relation qui peut potentiellement exister entre les traces d'usure anthropiques et les gravures.

\section{LA RUBÉFACTION}

Il est par ailleurs notable que ces traces d'utilisation, tout importantes qu'elles soient, ne sont pas les seules preuves de l'utilisation anthropique des supports à des fins autres qu'artistiques: il convient en effet de mentionner que des impacts dus au feu ont aussi été découverts sur certaines pièces.

Sur l'ensemble des calcaires de la Marche, plusieurs pierres présentent des traces de rubéfaction qui pourraient être liées à des chocs thermiques. S. Lwoff a d'ailleurs décrit dans ses travaux au moins deux foyers en place dans les couches archéologiques, dont un de forme légèrement rectangulaire. Dans ce dernier, la pierre avait apparemment 
été fortement altérée par l'action du feu (Lwoff, 1940 et 1941). Les fouilleurs ont également signalé des traces de chauffe, sans les décrire de manière plus précise.

Ces indices nous sont cependant très précieux, notamment pour comparer ce que ces chercheurs ont trouvé dans le site et ce que nous-mêmes avons observé, en termes de coloration, sur les pierres de la Marche analysées dans le cadre de cette étude.

Pour ce faire, nous avons réalisé dans un premier temps des essais de chauffe sur des blocs calcaires provenant des environs du site: cette opération avait pour but d'évaluer la nature des traces que cela peut laisser sur la roche ainsi que le changement de coloration entraîné par la chaleur. Après une chauffe de plusieurs heures dans un foyer ouvert équipé d'un cerclage de pierres calcaires, nous avons observé ces dernières et avons pu dégager trois éléments majeurs.

Les zones très noires correspondent aux cendres et à la suie, qui s'enlèvent aisément par un frottement ou par un rinçage.

En revanche, dans certains endroits la roche a changé de coloration et est devenue plus grise. Cette coloration ne s'enlève pas comme le noir que nous venons de mentionner (fig. 35).

Nous trouvons enfin, sur les roches brûlées, des endroits réellement rubéfiés et aussi résistants que cette teinte grisâtre. La roche est ainsi teintée de rouge, parfois de marron.

Ces trois phénomènes, provoqués par la chauffe dans un feu ouvert, sont donc de bons indicateurs de la présence d'un foyer. De même, grâce à eux, nous avons pu déterminer des traces de chauffe sur certaines pierres de notre corpus.

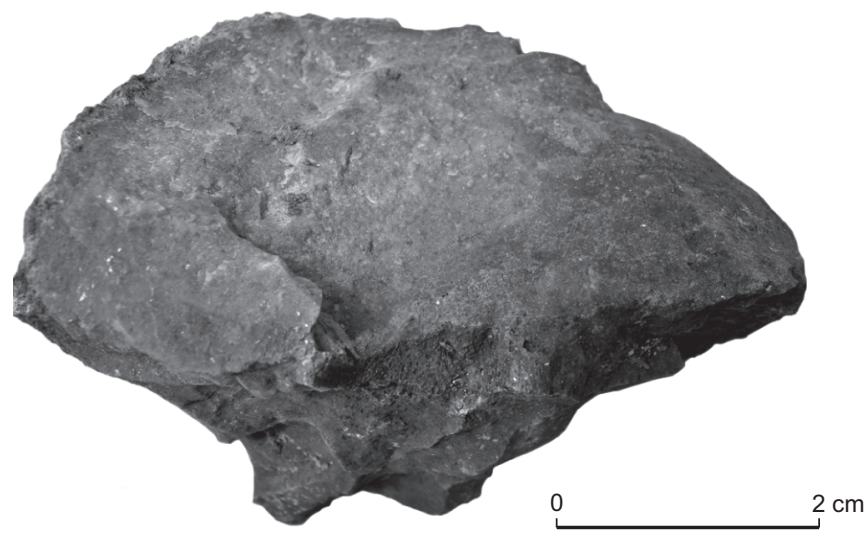

Fig. 35 - Pierre bathonienne chauffée dans un foyer ouvert pendant 8 heures puis refroidie lentement (essai et cliché: $N$. Mélard).
Deux pièces en effet montrent une forte rubéfaction: dans ces cas, l'impact du feu semble certain. 228 autres comportent également des traces qui pourraient évoquer une chauffe partielle des supports. Il s'agit de zones où la roche est teintée de gris ou comporte des taches rougeâtres qui s'intègrent dans la matière rocheuse. L'observation sous la loupe binoculaire indique qu'il ne s'agit pas de pigments : ces derniers seraient en effet appliqués sur la surface.

\section{LA FRACTURATION ET LES PROBLÈMES DE REMONTAGE}

Si la chauffe comme les traces d'utilisation témoignent clairement d'actions anthropiques, on peut cependant s'interroger sur le cas de la fracturation.

Il est de fait notable qu'à La Marche le taux de fracturation dans l'ensemble des pierres gravées est très important (Pales, Tassin de Saint-Péreuse, 1969). Il faut cependant ajouter que nous qualifions d'incomplet un support dont certains traits gravés sont visiblement interrompus sur le bord par une cassure.

Le décompte des supports incomplets par fracturation est considérable: il s'élève en effet à 588 pierres, c'est-à-dire $48 \%$ du corpus étudié. Comment expliquer ces chiffres? Il faut pour cela rappeler que la plupart des supports possèdent de petites dimensions et que leur épaisseur n'est pas très importante. Il est évident que des supports petits et fins sont plus fragiles et ont de ce fait tendance à se fracturer assez rapidement avant et lors de leur enfouissement. Pour autant, cette explication n'est pas intégralement satisfaisante. Certaines pièces plus imposantes montrent en effet des traces de fracturation. Ainsi, 169 supports fracturés, c'est-à-dire $14 \%$ du corpus, ont une épaisseur de plus de $3 \mathrm{~cm}$. On peut certes penser qu'une partie d'entre eux a subi une simple altération mécanique: les variations thermiques peuvent en effet être à l'origine de la fracturation du calcaire. Cependant, ce chiffre paraît trop important pour que cette explication soit la seule valable.

Il semble donc qu'il faille approfondir ce problème de fracturation. Il nous faudra en effet déterminer, grâce à des moyens adaptés, s'il n'y a pas eu fracturation anthropique.

Mais la fracturation est aussi une occasion d'aborder le problème du faible taux de remontage, dont nous voudrions dire ici quelques mots. Celui que nous avons pu effectuer contraste en effet avec les travaux de L. Pales qui, lui, a su remonter une centaine de pièces. Nous n'avons au contraire remonté que deux fragments. Différentes raisons peuvent être prises en considération pour expliquer ce fait. 
Nous sommes évidemment conscients que le rassemblement de pièces provenant de différentes collections que L. Pales a pu faire ne nous a pas été possible. De plus, le temps limité que nous avons passé sur cet échantillon de taille considérable ne suffit pas pour entreprendre un remontage systématique. Nous avons cependant testé des remontages ciblés, avec des supports très ressemblants en termes de dimensions et de matière première. Or, on peut noter que même des pièces qui présentaient une morphologie presque identique ne pouvaient pas être recollées. Il est de même possible que certains fragments demeurent encore sur le terrain ou dans ses environs.

Ainsi, même si un travail de remontage est indispensable à l'avenir, nous sommes convaincus que nous sommes en face d'un manque de pièces. Comme cela a été fait pour la grotte d'Enlène, à Monstesquieu-Avantès en Ariège (Clottes, 1999), nous pouvons dire que si les pièces se trouvent encore sur le site, elles ont été tellement dispersées qu'elles ne se trouvent plus dans la zone qui a été fouillée par S. Lwoff et L. Péricard dans les années 1930-1940. J. Airvaux a en effet tamisé l'intégralité des déblais de fouille (Airvaux, 2001). Il semble donc que l'on puisse être sûr qu'il ne reste plus de pierres inconnues à l'intérieur de l'abri. Même si, à l'extérieur de celui-ci, des endroits enfermant des pierres gravées existent encore, l'impact anthropique à l'origine de cette dispersion des fragments est très probable.

\section{LES TRACES D'UTILISATION ET LEURS RELATIONS AVEC LES GRAVURES}

Les moyens techniques mis à notre disposition ne nous permettent pas seulement de quantifier mais aussi de caractériser les traces d'utilisation présentes sur les pierres gravées de la Marche.

Pour plus de clarté, nous allons présenter les traces rencontrées dans un classement en deux parties. La première concerne les traces que nous appelons directes. Celles-ci sont constituées de l'ensemble des traces macroscopiques qui se trouvent sur les surfaces mais ne sont pas des gravures. Or, il s'avère que ces traces sont anthropiques. Le deuxième groupe est formé par des traces nommées indirectes. Il s'agit d'éléments d'une dégradation, elle aussi d'origine anthropique, intégrale ou partielle, et touchant des gravures ou des zones limitées des surfaces.

Les deux groupes ont fait l'objet d'une étude approfondie.

\section{LES TRACES DIRECTES}

Les traces directes individualisées sont rares à la Marche. Il semble en effet que la plupart d'entre elles, qui apparaissent sous forme de stries marquées, soient modernes: ces dernières ont souvent un aspect frais, caractérisé par la présence de poudre blanche (résidu de calcaire pulvérisé par le passage de l'outil) dans les sillons. De plus, ces traces ne semblent avoir subi aucune dégradation mécanique ou chimique et elles apparaissent sous forme de petits coups, parfois de griffures plutôt courtes. Il pourrait dès lors s'agir de coups de truelle ou d'éraflures provoquées par le frottement du tamisage pendant les travaux de terrain.

Cependant, certaines traces peuvent sans doute être qualifiées d'anciennes. Des traits courts et isolés (éraflures, rayures) sont manifestement anciens et pourraient provenir de l'impact d'un outil lors d'une utilisation quotidienne. De même, on observe des témoins nets de coups de percussion. Le calcaire, surtout quand il est homogène, répond à des chocs violents de percussion par un comportement systématique et régulier. Bien sûr, l'hétérogénéité du calcaire bathonien constitue ici un frein à l'analyse. Toutefois, quelques pièces témoignent sûrement de cette action. Ainsi disposons-nous d'une pierre gravée qui est clairement un éclat obtenu par percussion. Seul le recto est gravé et correspond à l'ancienne surface de la pierre; en revanche le verso ne possède aucun trait. Nous distinguons également le point d'impact qui a été à l'origine de l'enlèvement de cet éclat d'une surface gravée anciennement plus grande. Au total, 29 de ces éclats ont été identifiés parmi les pierres de la Marche (fig. 36).

S'il existe des éclats, il semble évident que des négatifs se trouvent aussi sur les supports de dimensions plus importantes. Effectivement, nous avons pu mettre en évidence la présence d'encoches provenant sans doute de percussions ciblées sur leurs bords. Sur les 169 fragments qui ont une épaisseur supérieure à $3 \mathrm{~cm}, 90$ portent des stigmates d'impact. Un support assez épais portant la gravure d'une vulve, par exemple, montre une encoche assez régulière (Ja 997, pl. 31). Une autre pièce montre des impacts comparables: il s'agit d'un galet gravé d'une silhouette de femme et qui montre des négatifs d'enlèvements successifs (Ja 138, pl. 34 et 35). Tout le bord gauche semble en effet être véritablement taillé.

Sur quelques pierres, nous trouvons également des zones d'écrasement qui ont la forme de creux sur la surface; celle-ci possède alors un aspect plus irrégulier. La pierre sphérique portant quelques gravures, notamment deux 

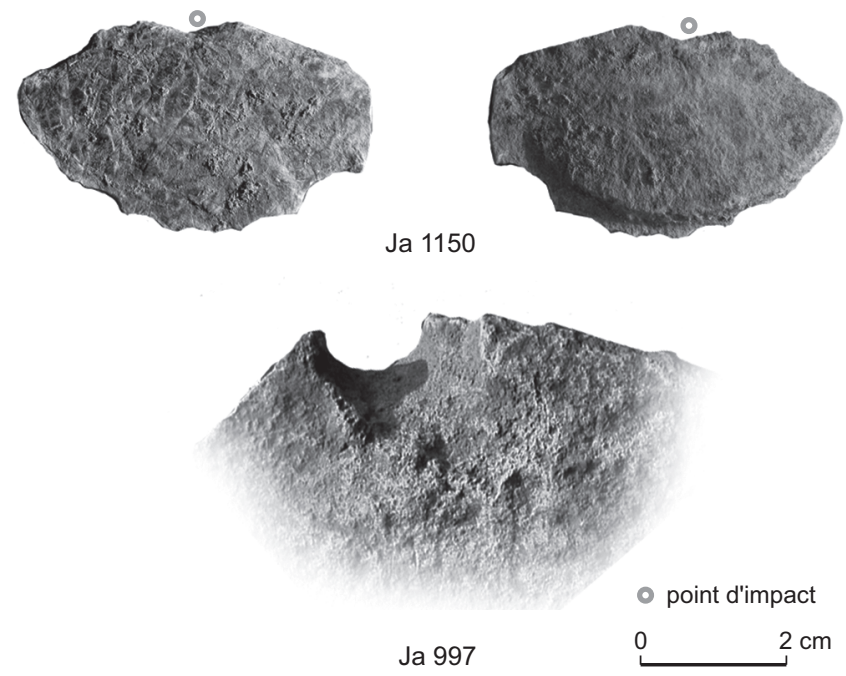

Fig. 36 - Traces de percussion sur les pierres de la Marche : éclat de calcaire avec gravures (Ja 1150) et négatif d'enlèvement sur le bord d'une pierre gravée (Ja 997, voir pl. 30 et 31) (clichés : N. Mélard).

yeux isolés, et un certain nombre de traces d'utilisation (Ja 484, pl. 42 et 44) en est une bonne illustration. En effet, à un certain endroit, l'image microtopographique met en évidence un creux de $200 \mu \mathrm{m}$ de profondeur; celui-ci comporte une surface nettement plus accidentée que le reste de la pièce. Deux origines peuvent être envisagées pour expliquer cet état de surface. Il est d'abord possible que ce soit la pierre qui, dans son intégralité, possédait un tel aspect; celle-ci aurait, par la suite, été usée. Dans ce cas, la zone serait un témoin de ce que fut auparavant la pierre, témoin préservé du fait de sa morphologie en creux. Ceci paraît cependant improbable car les zones de la pierre morphologiquement identiques à celle-ci (c'est-à-dire disposant d'une forme concave) ne possèdent pas ce type de microrelief. Une seconde explication est de ce fait envisageable: le support aurait pu en effet subir une action mécanique; il s'agirait alors d'une percussion exercée sur une autre pierre. Cette hypothèse paraît plus probable, en ceci qu'elle explique mieux l'extension limitée de ce phénomène sur la surface.

Ces témoins directs d'une action anthropique préhistorique sur les pierres de la Marche sont souvent combinés à d'autres, plus indirects.

\section{LES TRACES INDIRECTES}

L'usure est un agent majeur parmi les témoins d'utilisation ou de dégradation des plaquettes de la Marche. L'étude des gravures nous a déjà montré que certains traits étaient émoussés, dégradés ou effacés. À l'œil nu, ce phénomène se manifeste par l'augmentation de la difficulté de lecture des supports. Certains traits sont partiellement effacés. Nous avons procédé à l'étude plus approfondie de ces témoins de dégradation des surfaces. Les caractérisations microtopographiques nous permettent d'aborder ce thème de manière plus détaillée à travers quelques exemples de l'échantillon que nous avons étudié.

Ainsi, certaines pièces témoignent d'une action abrasive plus ou moins fine qui a agi sur la surface. Ce sont essentiellement des zones de lustre ou de polis. On peut citer, à titre d'exemple, la pierre qui porte la figuration d'une femme assise, découverte par L. Pales, et celle d'une tête humaine, mise en évidence par nos propres travaux (Pales, Tassin de Saint-Péreuse, 1976, fig. 17 et 18). Cette dernière comporte, sur le bord du recto, des zones très lisses et un peu luisantes. Les mesures microtopographiques ont montré que ces surfaces possèdent une autre micromorphologie que celles qui les environnent et qui sont, notamment, plus au centre de la pièce (pl. 10 à 15). Le verso est lui-même intégralement usé.

Cette pierre montre donc que les usures peuvent, sur une seule et même pierre, être différentes en termes de localisation et d'extension. De fait, les zones du recto de la pierre, que nous venons d'évoquer, peuvent être considérées comme des témoins de manipulation des supports. En effet, cette opération, si elle est répétée, finit par créer un tel lustre. Le verso, lui, nous montre un autre cas de figure: toute la surface est usée et les gravures qui s'y trouvent ne sont que des résidus.

Il est donc notable que l'usure a un impact caractéristique sur les gravures: deux phénomènes morphologiques peuvent être alors distingués. D'abord, la profondeur des traits est plus faible que pour ceux qui sont « frais » (c'està-dire qui viennent d'être tracés). De même, les bords sont émoussés et le profil du trait paraît en soi plus large.

Ce phénomène apparaît très nettement sur la pierre que nous venons d'évoquer (pl. 15). Un groupement de traits parallèles semble ainsi avoir subi une usure qui a partiellement entraîné une modification de la morphologie du tracé. En comparant deux profils, nous observons que, sur l'un des deux, les bords sont arrondis sans que la profondeur du tracé ait été réduite. Il y a donc bien eu usure, mais pas suffisamment pour que le trait de gravure soit très altéré. En revanche, aux endroits lustrés que nous avons déterminés, on peut observer des traits véritablement effacés par l'abrasion fine de la surface. Seules de légères 
traces (40 $\mu \mathrm{m}$ au maximum) ont persisté mais celles-ci ne sont plus visibles à l'œil nu; c'est le microrugosimètre qui les a révélées. Enfin, sur le verso de la même pièce, les bords des traits sont tellement usés que ceux-ci disparaissent complètement. Ceci ne se fait cependant pas de manière abrupte: les traits, en réalité, se fondent dans la surface (pl. 13 et 14a et b). En revanche, ces mêmes traits, usés à certains endroits, possèdent à d'autres des profils comportant une profondeur assez conséquente $(100 \mu \mathrm{m})$ et des bords nets (pl. 14c et d). Ainsi, même sur une surface qui paraît à première vue complètement " dégradée ", des différences en termes d'usure peuvent s'observer. Pour les distinguer, il convient néanmoins de disposer de moyens techniques performants.

$\mathrm{Au}$ final, on peut affirmer que les pierres de la Marche comportent de multiples traces d'utilisation. Il est intéressant de noter que celles-ci sont très diversifiées: d'une part on peut relever des traces directes et indirectes, d'autre part, au sein même de cette seconde catégorie, des nuances peuvent s'observer. L'usure semble en effet affecter les surfaces et, de ce fait, les gravures de manière contrastée. Cette étude nous fournit donc des pistes de réflexion intéressantes pour l'interprétation.

Mais à ces traces d'utilisation semblent s'ajouter, outre les gravures, des pigments diversifiés sur les objets de notre étude.

\section{EMPLOI DE PIGMENTS}

Un grand nombre de pigments a en effet été retrouvé dans les déblais de fouilles lors des travaux de J. Airvaux. Ils se présentaient sous des formes diverses. Selon J. Airvaux, une grande partie de la couche archéologique possédait une nuance rouge qui résulte d'un apport anthropique (Airvaux, 2001). En effet, les roches avoisinantes ne présentent pas de minéralisations frappantes d'oxydes de fer. Mais, de manière bien plus évidente, ces matières colorantes se manifestent également sous la forme de petits blocs et galets rouges. De plus, certains d'entre eux portent des traces visibles à l'œil nu et présentent une forme globale qui montre des faces d'abrasion telles qu'on les observe sur des crayons et des blocs utilisés pour produire de la poudre de matière colorante qui sert par la suite de pigment. Pour ces derniers, au moins, l'action anthropique est certaine.

Lors de l'étude des surfaces des plaquettes calcaires, nous avons observé à plusieurs reprises la présence de concentration de rouge ou l'existence de pierres intégralement rougies. Certaines portent une épaisse couche de matière. Pour étudier cette multitude de vestiges liés à l'utilisation de pigments, nous avons tenté une approche selon trois démarches.

Après avoir répertorié ces traces, notre premier but était de comprendre quels types de pigments on peut rencontrer. Pour y parvenir, nous avons procédé à un échantillonnage ciblé de 20 pierres représentatives. Au total, 45 prélèvements ont été effectués. Ces derniers ont ensuite été étudiés au moyen du MEB.

La microtopographie a ensuite été appliquée à l'étude des microsurfaces sur des grains de pigments assez grands pour être isolés et placés sous le dispositif de relevé tridimensionnel (pl. 45 et 46).

Par ailleurs, nous avons étudié le mode d'utilisation des pigments. Nous nous sommes particulièrement interrogés sur la relation qui peut exister entre les traits gravés et ces pigments.

\section{LES TEINTES PRÉSENTES SUR LES PIERRES}

Nous nous sommes efforcés dans un premier temps de classer statistiquement les supports colorés de notre corpus.

Face à notre méconnaissance des traitements postfouille subis par les pièces conservées au musée des Antiquités nationales, au musée de l'Homme et dans les collections anciennes du musée Sainte-Croix à Poitiers, nous pensons que les indications que nous allons donner sur les pigments rencontrés sont forcément incomplètes. En effet, un lavage trop intense ainsi que des prises d'empreintes par des moyens trop agressifs ont pu emporter des pigments initialement présents sur les surfaces. Malgré cela, nous avons trouvé, même sur les pierres des collections anciennes, des restes de pigments qui montrent que les pièces provenant de collections différentes sont tout à fait associables les unes aux autres.

Toutefois, par mesure de précaution, nous avons décidé de ne faire les analyses statistiques et surtout les caractérisations physico-chimiques au MEB que sur les échantillons de l'ensemble retrouvé dans les déblais de fouilles pendant la période 1989-1993.

Les décomptes ont donné les résultats suivants: pour 47 pierres, on ne peut pas clairement trancher entre l'hypothèse de traces de pigments et celle de témoins d'altération du support, soit par chauffe, soit par altération chimique naturelle; pour 175 pierres, en revanche, il semble que nous soyons en présence de pigments réels. Ce premier constat a pu être dressé grâce à nos seules observations macroscopiques et sous loupe binoculaire. 


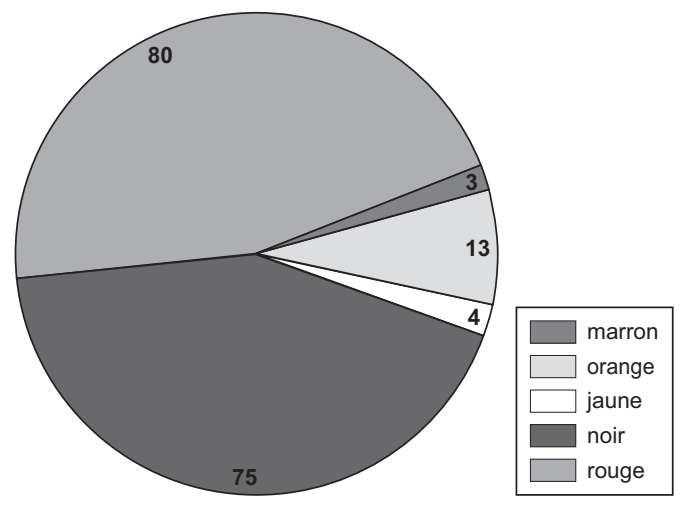

Fig. 37 - Présence de matières colorantes sur les pierres de la Marche.

Trois teintes peuvent par ailleurs être distinguées: le noir, le rouge et le jaune. La teinte rouge peut apparaître avec des nuances allant vers le marron (3 cas) ou vers l'orange (13 cas). Cependant, 80 pierres portent des traces réellement rouges et cette couleur est d'ailleurs la plus représentée. Le noir est présent sur 75 pierres. Le jaune, troisième et dernière teinte, est quant à lui plutôt rare: il n'a été relevé que dans 4 cas (fig. 37).

Les pigments, nombreux, se présentent cependant sous des formes et des quantités très diverses selon les supports. La plupart des pierres (161 pièces) montrent en effet des pigments qui ont la forme de taches plus ou moins grandes. Seules 8 pierres portent des aplats de couleur plus étalés et 6 montrent un encroûtement épais.

Nous avons également retenu, pour cette étude statistique, le type de relation qui peut exister entre gravures et pigments: 22 pierres portent uniquement des traces de pigments sans aucune gravure; 105 possèdent à la fois des gravures et des traces de pigments. Nous n'avons pas forcément pu établir de relation graphique directe entre les motifs gravés et ces pigments (motifs peints par exemple). Pourtant, 7 cas ont été trouvés, où les gravures sont soit accentuées, soit cachées par l'emploi de ceux-ci. Apparemment, pour ce qui est de ces 7 cas, ils ont été utilisés de façon à être liés avec la gravure.

L'emploi du MEB nous a permis d'effectuer une analyse physico-chimique sur ces pigments.

\section{ÉTUDE PHYSICO-CHIMIQUE (MEB)}

Malgré l'absence de motifs dessinés ou peints sur les pierres colorées à la Marche, la question qui a guidé notre démarche pour une étude plus poussée a d'abord été celle de la provenance des pigments. Nous avons pour ce faire retenu deux hypothèses plausibles, afin de confirmer ou d'infirmer nos observations macroscopiques. Celles-ci sont les suivantes: soit les pigments ont bien été appliqués par l'homme, soit ceux-ci sont contenus dans le sédiment et se sont incrustés sur les surfaces des pierres gravées.

Cette dernière hypothèse pouvait en effet être justifiée par le fait que les sédiments mêmes de la Marche sont très rouges. En revanche, l'étude de J. Airvaux en 2001 attribuait déjà une origine anthropique à certains des pigments trouvés sur le site (Airvaux, 2001).

Pour approfondir cette question nous avons donc décidé de procéder à une analyse au MEB. Cette analyse, surtout spectrométrique, a permis de caractériser de manière plus précise les pigments. Au total, nous avons échantillonné 15 pierres. Dans certains cas, différents endroits ont été échantillonnés, notamment lorsque nous avons identifié plusieurs teintes. Ce sont donc au final 21 échantillons qui ont été étudiés. Cela a donné lieu à la réalisation de 45 spectres qui nous renseignent sur la composition chimique des pigments.

Outre cette étude des pigments, une analyse des sédiments de la couche archéologique a été réalisée afin de comparer les compositions chimiques et de valider l'une ou l'autre hypothèse.

Nous commencerons donc par une présentation de la composition des sédiments de la Marche pour ensuite caractériser les pigments prélevés sur les pierres.

\section{ANALYSE DES SÉDIMENTS DE LA MARCHE}

Les sédiments analysés ici proviennent de la couche archéologique de la Marche dans laquelle ont été trouvées les pierres gravées.

Ils sont d'une teinte jaune rougeâtre et sont composés d'un mélange de matières d'origine naturelle, par le biais du comblement de la cavité au moyen de sédiments meubles plus ou moins fins et, pour partie, d'origine anthropique. Le résultat de ce mélange est donc une couche sédimentaire majoritairement composée d'une matrice limoneuse. À celle-ci s'entremêlent des éléments de taille assez grande. Cette fraction granulométrique, qui a une taille supérieure à $1 \mathrm{~mm}$ de diamètre, comporte plusieurs types de matériaux.

Nous remarquons d'abord de gros grains anguleux de calcaire dolomitique parfois très poreux (fig. 38). Il s'agit de débris et fragments du calcaire local mélangés aux sédiments fins. Leur origine est sans doute naturelle, due 


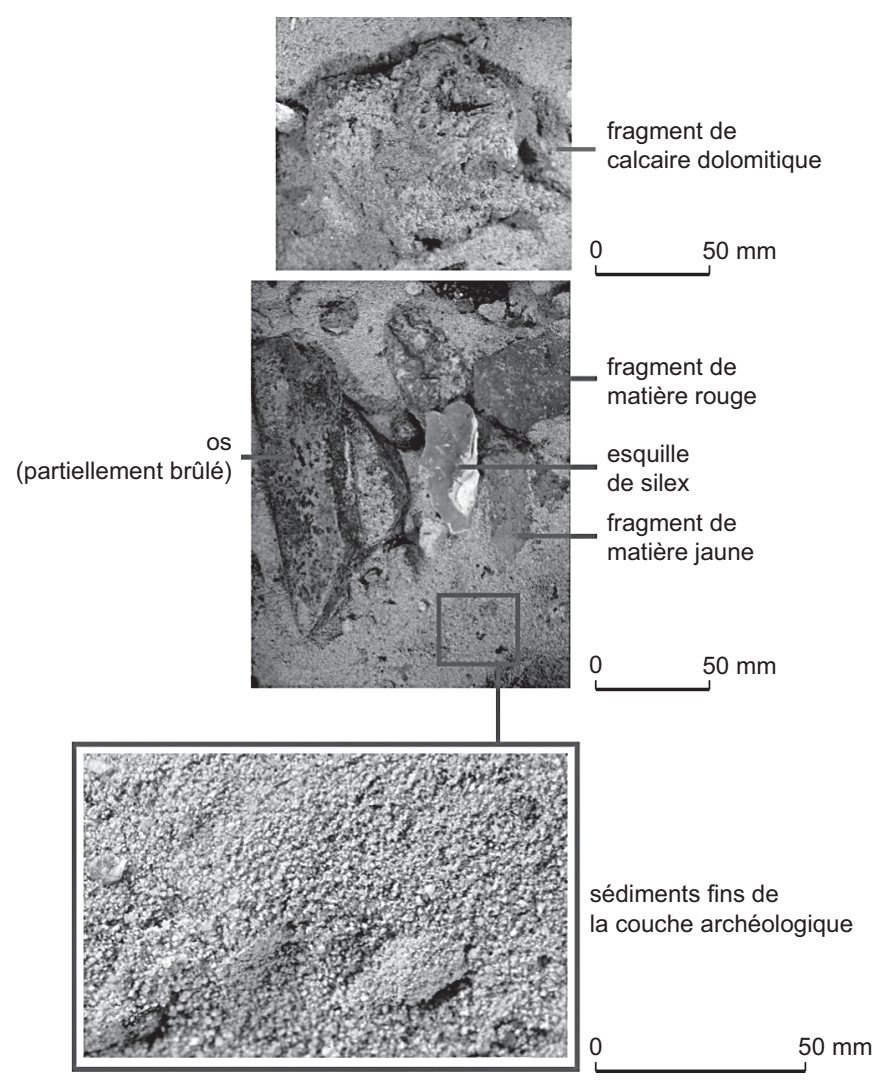

Fig. 38 - Macrophotographies des sédiments de la couche archéologique de la Marche: macrorestes et sédiments fins (clichés: D. Bagault, Centre de recherche et de restauration des musées de France).

à la desquamation et à la fragmentation de la paroi et de la voûte de l'abri.

D'autres éléments, de taille assez grande (de $1 \mathrm{~mm}$ jusqu'à plus de $10 \mathrm{~mm}$ ), apparaissent: ce sont surtout des fragments d'os. Parfois (mais pas toujours), il s'agit d'os brûlés. L'ancienneté de ces os est assurée par l'existence d'une couche stérile qui recouvre la couche archéologique dans laquelle ils ont été trouvés. Nous pouvons de ce fait être certain que ces vestiges ne se sont pas infiltrés depuis les couches plus récentes. Dans le même ordre d'idées, d'autres objets témoignent de l'activité humaine: c'est le cas de quelques petites esquilles de silex trouvées dans l'échantillon de sédiment. Ces macrorestes nous démontrent donc suffisamment qu'il y a eu présence humaine au sein du site et que celle-ci a eu un rôle sur la formation sédimentologique de la couche étudiée.

La fraction granulométrique inférieure à $500 \mu \mathrm{m}$ est quant à elle composée de grains anguleux, la plupart du temps beiges ou jaunâtres parfois rougeâtres ou d'une teinte marron. Sa composition chimique nous montre une présence importante d'argile $\left(\mathrm{Al}_{2} \mathrm{O}_{3} \mathrm{SiO}_{4}\right)$, élément fréquent voire omniprésent dans les sédiments (fig. 39). Nous avons de plus constaté l'importance de la dolomite $\left(\mathrm{CaMg}\left[\mathrm{CO}_{3}\right]_{2}\right)$ dans ces derniers (fig. 40). En effet, tous les échantillons contiennent aussi bien du magnésium $(\mathrm{Mg})$ que du calcium $(\mathrm{Ca})$ et du carbone $(\mathrm{C})$. Ceci est tout à fait normal car les falaises environnantes sont composées de calcaire dolomitique, ce qui explique que les grains de dolomite soient un composant majeur des limons argileux.

En ce qui concerne l'oxyde de fer, on constate que lui aussi est présent dans les sédiments, bien qu'en concentration moindre que l'argile et la dolomite. C'est sans doute cet oxyde qui joue un rôle important dans le rougissement du sédiment. Certains échantillons (36\%) montrent également la présence de manganèse (Ma) qui pourrait être lié à la composition naturelle du remplissage.

Quelques éléments plus rares se manifestent aussi dans le spectre chimique du dépôt. C'est notamment le cas du potassium qui semble être omniprésent, bien qu'en faible quantité, dans les sédiments de la Marche. Nous tenons par ailleurs à attirer l'attention sur l'absence totale de chlore (Cl) et de soufre (S) dans les sédiments de la couche archéologique. Ceci se révélera par la suite intéressant pour la comparaison envisagée avec les matières colorantes prélevées.

Mais avant de pouvoir effectuer ce travail, il convient d'abord de livrer les résultats concernant les pigments prélevés.

\section{Analyse des pigments prélevés sur les pierres}

Comme nous l'avons déjà signalé plus haut (voir supra), des pigments de trois teintes ont été repérés sur les pierres de la Marche: le rouge, le noir et le jaune. Ces teintes sont plus ou moins fortement représentées. Les pigments ont été échantillonnés de manière représentative afin de pouvoir analyser de manière plus approfondie les compositions de ces matières colorantes; grâce à cela, on peut espérer déterminer leur nature et obtenir des indices sur leur origine. Par commodité, les résultats seront énoncés couleur par couleur.

\section{Le rouge}

Les pigments rouges sont chargés en oxyde de fer. C'est ce dernier qui donne d'ailleurs la coloration rouge. Les pouvoirs colorants de cet oxyde sont bien connus: il suffit d'une petite quantité pour colorer une matière. Dans la 


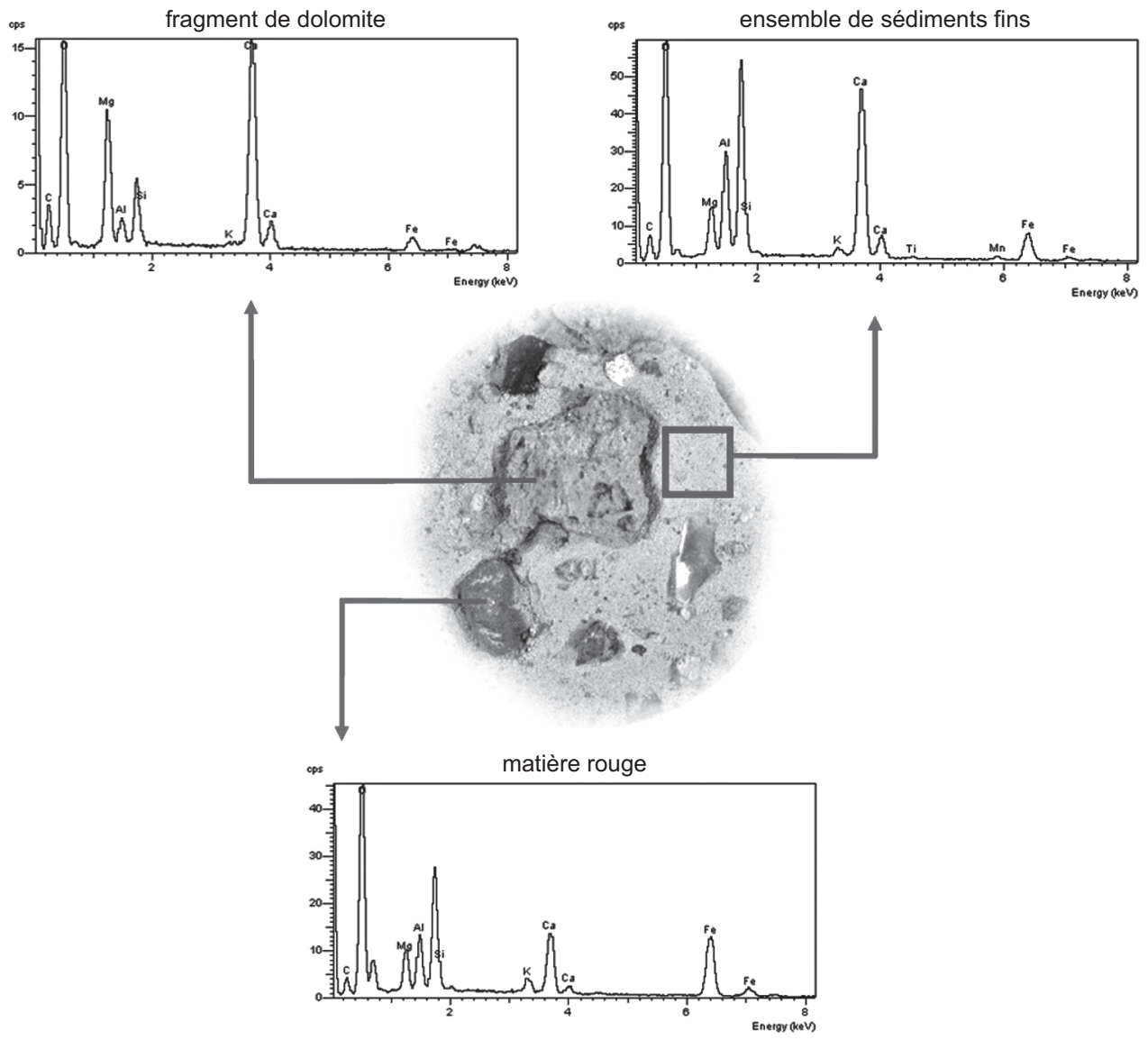

Fig. 39 - Composition chimique des macrorestes dans les sédiments de la couche archéologique de la Marche.

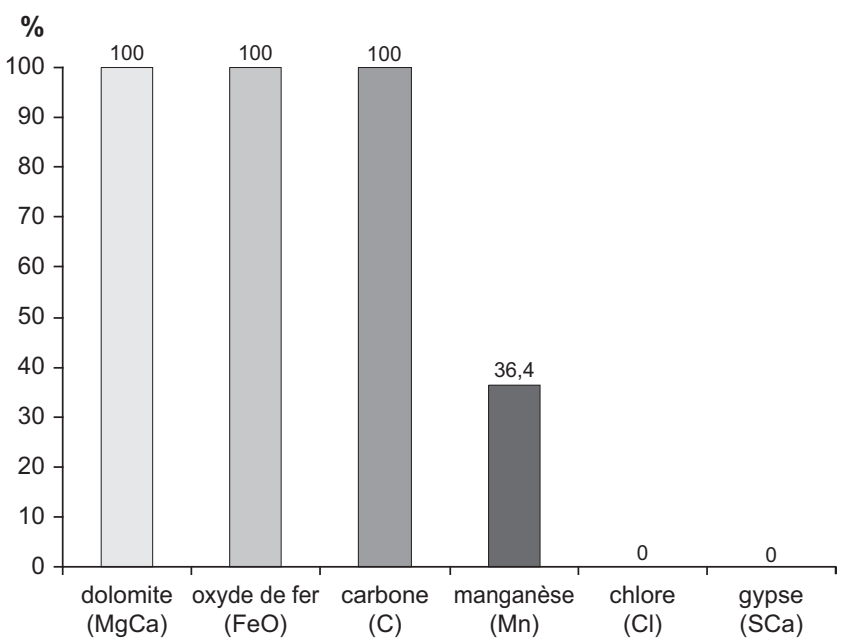

Fig. 40 - Composants chimiques majeurs des sédiments fins de la couche archéologique (sur un total de 11 échantillons analysés). 


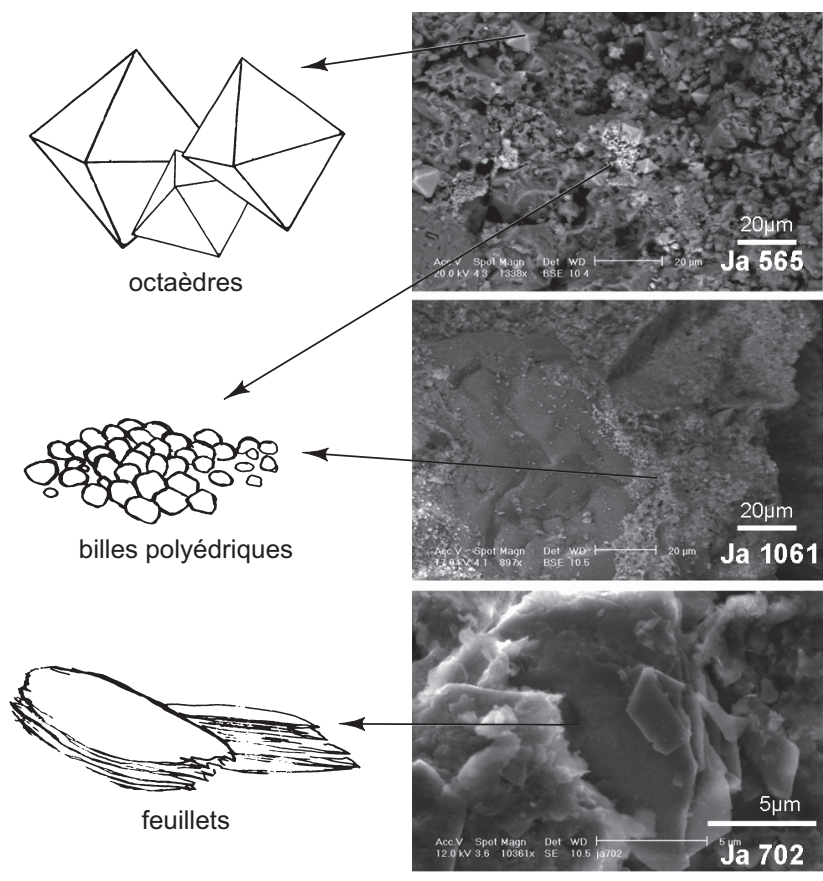

Fig. 41 - Les différentes formes d'oxyde de fer rencontrées dans les échantillons de pigments rouges sur les pierres de la Marche : images MEB (à droite) et schémas (à gauche).

plupart des échantillons, il est ainsi difficile d'identifier l'agencement exact de l'oxyde dans la matrice (argileuse). La forte présence d'argile et la dispersion des oxydes de fer semblent indiquer que nous avons plus affaire à des ocres (terres rouges) qu'à des hématites comme matière première.

Dans certains cas, il nous a cependant été possible de localiser les oxydes de fer et de les caractériser. Nous avons ainsi répertorié trois types de morphologie des cristaux (fig. 41).

Le premier, observé sur plusieurs pierres, est celui de petits cristaux en billes facettées et de dimensions assez réduites $(1 \mu \mathrm{m})$. Ces billes peuvent être agglomérées ou solidaires. Dans ce dernier cas, elles sont difficilement repérables dans la matrice.

Le deuxième type rencontré fut repéré sur une seule et unique pierre dans notre échantillon (Ja 565, pl. 48 à 51). Il a la forme d'un octaèdre et les cristaux sont plus grands que ceux du premier groupe (environ $5 \mu \mathrm{m}$ ). Dans ce cas, les octaèdres sont d'ailleurs associés aux petites billes déjà mentionnées.

La troisième forme, enfin, est constituée de feuillets fins et lités possédant des strates d'une épaisseur inférieure à $500 \mathrm{~nm}(0,5 \mu \mathrm{m})$.
En ce qui concerne la composition chimique elle-même, nous n'avons pu repérer que l'oxyde de fer ( $\mathrm{FeO}$ ) comme matière colorante donnant des teintes rouges (fig. 42 et 43). L'argile $\left(\mathrm{Al}_{2} \mathrm{O}_{3} \mathrm{SiO}_{4}\right)$ est certes omniprésente mais ne figure pas dans les éléments associés significatifs pour les matières colorantes. La plupart des échantillons montrent la présence de l'oxyde de fer soit seul soit en combinaison avec la dolomite. Il est cependant remarquable que 11 cas présentent un oxyde de fer associé au chlore $(\mathrm{Cl})$ et 6 cas au gypse $\left(\mathrm{CaSO}_{4}\right)$. Le chlore n'est certainement pas présent en tant que tel dans les pigments; il s'agit plutôt d'un type de chlorure (forme de sel). Il ne nous a cependant pas été possible de déterminer avec certitude l'autre élément qui indiquerait la nature exacte de celui-ci.

\section{Le "faux noir"}

Lors de l'échantillonnage nous nous sommes aperçus que certains pigments classés comme noirs dans l'inventaire ont en réalité une teinte rougeâtre. Ceci est devenu une évidence lors de l'examen détaillé des échantillons sous la loupe binoculaire au laboratoire. L'analyse des spectres au MEB a montré que ces « faux noirs » sont en fait des rouges très foncés et basés sur la même composition chimique que les rouges. Ceci a par exemple été le cas pour le «noir »de la pierre Ja 565 (fig. 44).

Dans les sept observations effectuées, l'oxyde de fer est associé à un chlorure.

\section{Le noir}

Les échantillons noirs montrent, eux, une composition plus complexe que les pigments rouges. Les matières colorantes de base sont de deux natures différentes: le charbon, avec son composant majeur, le carbone $(\mathrm{C})$, et le manganèse (Mn) (fig. 45). Ce dernier, certainement présent sous forme d'oxyde, est, sur une pièce, associé à un chlorure (attesté par la présence de $\mathrm{Cl}$ ). Le carbone se trouve quant à lui en majorité seul ou lié à de la dolomite $\left(\mathrm{CaMg}\left[\mathrm{CO}_{3}\right]_{2}\right)$. Dans un cas nous le retrouvons associé à un chlorure, dans quatre au gypse.

Les matières colorantes noires sont souvent difficilement localisables sur les images car elles semblent être dispersées en très petits fragments dans la matrice. Dans les cas où le carbone a été localisé en grande concentration sur les images en contraste chimique du MEB, nous pouvons observer des fragments de charbons, même s'ils restent difficilement identifiables. À ce titre, un des spécimens, qui semble correspondre à un charbon d'après sa porosité et sa structure, a été plus particulièrement étudié (fig. 46). Le 

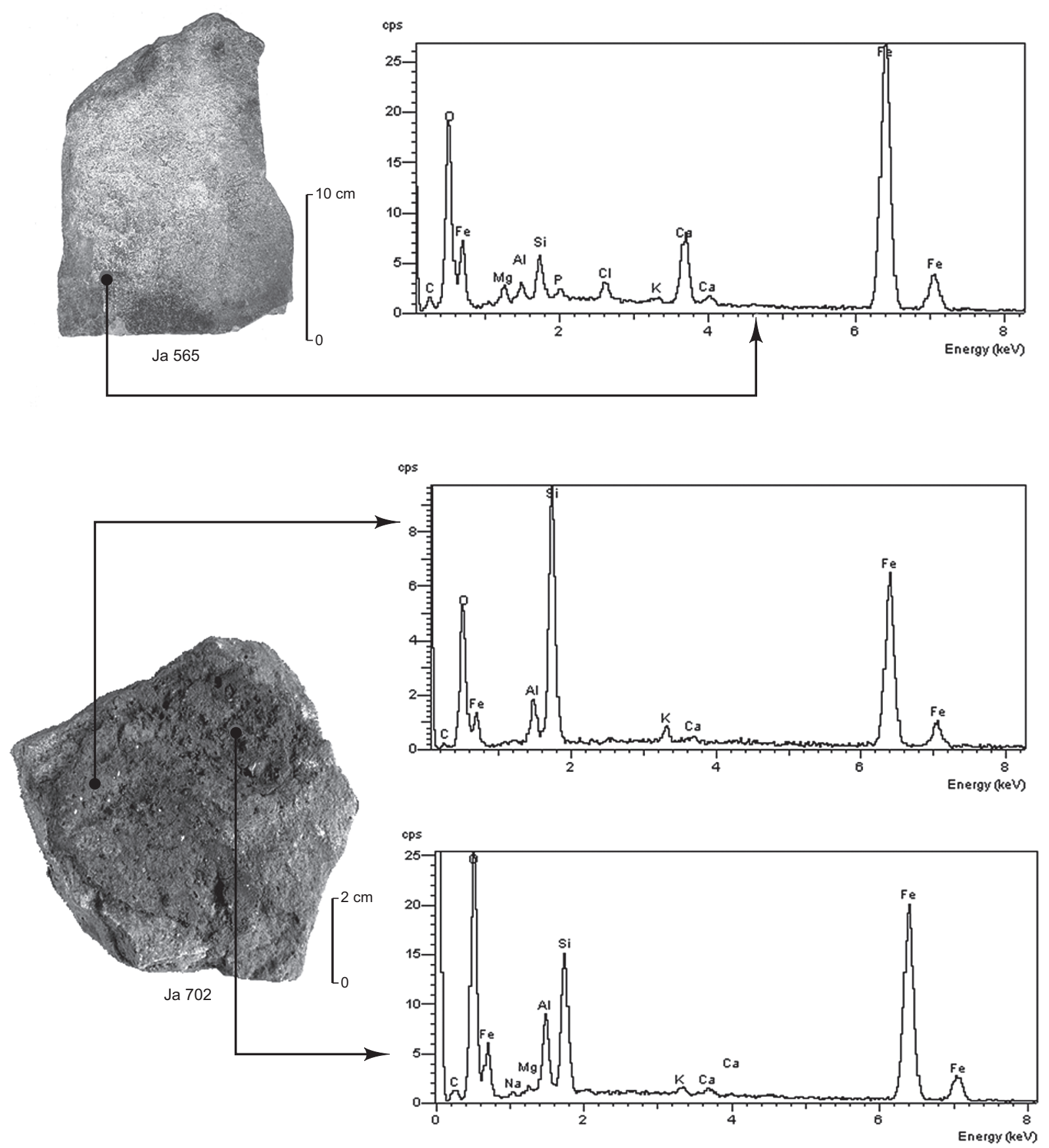

Fig. 42 - Composition chimique des pigments rouges sur deux pierres du site de la Marche : plaque peinte (en haut) et «meule» (en bas).

fragment mesure $20 \mu \mathrm{m}$ de longueur et possède des pores de tailles variables (entre $10 \mu \mathrm{m}$ et $1 \mu \mathrm{m}$ ). Il semble s'agir de pores d'une matière organique qui a été brûlée. Pour déterminer sa nature précise, nous avons, comme comparaison, réalisé une microscopie sur deux échantillons, l'un de charbon de bois et l'autre de charbon d'os (fig. 46). Le charbon d'os possède des pores très larges, supérieures à $100 \mu \mathrm{m}$ et allant jusqu'à $500 \mu \mathrm{m}$. Le charbon de bois, quant à lui, possède des pores de tailles beaucoup plus réduites, inférieures à $20 \mu \mathrm{m}$. S'il s'agit bien dans notre cas d'un fragment de pores, il est donc à notre avis plus probable que ce charbon soit un charbon de bois. 


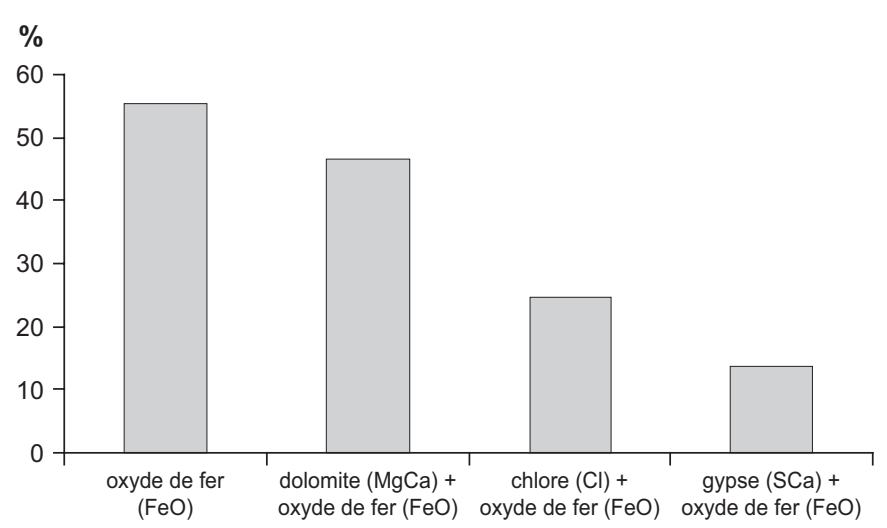

Fig. 43 - Associations majeures d'éléments chimiques parmi les pigments rouges.
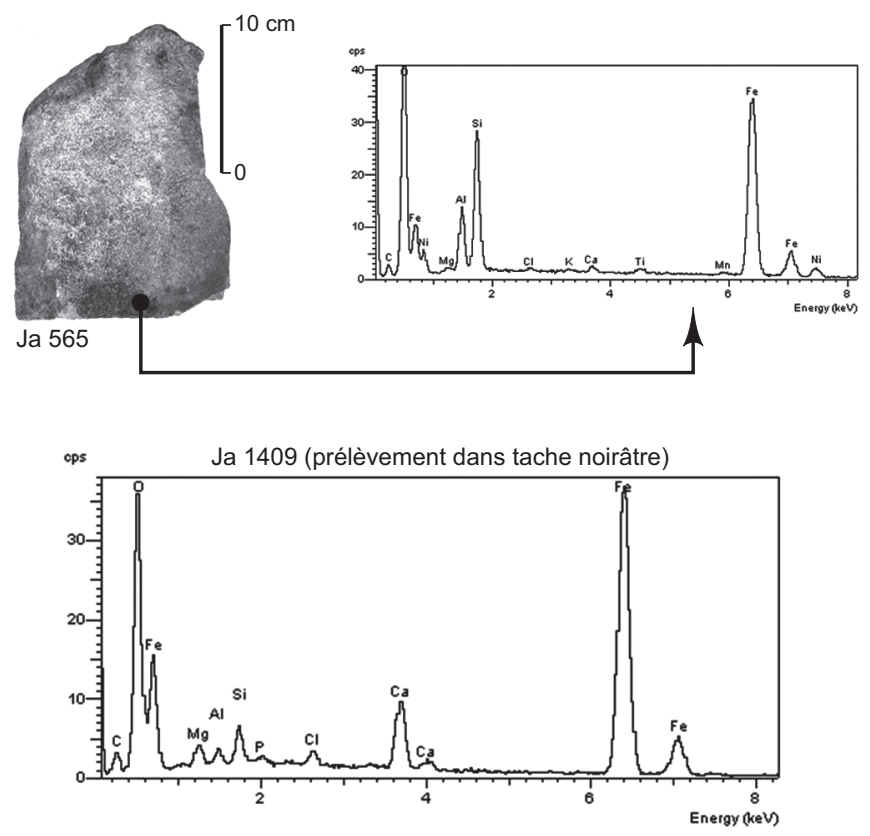

Fig. 44 - Composition chimique d'un "faux noir » associé à des pigments rouges. Celui-ci se révèle être un pigment à base d'oxyde de fer.

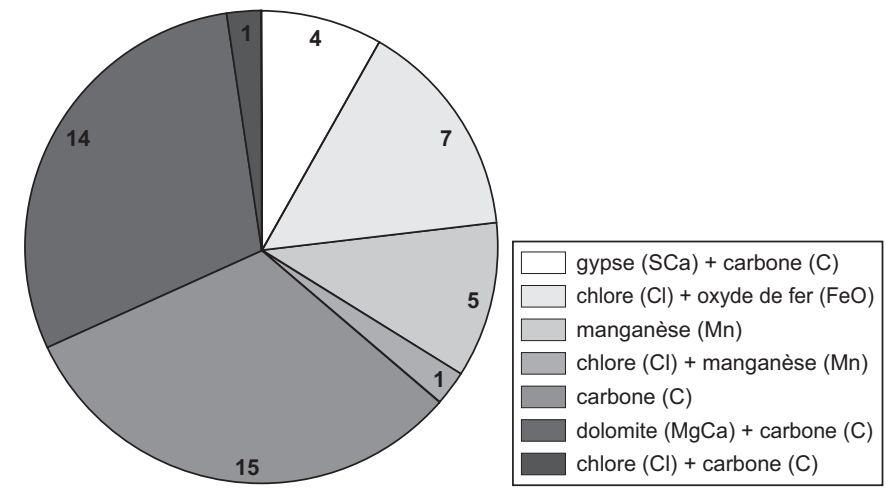

Fig. 45 - Associations majeures d'éléments chimiques parmi les pigments noirs.

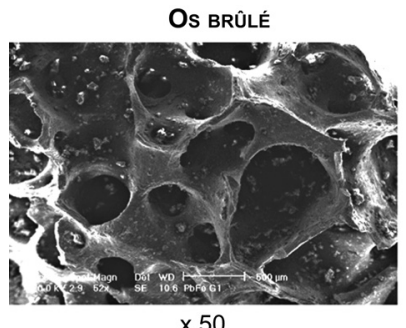

$\times 50$

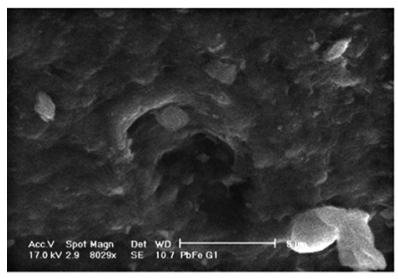

$\times 8000$

Fig. 46 - Comparaisons d'un charbon d'os de la couche archéologique et d'un charbon de bois de chêne de l'époque médiévale. En bas à droite, éléments charbonneux dans un pigment noir sur une des pierres de la Marche.

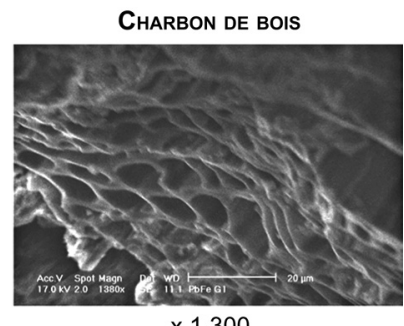

$\times 1300$

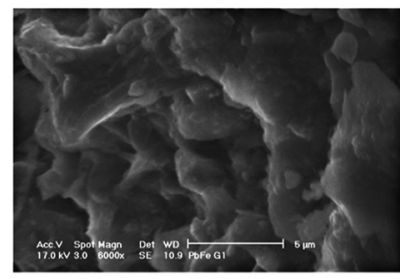

$\times 6000$

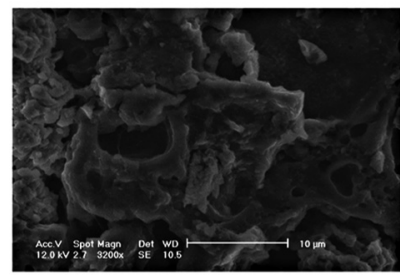

$\times 3000$ 


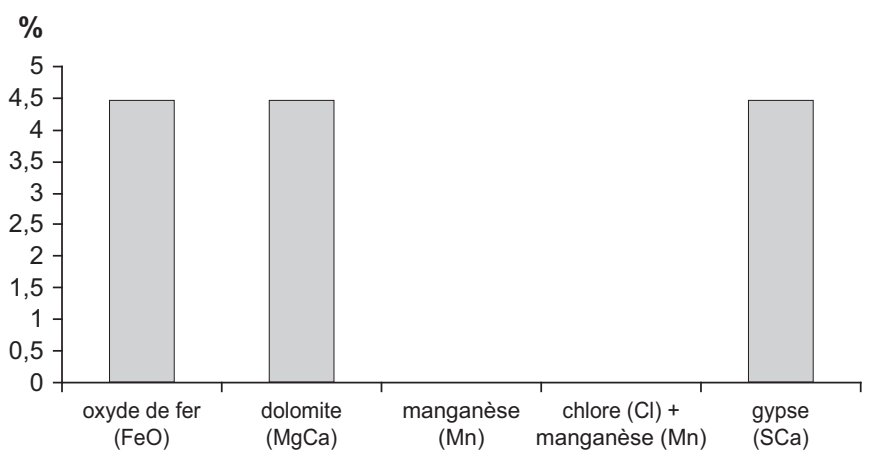

Fig. 47 - Associations majeures d'éléments chimiques parmi les teintes marron.

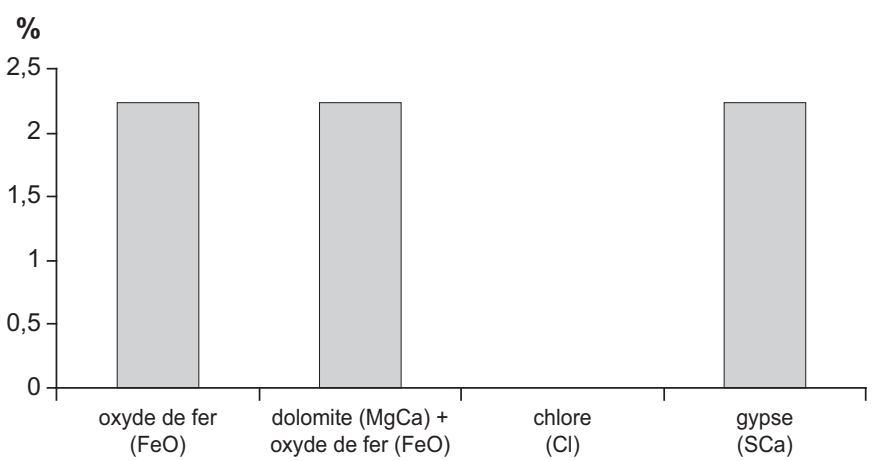

Fig. 48 - Associations majeures des éléments chimiques parmi les pigments jaunes.

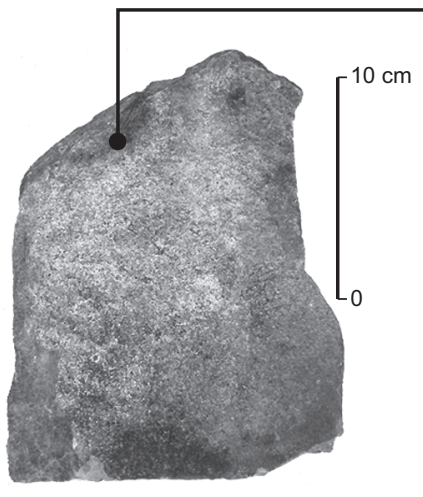

Ja 565

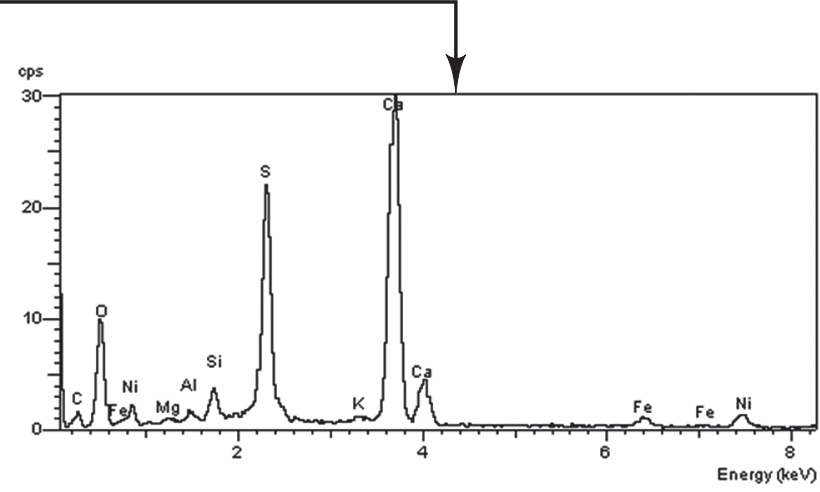

Fig. 49 - Composition chimique d'un pigment jaune sur une des pierres de la Marche.

\section{Le marron}

Le marron a été repéré sur 18 pierres parmi les 1049 que nous avons étudiées. D'un point de vue chimique les teintes marron sont composées de gypse $\left(\mathrm{CaSO}_{4}\right)$ et d'oxyde de fer (fig. 47), comme c'est le cas pour le jaune (voir infra). Le manganèse ainsi que le chlore sont absents.

Sans doute s'agit-il d'une teinte qui est une variation du rouge ou du jaune et nous ne considérons pas de ce fait le marron comme une couleur spécifique.

\section{Le jaune}

Le jaune est assez rare parmi les pigments repérés: seules quatre pièces de la Marche montrent des pigments réellement jaunes. Dans ce lot se trouve un bloc de matière assez tendre qui s'est révélé être un gypse $\left(\mathrm{CaSO}_{4}\right)$.

La composition chimique du jaune lui-même est assez simple: les deux échantillons que nous avons étudiés montrent la présence d'oxyde de fer et de dolomite (MgCa CO). Ils sont probablement constitués de limonite
$(\mathrm{FeO}[\mathrm{OH}])$ sous forme de gothite $\left(\mathrm{FeO}-\mathrm{OH} \cdot \mathrm{nH}_{2} \mathrm{O}\right)$, un oxyde de fer hydraté mélangé à de l'argile (fig. 48 et 49). La dolomite, quant à elle, provient essentiellement des sédiments voisins. D'autres échantillons, contenant du soufre, ne semblent pas être des matières locales, sans que l'on puisse définir avec certitude leur origine. Une fois encore nous notons l'absence de chlore dans tous ces échantillons.

\section{NATURE ET ORIGINE DES PIGMENTS}

Il est certain que l'étude au MEB des pigments ne répond pas à toutes les questions concernant la nature et la composition des constituants. Notre étude a cependant éclairé certains détails qui nous ont permis de mieux nous approcher de la nature et de l'origine des pigments.

Nous avons d'abord noté la présence constante de l'argile comme matériau constituant la matrice dans laquelle sont encastrées les matières colorantes. Cependant, comme 
l'argile est un minéral très fréquent dans les sédiments, il n'est pas possible de parler de « recette » de préparation des pigments. De même, malgré l'homogénéité relative de cette matrice de base, chaque pigment montre des compositions intéressantes à noter.

Le noir est surtout composé de charbon: nous avons pu identifier des charbons de bois dans deux pigments étudiés. En revanche, certains noirs rencontrés sont constitués de manganèse (6 cas). La provenance de ces deux composants est sans doute d'origine anthropique.

L'intentionnalité réelle de l'apport du noir de charbon, du moins dans le but de colorer la surface de la pierre, reste quant à elle incertaine. Il est en effet possible que les pierres aient été placées près des foyers, en contact direct avec les charbons, et que la coloration ait été entraînée par cette proximité. Pour l'oxyde de manganèse, l'apport anthropique est en revanche assuré, car il est certes présent dans les sédiments du site mais ne semble pas se trouver sous forme d'éléments grossiers supérieurs à $5 \mathrm{~mm}$; il n’a donc pas pu se déposer de manière naturelle sur la surface.

Pour ce qui est du marron et du jaune, nous avons constaté qu'il s'agit de mélanges d'argile avec présence de gypse, qui est sans doute un matériau extra-local. Nous avons certes trouvé de petits fragments de cette matière, sous forme de grains assez grossiers (> $1 \mathrm{~mm}$ ), dans les sédiments. Cependant aucune trace de gypse n'a pu être détectée dans les sédiments fins: il n'y a donc pas eu présence naturelle de ce composant dans le site, puisque celle-ci aurait suscité automatiquement une altération et le mélange des composants du gypse en fragments infimes aux sédiments fins. Il semble donc que ce matériau ne fasse pas partie des constituants naturels des sédiments de la couche archéologique. Faute d'exemples valables pour une comparaison, les raisons et le type d'emploi de cette matière par les Magdaléniens de la Marche restent incertains.

Nous avons vu que le rouge est composé d'un mélange d'argile comme les autres pigments, parfois de dolomite et d'oxyde de fer. Les deux premiers composants constituent la majeure partie des sédiments de la couche archéologique. L'oxyde de fer est également présent dans la couche archéologique mais en concentration bien moindre que dans les pigments rouges. Cet indice nous montre qu'il ne s'agit pas d'une coloration due aux sédiments environnants mais plutôt d'un apport d'origine anthropique.

La question de la provenance des matières colorantes, notamment du rouge et du jaune, est difficile à aborder car nous n'avons pas pu trouver de gisement précis, proche du site et susceptible d'être la source des matières premières.
Cependant, pour le rouge, des ocres et d'autres argiles riches en oxyde de fer sont assez fréquentes dans la nature. Il n'est donc pas forcément nécessaire d'avoir eu recours à un seul gisement d'approvisionnement.

Par ailleurs, nous avons trouvé la preuve que certains matériaux semblent être exogènes au site de la Marche. Nous avons en effet évoqué la présence de chlorure indéterminé (voir supra) associé au manganèse (1 cas), au carbone (1 cas), et surtout à l'oxyde de fer (11 cas). Or, les chlorures sont totalement absents des sédiments de la couche archéologique. Il semble donc qu'ils soient, dans le cas du rouge, le marqueur de la provenance du matériau; celle-ci, certainement distincte de celles des autres colorants, doit par ailleurs être extérieure au site de la Marche. En effet, les matériaux des autres pigments peuvent, quant à eux, avoir été prélevés dans les abords immédiats du site.

Nous tenons aussi à attirer l'attention sur une plaque assez particulière en ce qui concerne l'utilisation des pigments (Ja 565, pl. 48 à 51). L'étude des pigments rouges a, dans ce cas, montré que l'oxyde de fer est associé à la présence d'un chlorure. Mais cette plaque montre de plus la présence d'une forme d'oxyde de fer assez particulière, celles de l'octaèdre (fig. 41). D'autres oxydes du pigment se présentent sous forme de billes, ceci étant la forme la plus courante parmi les constituants de l'oxyde de fer. Ce support est donc pour le moment un cas unique parmi les pierres de la Marche. À cette originalité des pigments s'ajoute celle de la matière première même du support. En effet, cette plaque est très homogène en ce qui concerne sa granulométrie et possède aussi deux faces parfaitement planes. Il est fort probable que ce support appartienne à un faciès de calcaire exogène au site, celui que l'on nomme kimméridgien. L'association de ces deux éléments montre donc qu'il s'agit d'une pierre qui a fait l'objet d'une attention et d'un traitement particulier.

Enfin, au terme de cette analyse, nous constatons que l'apport des pigments, notamment des rouges, est essentiellement d'origine anthropique. Ceci confirme l'observation faite sur d'autres supports colorés. Nous pensons notamment ici aux petits blocs de matières courantes portant des stries d'abrasion (pl. 52 et 53). La microtopographie met bien en évidence ces groupements de stries sur le petit bloc noir (pl. 52). Celles-ci mesurent en général entre $10 \mu \mathrm{m}$ et $20 \mu \mathrm{m}$ de profondeur. Parfois, un ensemble de traces a creusé l'intégralité de la surface: les extractions de profils laissent en effet apparaître des vallées de plus d'un millimètre de largeur et $100 \mu \mathrm{m}$ de profondeur. À l'intérieur de ces vallées, nous retrouvons les stries fines. 


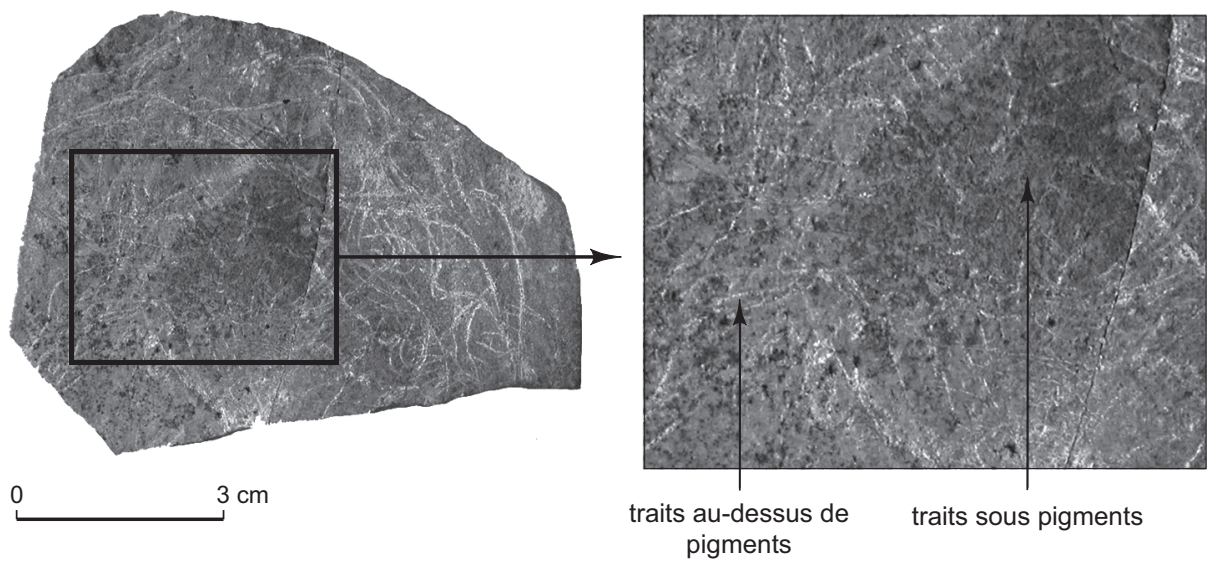

Fig. 50 - Pierre gravée peinte de la Marche, avec interrelations entre gravures et aplats de couleurs (cliché : N. Mélard).

Il semble que certaines d'entre elles se soient produites en même temps que les traces plus marquées; d'autres au contraire recoupent ces dernières. Nous observons enfin des zones plus accidentées sous forme de creux de $100 \mu \mathrm{m}$ de profondeur environ.

Ces observations témoignent de l'utilisation variée de ces supports. Ainsi les traces profondes proviennent-elles sans doute d'un frottement des pièces sur un matériau grossier tandis que les stries fines révèlent plutôt une interaction avec une matière abrasive elle-même plus fine. Les creux possédant une surface plus grossière, quant à eux, pourraient provenir de chocs par percussion.

Qui plus est, la forme globale de ces pierres, qui évoque une goutte arrondie, est clairement le résultat de cette abrasion qui, d'après les études des microsurfaces, semble provenir d'un frottement régulier; ce dernier a en effet affecté toutes les surfaces de l'objet.

Une chose intéressante à noter est que ces deux objets, qui sont identiques au niveau de leur forme, sont constitués de deux matières différentes. Il s'agit, pour l'un, d'un petit bloc riche en oxyde de fer (hématite?) et d'un bloc riche en manganèse, pour l'autre. Leur utilisation paraît avoir été la même: ils ont pu être employés pour la réalisation d'aplats de couleur ou la production de poudre colorée. Cette dernière opération est attestée par un autre objet, bien que le procédé soit différent. Une pierre a en effet probablement servi de meule pour piler des matières colorantes (fig. 42).

$\mathrm{Si}$ cette origine anthropique des colorants fait donc moins de doute, il faut aussi noter qu'il est improbable qu'elle soit moderne: les analyses complémentaires effectuées au MEB montrent en effet qu'en général les pigments adhèrent bien à leur support et que certains pigments sont anciens puisqu'ils sont recouverts d'une couche de calcite. À partir de cette observation, il semble donc valable d'effectuer une analyse du ou des rapports qui existent entre les gravures et les colorants.

Plusieurs plaquettes portent à la fois des gravures et des traces de pigments. Certaines nous permettent d'étudier de manière plus approfondie leurs rapports.

Une pièce est, à ce titre, particulièrement intéressante. Il s'agit d'un fragment de plaquette d'environ $10 \mathrm{~cm}$ de diamètre. Toute sa surface est couverte de rouge mais également de traits fins gravés (fig. 50). Une observation plus détaillée fait apparaître des endroits où le pigment remplit les vallées des incisions. Dans d'autres, nous observons au contraire que les tracés ont été gravés sur le pigment. La lecture et la compréhension intégrales du palimpseste sont très compliquées et nous n'avons pas pu déchiffrer entièrement ce support. On retiendra l'intérêt cependant de cette chronologie relative.

Une autre pierre montre que les traits gravés sont intégralement couverts d'une patine rouge. Cette dernière n'a cependant pas pu être étudiée d'un point de vue chimique car le prélèvement de matière s'est révélé trop risqué pour la conservation de la pierre.

Le rapport entre gravure et pigment existe donc réellement et paraît particulièrement intéressant lorsqu'on s'interroge sur la superposition des motifs telle qu'on la constate dans de nombreux cas aussi bien à la Marche que dans d'autres sites comme Gönnersdorf et Enlène (Bosinski, Fischer, 1974; Clottes, 1999; Bosinski, 2006). Ces analyses, ainsi que toutes les autres déjà mentionnées au cours de cette étude, nous permettent donc maintenant d'envisager des pistes de réflexion pour l'interprétation des pierres de la Marche. 


\section{INTERPRÉTATIONS}

Comme nous l'avons déjà souligné à plusieurs reprises, les gravures constituent le cœur de notre étude. Voilà pourquoi nous nous efforcerons, dans le cadre de cette tentative d'interprétation, de débuter par elles. À ce titre, trois points seront successivement abordés, qui sont d'abord la maîtrise du graveur dont elles témoignent, mais aussi le soin d'exécution qu'elles reflètent et enfin le sens que l'on peut dégager de leurs motifs.

\section{L'ART DE LA GRAVURE À LA MARCHE}

Un art maîtrisé, c'est-à-dire des supports variés mais choisis par des Magdaléniens familiers avec les matières lithiques.

La maîtrise des graveurs magdaléniens peut d'abord s'évaluer à partir de l'étude des supports. En effet, ceux-ci témoignent souvent d'un choix précis par le graveur en fonction de leur taille et/ou de leurs propriétés mécaniques. L'homme préhistorique possédait donc une connaissance précise du matériel à utiliser pour la gravure.

Nous pouvons remarquer qu'il existe une grande variété de dimensions des supports; deux types se distinguent nettement: les supports manipulables de petites et moyennes dimensions, d'une part, et les grandes dalles et blocs lourds, d'autre part. Les graveurs magdaléniens de la Marche ont donc réussi à utiliser avec la même dextérité des supports qui, par leurs différences de poids et de mobilité, ne réclamaient certainement pas le même traitement.

Les grandes dalles (supérieures à $30 \mathrm{~cm}$ de longueur et 5 mm d'épaisseur) qui ont été utilisées comme supports de gravure montrent une pétrographie identique aux roches affleurant à l'intérieur de l'abri ainsi qu'à proximité du site, dans la vallée. Ces dalles et gros blocs sont sans doute des fragments de la paroi de l'abri qui se sont détachés de la falaise. Leur état de surface brut montre en effet un aspect altéré typique des parois environnantes et des surfaces dégagées des couches de calcaire.

Même s'il est possible que certains blocs aient été gravés directement, et peut-être même accrochés à la paroi, certaines dalles au contraire ont sans doute été déplacées car celles-ci sont gravées des deux côtés. L'hypothèse selon laquelle les gravures auraient été gravées à même la paroi est difficile à certifier car aucun vestige de gravure sur celle-ci ne témoigne de leur présence ancienne. Il semble donc que tous les supports étaient déjà détachés de la falaise.
Sur ces dalles et blocs, nous observons souvent des figures assez grandes qui, parfois, s'inscrivent bien dans la forme de leur support. Les grandes dalles notamment, qui ont été étudiées par L. Pales, sont généralement gravées de motifs de taille importante (Pales, Tassin de Saint-Péreuse, 1969, 1976, 1981 et 1989). À ceux-ci s'ajoutent souvent de denses entrecroisements de traits. Ces gravures sont réalisées sur les faces planes des supports qui sont en fait des surfaces altérées ou bien des plans de clivage assez peu accidentés. C'est sans doute pour cela qu'ils ont été choisis comme supports de gravure. Quant à leur dénomination, nous pensons que l'expression «parois mobiles ", introduite par l'abbé Breuil, est assez juste pour désigner ces pierres.

Les prospections sur le terrain ont également montré que le type des petites plaquettes planes et homogènes en surface et en épaisseur est très rare à proximité du site. En effet, le calcaire bathonien affleure sous forme d'épaisses couches de calcaire dur et assez irrégulier, enfermant parfois des poches de sable. Dans certains endroits du versant, il est cependant possible de repérer quelques filons composés de plaquettes plates, propices à la gravure: pour trouver celles-ci, il était sans doute nécessaire que les Magdaléniens parcourent un certain temps le site. Les pièces ont éventuellement pu être ramassées lors de déplacements courts autour du site en vue de les utiliser ultérieurement comme support de gravures. Cela montre bien que celles-ci n'ont pas été réalisées sur n'importe quel support à disposition, mais que le choix de ce dernier a donné lieu à une recherche assez poussée. Les caractéristiques de la matière entraient donc en ligne de compte pour le graveur. De fait, la plupart des gravures, notamment celles qui sont de très bonne facture, se trouvent sur des surfaces planes. C'est sans doute la planéité qui constituait une des caractéristiques décisives pour le choix des supports.

En ce qui concerne les motifs présents sur ces supports de petites et moyennes tailles, il est remarquable qu'ils soient tout à fait semblables à ceux des grands. De plus, il n'existe pas toujours de lien direct entre la taille du support et celui du motif. Dans certains cas, nous trouvons de petites figures sur une surface assez grande; dans d'autres cependant, les motifs s'inscrivent bien dans les limites des supports et leur taille est adaptée. Nous avons parfois même affaire à un véritable " cadrage ". Un exemple très net est celui d'une représentation de renne (pl. 6 à 9). Le contour de l'animal occupe toute la surface disponible. On ne peut pourtant pas déterminer dans ce cas si c'est la forme de la plaquette qui a motivé l'inspiration ou bien si un support adapté a été recherché pour le motif. Par ailleurs, on peut 
signaler que les représentations figuratives ne sont pas les seules concernées par un dialogue graphique étroit avec le support. Les figures abstraites peuvent l'être également.

Certaines gravures nous montrent aussi que l'artiste a quelquefois été inspiré par des formes naturelles préexistantes sur les supports. Ainsi une vulve a-t-elle été gravée de manière à ce que certains éléments du motif soient issus de la forme naturelle de la surface (pl. 32). Cette utilisation graphique du relief naturel est beaucoup plus fréquente dans l'art pariétal que dans l'art mobilier, où il apparaît plus épisodiquement: en effet, nous trouvons généralement les motifs sur des surfaces planes où le contrôle du tracé est plus aisé. Sur les faces accidentées, la gravure se limite globalement aux endroits les plus homogènes. Seuls quelques rares échantillons montrent des traits gravés sur une surface fortement accidentée. Dans ces cas, en revanche, les motifs ne sont pas figuratifs et, souvent, il s'agit seulement de quelques rares traits gravés. Cette planéité déjà évoquée a joué un tel rôle dans le choix du support et son traitement que les Magdaléniens de la Marche ont parfois utilisé un faciès de calcaire étranger à celui du site. Celui-ci se distingue nettement d'un point de vue morpho-pétrographique par sa texture, sa finesse et son homogénéité granulométrique.

Ainsi, pour résumer, on peut dire que les graveurs magdaléniens connaissaient parfaitement les propriétés morphologiques et mécaniques des pierres sur lesquelles ils gravaient. L'utilisation de plaquettes plates tout comme le cadrage de certaines figurations sur les pierres montrent que, techniquement, ils maîtrisaient l'acte de gravure et en connaissaient toutes les contraintes. De ce fait, lorsqu'on se trouve en face de gravures sous-dimensionnées, et présentes parfois sur des surfaces rugueuses, peu propices à la gravure, on peut émettre l'idée qu'il s'agit d'un choix de la part du graveur. Les quelques autres types de supports sur lesquels on trouve des gravures renforcent cette interprétation.

À titre d'exemple, on peut citer le cas de quelques galets calcaires. Ceux-ci ont été gravés finement de motifs figuratifs et géométriques (Ja 709, pl. 54). Ils proviennent certainement du lit fluviatile en fond de vallée et ont été apportés dans l'abri.

Mais cette maîtrise des supports ne peut s'expliquer sans faire intervenir plus précisément les techniques du graveur magdalénien elles-mêmes.

Un art maîtrisé, c'est-à-dire des techniques de gravure multiples et adaptées aux supports.

La typologie que nous avons élaborée dans le cadre de ce travail a montré que le graveur de la Marche disposait d'une véritable panoplie de tracés. Ceux-ci témoignent souvent d'un choix mais l'interaction entre le support et l'acte de gravure n'est, bien entendu, pas absente. En effet, chaque support naturel, aussi homogène et lisse soit-il, possède des irrégularités et aspérités de surfaces qui se révèlent plus ou moins problématiques pour la réalisation des gravures.

Les analyses, notamment celles de la microtopographie, ont montré que l'homme a su tenir compte de ces contraintes, car les gravures possèdent une morphologie qui s'adapte en partie aux microformes de la surface. Le graveur a ainsi su contrôler son geste, en effectuant une combinaison de mouvements et de force qui lui assure la maîtrise de son outil sur cette surface dure et irrégulière. Ceci témoigne d'une excellente connaissance des matériaux, aussi bien de ceux qui constituent les supports gravés que ceux dont sont composés les outils de gravure. Face à certains supports, il semble clair que les graveurs ont dû choisir des outils à la fois fins et résistants avant de commencer la réalisation de leurs figurations.

Cette démarche technique réfléchie et élaborée montre que la gravure était pratiquée par des personnes qui avaient une certaine expérience, du moins en ce qui concerne les figurations.

L'artiste magdalénien ne disposait pas seulement de multiples tracés de gravure pour réaliser son œuvre. Notre étude a mis en évidence l'emploi de pigments, puisque l'on note à la Marche la présence de matières premières comportant des traces d'usure ainsi que des supports ayant fonctionné dans le broyage des pigments. Certains éléments nous ont montré que le pigment a été, au moins en partie, utilisé dans le domaine des représentations, notamment pour mettre en valeur les gravures. L'analyse de leur composition montre que les Magdaléniens maîtrisaient également différentes techniques d'élaboration des colorants, ce qui leur assurait une gamme de couleurs assez diversifiée.

Les analyses spectrométriques au MEB ont ainsi montré que ces pigments sont essentiellement des argiles riches en oxyde de fer. Ce mélange est tout à fait typique de l'ocre qui peut prendre une teinte plus ou moins prononcée et/ou variée. Ainsi s'expliquent les «faux noirs » que nous avons détectés sur certaines pierres. D’autres noirs sont cependant constitués de charbon ou de manganèse. Si l'origine du premier peut être expliquée par la position éventuelle des pierres dans un foyer, le manganèse a, quant à lui, sans doute été employé de manière volontaire; nous n’avons cependant pas pu trouver d'éléments qui expliquent pour 


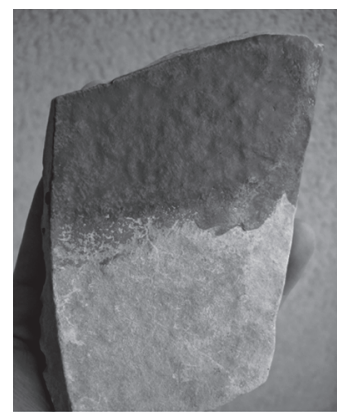

Surface colorée en rouge

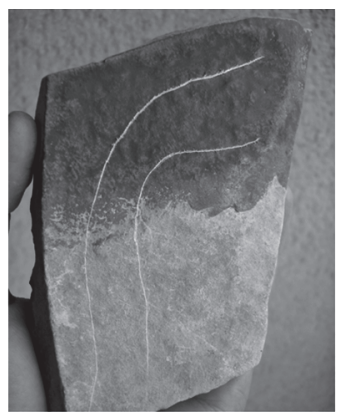

gravure

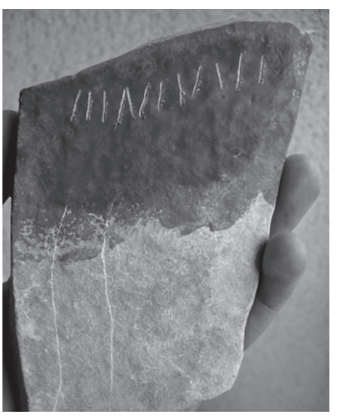

nouvelle phase de coloration et gravure
Fig. 51 - Démonstration de l'utilisation de pigments (ocre et eau) dans la gravure. Les traits gravés sont rehaussés (au centre) ou effacés par l'aplat de couleur rouge (essais et clichés : N. Mélard). quelle raison exacte et de quelle manière ces pigments ont été mis en œuvre.

Des jaunes, qui contiennent de l'oxyde de soufre (S) ont aussi été détectés sur certaines pierres.

Certaines compositions chimiques relevées dans le cadre des échantillons de matières colorantes montrent que ces dernières sont parfois exogènes au site: leur apport est donc artificiel, c'est-à-dire anthropique. Un des marqueurs les plus significatifs de ce fait est la présence d'un chlorure indéterminé, attesté par celle de l'élément chlore, qui n'existe pas à l'état naturel dans les sédiments de la couche archéologique mais qui apparaît dans certains pigments.

Ainsi, bien que certains mélanges de colorants puissent être naturels, l'origine anthropique de certains autres est assurée. Cette observation est d'ailleurs confirmée par la présence de petits morceaux de matières colorantes portant des traces d'usure, qui nous montre que les pigments ont été employés au moyen d'un mouvement de frottement. De plus, ils sont, sur quelques plaquettes, employés de façon à entretenir une relation avec la gravure. La technique d'utilisation est intéressante à mentionner.

Il semble que la plupart des pigments aient été employés sous forme de poudre, peut-être mélangée à de l'eau ou à de l'argile.

Nous n'avons trouvé aucun indice de la technique du dessin au «crayon » (c'est-à-dire qui use directement de petits blocs de pigments) sur les pierres. Tous les colorants retrouvés sur les surfaces se présentent sous forme d'aplats plus ou moins étendus ou sous forme de simples résidus ponctuels. Par conséquent, nous ne pouvons pas nous attendre à la présence de dessins figuratifs en couleurs sur les plaquettes de la Marche et il est, de ce fait, difficile d'interpréter les taches de couleurs sur ces pierres.

Il est cependant possible que les surfaces colorées aient été altérées de sorte que, aujourd'hui, des motifs ancienne- ment présents sur les pierres aient disparu. Ce propos est pourtant complètement hypothétique car nous n'avons pas d'indice indiquant la présence d'un motif quelconque. Le seul élément que l'on puisse avancer est qu'il existe, dans l'art mobilier magdalénien, des cas semblables, comme par exemple sur le site de Riparo Dalmeri, en Italie (Dalmeri et al., 2003a et 2003b).

Qu'il soit peu aisé ou non d'appréhender le sens des aplats de pigments sur les pierres, il n'en reste pas moins que sur quelques-unes, rares, existent des relations nettes entre pigment et gravure. On observe alors un emploi spécifique du colorant: soit il est utilisé pour recouvrir des traits gravés, qui en conséquence vont disparaître dans le fond coloré, soit ceux-ci ont été gravés sur une couche de colorant; ils sont, dans ce dernier cas, très bien visibles sur la surface (blanc sur rouge). Ces deux types de relations entre gravure et pigment créent donc des effets visuels bien distincts pour l'observateur: le colorant est ici employé pour effacer des gravures existantes et ainsi préparer la surface pour une nouvelle gravure, qui en sortira rehaussée.

Il est certain qu'une fois le pigment disparu partiellement ou intégralement par l'altération de la surface, il ne reste qu'un palimpseste de traits avec des figures superposées. Or, il est beaucoup plus facile de lire des gravures sur un fond unicolore: nous avons en effet testé cette technique de gravure sur aplat d'ocres rouges naturelles et il s'avère qu'elles sont beaucoup plus lisibles sur un fond coloré que sur le calcaire brut (fig. 51). Il paraît donc possible que, pour un certain nombre de représentations de la Marche, cette technique ait été employée sans qu'il n'en subsiste de traces aujourd'hui. Toutefois cette hypothèse ne peut vraisemblablement pas être proposée pour l'explication de tous les palimpsestes de la Marche.

De même, cet emploi particulier des pigments n'exclut pas leur utilisation pour d'autres opérations qui ne relèvent pas du domaine artistique. 
Les travaux de J. Airvaux ont en effet livré un grand nombre de pigments. Certains blocs semblent de ce fait avoir été employés comme matières premières, desquelles les Magdaléniens ont extrait les pigments par frottement systématique. D'autres blocs ne portent pas de traces directes d'utilisation. Peut-être certains d'entre eux ont-ils aussi été complètement broyés: la présence d'une pierre interprétée comme meule l'atteste. Celle-ci montre que des morceaux d'ocre et d'hématite ont pu y être écrasés (pl. 45 à 47).

Or, si l'on compare la quantité de matières colorantes disponible sur le site et les cas, assez rares, où l'on trouve un rapport direct entre pigment et représentations gravées, il semble clair que l'ocre a également été employée à des fins plus banales (polissage, travail des peaux...). La question des pigments nécessite de ce fait un approfondissement ultérieur pour pouvoir véritablement cerner leur rôle et leur emploi.

Toujours est-il que le graveur magdalénien a montré, au travers de son ouvre, une véritable connaissance des contraintes de ce domaine, un véritable talent d'adaptation et une indéniable originalité en sachant employer différentes techniques. Il est d'ailleurs notable que ces dispositions aient été mises au service d'un art soigné.

\section{UN ART SOIGNÉ}

L'attention que le graveur a portée à la réalisation des motifs se traduit de différentes manières. Ainsi, nous pouvons d'abord mentionner le choix d'un support adapté à la gravure puis, bien sûr, la qualité globale des motifs.

\section{Provenance du calcaire adapté à la gravure}

Le graveur magdalénien a, comme on l'a déjà signalé, procédé au choix des supports. Or, ce choix l'a apparemment poussé à transporter des pierres adaptées à la gravure jusqu'au site de la Marche, même si celles-ci provenaient initialement d'un lieu assez éloigné.

Un faciès de calcaire dans l'ensemble des plaquettes de la Marche se distingue en effet nettement d'un point de vue morphopétrographique par sa texture, sa finesse et son homogénéité granulométrique. Il est très probable que nous ayons ici affaire à un calcaire kimméridgien provenant de la région de Châtellerault. Aucun autre affleurement n'a pour le moment pu être localisé dans la région. Celui, mentionné par L. Pales, qui aurait comporté des plaquettes homogènes et aurait été proche du site de la Marche, n’a pas pu être retrouvé malgré nos investigations de terrain (Pales, Tassin de Saint-Péreuse, 1976). Il reste possible qu'autour de la Marche existent des affleurements de plaquettes assez bien litées en calcaire bathonien, et il semble même que certaines plaquettes proches du site aient été prélevées par les Magdaléniens. Il est cependant impossible de trouver des plaquettes équivalentes à un calcaire kimméridgien dans la falaise de roches bathoniennes du vallon où se trouve la Marche.

Les Magdaléniens ont dû, par conséquent, apporter des plaques de ce territoire de quelques kilomètres carrés seulement et situé à environ $30 \mathrm{~km}$ de notre site d'étude. Même si les supports ne sont pas très volumineux et lourds, cette distance est assez significative pour être mentionnée. Elle témoigne en effet d'un effort qui montre que l'activité artistique de gravure avait une importance certaine pour les Préhistoriques. Nous remarquons d'ailleurs qu'une plaque assez importante (195 $\mathrm{mm}$ x $148 \mathrm{~mm}$ x $25 \mathrm{~mm}) \mathrm{de}$ ce faciès a pu être trouvée à la Marche. Celle-ci n'a pas été gravée mais elle comporte aujourd'hui encore des traces de pigments (pl. 48 à 51). Il s'agit de colorants d'origine anthropique. Cependant, la signification d'un tel déplacement de cette pierre et celle d'un tel apport de couleurs nous échappent pour le moment.

Pour ce qui est des caractéristiques du traitement global des pierres en calcaire kimméridgien, deux scénarios peuvent tout de même être proposés:

- soit les Magdaléniens ont passé un certain temps dans un lieu où le calcaire kimméridgien affleurait, peut-être dans la région de l'actuelle ville de Châtellerault, pour accomplir certaines tâches: chasse, cueillette, passage... Ils ont, à cet endroit, gravé des pierres qu'ils ont ensuite apportées au site de la Marche;

- soit les pierres ont été apportées au site pour leurs propriétés mécaniques propices à la gravure et elles y ont ensuite été gravées.

Pour le moment, les données ne nous permettent pas de privilégier l'une ou l'autre hypothèse. Mais, quoi qu'il en soit, ces deux propositions reflètent un fort intérêt de la part des Magdaléniens pour la gravure. Celui-ci se retrouve dans le soin apporté à l'acte de gravure lui-même.

\section{Gravures naturalistes parfois très élaborées et dynamiques}

Nous avons évoqué à plusieurs reprises à quel point les gravures de la Marche peuvent être fines: la plupart des motifs figuratifs sont gravés de manière très délicate. 
En effet, le degré d'élaboration de certaines figures animales et humaines, même celles de petite taille, est étonnant. Les graveurs de la Marche ont représenté de petites figures avec un tel souci du détail que leur mise en évidence et l'observation des traces produites nécessitent la mise en œuvre de moyens d'investigation très précis comme la microtopographie: certaines de leurs parties sont en effet à peine visible à l'œil nu. Ces analyses adaptées ont également montré que les hommes préhistoriques ont parfois peaufiné leurs motifs par de légères reprises ou par des tracés préparatoires. Ces derniers sont difficiles à mettre en évidence car, en général, le tracé final efface toute trace du trait préparatoire.

Mais la reprise des gravures aussi bien que les dessins préparatoires ou les détails apportés aux figurations montrent l'importance que l'homme a accordée aux motifs eux-mêmes.

Ainsi, l'habitude qu'avait l'homme préhistorique d'observer son environnement se manifeste de manière nette dans la représentation de nombreux détails anatomiques pour les humains comme pour les animaux.

Les représentations humaines de la région aux alentours du site de la Marche, comme celles d'Angles-sur-l'Anglin, prennent à ce titre une place particulière dans le domaine de l'iconographie humaine du Paléolithique supérieur. Les travaux de L. Pales avaient déjà montré les nombreux détails anatomiques qui ont été représentés sur les pierres de la Marche. Les travaux plus récents de J. Airvaux et nos propres recherches ont confirmé cette observation. À côté des détails anatomiques, les graveurs ont dans certains cas reproduit des éléments de coiffures, de parures et de vêtements.

Pour les animaux, la représentation presque fidèle à la nature est une constante qui s'observe partout dans l'iconographie du Magdalénien. Ici, l'homme a, la plupart du temps, également pris un grand soin à représenter de nombreux détails comme les yeux, le museau, les oreilles, le pelage et les pattes. Parfois on distingue même le changement de pilosité en fonction de la zone du corps de l'animal (pl. 5 à 7). On pourrait prendre pour exemple la représentation de félin (pl. 16 à 19) qui montre bien les degrés d'élaboration des motifs. Le graveur a su donner un aspect très organique à cette représentation en travaillant sur les détails de la tête, notamment sur les oreilles et les yeux. Pareille chose a été observée par L. Pales sur la grande dalle « aux deux mammouths affrontés » (Pales, Tassin de
Saint-Péreuse, 1989). Les deux animaux ont été élaborés de manière très détaillée (fig. 9).

La même rigueur et la même fidélité de la part du graveur magdalénien s'observent sur le reste du bestiaire de la Marche. En effet, les motifs de rennes, de chevaux et de bovidés révèlent un grand souci de vérité de la part de celui qui les a réalisés. Même les très petites représentations sont de ce point de vue étonnantes. La pierre portant la figuration d'un renne montre, malgré sa taille d'à peine $10 \mathrm{~cm}$, une accumulation éloquente de détails, notamment en ce qui concerne son anatomie (pl. 22 et 23). Le soin apporté à cette figure est tel que le graveur n'a pas hésité à varier sa technique de traçage: les bois sont ainsi gravés au moyen d'un tracé simple, qui leur donne un aspect assez "minéral ", tandis que le corps profite lui de tracés multiples qui manifestent le côté plus « organique » de cette partie de l'animal.

Ce rendu «vivant » des représentations figuratives est d'ailleurs également souligné par le dynamisme qu'elles dégagent.

On rencontre l'emploi d'une grande variété de moyens artistiques pour la reproduction du dynamisme des figures. Ceci est particulièrement remarquable chez les figurations réalistes et naturalistes. Les figures schématisées sont, quant à elles, plus statiques.

Il est à ce titre toujours étonnant d'observer les multiples représentations de comportements d'animaux sur ce site, même si on retrouve de manière récurrente ce type de figurations animées au Magdalénien moyen. La plupart du temps cependant, le dynamisme se concentre sur le mouvement de troupeaux ou d'animaux individuels. Or, à la Marche, on trouve aussi des représentations d'animaux manifestant des comportements tout à fait particuliers. Dans certains cas, il est même possible d'interpréter ce comportement par une étude éthologique. Sans rappeler l'intégralité des pierres observées dans notre travail et dans les précédentes études, nous pouvons, à titre d'exemple, citer le cas du lion qui marche en avant, les oreilles rabattues et la gueule ouverte (pl. 16 à 19) .

Les habitudes d'observation et de représentation de l'animal sont donc très perceptibles sur les pierres. Le spectateur a l'impression de voir des sujets individualisés bien particuliers et non les représentants neutres d'une espèce animale (Sieveking, 1988). Mais il est notable que ce soin est également présent sur les autres représentations gravées. 


\section{Le soin apporté à toutes les gravures, y compris les plus abstraites}

À côté du style très réaliste et naturaliste qui domine largement à la Marche, nous avons en effet remarqué un mode de représentation caractérisé par un degré plus ou moins haut d'abstraction. Nous avons distingué trois niveaux de schématisation même si les limites entre ces niveaux sont assez floues. Tous témoignent cependant de l'attention que le graveur leur a portée.

Dans un premier mode de figuration, le graveur choisit de schématiser une représentation animale. Ceci se fait par la réalisation assez sommaire de la silhouette, laquelle ne va pas être élaborée de manière plus poussée, au moyen de détails figuratifs (fig. 11). Le résultat est la représentation d'un animal en général bien identifiable, mais simplifié au niveau graphique. Cette simplification ne peut cependant pas être considérée comme un indice du talent ou de l'habileté médiocre de l'artiste. Ces figures, bien que sommaires, sont en effet réalisées avec un nombre minimum de traits et, malgré cette réduction graphique, il est possible d'identifier la silhouette. De plus, on n'observe que rarement des corrections ou des reprises. Il est clair que seule une main expérimentée et habile a pu réaliser une telle gravure et que nous sommes loin d'une ouvre maladroite. Nous avons donc affaire ici à un choix motivé du graveur pour ce mode de représentation.

Dans un second mode de figuration, la schématisation est beaucoup plus poussée. Il s'agit de traits évoquant des lignes cervico-dorsales ou d'autres formes qui pourraient appartenir à des représentations figuratives. Les figures restent cependant incomplètes et leur attribution est ainsi incertaine. Nous connaissons des cas semblables dans d'autres sites magdaléniens comme à Limeuil en Dordogne, par exemple (Tosello, 2003). Or, il est notable que, bien qu'elles soient incomplètes, ces figures ne peuvent être confondues avec des gravures intégralement abstraites. Il y a donc bien une réflexion de la part du graveur qui l'amène à déterminer différents types de représentations.

Le troisième mode de figuration est formé par des traits gravés de manière intentionnelle, ce qui les distingue des traces d'usure. En effet, nous observons sur de nombreuses pierres des gravures complètement abstraites. Elles n'appartiennent pas à des figures animales incomplètes, et ne sont pas des esquisses. Nous avons évoqué le fait que ces traits peuvent soit occuper des surfaces entières, soit se limiter à certaines zones des surfaces gravées. Or, ces gravures possèdent souvent des types de tracés qui montrent aussi bien la volonté que le soin de l'artiste à les réaliser.

À côté de ces nombreux ensembles de traits abstraits, des motifs non figuratifs plus organisés (les signes) se distinguent: on peut citer, à titre d'exemple, une plaquette qui possède un signe en zigzag profondément incisé dans la surface, ou encore le galet gravé d'un motif abstrait complexe, en forme de quadrillage (Ja 709, fig. 20 et pl. 54).

\section{DES MOTIFS ARTISTIQUES AUX SENS MULTIPLES ET COMPLEXES}

La grande variété des modes de réalisation et de représentation nous montre que la présence de motivations différentes concernant la production des gravures s'impose. Il semble en effet que nous ne puissions pas interpréter tous les supports gravés de la même manière.

En conséquence, nous aimerions classer et proposer trois groupes différents de gravures, afin de discuter séparément de leur interprétation symbolique. Il s'agit des groupes animal, humain et abstrait.

\section{Des animaux souvent expressifs}

Sur les pierres déjà publiées, comme sur celles inédites qui forment le corpus principal de cette étude, nous avons remarqué la présence de motifs figuratifs animés de manière différente: allure des individus, association, élaboration des détails... Nous observons des scènes ou allures bien particulières répondant rarement à une «standardisation ». Il est certain que, face aux représentations qu'il produisait, l'homme ne maîtrisait pas seulement les techniques de gravure mais qu'il était, de plus, un excellent connaisseur de son environnement naturel. Il possédait par rapport à celui-ci une proximité mais aussi une capacité de recul, voire d'étonnement qui lui a permis de réaliser ses œuvres.

La proximité du Magdalénien avec la nature environnante peut être perçue par les statistiques dont nous disposons. Les gravures animales sont en effet les plus nombreuses dans le corpus de la Marche et les espèces représentées correspondent souvent à celles qu'il côtoyait quotidiennement. Le nombre de chevaux ou de cervidés et la précision avec laquelle ils sont souvent représentés en témoignent.

Mais les graveurs magdaléniens n'ont pas seulement réalisé des représentations d'animaux "familiers » (c'est- 
à-dire observés régulièrement). On peut aussi relever des gravures d'animaux rares ou aux postures particulières qui ont marqué les graveurs.

Rappelons, à titre d'exemple, la figuration du renne, présente sur une petite pierre mais effectuée de façon très détaillée (pl. 22 et 23). Nous avons ici affaire à une position très particulière, qui est probablement celle d'un animal blessé sur l'avant-train, pris juste au moment précédant l'écroulement. Même si aucune blessure n'est évidente dans la représentation, l'allure révèle la situation d'un animal grièvement blessé. En effet, habituellement, un cervidé ne prend pas une telle posture. Il ne s'agit donc pas d'une position statique mais d'un mouvement assez rapide fixé à un certain moment par la gravure. L'élaboration soignée de cette figure donne un aspect très vivant à la figuration et témoigne du fait que le graveur a certainement cherché à rendre la représentation la plus naturaliste possible.

Toujours est-il que, quels que soient l'espèce ou le comportement représentés, l'homme savait observer les animaux de manière précise et, ceci étant une étape capitale, les mémorisait pour pouvoir les représenter presque fidèlement dans un endroit où le modèle naturel est absent.

\section{Observation simple ou reflétant une interaction «homme-animal »?}

Mais réduire la production des gravures à une activité issue de la pure et simple observation ne paraît pas convenir à certaines représentations. Celles-ci en effet témoignent plutôt du rôle que l'homme lui-même a joué au sein de la nature. Les gravures peuvent alors être envisagées comme des transcriptions plus ou moins directes et visibles de l'interaction homme-nature.

Un des arguments les plus notables concerne, comme nous venons de le mentionner, la fréquence de la reproduction graphique de certaines espèces telles que les chevaux et les cervidés. Même si la valeur symbolique des animaux et la fréquence ou la qualité de leurs représentations ne peuvent pas être uniquement liées à l'importance numérique de telle ou telle espèce animale chassée, il semble pourtant que la chasse, activité primordiale, et l'observation active qui en découle, se soient montrées décisives dans la transcription graphique qui en a été faite.

Une pièce qui porte la figuration d'un loup, peut aussi étayer cette hypothèse (Ja 2, pl. 36 à 39). Là aussi, nous sommes en face d'un motif très individualisé et significatif d'un point de vue éthologique. L'animal semble être représenté dans une posture de soumission. Nous nous demandons par conséquent où l'homme a pu voir ce comportement. Il ne semble pas probable qu'il l'ait observé à l'intérieur d'une meute car, à l'état naturel, le loup n'a pas le même comportement en présence d'humains. La soumission est uniquement observable dans la vie au sein d'une meute qui n'est pas dérangée par une présence étrangère, telle celle de l'homme. Auprès de ce dernier, le loup sauvage va plutôt montrer de la peur (fuite) ou de l'agressivité (défense). Pour le représenter d'une manière si évidente, l'homme a cependant dû voir ce comportement à plusieurs reprises. C'est pour cela que nous sommes tentés de considérer cette représentation comme un indice indirect de l'apprivoisement, au moins partiel, du loup ou du chien sauvage. En observant un loup intégré dans un groupe humain, l'homme a certainement pu remarquer et étudier les comportements sociaux de cet animal dans ses détails et les représenter ensuite dans la gravure. Certes la question du rapprochement entre l'homme et le loup est actuellement encore controversée. Il semble cependant que le Magdalénien moyen constitue une époque de changement dans le rôle du loup pour l'homme. En effet, le site de la Garenne (Indre) a livré une ulna transformée en poinçon; or le travail de l'os de loup était, jusqu'à cette découverte, inconnu pour le Paléolithique supérieur. Comme beaucoup de carnivores, celui-ci tenait une place particulière dans la vie des hommes préhistoriques. Il était source de matières premières, par sa fourrure, et support de parure, par ses dents, même si la consommation systématique de sa chair ne peut être mise en évidence. L'utilisation de l'os du loup comme support de production d'outils apporte des précisions sur la relation entre celui-ci et les Magdaléniens. La gravure du loup de la Marche, quant à elle, pourrait faire partie de ces nouveaux indices montrant la proximité de l'homme et de cet animal, proximité qui s'exprime peut-être à cette époque en termes de changement. La question du moment de l'intégration des canidés dans les sociétés humaines peut donc être alimentée par cette représentation. En tout cas, il reste que ce n'est pas un hasard si l'homme a représenté l'animal dans cette position bien particulière. Le loup ou le chien soumis a certainement une signification profonde, même s'il ne peut s'agir que de la simple expression de l'autorité humaine sur le canidé. Faute d'autres représentations de ce type, il ne semble cependant pas avisé d'approfondir les hypothèses sur la signification de cette gravure.

Si l'observation, dont sont issues les représentations gravées, tient généralement compte de l'homme et de son interaction avec la nature, il semble plus adéquat de parler parfois de perception. 


\section{Observation ou perception?}

Celle-ci est notamment visible lorsque le Magdalénien a intégré dans ses représentations quelques imprécisions anatomiques. Ces imprécisions, voire ces « erreurs graphiques ", des gravures par rapport au modèle naturel ont déjà été mentionnées par L. Pales, par exemple pour les félins.

Nous nous sommes, quant à nous, interrogé sur les mammouths. À ce titre, le travail de recherche qui a été mené sur ces représentations en Dordogne, dans les grottes de Rouffignac, Font-de-Gaume, Bernifal et les Combarelles I et II, peut être cité.

Les mammouths figurés dans ces grottes sont, la plupart du temps, très bien dessinés, voire gravés. Certains éléments élaborés par les Préhistoriques sont en conformité avec le modèle naturel. Ceci est par exemple le cas pour les inclinaisons thoraco-lombaires qui sont en général de $45^{\circ}$ au maximum. On observe même parfois des reproductions de détails anatomiques assez réalistes tels que la trompe et l'œil. D'autres sont beaucoup plus rarement reproduits dans les grottes citées ci-dessus: c'est le cas des ongles des pattes, par exemple (Penvern, 2004). De même, d'autres imprécisions telles que l'hypertrophie, l'acrocéphalie, la courbure et la position des défenses peuvent être mentionnées. Certaines proportions anatomiques, comme le rapport de la tête et du corps, sont également déformées par rapport à la réalité. Prenant en compte tous ces éléments, l'auteur conclut qu'il ne s'agit pas vraiment de représentations naturalistes, comprises comme reproductions fidèles d'un modèle naturel.

Nous pouvons tout de même nous demander si cette différence entre les représentations et la réalité est intentionnelle ou issue d'un manque de connaissance de l'anatomie du mammouth. La question concernant la relation de l'homme et du mammouth est très controversée. En ce qui concerne le choix du mammouth comme gibier par exemple, certains spécialistes sont convaincus de la régularité de sa chasse comme élément de subsistance des groupes humains; d'autres pensent au contraire que le mammouth n'a pas été chassé systématiquement. Son importance symbolique est toutefois incontestablement démontrée par sa fréquence comme motif dans les représentations mobilières et pariétales ainsi que par le choix de cet animal comme motif de décorations d'armes et d'autres objets.

Pour ce qui est de notre propre point de vue, il nous semble que la représentation légèrement erronée des défenses du mammouth est un indice montrant que celle-ci s'est majoritairement faite d'après de simples observations répétées de ces animaux dans la nature. Elle ne dépend donc probablement pas d'une connaissance exacte de l'anatomie, notamment interne. Une autre analyse est également possible: il pourrait plutôt s'agir de la répétition d'un motif copié sur d'autres représentations de mammouths par des contemporains. Dans ce cas, nous aurions affaire à la prolifération graphique d'un motif ayant une symbolique interne détachée de l'animal réel.

Quelle que soit l'hypothèse retenue, il y a donc bien perception, c'est-à-dire observation et interprétation des animaux, dans certaines des représentations artistiques. Le prisme par lequel passait cette observation pouvait être lié aux contraintes de celle-ci (à savoir l'observation plus ou moins fréquente et aisée des animaux), mais aussi des caractéristiques culturelles appartenant à l'artiste (par exemple ce que représentait l'animal pour lui ou pour son groupe social).

Pour conclure avec ces représentations animales, on peut dire qu'il est absolument certain que l'homme entretenait un lien étroit avec son environnement et surtout avec le monde animal. L'importance du gibier comme moyen majeur de subsistance manifeste de manière nette le lien fort entre l'homme et les animaux. De manière tout aussi évidente, l'homme a choisi certaines espèces comme motif pour ses représentations. Ce choix semble souvent dépendre des animaux que l'homme connaissait bien et dont il observait l'environnement. Cependant le choix des espèces représentées et ignorées est sans doute lié aussi à des facteurs d'interaction "homme-nature " et à d'autres, plus spécifiquement culturels. Ceux-ci ont dû jouer un rôle non négligeable mais variable selon les régions et selon les époques.

\section{Les gravures humaines de la Marche}

Catégorie de représentations à part, les humains figurés à la Marche présentent eux aussi un caractère expressif divers et varié, mais ne semblent pas pouvoir être confondus avec les représentations animales. Contrairement au site de Gönnersdorf et à ses gravures de femmes stylisées, nous avons ici l'impression d'une individualisation quasi absolue de chaque représentation. Cependant, force est de constater qu'il n'existe pas, à la Marche, de gravure qui associe l'animal et l'homme dans un motif tel qu'on le connaît dans l'art mobilier de Laugerie-Basse (la «femme au renne ») ou d'Étiolles (Taborin, 2001).

Il est possible qu'ici nous ayons une manifestation de la séparation psychologique entre deux mondes, l'animal 


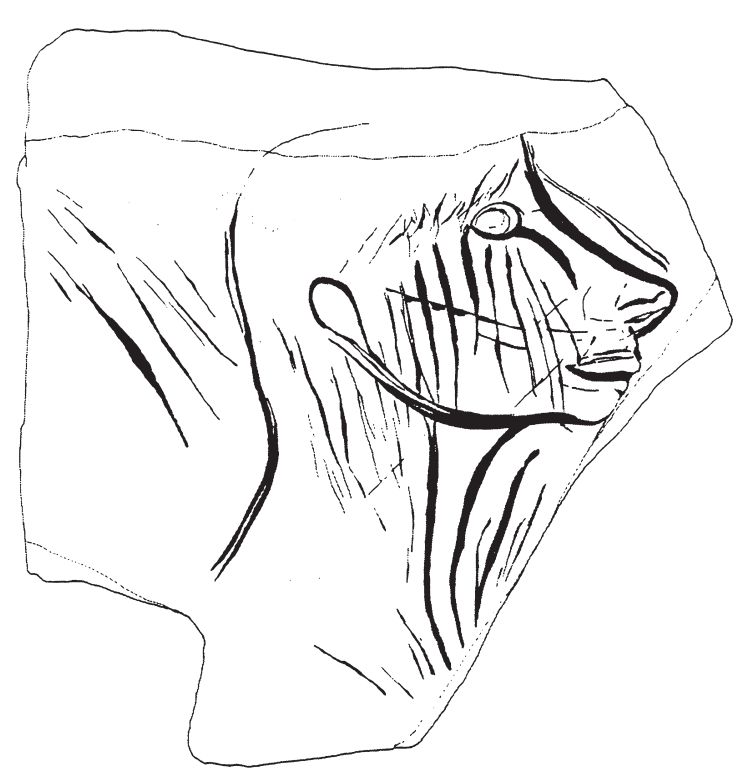

Fig. 52 - Gravure d'une " tête humaine bestialisée » découverte à la Marche (d'après Airvaux, 2001). Sans échelle.

et l'humain, encore que l'homme est lui-même toujours présent par l'acte de gravure des motifs. Il semble donc qu'il faille, pour l'interprétation de ces motifs, envisager quel regard le Magdalénien pouvait avoir sur lui-même, sur son groupe social et sur son existence.

Les gravures humaines dont nous disposons pour cette étude reflètent non pas une simple observation mais bien une interrogation des artistes magdaléniens sur le groupe humain. Celle-ci se manifeste d'abord par le nombre très élevé de ce type de représentations sur le site ( $c f$. statistiques), chose qui est d'ailleurs propre à la Marche. Mais, au-delà de la simple mention quantitative, c'est aussi le traitement de ces figures qui est en lui-même particulièrement éloquent.

On notera, à ce titre, que les gravures humaines représentent très régulièrement le visage. Or, celui-ci est bien le siège des émotions mais aussi de l'individualité et de l'humanité. Ces dernières notions semblent avoir été un des pôles d'interrogation des hommes préhistoriques dans le sens où les gravures de la Marche sont toutes précisément distinguées par des détails anatomiques ou de parure. De même, on a mentionné l'existence de dimorphisme, présent certes dans la célèbre plaquette de "l'homme bestialisé » mais aussi dans la caricature que l'on a découverte au cours de ce travail (fig. 52).

La question de l'individualité a pu être envisagée pour elle-même mais aussi en analysant le rapport entre « moi et l'autre ", c'est-à-dire la place de chaque homme au sein du groupe social. La plaquette LP 22, sur laquelle se trouvent deux représentations humaines est, de ce point de vue, particulièrement frappante (pl. 18 à 21). La mise en scène, d'abord, n'est pas anodine: deux visages opposés mais parfaitement cadrés sur la plaquette témoignent d'une séparation, qui est par ailleurs renforcée par les détails reportés. Les yeux, le nez, mais aussi la présence ou non de parure montrent que l'on a face à nous deux individus différents. Et, pourtant, cette distinction est en partie gommée par la liaison qui existe entre ces deux représentations: celle-ci se matérialise par des traits de chevelure qui se recoupent, de sorte qu'on a l'impression qu'il s'agit d'un Janus. Il semble donc qu'ici soit traitée la question de la proximité mais aussi de l'individualité de l'homme au sein d'un groupe.

Il semble que les gravures de la Marche soulèvent aussi d'autres aspects de l'existence humaine, comme son commencement ou son recommencement.

\section{Les gravures témoins des interrogations sur la vie}

Les thématiques de la sexualité, de la reproduction, voire de la naissance, sont récurrentes dans les représentations humaines de notre étude.

On citera par exemple le nombre imposant de représentations féminines dans le corpus humain de la Marche. Debout, assises avec les jambes étendues vers l'avant ou agenouillées, ces figures semblent être représentées dans des positions particulières. Il est cependant difficile d'attribuer ces allures à des comportements précis. Nous sommes surtout surpris par les figures assises avec les jambes étendues vers l'avant (fig. 17). Autant la position debout est fréquente et la position agenouillée également habituelle chez l'homme, autant la position assise avec un angle de $90^{\circ}$ entre tronc et jambes étendues ne lui est pas très commune. À notre connaissance, ces gravures sont d'ailleurs les seules figurations de cette sorte qui existent dans le Magdalénien. Toujours est-il que ces figures féminines sont souvent représentées avec un ventre très rebondi qui évoque une grossesse.

L'acte sexuel et/ou de reproduction est aussi maintes fois signifié dans les représentations associées. On peut citer comme exemple la plaquette associant une femme et un nouveau-né, ainsi que celle où l'on trouve un personnage féminin associé à une vulve (pl. 1, 2 et 28). Dans ces cas précis, un caractère narratif n'est pas à rejeter. Dans le premier, nous avons en effet une succession figurative thématique par superposition et, dans le deuxième, par juxta- 
position. Une femme tend ainsi les mains vers une vulve (pour en démontrer l'appartenance, l'importance?), tandis qu'une autre, enceinte, se trouve sous un nouveau-né, comme dans un enchaînement chronologique. Grâce à la microtopographie, on a même pu démontrer que ce bébé « évolue "sur la plaquette, puisqu'il possède deux représentations crâniennes, une l'identifiant comme nouveau-né au sens propre du terme (un enfant juste sorti du ventre de la mère) et une autre qui témoigne de l'évolution morphologique de cette partie du corps, quelque temps après la naissance. Ce type de gravures était peut-être accompagné d'un récit ou d'une histoire. Toute argumentation plus poussée reste cependant gratuite, faute d'éléments démontrables.

Il n'en reste pas moins que les motifs mêmes ont une expression symbolique forte qui est due à l'association des formes et des thèmes, et que cette expression reflète certainement une interrogation sur les processus naturels qui aboutissent à la mise au monde d'un nouvel homme.

Ces figures humaines ont donc une signification propre et importante qui témoigne de ce qu'observait, ressentait et vivait l'homme magdalénien. Cependant nous avons également remarqué, dans les gravures de la Marche, l'abondance des motifs à caractère non figuratif. Leur nombre important rend nécessaire de les inclure dans le corpus iconographique et de discuter plus en détail leur rôle et leur importance graphique.

\section{Des abstractions au sens complexe et diversifié}

Le domaine de l'abstraction joue en effet à la Marche un rôle important, au côté des figurations animales et humaines. Nous avons déjà montré que les motifs abstraits se présentent sous des formes et dans des contextes divers. L'interprétation doit par conséquent s'effectuer également sur des niveaux différents.

\section{La schématisation des représentations figuratives: un statut intermédiaire difficile à définir}

En ce qui concerne les figures animales simplifiées ou stylisées, nous n'avons pas assez d'échantillons à notre disposition pour déterminer s'il s'agit de la main d'un ou de plusieurs artistes. En tout cas, la démarche graphique, plus sommaire, n'est pas la même que celle liée à l'élaboration détaillée des figures naturalistes.

Les figures animales sont en effet parfois réduites à des lignes cervico-dorsales, c'est-à-dire des lignes ondulées dont le geste rappelle la réalisation de certaines parties des figures animales. Leur détermination est cependant souvent impossible. Le problème se pose aussi pour les "yeux isolés ». Ces formes ovales, arrondies ou en forme d'amande, évoquent effectivement des yeux d'animaux ou d'humains. L'absence de tout autre élément anatomique ne permet cependant pas de concevoir ces gravures comme des motifs figuratifs équivalant à des figurations animales plus complètes: en effet, il pourrait également s'agir de formes abstraites.

Malgré ces incertitudes, deux hypothèses interprétatives peuvent être énoncées.

La première est que les graveurs magdaléniens possédaient une convention consciente ou non des modes de représentation de certains éléments graphiques et que celle-ci déterminait en fin de compte leur façon de dessiner et de graver. En ce cas, ces formes intermédiaires schématisées contenaient un sens patent pour le Magdalénien mais aujourd'hui perdu pour nous. La seconde hypothèse interprète ces gravures comme une sorte «d'automatisme » de représentation, c'est-à-dire qu'elles seraient le résultat d'un geste habituel, presque "mécanique ». Il pourrait alors s'agir du commencement d'une figure par la suite abandonnée, d'un entraînement, ou plus simplement d'un acte de gravure sans but représentatif précis.

Malheureusement, il ne semble pas possible, en l'état actuel des recherches, de pousser plus avant l'interprétation de ce type de gravures. Cela ne doit pas nous empêcher cependant d'envisager celle des signes abstraits.

\section{INTERPRÉTATIONS DES SIGNES ABSTRAITS}

Les signes abstraits à composition géométrique apparaissent généralement sous des formes simples telles que des rangées de traits parallèles ou d'autres formes répétées. Ils sont sans aucun doute gravés de manière intentionnelle. Leur degré d'élaboration est variable. Ainsi avons-nous identifié de simples rangées de traits courts et parallèles mais également des zigzags. Une autre gravure de ce type est encore plus complexe: il s'agit d'un quadrillage qui divise un galet en plusieurs secteurs (pl. 54).

Il est bien entendu tentant d'essayer de déterminer la ou les relations qu'entretiennent les gravures figuratives et ces signes. À ce titre, seuls les traits parallèles semblent, sur certaines pièces, nous fournir des indices. En effet, ou bien, comme c'est le cas pour une pierre portant la figuration d'un renne, les traits « rayent » la figuration (pl. 22 et 23), ou bien c'est l'espace resté libre, en face d'un profil humain, qui a été rempli avec des traits parallèles. Dans le premier 
exemple, il est possible que nous soyons en présence d'une destruction volontaire de la représentation. Dans le second, les traits n'affectent pas directement le motif mais sont en relation avec lui. D'autres exemples manifestent des cas semblables. Ainsi en est-il pour la plaquette portant le visage d'un homme barbu vu de face (fig. 16). La tête est antérieure aux traits indéterminés et ceux-ci semblent dissimuler le visage. Ceci peut être interprété comme un geste symbolique du graveur. S'agit-il ici d'un effacement intentionnel de la tête dans un sens complètement destructeur ou le graveur a-t-il simplement voulu dissimuler le visage? Sans pouvoir répondre à cette question, on peut du moins soulever ces hypothèses. De même, une autre plaquette montre une association d'un autre type, mais de discours comparable. Il s'agit de la représentation d'une tête humaine associée à deux tracés indéterminés (pl. 55 et 56). Son interprétation semble être plus saisissable et accessible: la tête humaine est en effet barrée par deux traits qui semblent être le résultat d'un effacement intentionnel par le graveur lui-même. La morphologie des traits indique de fait que c'est probablement le même outil qui a gravé puis rayé cette tête. On peut certes se demander si c'était le même artiste. En tout cas, l'usure assez rapide d'un silex lors de la gravure ne permet pas de concevoir qu'un grand décalage ait existé entre la réalisation de la tête et celle des deux traits.

On ne peut pourtant pas tenir compte que des signes abstraits en corrélation avec des représentations figuratives dans le cadre de cette interprétation. Ce serait omettre en effet tous ceux qui sont seuls et qui représentent une catégorie d'expression graphique particulière. Comment dégager le sens de ces formes? Il semble qu'il faille en réalité en envisager de multiples.

Dans les cas où il y a bien rapport entre l'abstrait et le figuratif, les exemples détaillés tendent à nous faire penser que le premier est mis « au service » du second. Les tracés ont alors une fonction de « dissimulation » ou de « destruction".

En revanche, lorsque les signes sont isolés et qu'ils ont une forme plus ou moins complexe, on peut alors dire que c'est celle-ci qui a intéressé le graveur. Il ne s'agit pas en effet d'une transcription de la nature observée et vécue. Plusieurs hypothèses peuvent être ici valorisées: soit ces signes ont comporté un sens particulier aujourd'hui perdu puisque la convention est oubliée; soit le graphisme obtenu est le seul effet recherché; soit enfin, pour les signes les plus simples notamment, c'est l'acte de gravure lui-même qui a peut-être importé. Là encore, la diversité des interprétations envisageables et leur possible combinaison, ne nous permettent pas d'aller plus loin dans l'étude du sens de ces figures.

$\mathrm{Au}$ terme de cette analyse sur les gravures, plusieurs points importants peuvent donc être dégagés, même si des zones d'ombre subsistent. Ceux-ci concernent essentiellement la place et le statut du graveur magdalénien et de la gravure à partir de l'exemple de la Marche.

En premier lieu, on peut dire que ce graveur est un « professionnel » : le soin et la maîtrise dont il a, à multiples reprises, fait preuve en témoignent. Reste à déterminer son «visage » : s'agissait-il en effet d'un individu spécialisé au sein du groupe ou bien la gravure faisait-elle l'objet d'un apprentissage systématique et méticuleux, du fait de l'importance qu'on lui conférait? Pour l'instant aucune de ces deux hypothèses ne peut vraiment être validée.

En second lieu, le graveur magdalénien présente un reflet de ce qu'a pu être l'homme préhistorique de cette époque. Les gravures et l'acte dont elles sont issues supposent un homme proche de la nature mais aussi de la matière. "Observation ", « précision ", « choix raisonné " sont autant de mots-clés qui viennent confirmer cet état de fait. Il n'en reste pas moins que cet homme s'insère d'abord et avant tout dans un groupe social et culturel. L'étude détaillée des gravures, de leurs significations, voire de leurs conditions d'élaboration, nous amène à envisager cette question, à notre sens importante: quelle était la part de «liberté »du graveur, notamment dans le choix du motif? La répétition des représentations et de leurs formes (à un point tel que l'on a pu élaborer des statistiques et des typologies), les imprécisions relevées (dont on a dit qu'elles provenaient peut-être d'une perception particulière), tout cela va dans le sens de l'existence d'une ou plusieurs conventions graphiques. Les gravures pourraient alors être en partie vues comme un outil de communication au sein du groupe social, comme une variation sur un même message. Et pourtant, il semble qu'il faille nuancer cette hypothèse car il existe, au sein du corpus de la Marche, des figures rares et/ou traitées de manière originale, ainsi que des signes abstraits complexes et isolés. N'avons-nous pas, avec ces exemples, l'expression d'un véritable artiste, en ce sens qu'il a su faire preuve de talent, de curiosité et d'une faculté à faire passer dans ses œuvres son interrogation sur le monde qui l'entoure, sur les autres, voire sur lui-même? En ce cas, les gravures posséderaient une fonction artistique et symbolique forte, qui est de révéler ce que l'homme perçoit et ressent.

On voit bien par conséquent que le statut de la gravure est intrinsèquement lié à celui du graveur, et que celui-ci a pu être multiple. Il n'en reste pas moins que par son 
importance numérique et par le soin qui lui a très souvent été apporté, cette gravure avait une place notable au sein de la vie des Magdaléniens. Mais là encore s'exprime la complexité de ce domaine: comment, si cette forme d'art tenait un rang prestigieux aux yeux des hommes de la Marche, expliquer les palimpsestes et les cas de destruction graphique volontaire des motifs? Et surtout comment envisager le lien entre la gravure et la réutilisation des pierres à d'autres fins? Il semble qu'il faille aborder maintenant plus précisément cette seconde interrogation.

\section{UN EMPLOI DES PIERRES À DES FINS QUOTIDIENNES}

Lors de notre analyse, nous avons démontré que les graveurs de la Marche maîtrisaient parfaitement les techniques de la gravure et connaissaient les contraintes des matériaux qu'ils utilisaient. Ainsi ont-ils adapté leurs outils et leurs gestes aux propriétés mécaniques et aux particularités morphologiques des supports. Il semble évident qu'une grande partie de ces derniers ont été en premier lieu choisis et employés en tant que supports de gravures. Toutefois, nos études ont montré que la fonction des pierres ne s'arrête pas à cet unique emploi mais qu'elles en ont eu d'autres au sein du gisement. Trois phénomènes que nous avons, à maintes reprises, relevés méritent d'être à ce titre soulignés: la fracturation, la rubéfaction et les traces d'utilisation. Nous allons donc maintenant revenir sur ces trois domaines, puis finalement tenter d'évaluer les rapports qu'entretiennent les traces d'utilisation des supports avec celles des expressions graphiques.

\section{LES FRACTURES DES SUPPORTS}

Nous avons remarqué, lors de notre étude, l'énorme taux de fractures des supports gravés. Ceci n'est pas forcément une spécificité du site de la Marche. En effet, d'autres sites montrent également l'existence de plaquettes gravées fracturées, lesquelles, la plupart du temps, sont éparpillées dans les gisements de manière plus ou moins organisée. Nous pensons en particulier aux sites d'Enlène, de Limeuil et de Gönnersdorf (Bosinski, Fischer, 1974; Bosinski, 1990; Clottes, 1999; Tosello, 2003).

La fracturation atteint aussi bien les plaquettes assez fines que les grandes dalles très épaisses. Si, pour les premières, une fracturation par altération mécanique ou piétinement semble possible, cela semble très improbable pour les grands supports. Encore peut-on penser à un éclatement par chocs thermiques. C'est dans ce contexte qu'a été suggérée, pour le site de Limeuil, l'idée de la fracture intentionnelle par l'homme: celui-ci aurait lancé les supports d'une certaine hauteur pour les réduire en blocs maniables (Tosello, 2003). Cette procédure ne laisse pas de traces de mise en forme particulières, telles que des encoches de négatifs d'enlèvement ou des zones d'écrasement dues à des impacts ciblés. De ce fait, une fracturation par cette technique aurait pu se dérouler lors de la manipulation et l'emploi des plaques à la Marche. Sur certaines d'entre elles, nous avons cependant également identifié des traces directes de mise en forme ou de fracturation sous forme d'encoches. Ceci montre, du moins en ce qui concerne les cas où nous avons détecté ces stigmates, que nous sommes en face d'une fracturation par percussion ciblée. Les Magdaléniens ont, de toute évidence, fracturé et taillé certains blocs calcaires à l'intérieur du gisement, parfois sans se préoccuper des gravures qui s'y trouvaient. Il existe effectivement des pierres où la mise en forme a partiellement emporté les gravures. À l'inverse, dans aucun des cas, une relation directe entre mise en forme et gravures n'a pu être démontrée. Nous pouvons en déduire que la mise en forme ou la fracturation des plaquettes ne paraissent pas avoir été employées pour les préparer à la gravure.

Il semble également que les pierres aient fonctionné comme éléments d'aménagement dans les structures de combustion. C'est pour cela qu'il convient de nous attarder sur la rubéfaction.

\section{LES TRACES DE RUBÉFACTION SUR LES PIERRES}

L'étude des pierres a montré qu'un certain nombre d'entre elles porte des traces de rubéfaction. Il semble clair que ces pièces ont subi l'action d'une chauffe assez importante pour garder les traces de ces impacts thermiques. En effet, les premiers fouilleurs de la Marche ont mentionné deux foyers dans la couche archéologique, qui se distinguaient par leur concentration en cendres mais également par leurs pierres calcaires. Ce sont sans doute ces dernières qui ont servi pour aménager ces structures. Des comparaisons avec d'autres sites, Gönnersdorf par exemple, montrent que cet emploi de pierres pour l'aménagement de foyers est relativement fréquent. De même, au sein de ce site et plus précisément dans les structures de combustion elles-mêmes, se trouvaient aussi bien des pierres gravées que non gravées. À partir de cette com- 
paraison, des informations dont nous disposons et de nos propres observations, nous pouvons affirmer qu'une telle utilisation des pierres calcaires, y compris gravées, a sans doute aussi existé à la Marche. Lorsque l'on parle d'aménagement, il semble donc qu'il faille envisager un emploi des pierres calcaires comme éléments structurants, soit pour les habitations elles-mêmes, soit pour la construction d'objets utiles à la vie quotidienne, comme les foyers. Mais, outre des traces de fracturation et de rubéfaction, nous avons pu identifier des traces d'usure et d'utilisation distinctes qui témoignent également de l'emploi des pierres pour d'autres usages que la gravure.

\section{LES TRACES D'UTILISATION SUR LES PIERRES}

La présence de traces d'usure et d'utilisation a été à plusieurs reprises détectée sur les pierres de la Marche.

Notre étude a montré qu'il s'agit souvent d'éraflures ou d'impacts légers sous forme de rayures qui pourraient provenir de l'utilisation des pièces comme supports de travail, par exemple pour la découpe de matières organiques ou pour la perforation.

À cela s'ajoute la présence de surfaces usées. Il est probable que cette usure, qui ne se trouve pas sur toutes les pièces et qui, quand elle s'y trouve, possède une intensité variable selon les supports, ait été provoquée par piétinement. Ce dernier a engendré un frottement répété des surfaces, frottement renforcé par la présence d'un abrasif; il est, par ailleurs, envisageable que celui-ci soit simplement constitué des sédiments fins naturellement présents dans l'habitat.

Reste à mentionner le phénomène des lustres légers qui ont été repérés sur certaines pierres. Leur aspect lisse plus ou moins marqué peut s'expliquer par leur manipulation répétée. En effet, lors de cette opération, certains endroits de l'objet sont plus exposés au contact avec les mains que d'autres. Ainsi trouvons-nous souvent ce genre de lustres ou de polis sur les bords des pierres gravées.

Il est enfin intéressant de noter qu'une seule et même pierre peut porter plusieurs traces d'utilisations différentes, ce qui témoigne de la circulation et du changement fréquent de fonction de l'objet dans le site.

Chaque support possède ainsi une histoire individuelle riche et l'ensemble des pierres reflète la diversité, parfois complexe à appréhender, de leur emploi au sein du gisement. C'est finalement dans cette histoire changeante que se placent les gravures.

\section{PLACE DE LA VIE ARTISTIQUE DANS LA VIE QUOTIDIENNE}

En ce qui concerne la relation entre les gravures et les autres traces d'utilisation sur les pierres, nous avons vu qu'en termes de chronologie relative il existe des gravures antérieures aux usures et, inversement, des surfaces usées qui ont été regravées. Il en est de même pour les autres traces, telles que la fracturation et la rubéfaction. Ceci montre à quel point les gravures se trouvent dans une interrelation avec les traces qui témoignent d'un emploi plus «banal » (au sens d'usuel) des supports dans le site.

$\mathrm{Au}$ terme de ce second point, nous pouvons donc nous interroger plus avant sur le statut et l'importance accordés aux gravures pour les hommes du Magdalénien. Nous sommes forcés de constater que la réponse à ces questions est certainement multiple.

Certaines figurations sont véritablement dissimulées sous des entrecroisements de traits gravés volontairement et/ou usuels. Il semble évident qu'à un moment donné ces gravures ont perdu de leur importance. De ce fait, il convient de se demander si, dans ces cas-là, ce n'était pas l'acte de gravure lui-même et/ou le contexte dans lequel il avait été effectué qui étaient au centre de l'intérêt.

Mais, comme nous venons de le souligner, nous avons certainement affaire à une coexistence de modèles explicatifs et donc à la simultanéité de plusieurs hypothèses d'interprétation possibles des gravures. Ainsi existe-il des pierres gravées sur lesquelles c'est sans doute le motif même qui a été recherché et conservé. C'est le cas, par exemple, de la gravure du lion sur une plaque de taille plus imposante, gravure qui est par ailleurs curieusement isolée (en ceci qu'elle n'est pas ou peu recoupée par d'autres traits). Ici, c'est bien le produit final de l'acte de gravure qui a ainsi été valorisé.

Pour conclure sur l'interprétation de ces pierres gravées du site de la Marche, plusieurs points peuvent être abordés.

D'abord, pour ce qui est de leur utilisation usuelle, on peut dire que celle-ci a été omniprésente. Les pierres ont pu être employées directement ou non par les Magdaléniens: directement, en tant que supports mobiles d'activités quotidiennes comme la découpe ou la perforation; indirectement, en tant qu'éléments structurels de l'habitat lui-même (pierre de calage, dallage, etc.) ou d'aménagements dans cet habitat («mobilier», foyer, etc.). 
En ce qui concerne l'interrelation entre l'activité de la gravure et les autres activités dans le gisement, nous pouvons retenir que nous sommes apparemment face à un mélange total de ces activités culturelles, alimentaires et artisanales. La gravure sur pierres mobiles et sur blocs se place donc essentiellement dans le contexte du fonctionnement d'un site d'habitat. De fait, les pierres gravées sont un témoin important de la place des activités artistiques au sein des lieux de vie des Magdaléniens de la Marche. Il n'en reste pas moins que cette interprétation peut être nuancée par certains cas particuliers, qui montrent que la compréhension du mode d'existence magdalénien n'est pas encore achevée et que celui-ci est particulièrement complexe: il s'agit de gravures qui semblent avoir profité d'un traitement et/ou d'un statut spécifiques. Le premier exemple est celui, déjà mentionné, du lion. Le second fait appel aux témoignages des premiers fouilleurs et ne peut malheureusement pas être confirmé ou infirmé: on pense ici au cas de la « tête bestialisée » qui, non seulement, a été gravée au moyen de tracés profonds peu utilisés par les hommes de la Marche, mais en plus aurait été placée au sein du site dans une structure lithique particulière qui fait penser à un dépôt. Au terme de cette étude, ce sont donc les mots « complexité » et «multiplicité » qui semblent définir au mieux les pierres du site: multiplicité des natures, des rôles et des statuts de ces supports lithiques qui rend leur lecture et leur compréhension aujourd'hui difficiles.

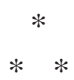

"Ce travail est dédié à la mémoire des graveurs non conformistes de la Marche » (Pales, Tassin de Saint-Péreuse, 1976).

C'est avec cette phrase, assez étonnante à première vue, que L. Pales a ouvert son ouvrage sur les représentations humaines de la Marche en 1976. Elle exprime l'étonnement de l'auteur face à la qualité des gravures, à l'habileté, à la créativité et au sens de l'observation dont ont su faire preuve les graveurs magdaléniens.

$\mathrm{Au}$ terme de cette étude, nous pouvons nous-mêmes réaffirmer sans peine l'intérêt artistique des pierres gravées de la Marche, tout comme nous pouvons maintenant affirmer l'importance de ces supports au sein de la vie quotidienne dans ce site d'habitat. En quoi, finalement, ce site est-il un reflet de ce qu'a pu être la vie au Magdalénien? Comment, cependant, s'insère-t-il dans son propre contexte chronoculturel? Sans être exhaustif, ce travail permet d'apporter quelques éléments de réponse à ces questions.
La Marche est ainsi, comme beaucoup d'autres sites, un lieu de vie au sein duquel les hommes préhistoriques se sont livrés à des activités artistiques. Et pourtant, ce qui reste pour le moins curieux, c'est la densité des gravures trouvées: tout se passe comme si, dans cet endroit, les Magdaléniens avaient entretenu plus qu'ailleurs une étroite proximité entre la vie quotidienne, qui permet la subsistance, et la vie culturelle, qui permet, au sens philosophique du terme, l'existence.

En effet, la richesse et l'originalité de l'iconographie mettent en relief l'importance accordée à une activité qui n'a pas en soi de finalité vitale, mais qui permet apparemment à l'homme de fixer l'univers dans lequel il vit et de réfléchir à la place qu'il tient au sein de celui-ci. la Marche a ceci de commun avec d'autres sites qu'elle comporte de multiples représentations d'animaux habituels, mais aussi d'autres plus rares. Celles-ci, comme le loup ou l'éléphant des mers, témoignent d'un choix particulier qui fait l'originalité du site et rouvre, pour le chercheur, l'interrogation sur le sens des motifs. Cette originalité se retrouve dans le nombre et le traitement artistique des représentations humaines et, parfois même, des signes abstraits. Les représentations humaines sont, par ailleurs, une particularité de la Marche: celle-ci renforce l'idée d'une démarche mentale et culturelle particulièrement vive dans ce site.

Reste à noter que cette démarche est servie par des techniques de gravure diversifiées mais qui s'intègrent quand même dans un corpus global de « conventions ». Là encore s'expriment à la fois la proximité et la spécificité de la Marche en comparaison avec d'autres sites magdaléniens. En effet, l'habileté des hommes de cette époque a été à maintes reprises observée: leurs gravures témoignent très souvent d'un talent qui fait d'eux des artistes à part entière. Et pourtant, on ne peut que relever l'adaptation des Magdaléniens de la Marche à ces supports particuliers que sont les calcaires bathoniens et kimméridgiens. La maîtrise de leurs contraintes, tout comme la rareté de l'emploi d'autres matériaux, mettent en lumière ce choix, mais un choix en partie conditionné par le site et ses spécificités: ainsi l'alliance entre les particularités propres au lieu et l'adaptation des hommes qui y résidaient ont fait de l'art de la Marche un art original, sur support calcaire, comme Gönnersdorf l'est par le biais du schiste.

C'est donc, au final, l'image d'un site à la fois original et caractéristique du Magdalénien moyen qui se dresse ici. Il est cependant clair que, bien que notre échantillon de travail soit parfaitement représentatif, la complexité de la Marche n'est pas épuisée. Nous voudrions, à ce titre, 
proposer un certain nombre de pistes de recherche que nous envisageons pour la suite de l'étude.

La découverte de figures nouvelles est, certes, importante et d'un grand intérêt pour le travail sur les représentations du Paléolithique supérieur. Il faut cependant élargir, comme nous l'avons tenté ici, le champ d'investigation.

Ainsi, une étude complète concernant la technique de gravure doit certainement être menée sur d'autres supports. Cela serait d'un grand intérêt pour l'élargissement des connaissances sur les pierres gravées. Une telle étude pourrait, par ailleurs, être enrichie par des approches d'archéologie expérimentale: cette dernière pourrait livrer des informations précieuses sur la démarche artistique et technique.

Ce dernier point nous amène à une autre question, celle des outils utilisés. L'industrie lithique de la Marche est, en effet, très riche et peu de travaux ont été consacrés à cet ensemble. Une étude systématique pourrait donc nous livrer des informations nouvelles sur les outils susceptibles d'avoir été utilisés pour la gravure.

De même, l'étude globale du site de la Marche et de ses vestiges archéologiques serait importante et d'un grand intérêt. Une telle étude devrait notamment inclure celle des restes fauniques. Les résultats d'un tel travail pourraient être utilisés pour envisager à nouveau le rôle des animaux dans le site et leurs liens avec les gravures.

En effet, nous avons pu démontrer ici que les pierres gravées constituent des supports complexes, combinant, au sein du gisement, des rôles et des statuts différents. Une mise en contexte de ces objets avec l'intégralité des vestiges est donc indispensable pour une compréhension plus précise encore de leurs fonctions et de leur histoire. Celle-ci pourrait également être éclairée par une nouvelle comparaison qui concernerait le site de la Garma, en Espagne.

Il est enfin clair que les moyens d'investigation utilisés dans le cadre de ce travail pourraient être réemployés. Ceux-ci ont, en effet, montré qu'ils étaient d'un grand intérêt pour la recherche préhistorique, intérêt que nous voudrions, pour finir, resituer.

\section{TECHNIQUES ET MOYENS D'INVESTIGATION}

Le MEB et le microrugosimètre offrent tous les deux des propriétés techniques particulières et sont des moyens d'étude à la fois très précis et complémentaires.

Le MEB sert parfaitement à étudier les pigments et les sédiments sur un plan morphologique et chimique.
Actuellement, il est également utilisé pour l'analyse des gravures sur les objets préhistoriques.

Cependant, dans le cadre de ce travail, nous avons plutôt tenté de mettre en ouvre une nouvelle technique qui est celle de la microtopographie. Bien qu'il existe plusieurs techniques de saisies tridimensionnelles de surfaces, celle de la microtopographie confocale à champ étendu s'est avérée être particulièrement adaptée à notre étude. Comme pour l'imagerie au MEB, nous avons montré que celle-ci permet de travailler sur des détails microscopiques des surfaces gravées et d'en tirer une panoplie d'informations sur la nature des traits et la technique de gravure. Mais la microtopographie dispose pour cela d'avantages majeurs. D’abord, cette approche ne nécessite aucun contact avec l'objet archéologique; il est donc possible de travailler directement sur l'original. La saisie ne nécessite pas non plus de conditions particulières (vide ou vide partiel) : il n'y a pas, a priori, de contraintes de taille de l'échantillon. De plus, ces saisies produisent une image tridimensionnelle à haute résolution. Ainsi avons-nous accès aux trois dimensions de la surface à chaque endroit de la zone relevée. Les données ont pu être analysées de manière qualitative et quantitative selon des protocoles divers issus des questionnements. Nous avons de fait présenté deux types d'étude bien distincts, notamment par l'échelle d'observation.

Le premier concernait l'analyse de gravures et des traces d'utilisation sur les pierres gravées. Les données tridimensionnelles offrent la possibilité de mesurer les dimensions exactes des incisions et stries au centième voire au millième de millimètre près; elles permettent aussi d'observer l'évolution des profils, l'état de conservation des surfaces et les formes des parcours de tracés. De plus, les images synthétiques produites avec ces données nous ont permis d'identifier des détails figuratifs qui n'étaient pas clairs lors du relevé des gravures.

Le second type d'étude relevait du domaine de la tribologie, c'est-à-dire de l'analyse des microsurfaces $\left(<1 \mathrm{~mm}^{2}\right)$. Celles-ci nécessitent des mesures de haute précision. Dans ce cadre, nous avons pu évaluer l'impact du frottement de l'outil sur une surface et ainsi envisager la distinction entre des traits naturels et anthropiques. La tribologie a également été utilisée pour distinguer la nature des supports rencontrés. Ainsi l'analyse de la rugosité des surfaces calcaires, combinée à des observations macroscopiques et à la loupe binoculaire, nous a-t-elle permis de caractériser certains des supports présents à la Marche.

Une telle utilisation du microrugosimètre a donc aidé à mieux appréhender la nature des supports, la morphologie 
des traces anthropiques et l'histoire des pierres; l'interprétation de leur rôle au sein d'un site préhistorique est dès lors devenue possible.

L'analyse microtopographique s'avère donc être une démarche intéressante pour toute étude de pièces archéologiques. Bien sûr, il s'agit d'un moyen d'investigation qui ne peut être mis en œuvre qu'après une réflexion préalable et approfondie sur les supports d'étude. Il faut enfin savoir que le domaine de l'imagerie et de la saisie tridimensionnelle de surfaces est actuellement en cours de développement. Les techniques s'optimisent très rapidement pour gagner en rapidité, précision et étendue du champ de saisie. Il existe d'ores et déjà des techniques qui permettent d'établir le relevé d'une surface en quelques secondes, avec une résolution de moins de $500 \mu \mathrm{m}$. De plus, la taille et le poids des machines diminuent constamment. Il est ainsi très probable que la microtopographie pourra bientôt être appliquée dans les grottes ornées pour aider au déchiffrement et à l'analyse des supports rocheux. Les études de l'art mobilier, pierres et os gravés par exemple, sont également concernées par ce développement technique et une panoplie d'analyses, dans la lignée de celles que nous avons menées à titre d'expérimentation, seraient envisageables pour améliorer les connaissances des vestiges et assurer au mieux leur protection et leur valorisation.

Pour conclure, on peut dire que ce travail sur les pierres gravées inédites de la Marche a pu montrer, qu'en mettant en œuvre une approche méthodologique appropriée et en utilisant des moyens techniques adaptés, on peut en grande partie maîtriser la complexité des supports. Ces derniers deviennent, pour le chercheur, non seulement plus « visibles » mais aussi plus « lisibles » (c'est-à-dire compréhensibles).

Les nouvelles images issues du MEB et du microrugosimètre permettent d'appréhender ou, mieux encore, de « décoder » les pierres gravées. Elles nous permettent ainsi d'avoir une bonne image de ce que ces dernières sont aujourd'hui et de ce qu'elles représentaient au Magdalénien. 


\section{BIBLIOGRAPHIE}

\section{AIRVAUX J.}

1998 : " Découverte d’une grotte ornée, le réseau Guy-Martin à Lussac-les-Châteaux, Vienne et application d'une méthodologie structurale pour l'étude de l'art préhistorique ", L'Anthropologie, 102, 4, p. 495-521.

2001 : L’Art préhistorique du Poitou-Charentes : sculptures et gravures des temps glaciaires, Paris, Maison des Roches, 224 p.

2002 : « Méthodes de relevés des gravures paléolithiques : méthodologie structurale pour l'étude de l'art préhistorique et interprétation des significations ", in MiskOVSKY J.-C. (DIR.), Géologie de la Préhistoire, Perpignan, éd. Géopré-Presses universitaires de Perpignan, p. 425-446.

AIRVAUX J., FOURCHER P. avec la collab. de MAGNANT D., MARANDET F., BERNARD J. 1991 : « Les techniques informatiques du traitement de l'image appliqué à l'étude des gravures paléolithiques ", Paléo, 3, p. 139-147.

\section{AirvauX J., MÉlard N.}

2007 : « Regard sur les représentations paléolithiques de Pinnipèdes au Paléolithique supérieur : les phoques de la Marche et du réseau Guy-Martin (Lussac-les-Châteaux, Vienne) ", Préhistoire du Sud-Ouest, 2, p. 135-150.

\section{Airvaux J., Pradel L.}

1984 : « Gravure d'une tête humaine dans le Magdalénien III de La Marche, commune de Lussac-les-Châteaux (Vienne) ", Bulletin de la Société préhistorique française, 81, 7, p. 212-215.

\section{AUZANNE I., FUentes O.}

2003 : " Le "sorcier" du Roc aux Sorciers, Angles-sur-l'Anglin : nouveaux éléments d'analyse ", Antiquités nationales, 35, p. 41-54.

BOSINSKI G.

1979 : Die Ausgrabungen in Gönnersdorf 19681976 und die Siedlungsbefunde der Grabung 1968, Wiesbaden, Franz Steiner Verlag, $220 \mathrm{p}$.

1982 : Die Kunst der Eiszeit in Deutschland und in der Schweiz, Bonn, Habelt, 92 p.

1987 : Die große Zeit der Eiszeitjäger. Europa zwischen 40000 und $10000 v$. Chr., Mainz, RGZM (coll. Jahrbuch des römisch-germanischen Zentralmuseums, 34), 139 p.
1990 : Homo sapiens : l'histoire des chasseurs du Paléolithique supérieur en Europe $(40$ 00010000 avant J.-C.), Paris, Errance, 281 p.

1994 : «Die Gravierungen des MagdalenienFundplatzes Andernach-Martinsberg ", Jahrbuch des römisch-germanischen Zentralmuseums Mainz, 41, 1, p. 19-58.

1996 : « Die Nashorndarstellungen von Gönnersdorf ", in CAMPEN I. (DIR.), Spuren der Jagd : die Jagd nach Spuren. Festschrift für H. Müller-Beck, Tübingen (coll. Tübinger Monographien zur Urgeschichte, 11), p. 177-189.

\section{BOSINSKI G., BOSINSKI H.}

1991 : « Robbendarstellungen von Gönnersdorf ", in ATKAS A., BOENIGK P. (DIR.), Sonderveröffentlichung Geologisches Institut der Universität zu Köln, Köln (coll. Festschrift für Karl Brunnacker, 82), p. 89-103.

2006 : « Cuervo, rana y tortuga en Gönnersdorf : animales representados raras veces, que han sido dibujados perfectamente ", Munibe, Homenaje a Jesús Altuna, 57, 3, 2005-2006, p. 135-141.

\section{BoSINSKI G., D'ERrico F., SCHILler P.}

2001 : Die gravierten Frauendarstellungen von Gönnersdorf : der Magdalenien Fundplat Gönnersdorf, 8, Mainz, Franz Steiner Verlag, $364 \mathrm{p}$.

\section{BOSINSKI G., FISCHER G.}

1974: Die Menschendarstellungen von Gönnersdorf der Ausgrabung von 1968, Wiesbaden, Franz Steiner Verlag, 131 p.

\section{BREUIL H.}

1952 : Quatre cents siècles d'art pariétal : les cavernes ornées de l'âge du Renne, Montignac, Centre d'études et de documentations préhistoriques, $413 \mathrm{p}$.

BROGLIO A., DALMERI G. (DIR.)

2003 : Pitture paleolitiche nelle Prealpi Venete: grotta di Fumane e riparo Dalmeri, Verona, Cierre ed. (coll. Memoire del museo civico di Storia naturale di Verona, 2, n spécial Prehistoria alpina), 191 p.

\section{Clottes J.}

1999 : La Vie et l'art des Magdaléniens en Ariège -2- Voyage en préhistoire, Paris, Maison des torique), $700 \mathrm{p}$ Roches (coll. Histoire de la France préhis-

\section{CréMAdès M.}

1994 : " L'art mobilier paléolithique : analyse des procédés technologiques ", Complutum, 5, p. 369-384.

Dalmeri G., BassetTi M., Cusinato A., KOMPATSCHER K., HROZNY-

KOMPATSCHER M., NICOLODI F.

2003a : " L'insieme dell'arte mobiliare. Le pitture 2002 : primi dati ", in BROGLIO A., DALMERI G. (DIR.), Pitture paleolitiche nelle Prealpi Venete : grotta di Fumane e riparo Dalmeri, Verona, Cierre ed. (coll. Memoire del museo civico di Storia naturale di Verona, 2, $\mathrm{n}^{\circ}$ spécial Preistoria alpina), p. $33-144$

Dalmeri G., BassetTi M., Cusinato A., KOMPATSCHER K., HROZNY-

KOMPATSCHER M., LANZINGER M.

2003b : Le Pietre dipinte del sito epigravettiano di riparo Dalmeri : campagna di ricerche 2001, Trento, Museo Tridentino di Scienze naturali, p. 3-34.

Delluc B., Delluc G.

1983 : « Les grottes ornées de Domme (Dordogne) : la Martine, le Mammouth et le Pigeonnier ", Gallia Préhistoire, 26, p. 7-80.

1984 : " Lecture analytique des supports rocheux gravés et relevé synthétique », L'Anthropologie, 88, p. 511-519.

\section{D'ERRICO F}

1988 : « Lecture technologique de l'art mobilier gravé : nouvelles méthodes et premiers résultats sur les galets gravés de Rochedane ", L'Anthropologie, 92, p. 101-122.

1993 : "Identification des traces de manipulation, suspension, polissage sur l'art mobilier en os, bois de cervidés, ivoire ", in Traces et fonction : les gestes retrouvés, Actes du colloque international de Liège, 8-10 déc. 1990, Liège (coll. ERAUL, 50, vol. 1), p. 177-188.

1994 : L'Art gravé azilien : de la technique à la signification, Paris, CNRS Éditions (coll. Suppl. à Gallia Préhistoire, XXXI), 329 p.

D'ERRICO F., ESPINET-MOUCADEL J.

1986 : «L'emploi du microscope électronique à balayage pour l'étude expérimentale de traces d'usure : raclage sur bois de cervidé ", Bulletin de la Société préhistorique française, 83, 3, p. 91-96. 
FISCHER G.

1979 : «Eine Jagdszene aus Gönnersdorf », Archäologisches Korrespondenzblatt, 9, p. 243-249

\section{Fritz C.}

1999 : La Gravure dans l'art mobilier magda lénien, Paris, Maison des sciences de l'homme, $216 \mathrm{p}$.

\section{Fritz C., TOSEllo G., WAlter P.}

1993 : «La gravure sur os au Magdalénien : étude microscopique d'une côte de la grotte de La Vache (commune d'Alliat, Ariège) ", Bulletin de la Société préhistorique française, 90, p. 411-425.

LEJEUNE M., Welté A.-C. (DIR.)

2004 : L’Art du Paléolithique supérieur, Actes $d u X I V^{e}$ congrès de l'Union internationale des sciences préhistoriques et protohistoriques, Université de Liège, 2-8 sept. 2001, Liège (coll. ERAUL, 107), 277 p.

\section{LORBLANCHET M.}

1995 : Les Grottes ornées de la Préhistoire, Paris, Errance, 288 p.

\section{LWOFF S.}

1940 : «La Marche, commune de Lussacles-Châteaux (Vienne) : premier atelier du Magdalénien III à dalles gravées mobiles ", Bulletin de la Société préhistorique française, 37, p. 155-180.

1941 : « La Marche, commune de Lussac-lesChâteaux (Vienne) : gravures à représentations d'humains du Magdalénien III, fouilles Péricard L. et Lwoff S. », Bulletin de la Société préhistorique française, 38, 8, p. 145-161.

1943 : «La Marche : iconographie humaine du Magdalénien III », Bulletin de la Société préhistorique française, 40, 7-9, p. 166-180.

1957 : «Grotte de la Marche : iconographie humaine et animale du Magdalénien III », Bulletin de la Société préhistorique française, 54,10 , p. 622-633.

1959a [paru un 1965] : Deux conséquences de la découverte de la Marche, Actes du congrès préhistorique de Monaco, $16^{e}$ session, Ajaccio, p. 838-844.

1959b : «La Marche : lampes et ménisques, burins atypiques ", Bulletin de la Société préhistorique française, 56, 5-6, p. 327-335.

1962a : «Grotte de la Marche : industrie de l'os, iconographie humaine et animale », Bulletin de la Société préhistorique française, 59, 1-2, p. 73-91.

$1962 \mathrm{~b}:$ : Industrie microlithique du Magdalénien III et industrie lithique du Magdalénien IV-V : grotte de La Marche, commune de Lussac-les-Châteaux (Vienne) », Bulletin de la Société préhistorique française, 59, 7-8, p. 499-510.
1965 : «Grotte de la Marche (Lussac-lesChâteaux, Vienne). Industrie lithique : perçoirs du Magdalénien III ", Bulletin de la Société préhistorique française, 61, p. 271-288.

\section{MARSHACK A.}

1970 : Notation dans les gravures du Paléolithique supérieur : nouvelles méthodes d'analyse, Bordeaux, Imprimerie Delmas, 124 p.

\section{MÉLARD N.}

2006a : «Lire l'invisible : la microtopographie ", Archéologia, 429, p. 12-13.

2006b : Les Pierres gravées du Magdalénien moyen de La Marche (Lussac-les-Châteaux, Vienne) réalisation, fonctions et interprétations, thèse de Doctorat, Paris, Muséum national d'histoire naturelle, 2 vol., 389 p.

\section{MOHEN J.-P.}

2002 : Arts et Préhistoire : naissance mythique de l'humanité, Paris, Terrail, 207 p.

\section{PAILleT P.}

1999 : Le Bison dans les arts magdaléniens du Périgord, Paris, CNRS Éditions (coll. Suppl à Gallia Préhistoire, XXXIII), 475 p.

\section{Pales L.}

1970 : «Le "coco des roseaux" ou la fin d'une erreur ", Bulletin de la Société préhistorique française, 67,3 , p. $85-88$

Pales L., Tassin de SAINT-Péreuse M.

1964 : «Une scène gravée magdalénienne, grotte de La Marche, Magdalénien III », Objets et Mondes, 4, p. 77-106.

1967 : «Ces dames de La Marche », Objets et Mondes, 7, p. 307-320.

1968 : «Humains superposés de La Marche », in La Préhistoire : problèmes et tendances, Paris, CNRS Éditions, p. 327-336.

1969 : Les Gravures de La Marche -I-Félins et Ours, Bordeaux, Delmas, 272 p., 34 fig., 61 pl. h. t.

1976 : Les Gravures de La Marche -II- Les Humains, Gap, Ophrys, 178 p., 42 fig., 188 pl. h. t.

1981 : Les Gravures de La Marche-III-Équidés et Bovidés, Gap, Ophrys, 150 p., 52 fig., 12 tabl., 124 pl. h. t.

1989 : Les Gravures de La Marche-IV-Cervidés, Mammouths et Divers, Gap, Ophrys, 121 p.

\section{PENVERN I.}

2004 : Le Mammouth laineux (Mammuthus primigenius Blum.) dans le bestiaire paléolithique pariétal en Périgord : étude comparative des grottes de Rouffignac, Font-de-Gaume, Combarelles I et II et Bernifal, thèse de Doctorat, Paris, Muséum national d'histoire naturelle.

\section{PRADEL L.}

1958 : La Grotte magdalénienne de La Marche, commune de Lussac-les-Châteaux (Vienne), Paris, Société préhistorique française (coll. Mémoires de la Société préhistorique française, 5 ).

\section{SAUVET G.}

2004 : « L'art mobilier non classique de la grotte magdalénienne de Bédeilhac (Ariège) ", in LEJEUnE M., Welté A.-C. (DIR.), L'Art du Paléolithique supérieur, Actes $d u X I V^{e}$ congrès de l'Union internationale des sciences préhistoriques et protohistoriques, Université de Liège, 2-8 sept. 2001, Liège (coll. ERAUL, 107), p. 167-176.

\section{SIEVEKING A.}

1987 : «Les plaquettes et leur rôle », in L'Art des objets au Paléolithique-II- Les Voies de la recherche, Actes du colloque international de Foix, 1987, Paris, Direction des Musées de France, p. 7-18.

1988 : «Animaux en action : plaquettes gravées du Magdalénien », L'Anthropologie, 92 , p. $41-50$.

\section{TABORIN Y}

2001 : « De l'art magdalénien à Étiolles (Essonne, Bassin parisien) ", Bulletin de la Société préhistorique française, 98, 1, p. 125-132.

\section{TOSELlO G.}

1985 : Les Pierres gravées inédites du MAN, DEA de Préhistoire, Université Paris-I.

2003 : Pierres gravées du Périgord magdalénien : art, symboles, territoires, Paris, CNRS Éditions (coll. Suppl. à Gallia Préhistoire, XXXVI), 577 p.

Utrilla P., Mazo C., Sopena C., DOMINGO R., NAGORE $O$.

2004 : «L'art mobilier sur pierre du versant sud des Pyrénées : les blocs gravés de la grotte d'Abauntz », in LEJEUnE M., WELTÉ A.-C. (DIR.), L'Art du Paléolithique supérieur, Actes du XIV congrès de l'Union internationale des sciences préhistoriques et protohistoriques, Université de Liège, 2-8 sept. 2001, Liège (coll. ERAUL, 107), p. 199-218.

\section{VIALOU D.}

1976 : Étude descriptive et technologique de l'art gravé de l'Aldène et de l'art gravé-sculpté de la Marche, thèse de Doctorat, Université Paris-I.

1979 : « L'art gravé-sculpté de La Marche », Caesaraugusta, 49-50, p. 23-42.

1982 : «Le relevé d'art préhistorique : une copie d'école ou une analyse ? ", La Recherche, 139, p. 1484-1487.

1984 : «Des blocs sculptés et gravés », Histoire et archéologie, Les Dossiers, 87, p. 70-72. 


\section{PLANCHES}





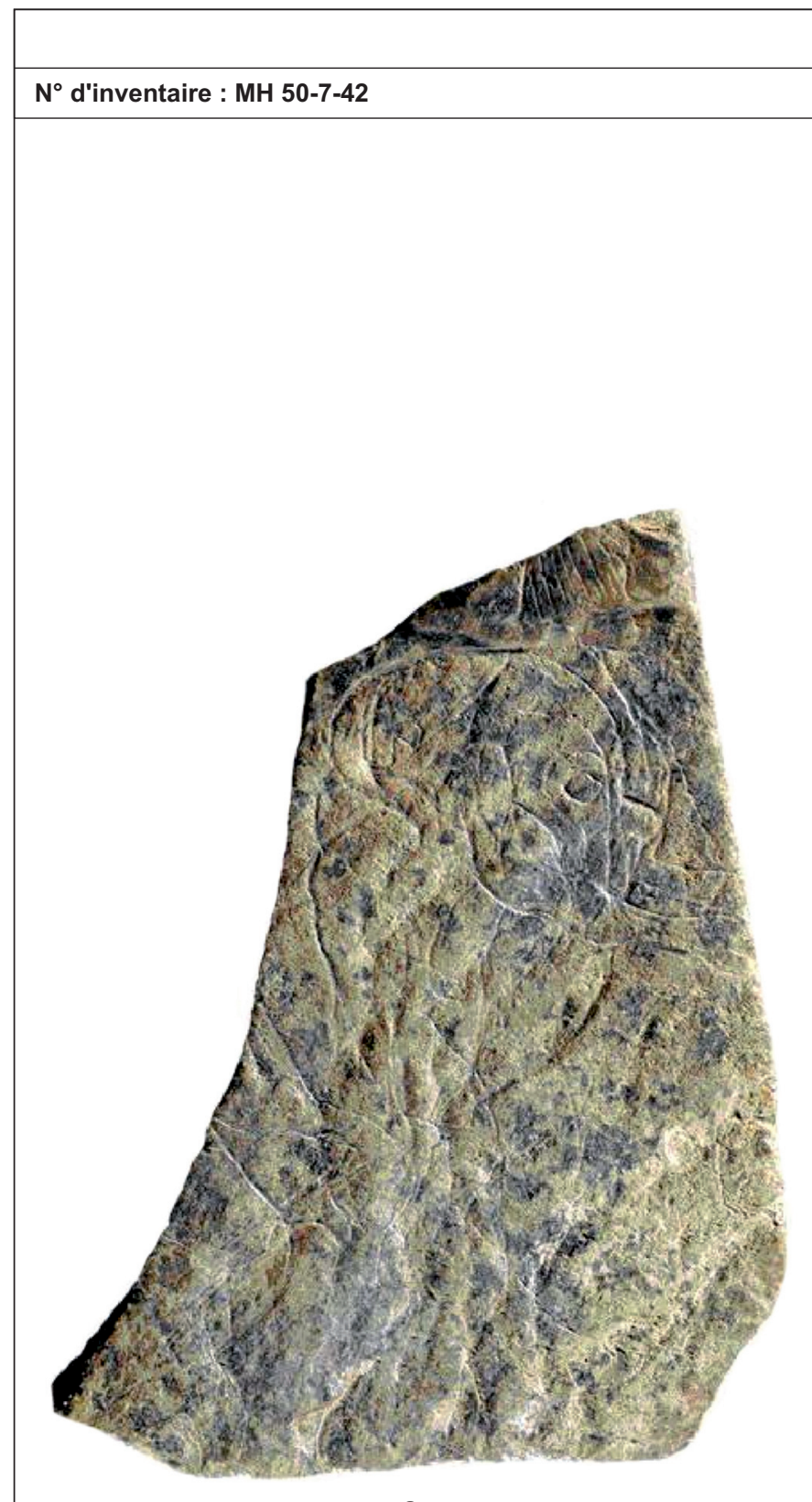

a

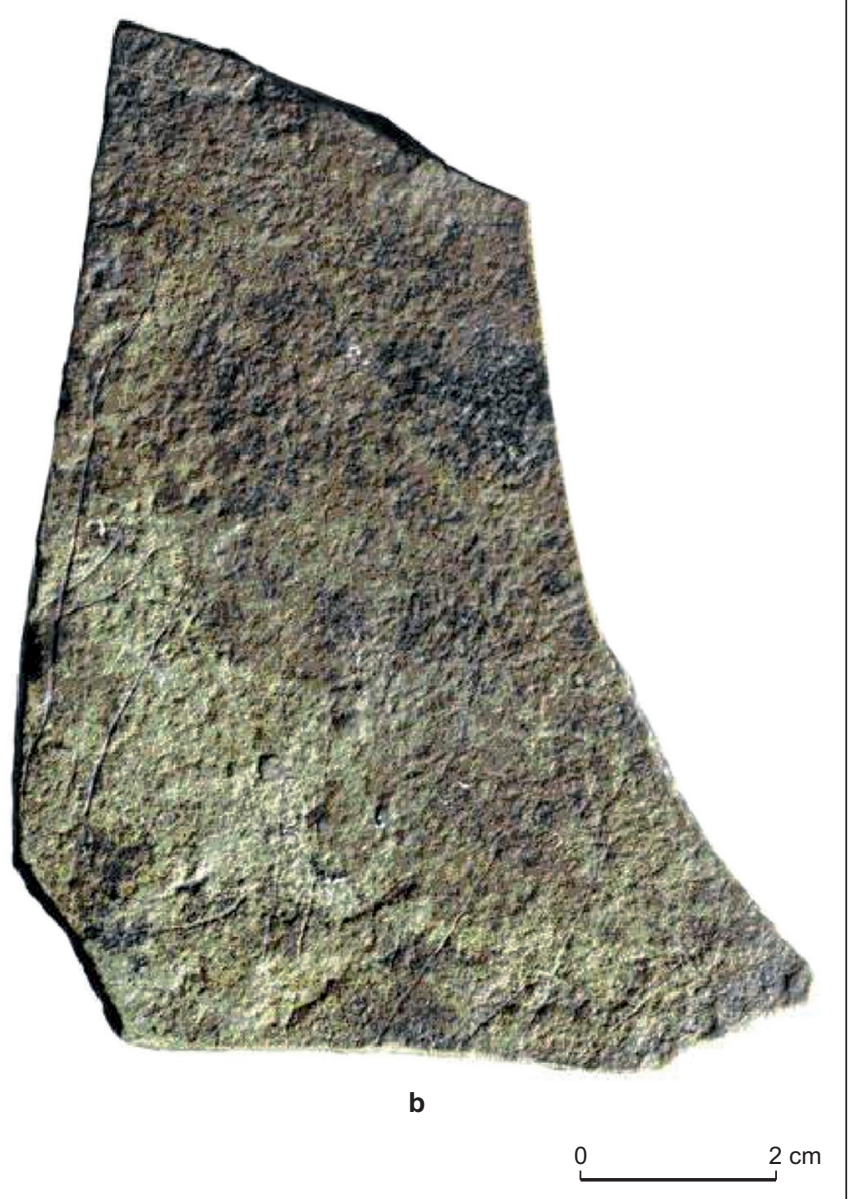

a. photo de la pierre : recto b. photo de la pierre : verso (photos : D. Bagault) 


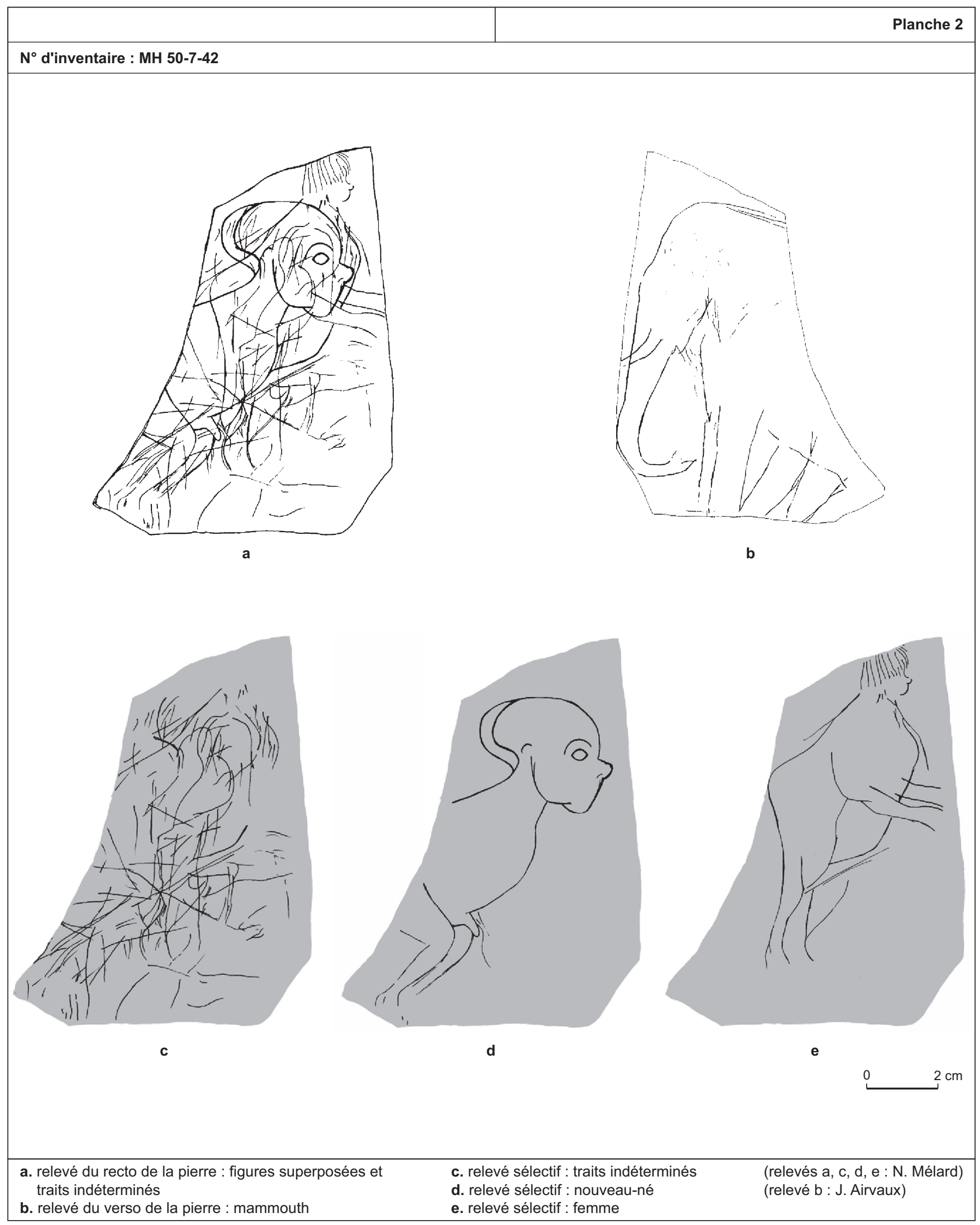




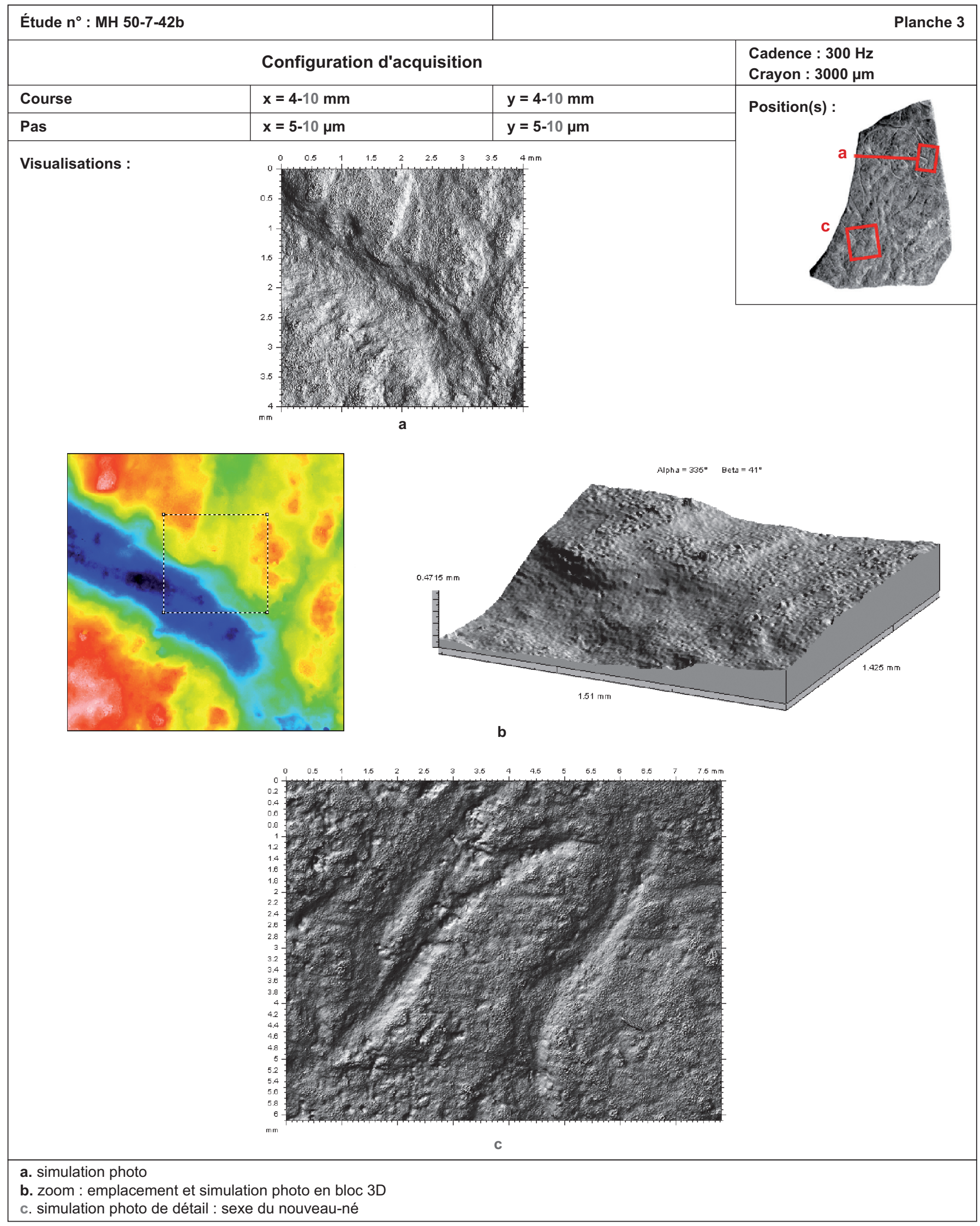




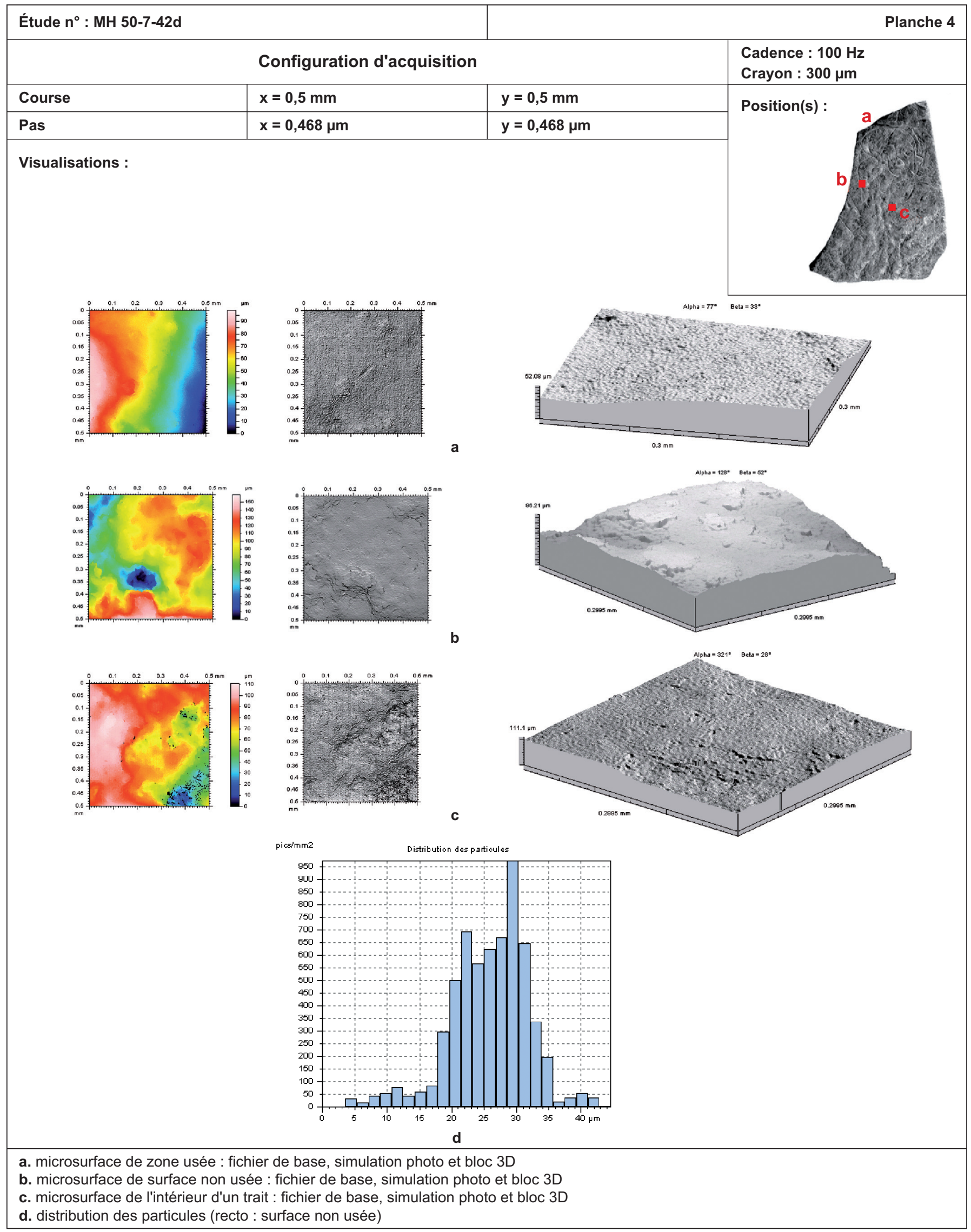




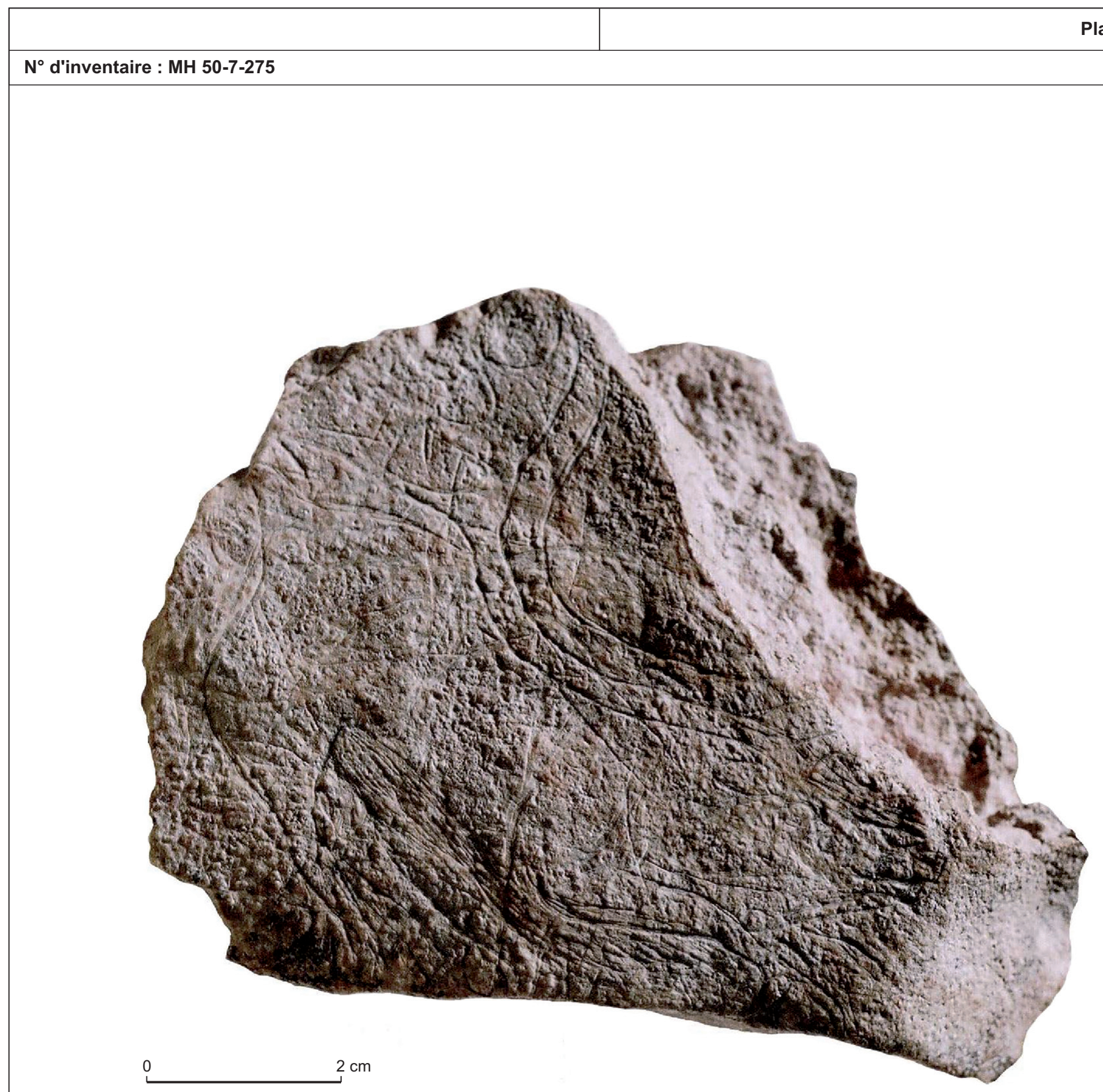




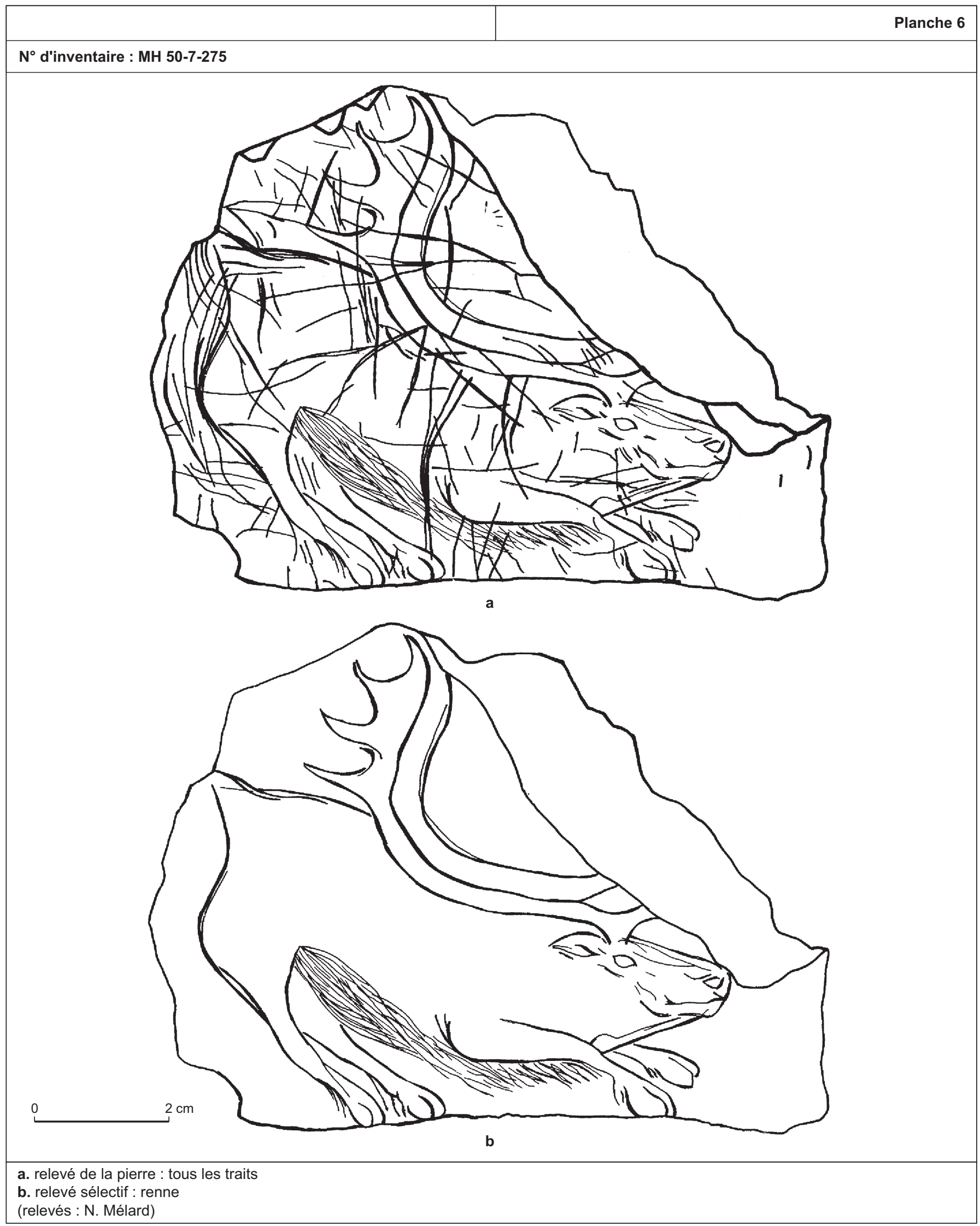




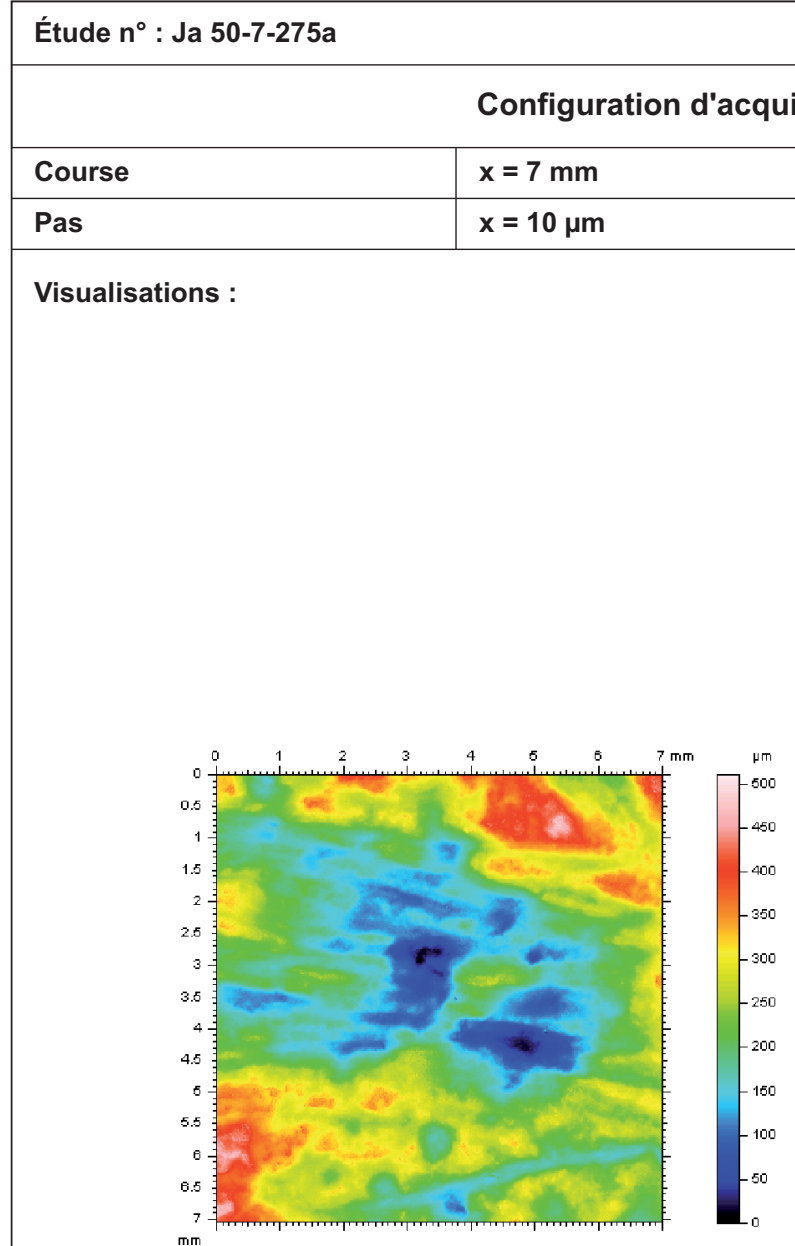

Planche 7

\begin{tabular}{|l|l|l|}
\hline \multicolumn{2}{|l|}{ Étude $\mathrm{n}^{\circ}:$ Ja 50-7-275a } & \multicolumn{2}{l|}{ Configuration d'acquisition } \\
\hline \multicolumn{3}{|l|}{} \\
\hline Course & $x=7 \mathrm{~mm}$ & $y=7 \mathrm{~mm}$ \\
\hline Pas & $x=10 \mu \mathrm{m}$ & $y=10 \mu \mathrm{m}$ \\
\hline
\end{tabular}

Cadence : $100 \mathrm{~Hz}$ Crayon : $\mathbf{3 0 0 0} \mu \mathrm{m}$

Position(s) :

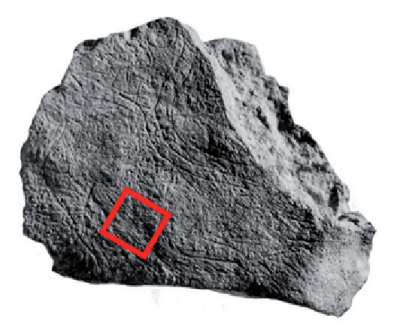

$$
\text { mim }
$$
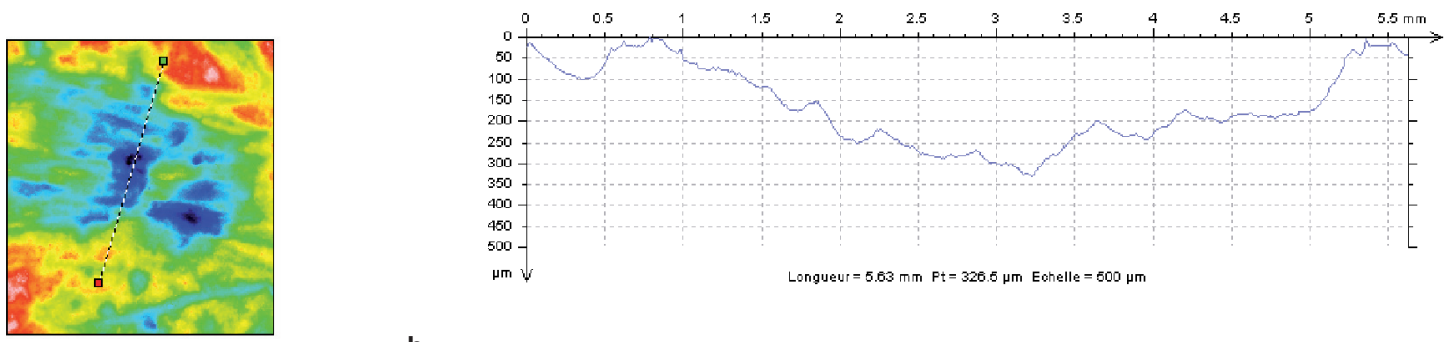

b
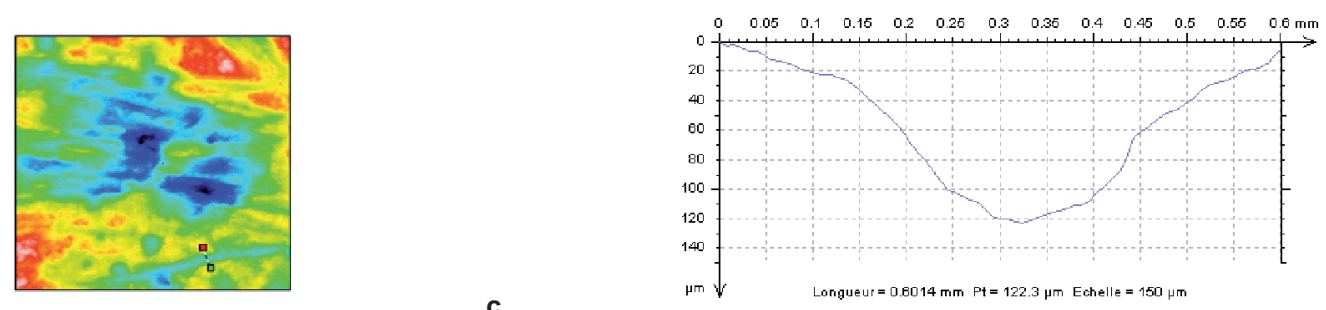

a. fichier de base et simulation photo du détail figuratif : pelage du ventre b. profil 1 : emplacement et profil (pelage)

c. profil 2 : emplacement et profil (trait simple) 


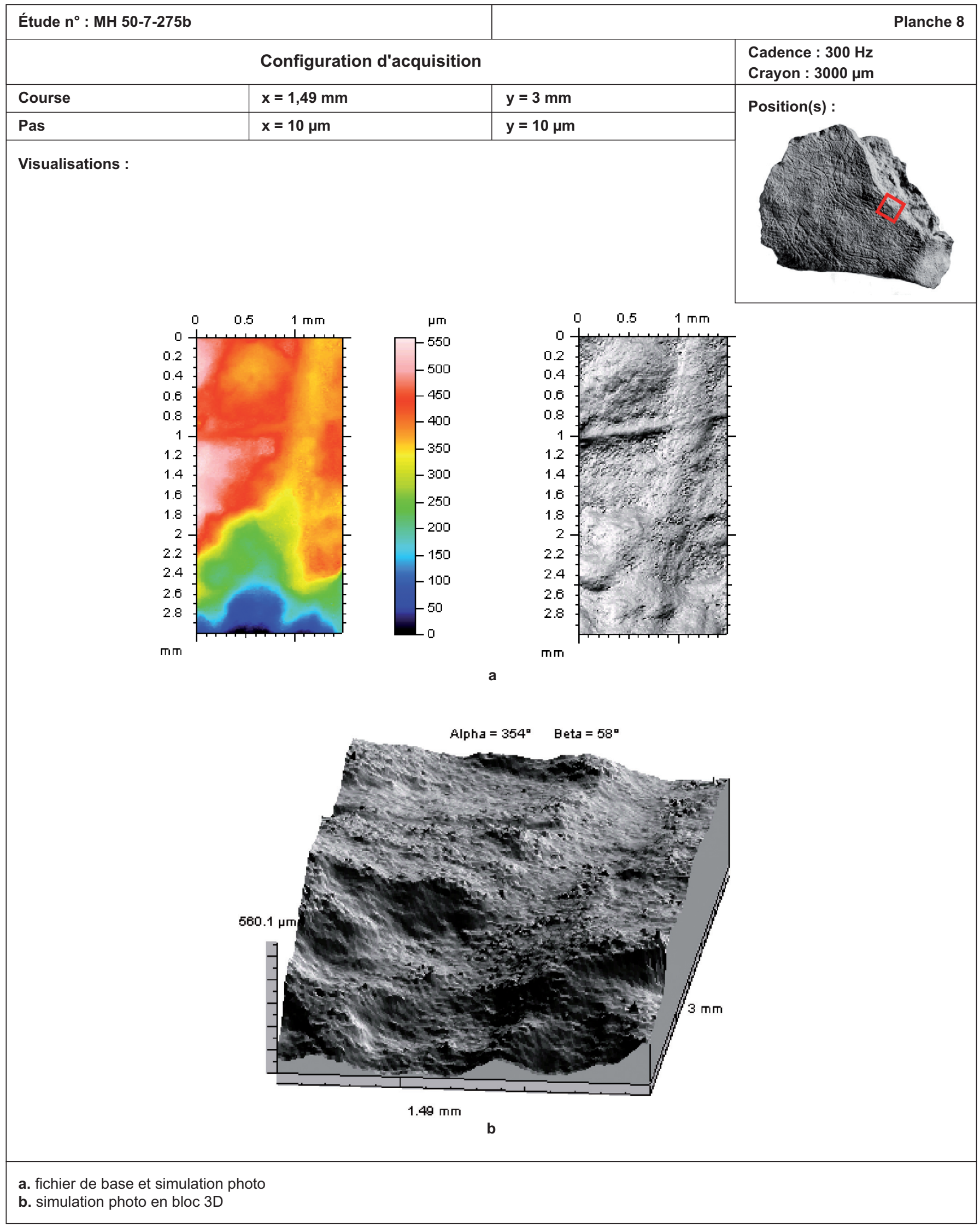




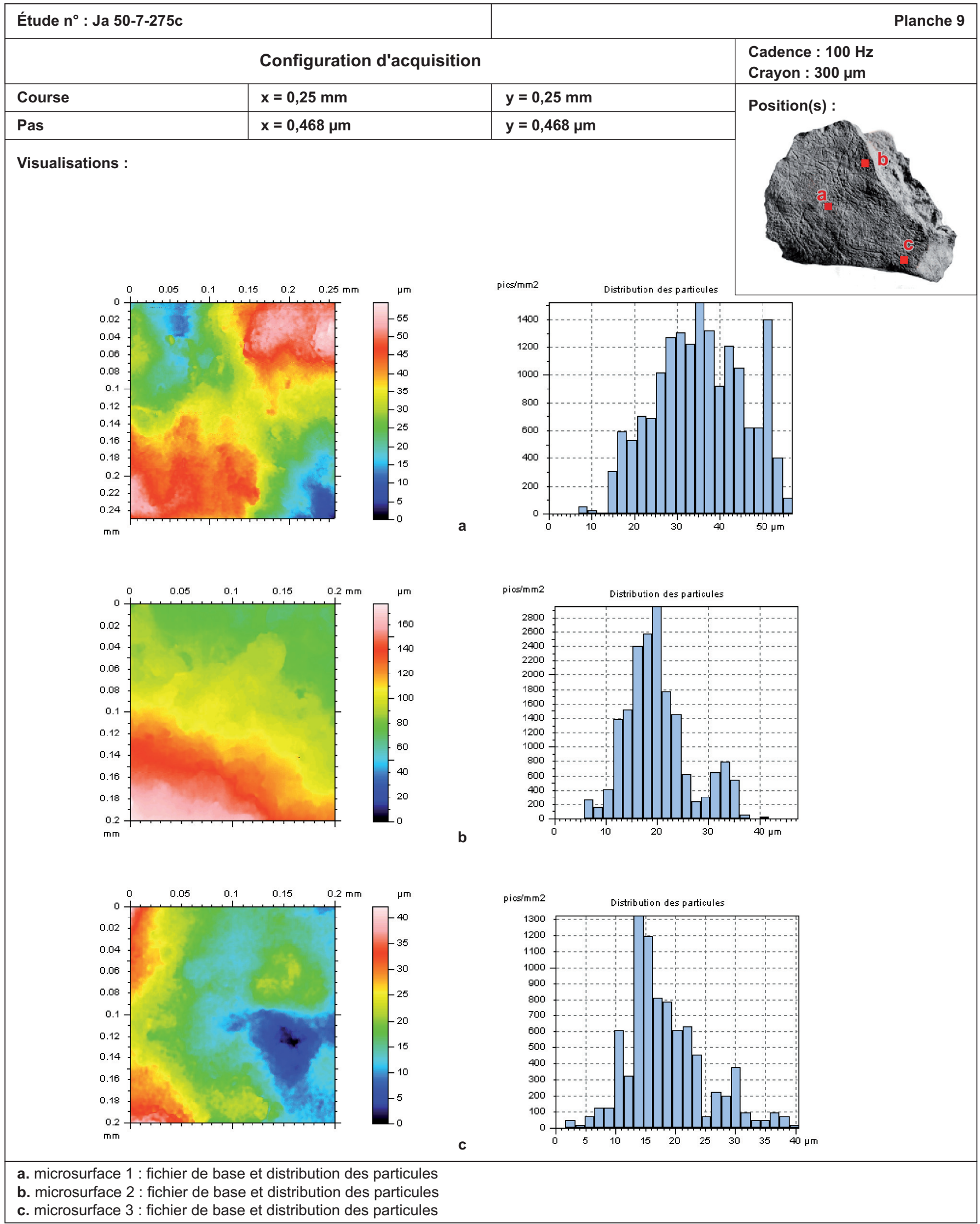


NICOLAS MÉLARD

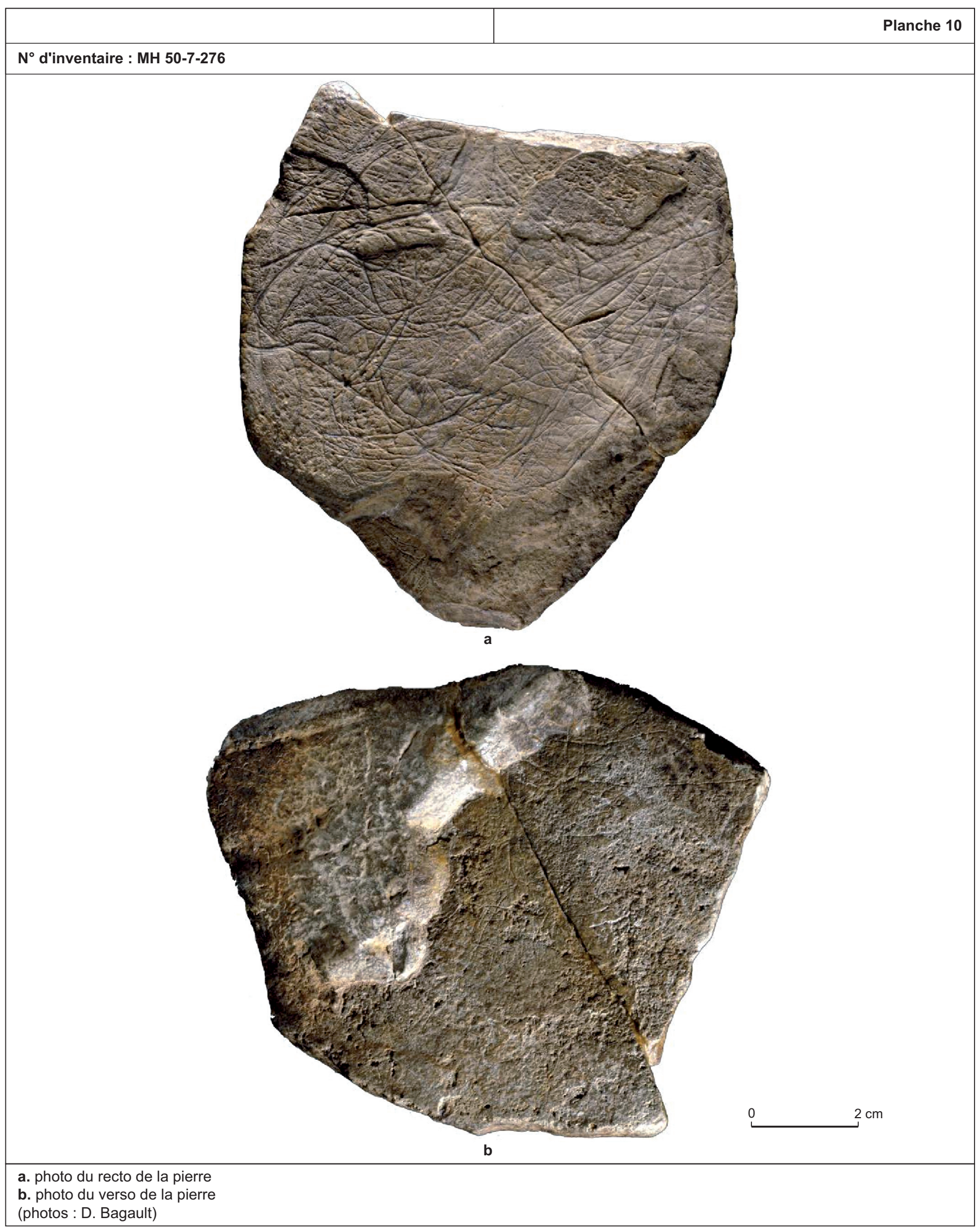




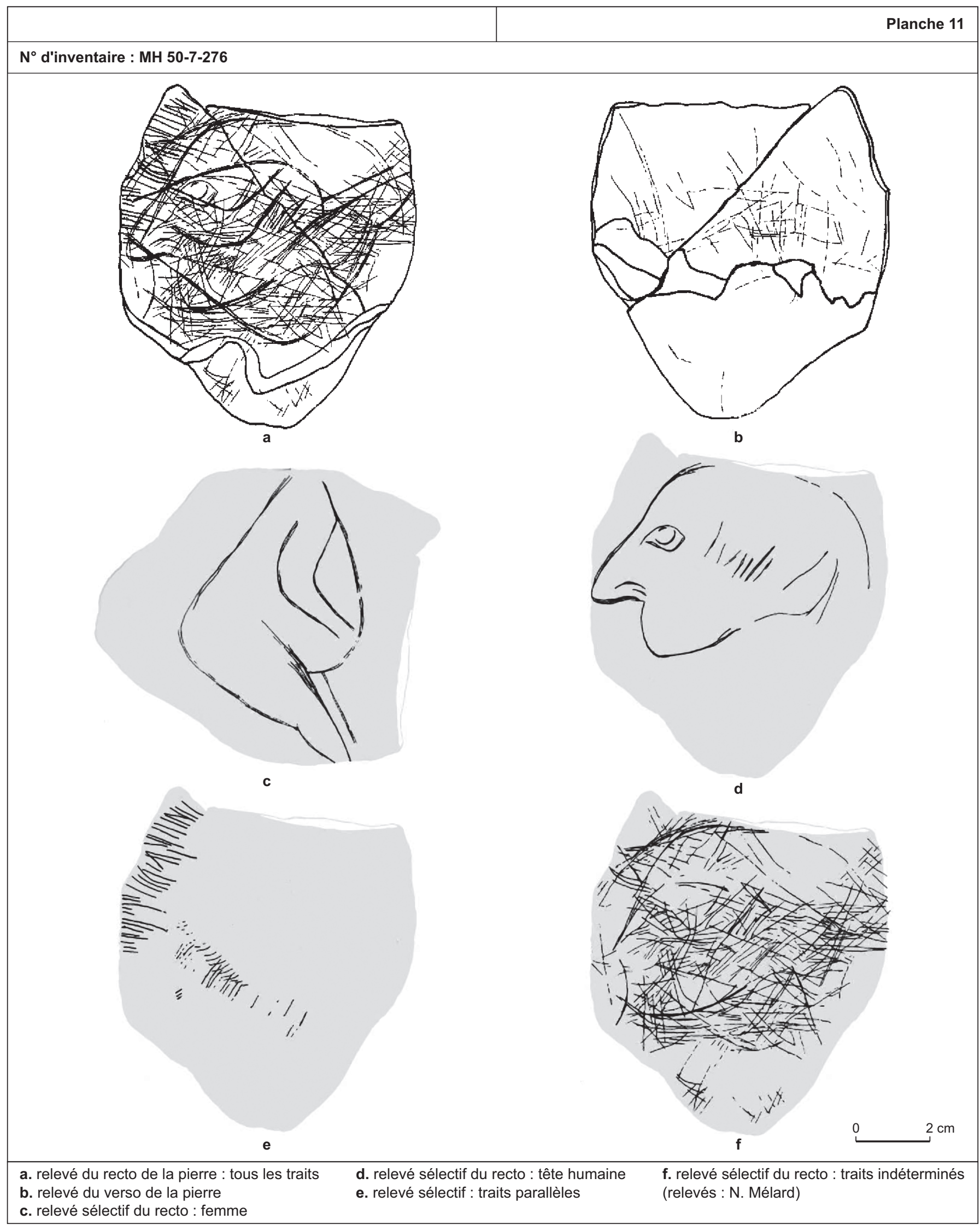




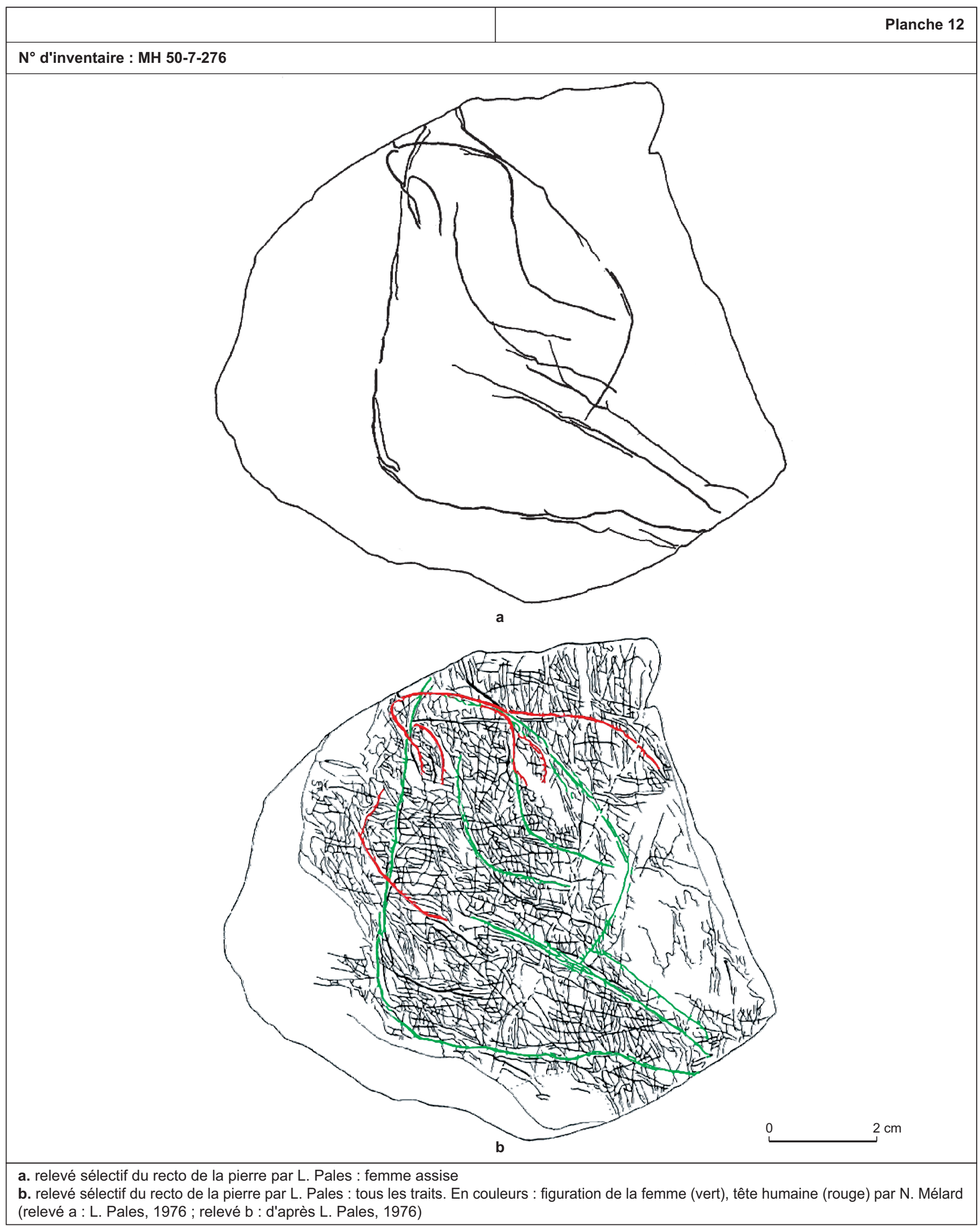




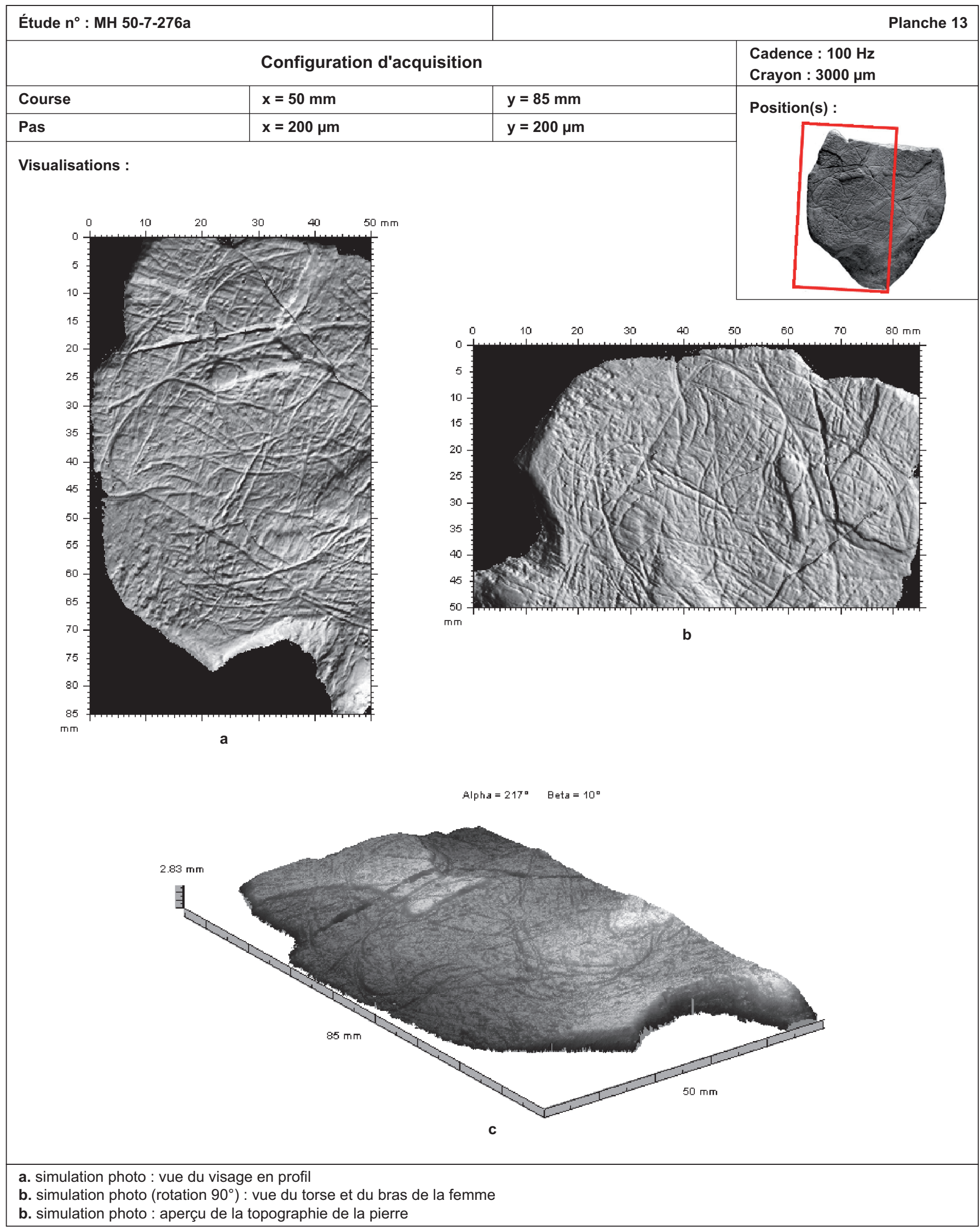




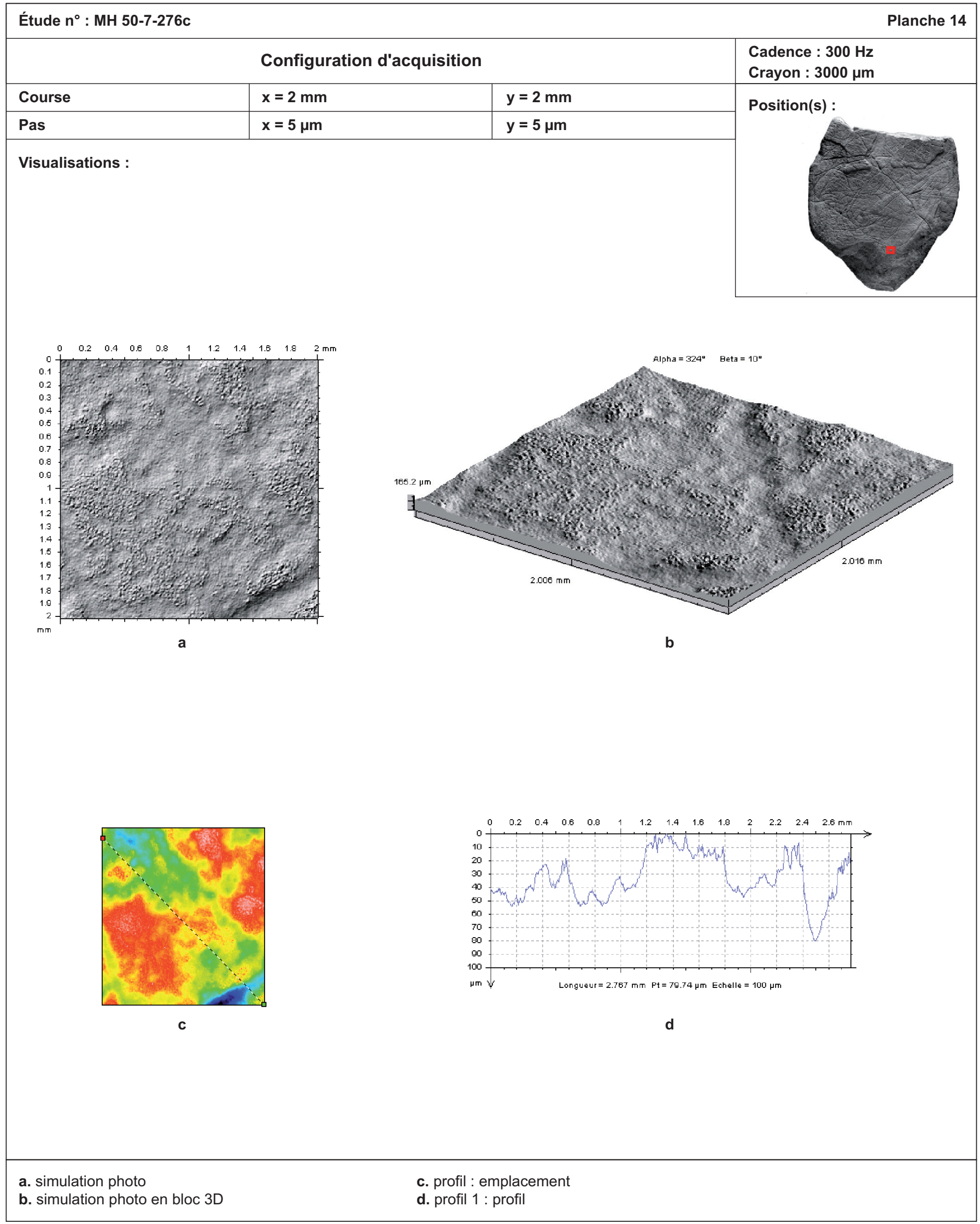




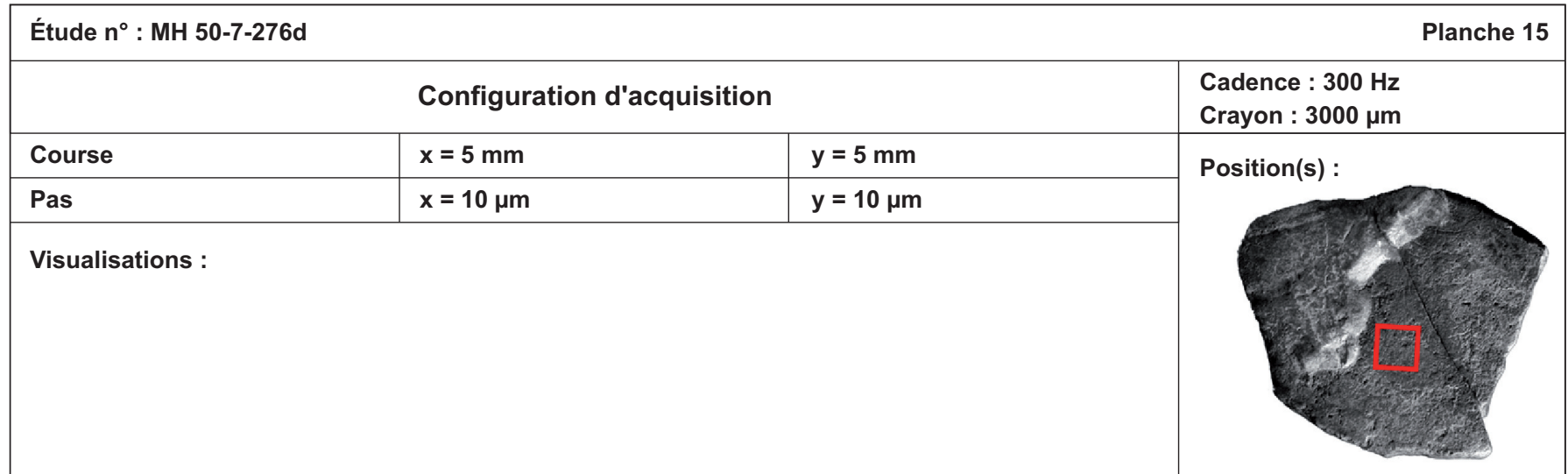
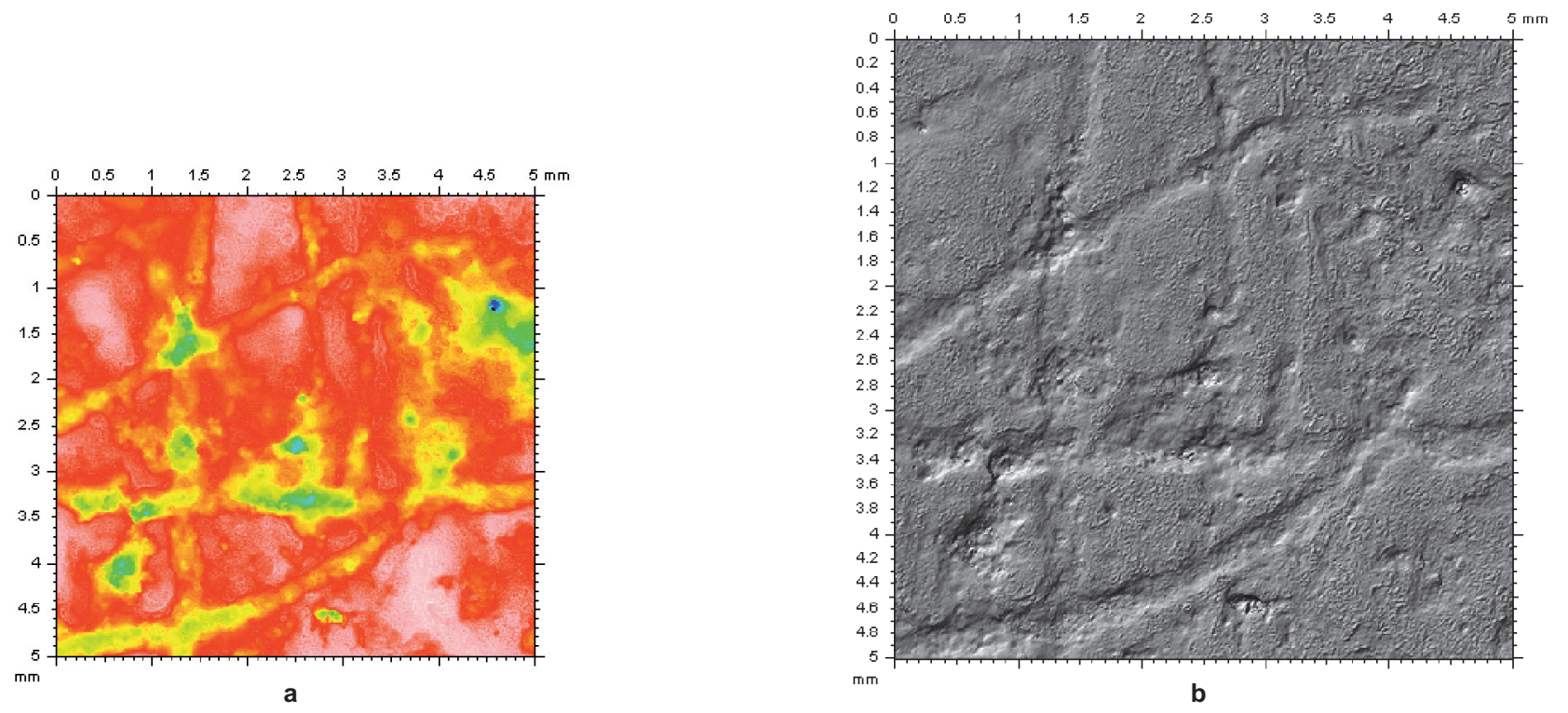

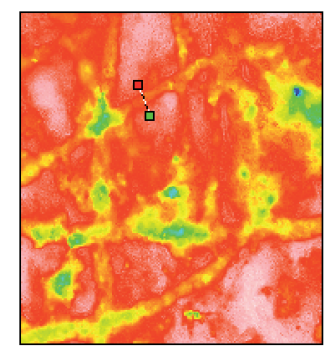

C

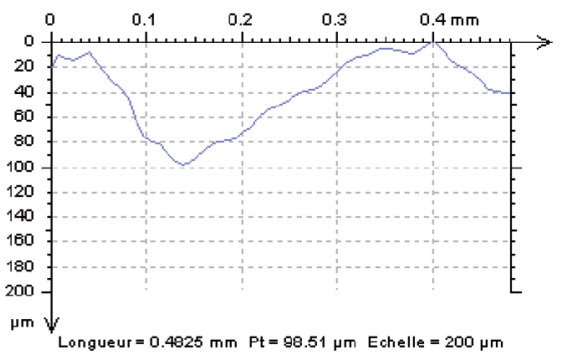

d a. fichier de base

b. simulation photo des traits usés sur le verso c. profil : emplacement

d. profil : profil 


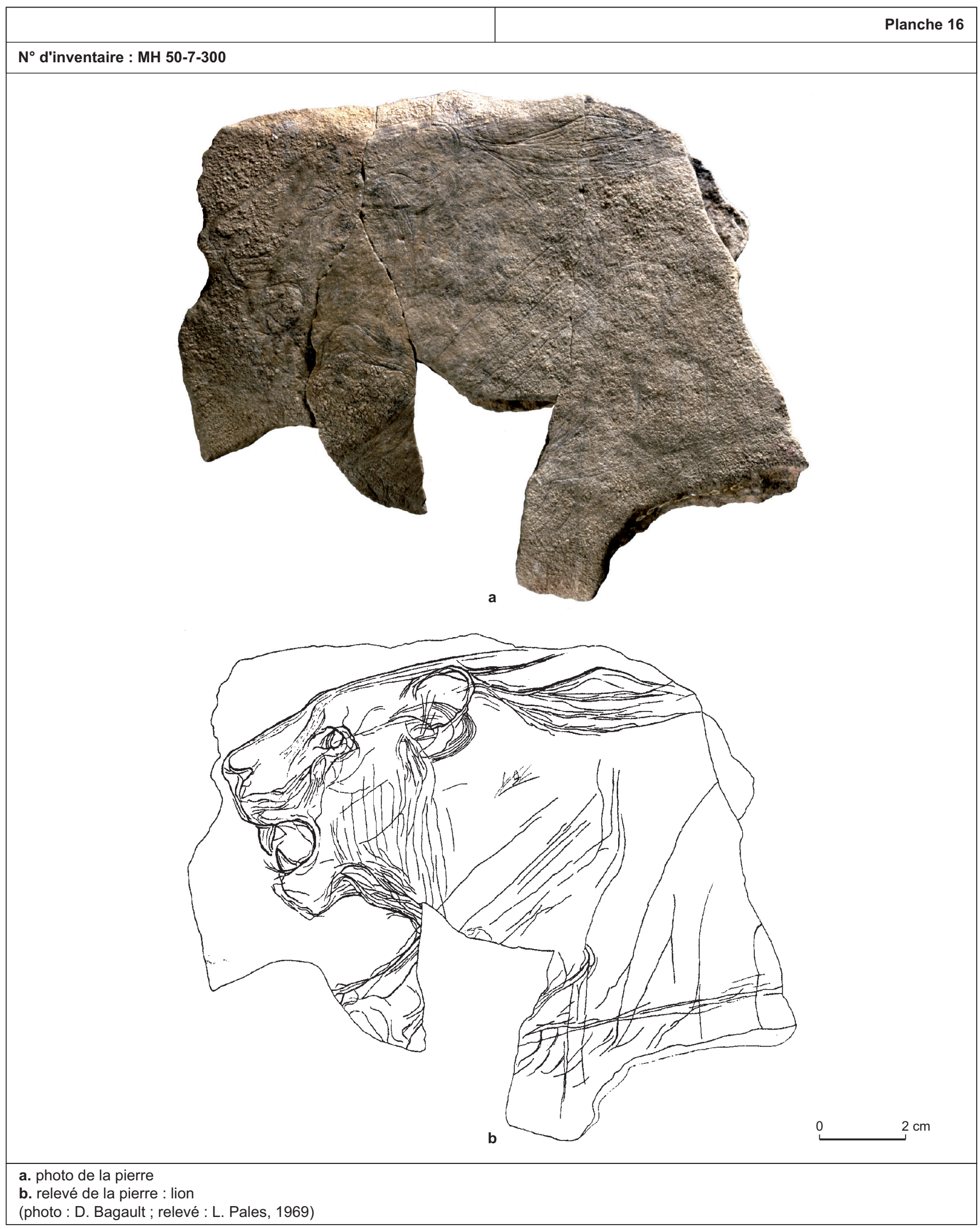




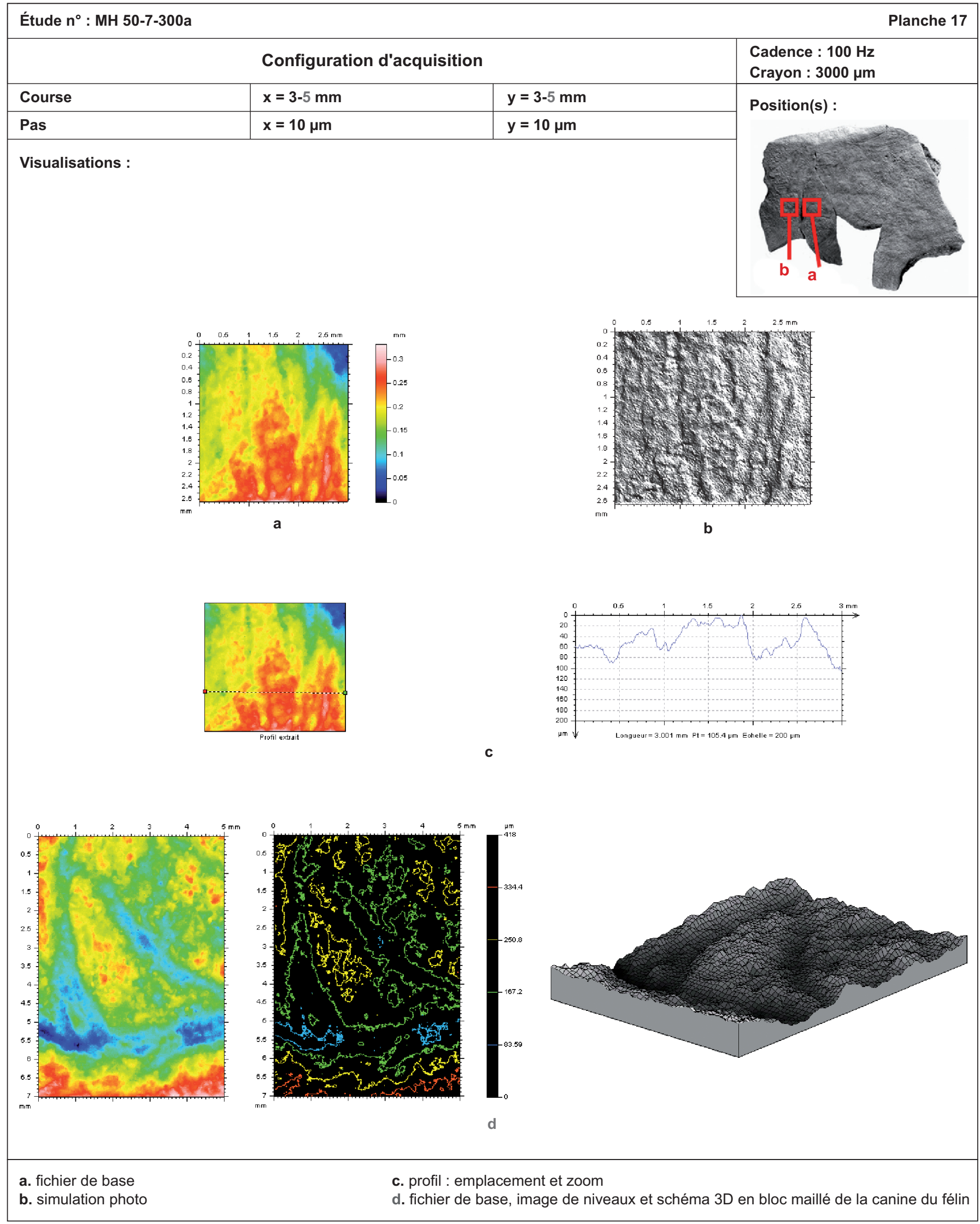




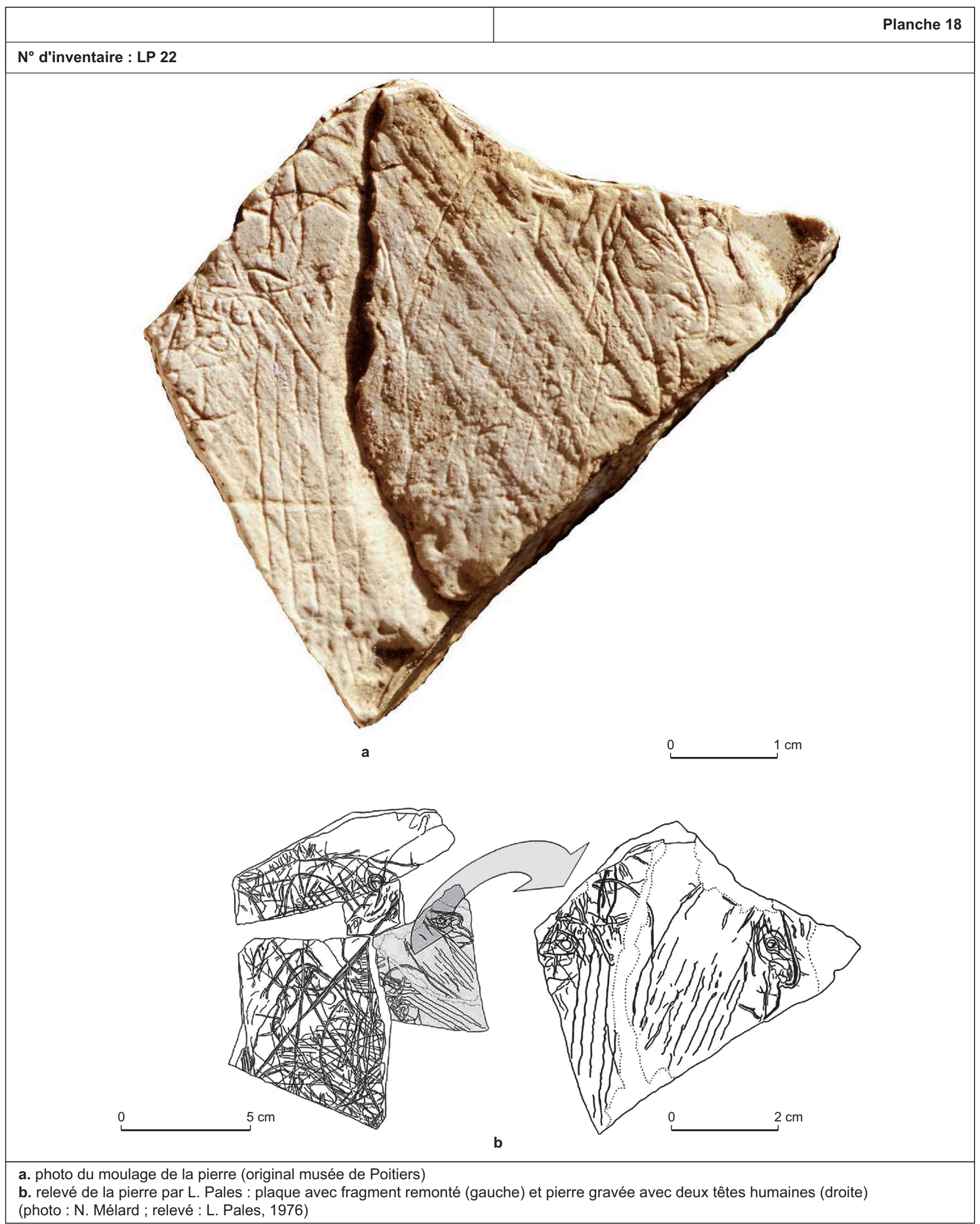




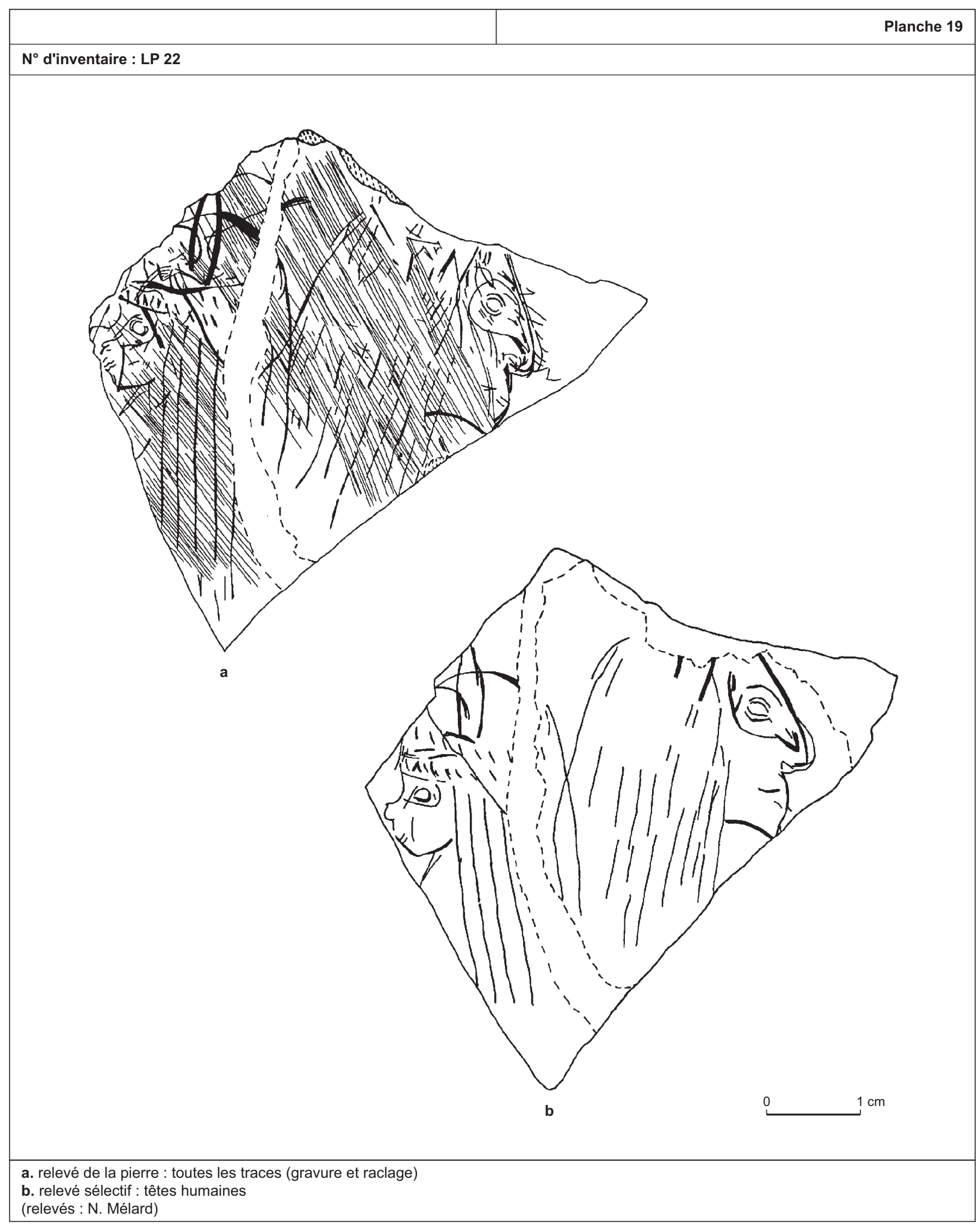




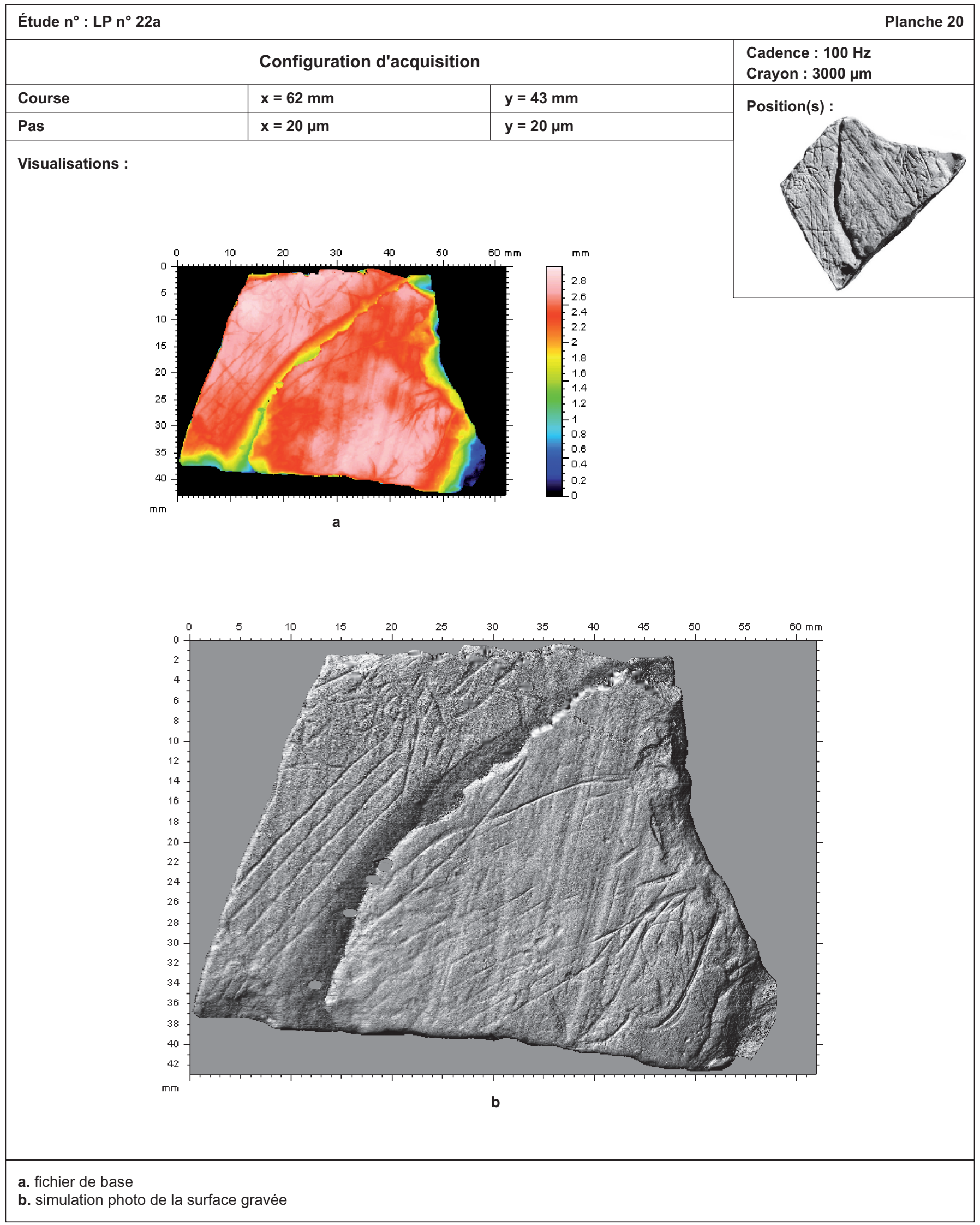




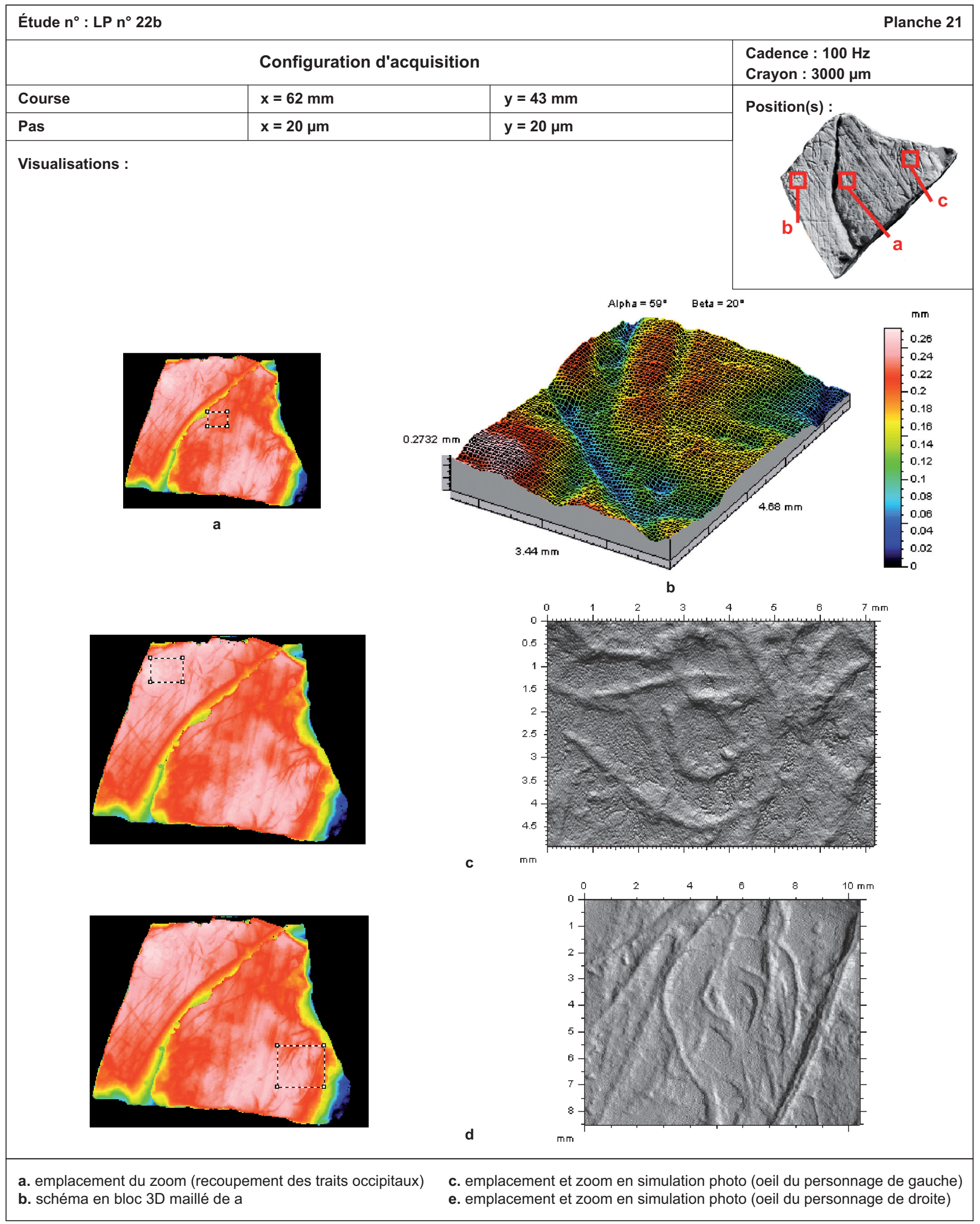


NiCOLAS MÉLARD

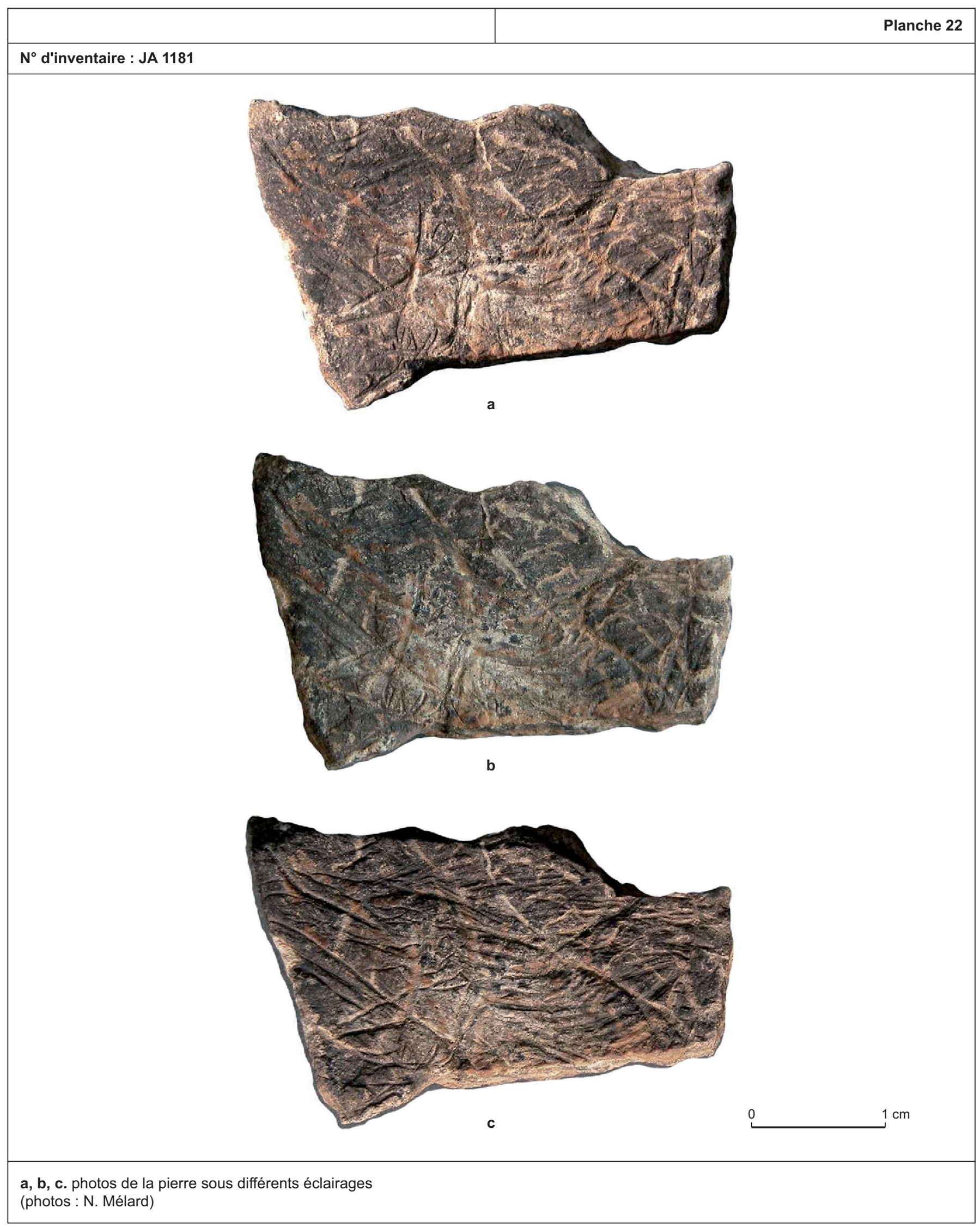




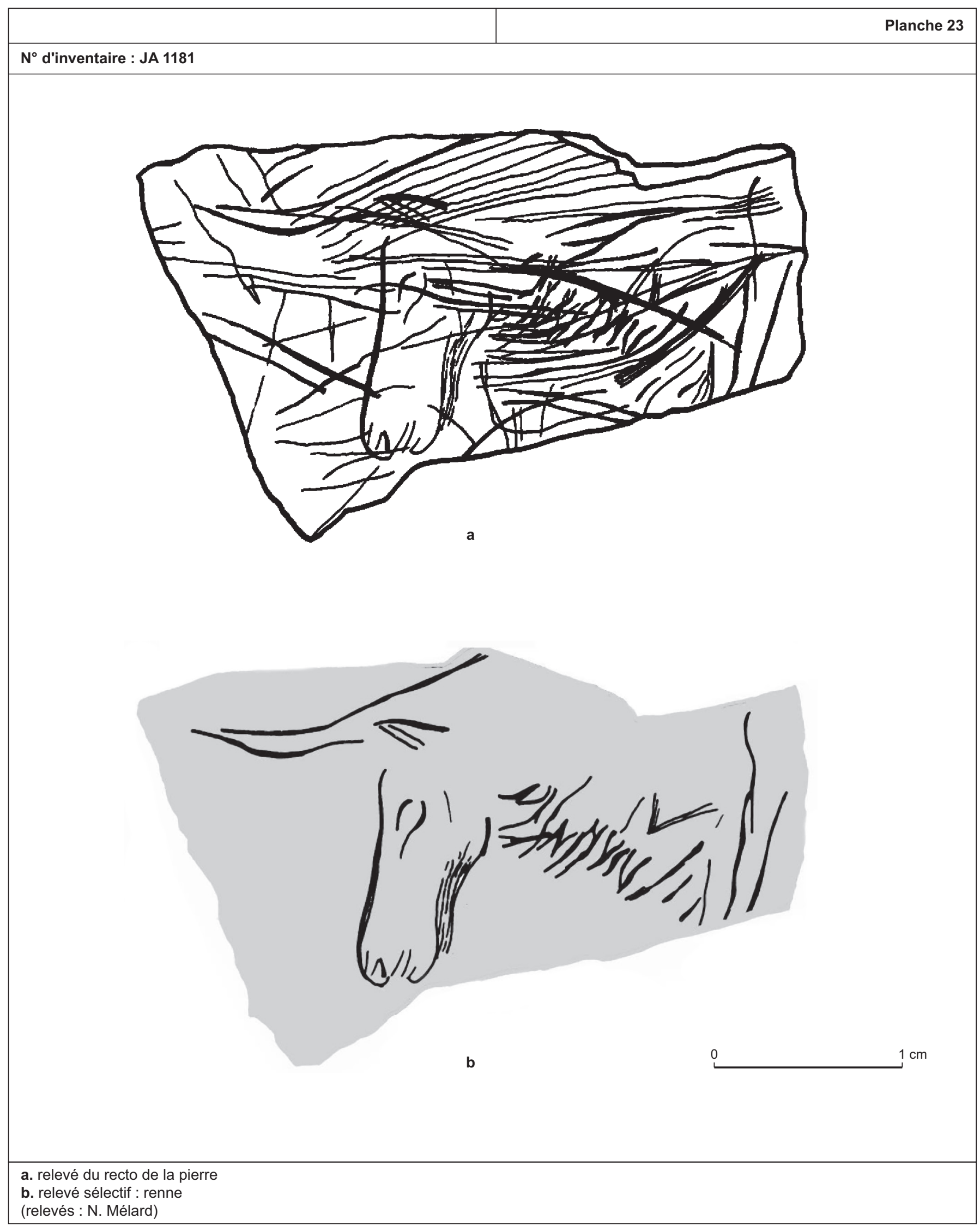

(relevés : N. Mélard) 


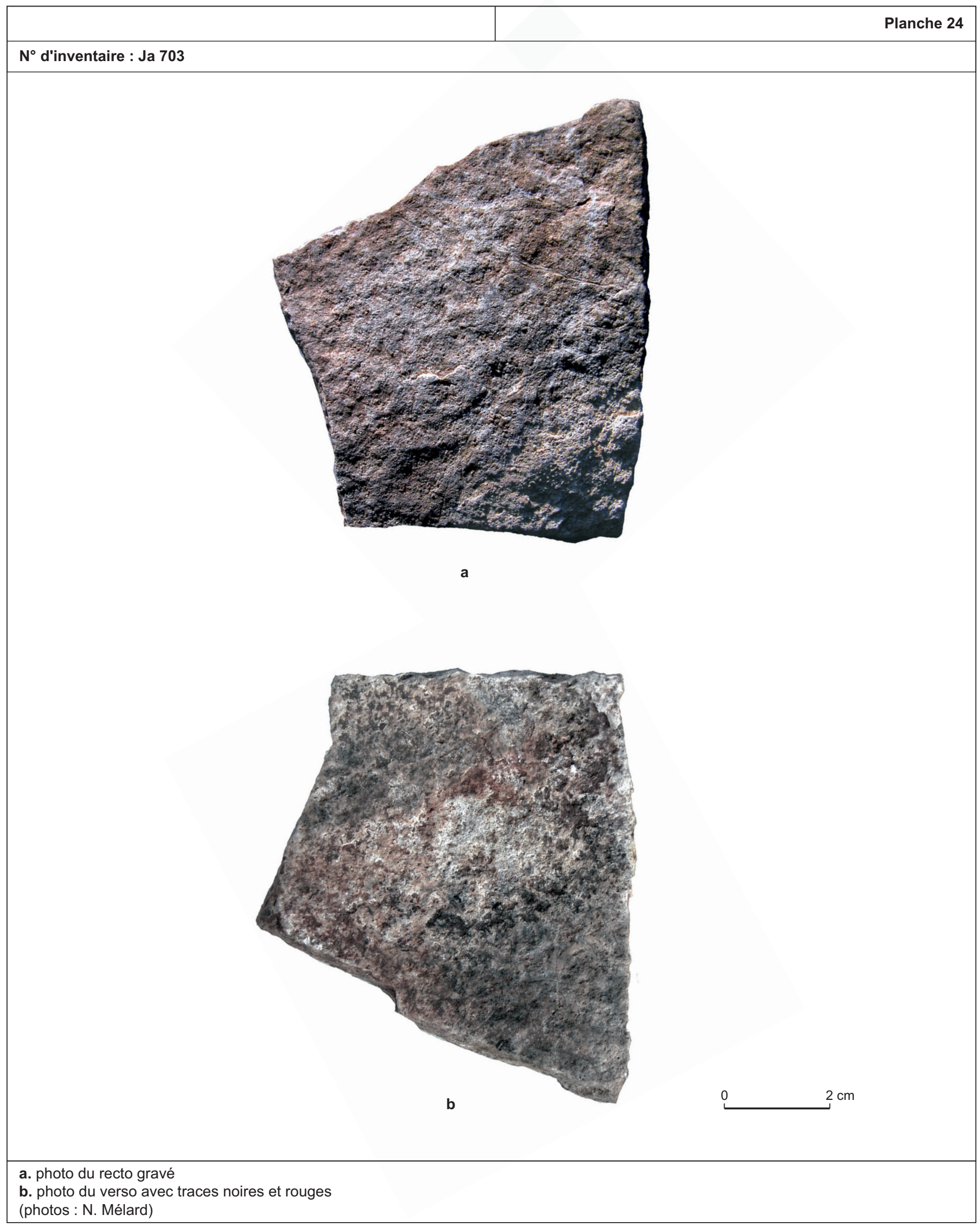




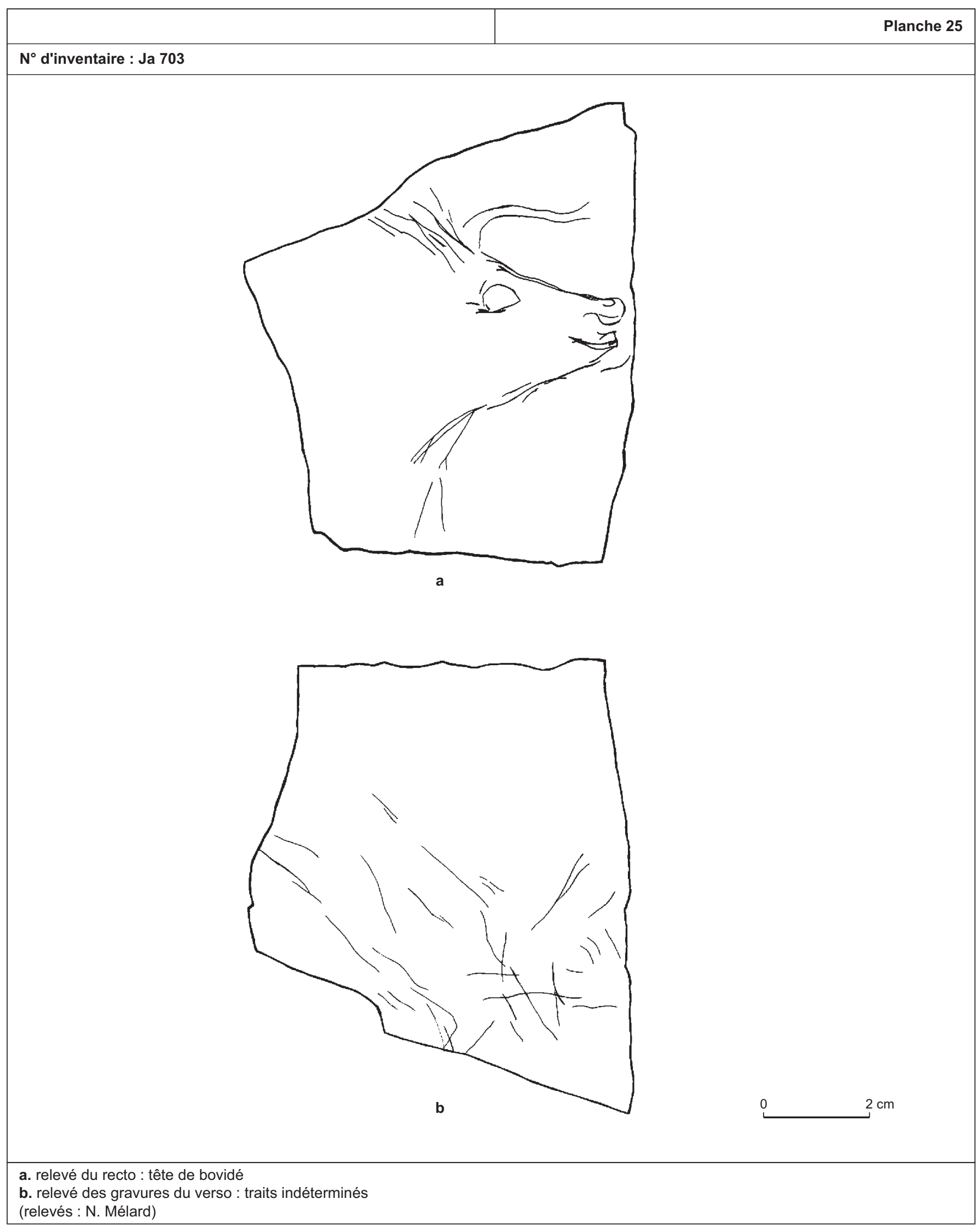




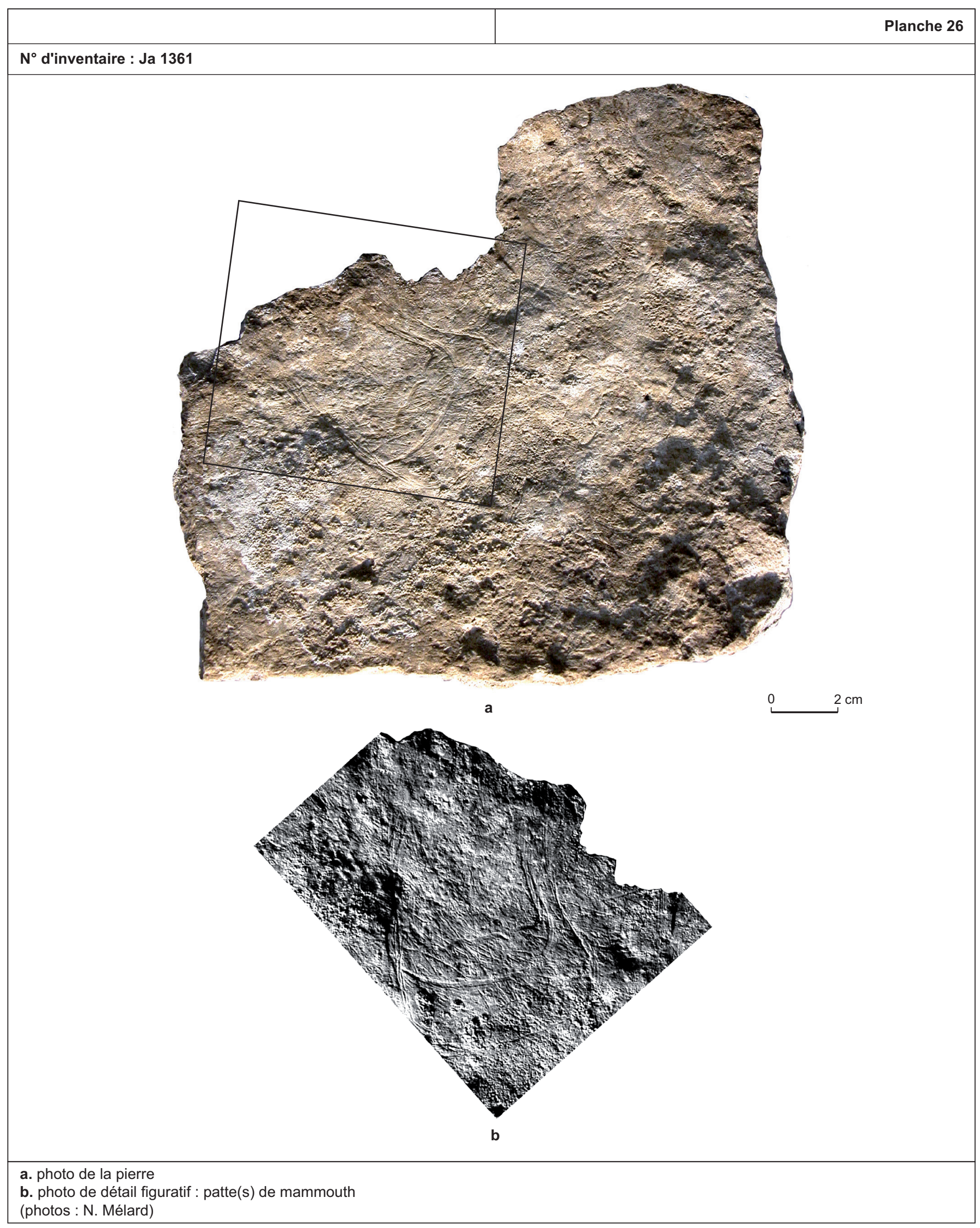




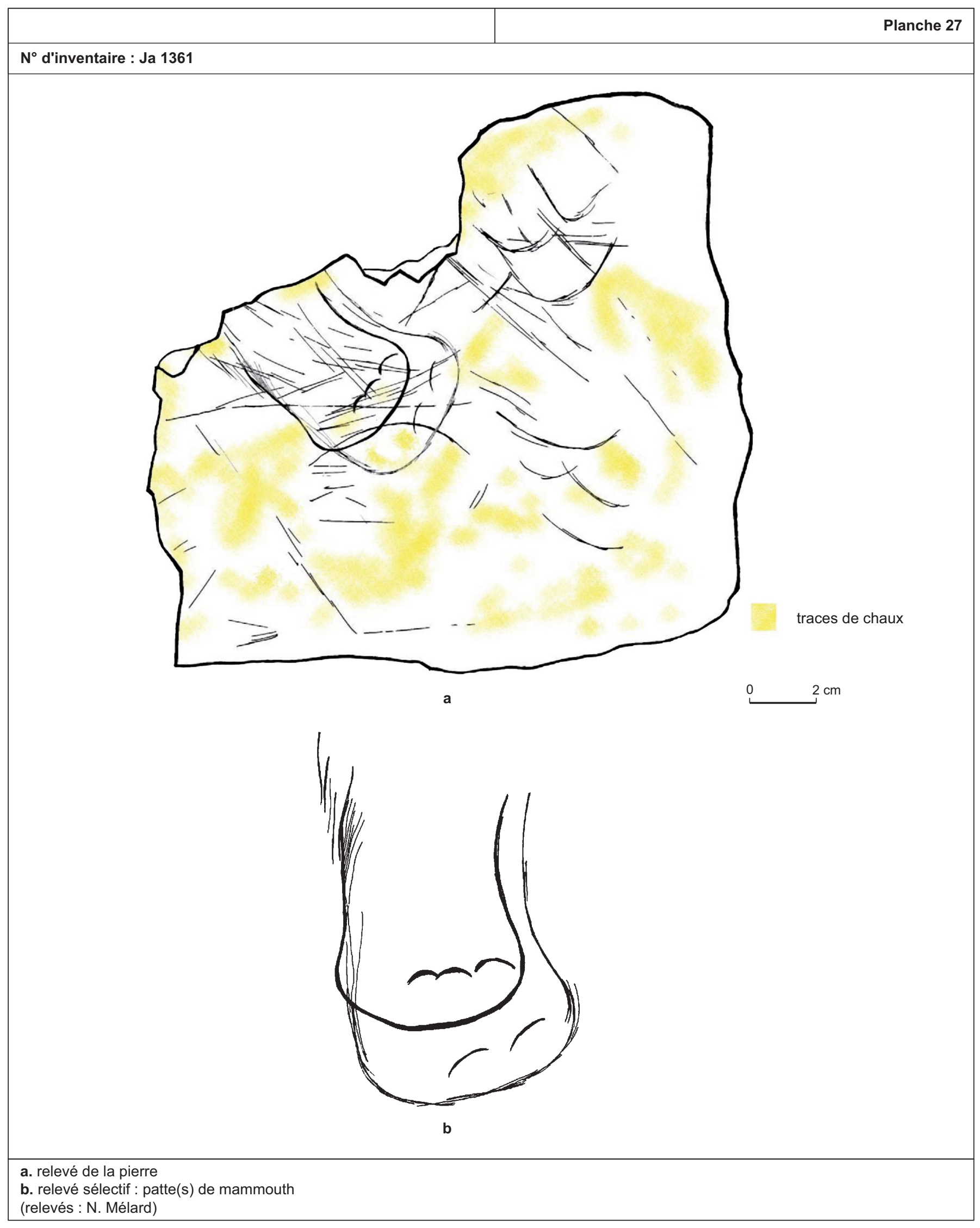




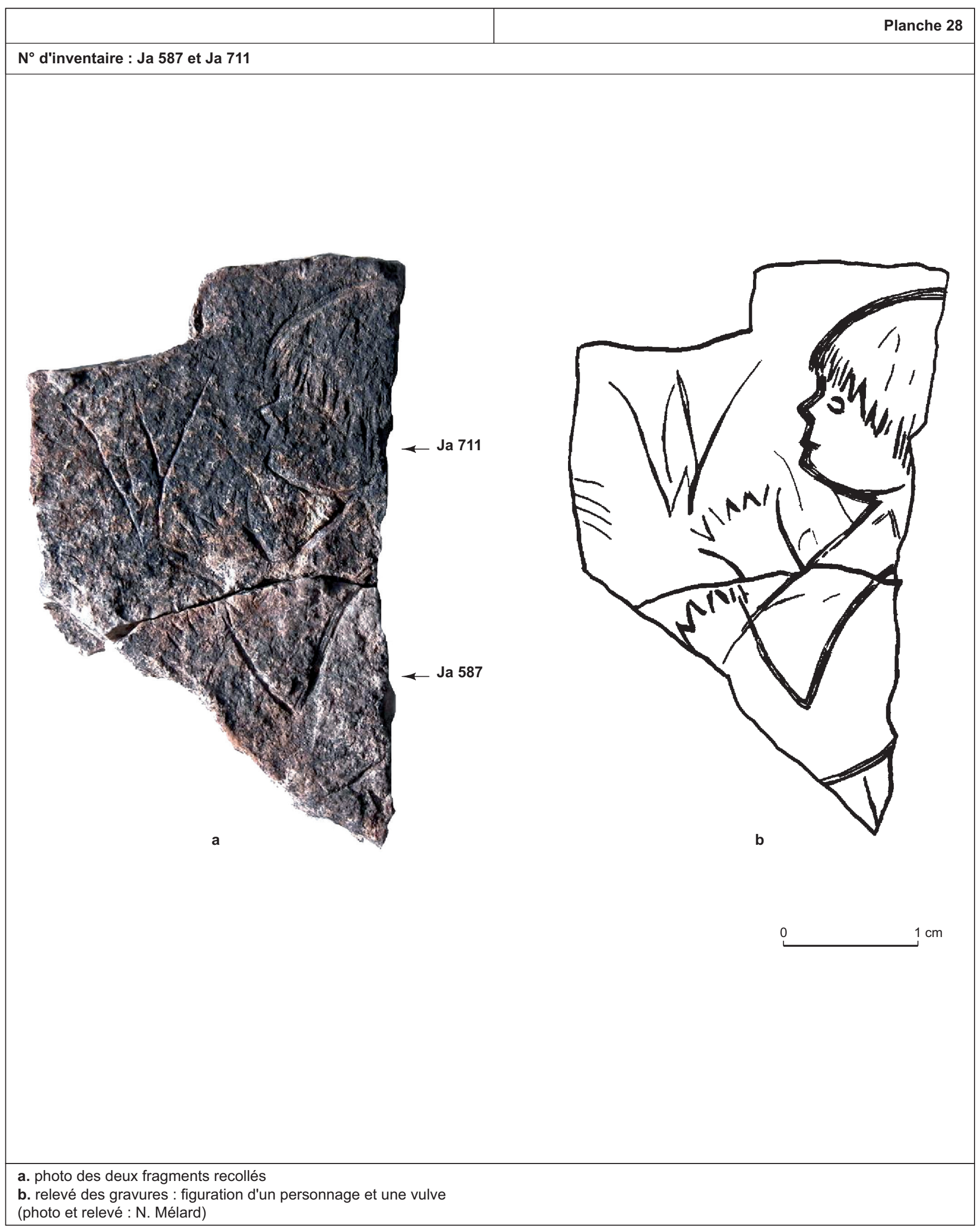




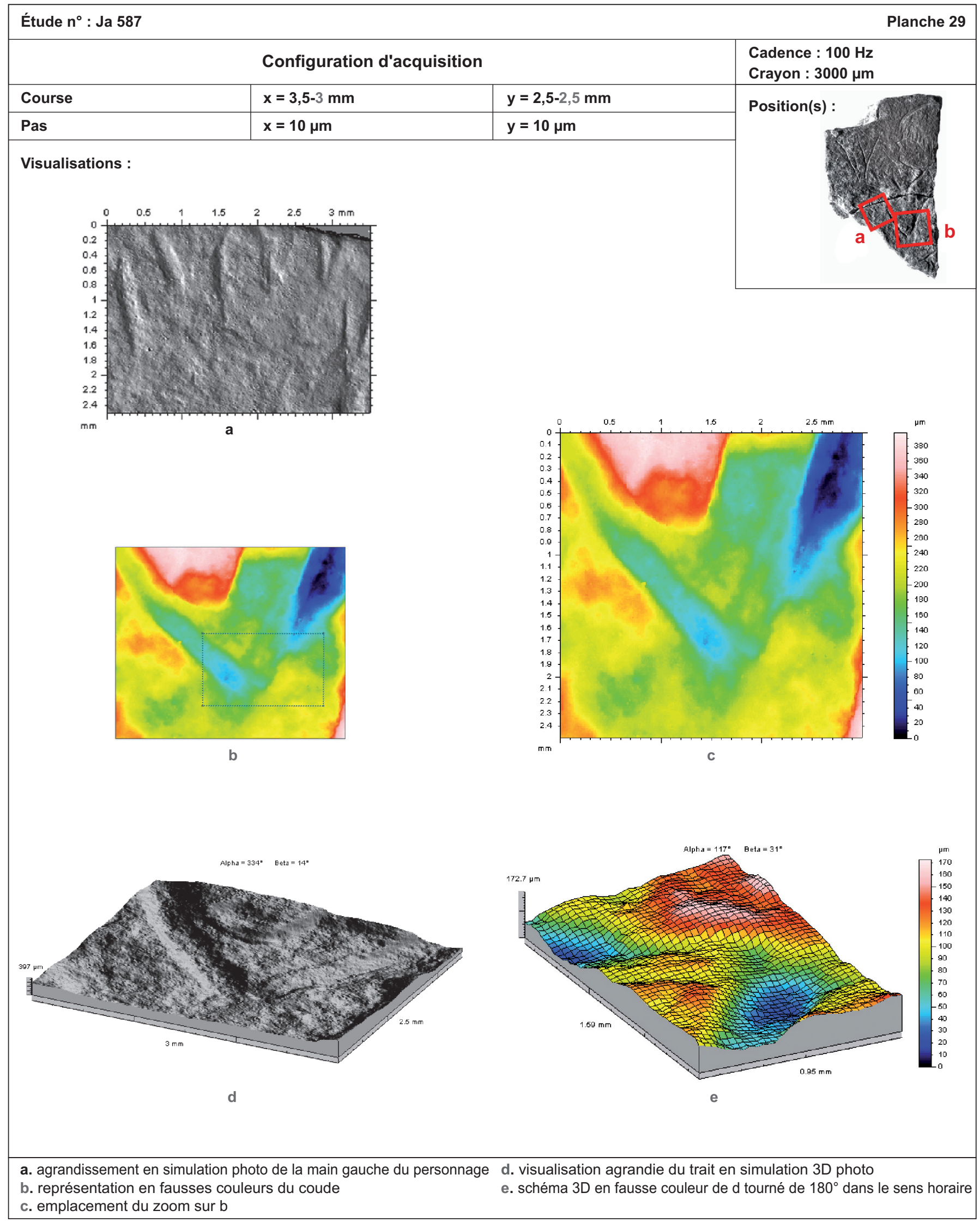




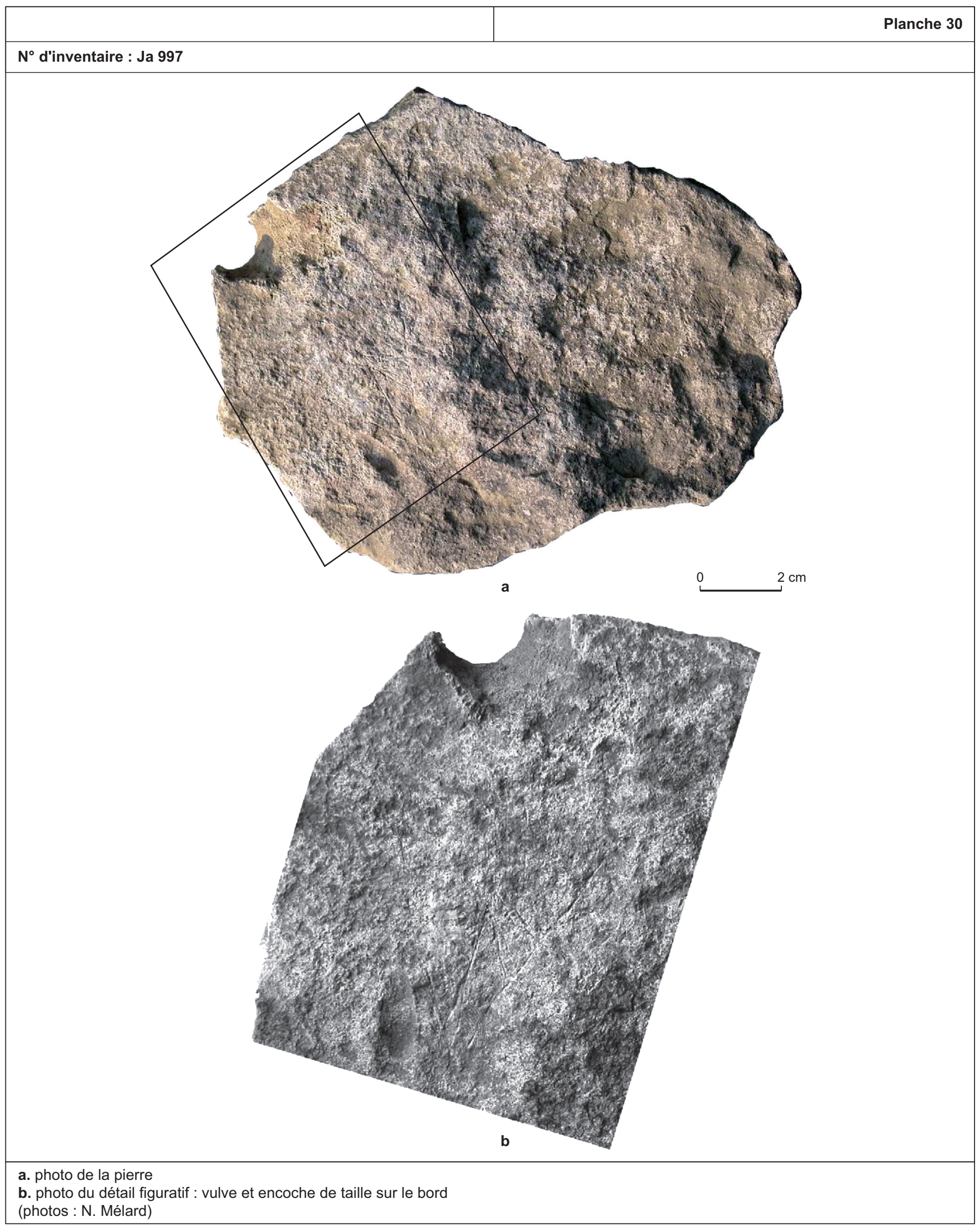




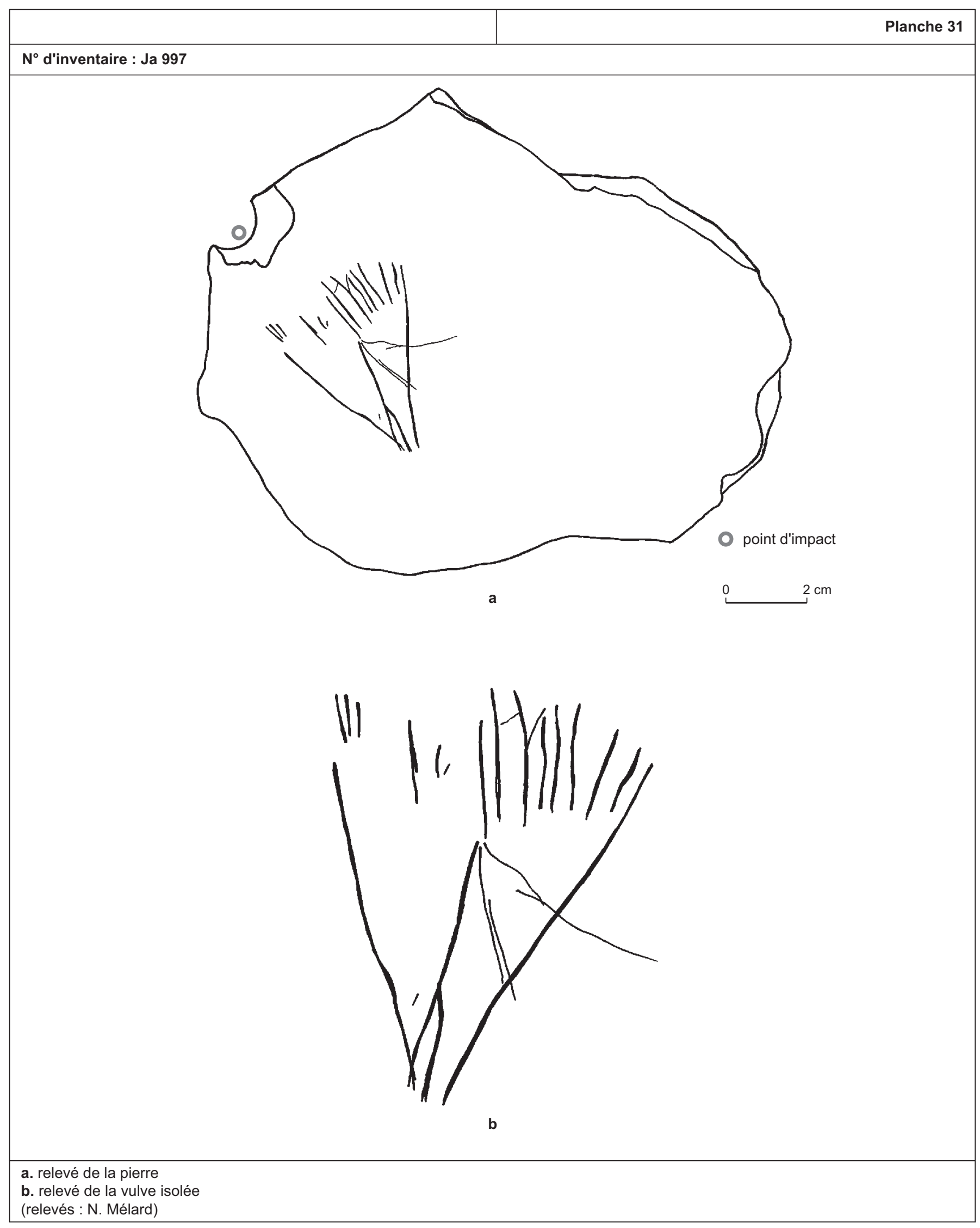




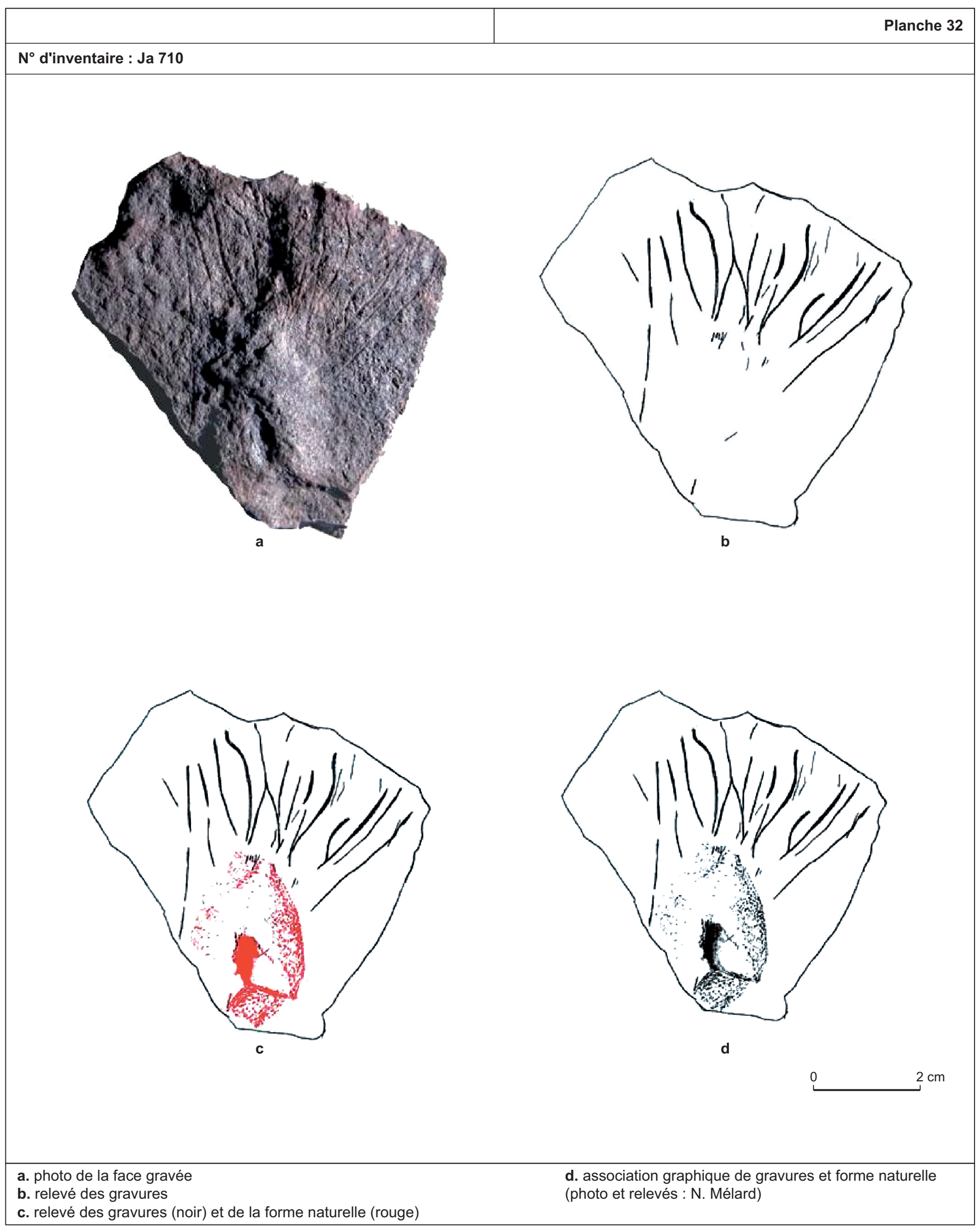




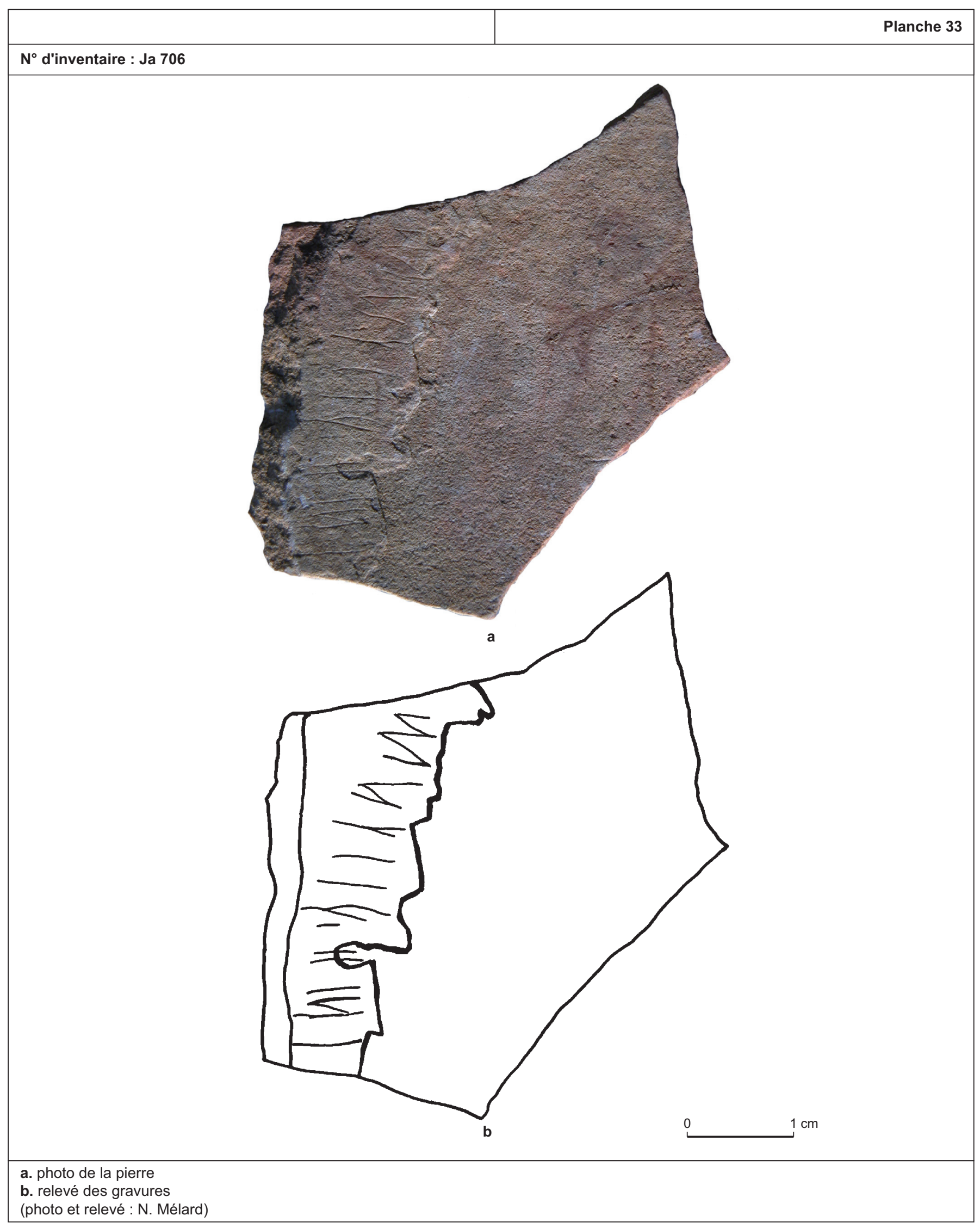




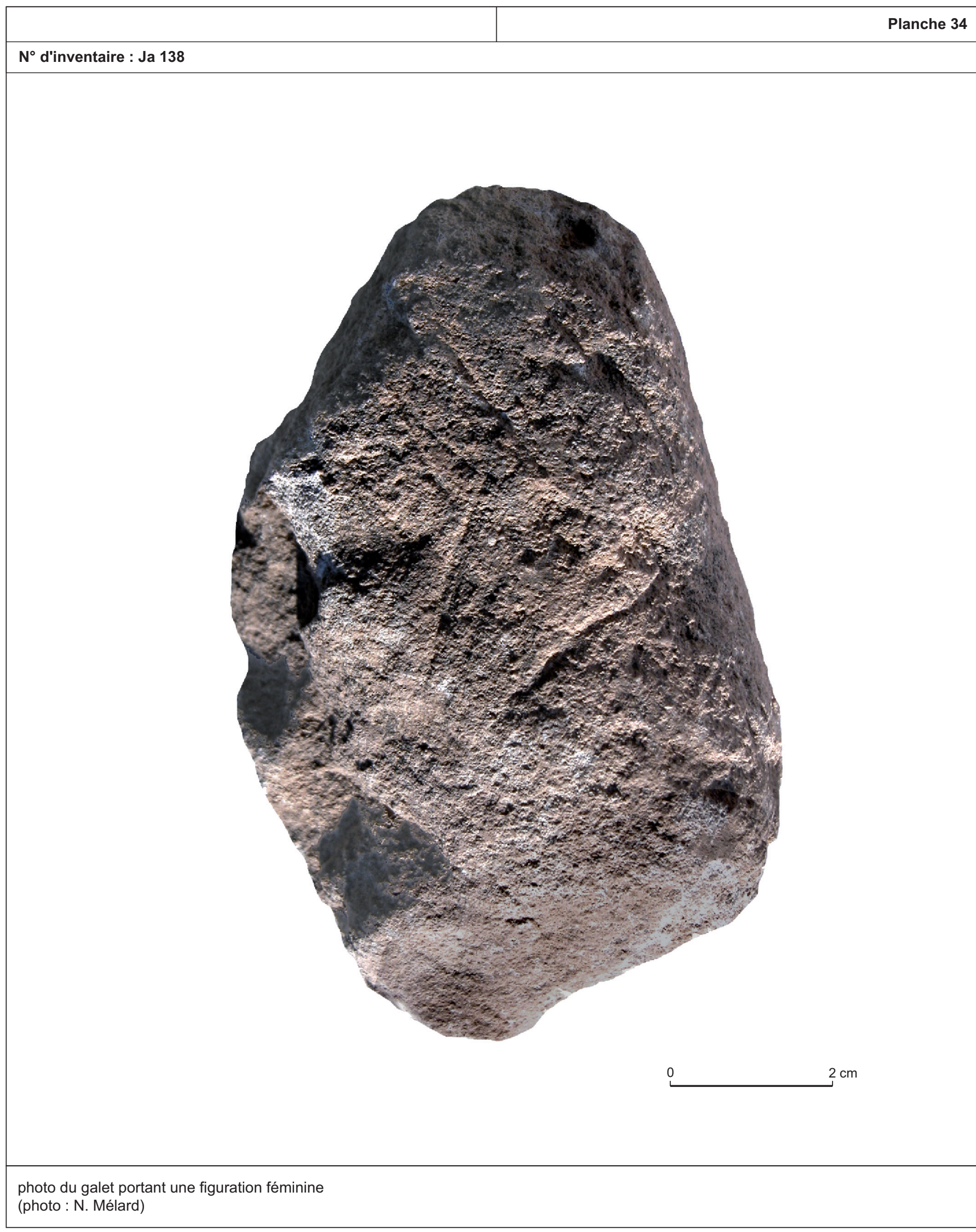




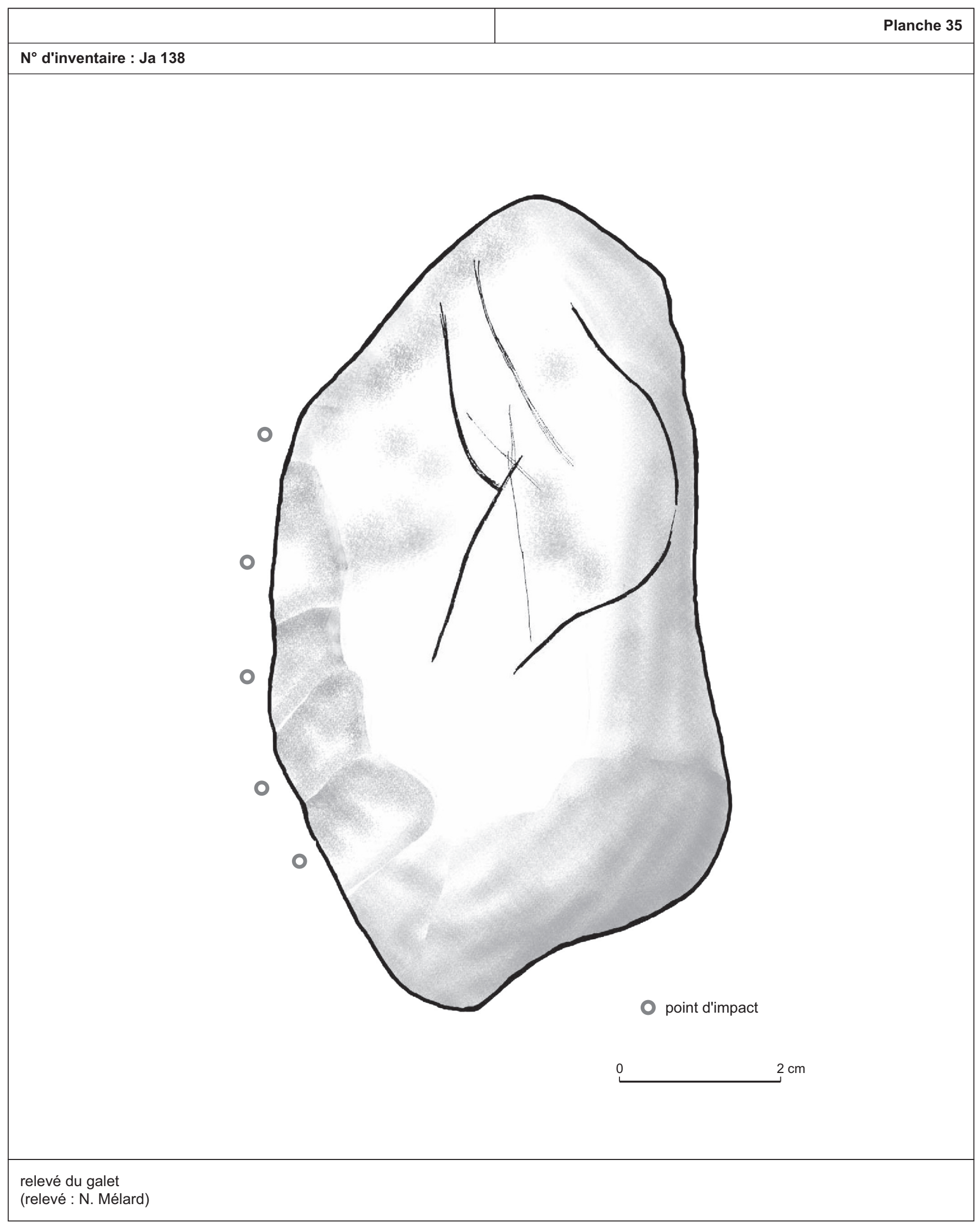




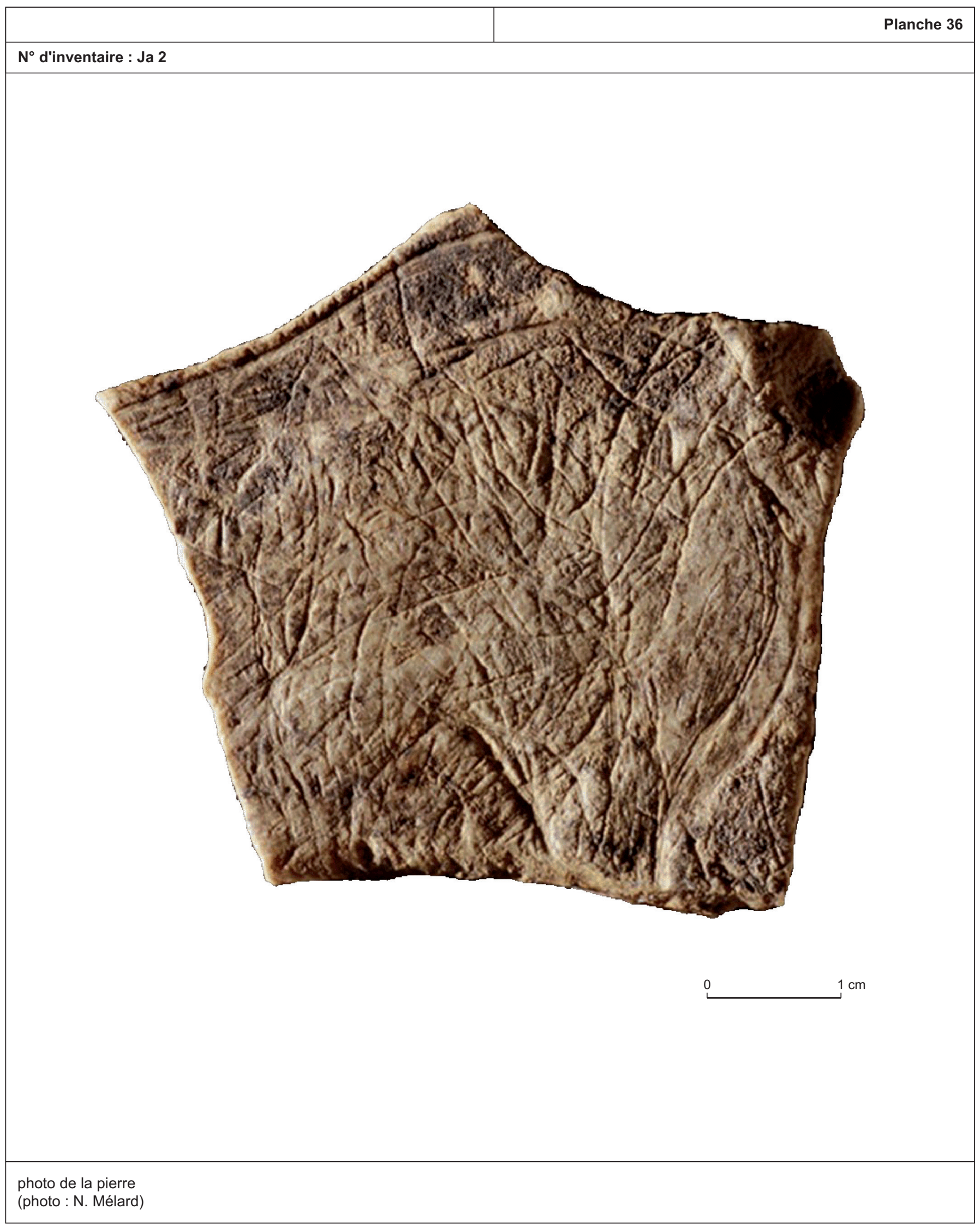




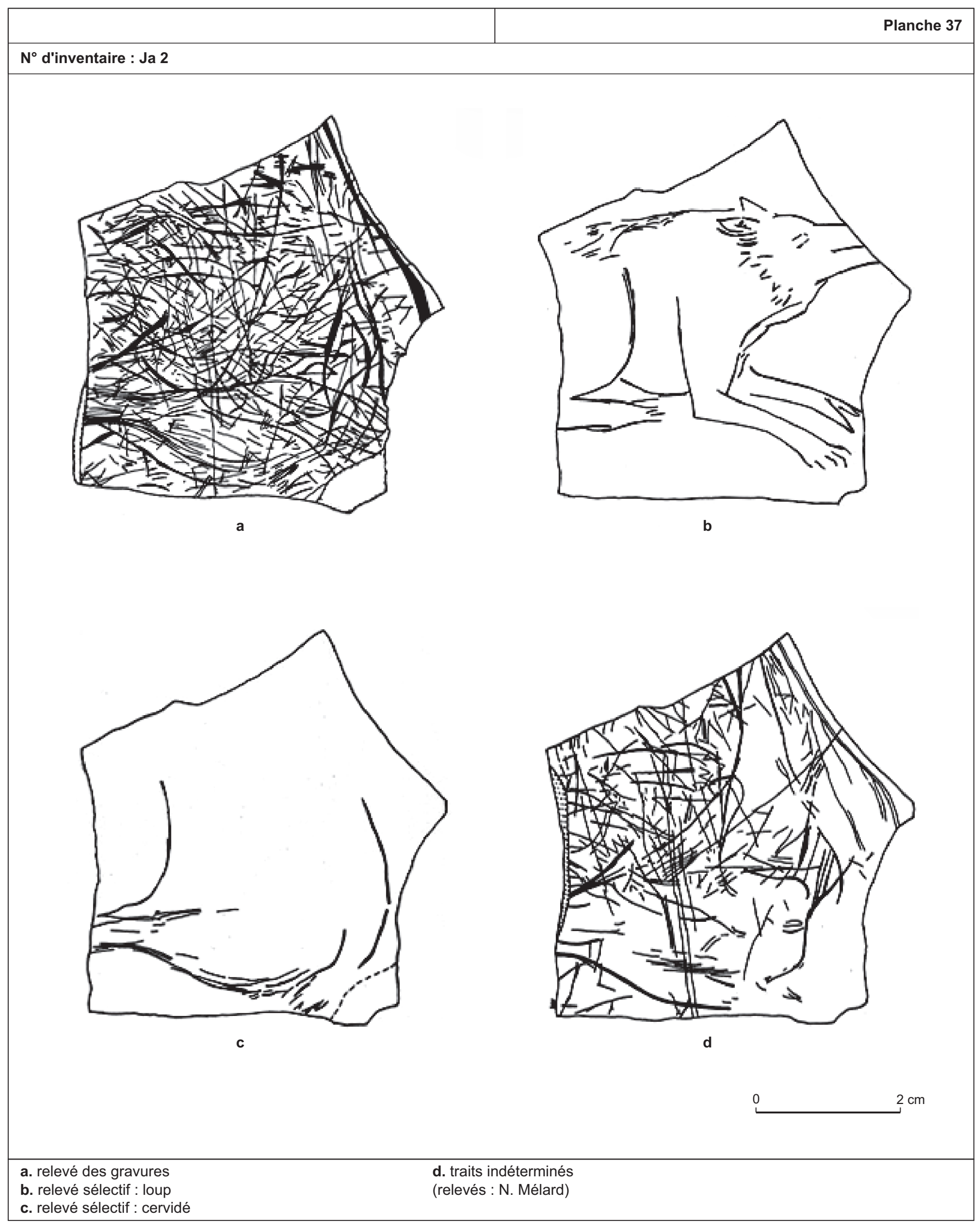




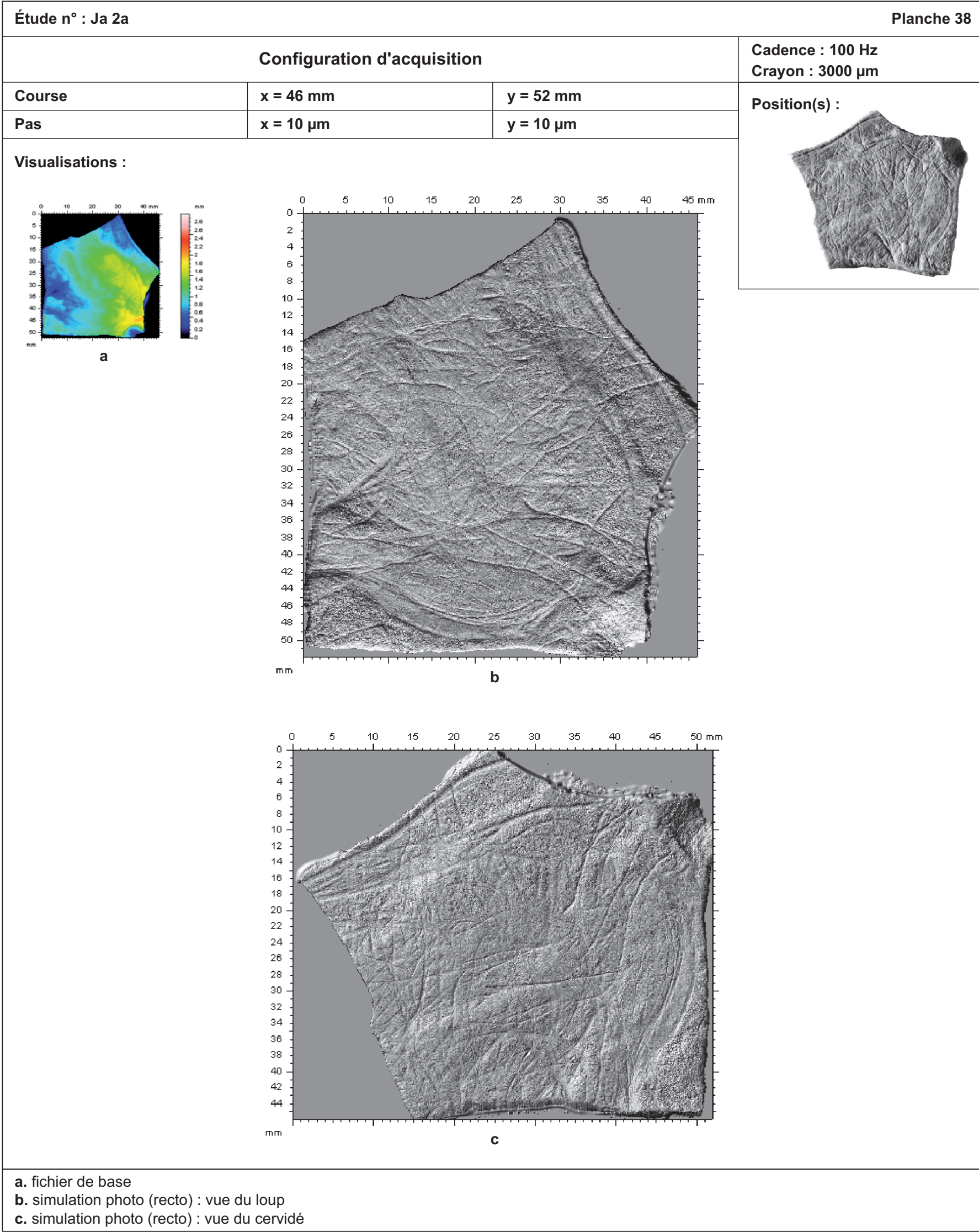




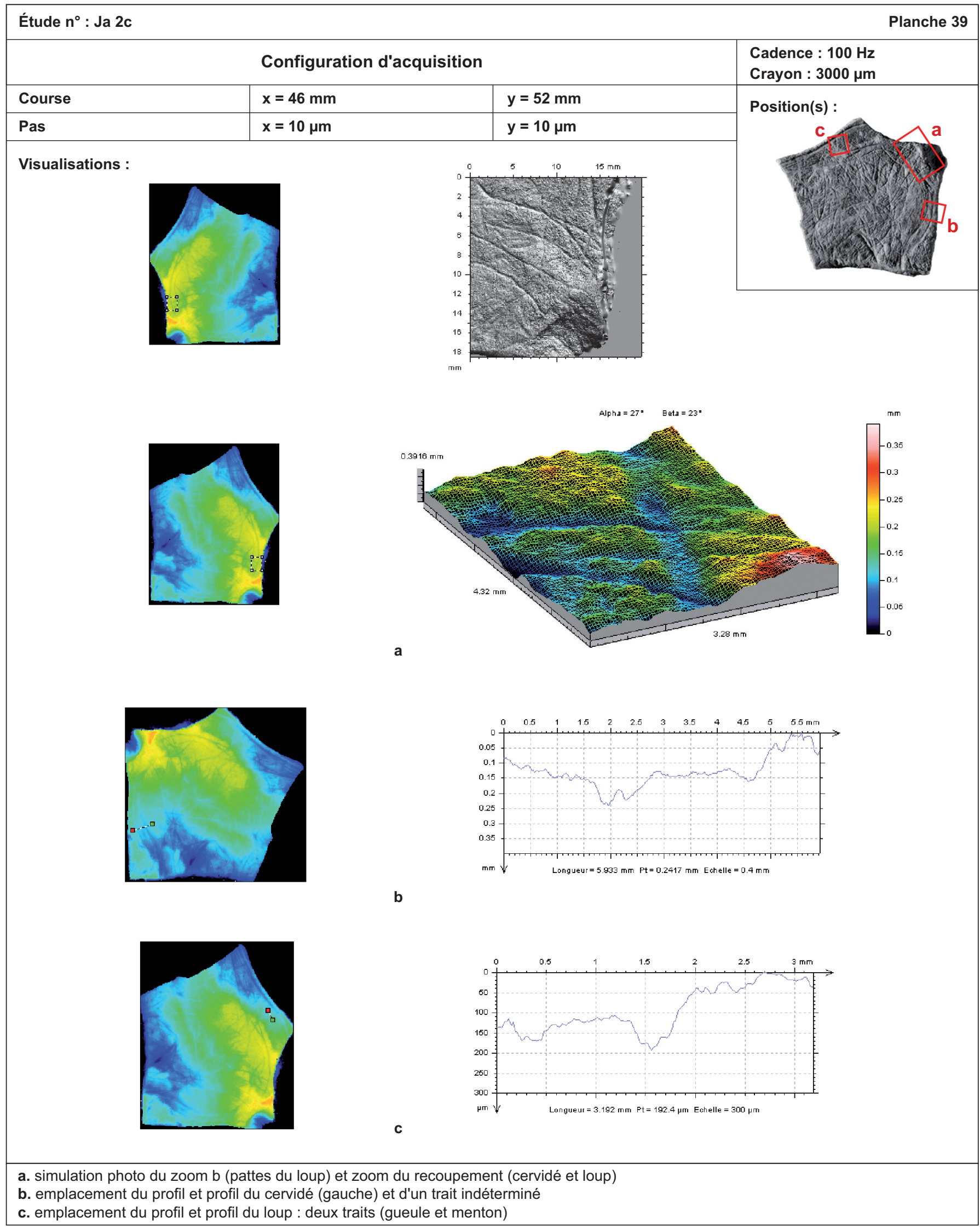




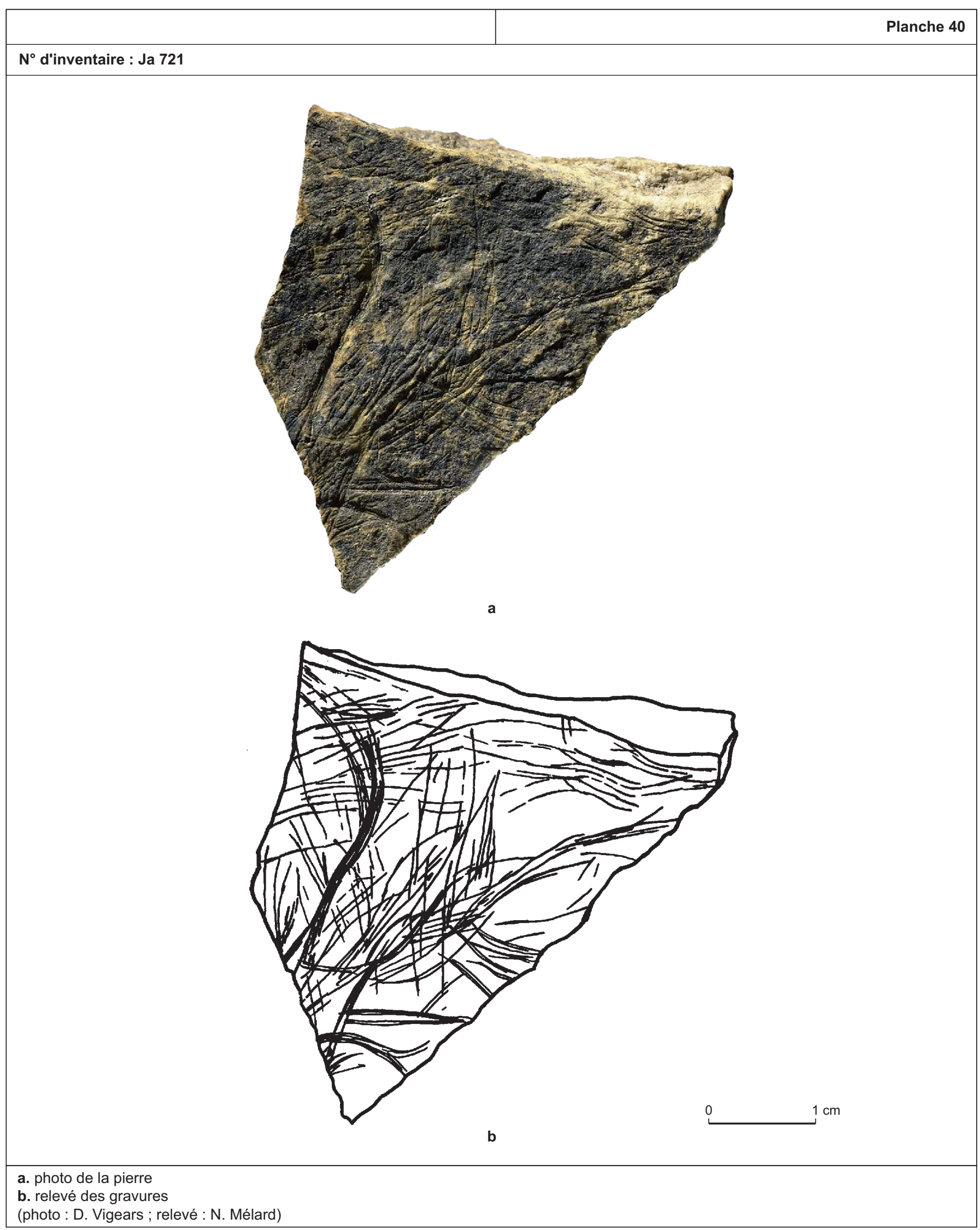




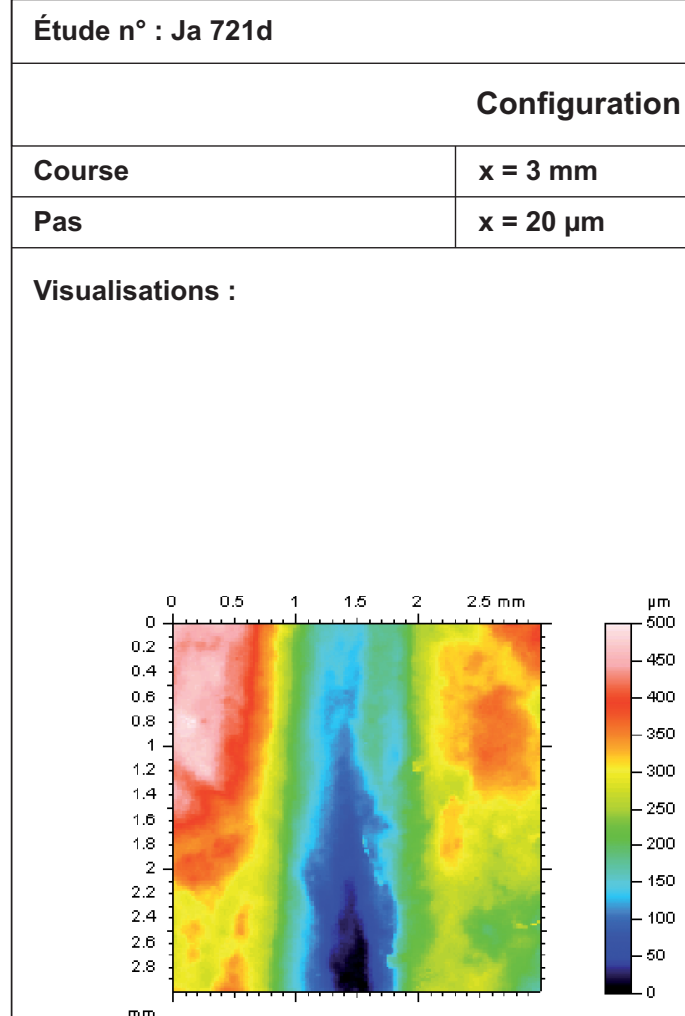

a
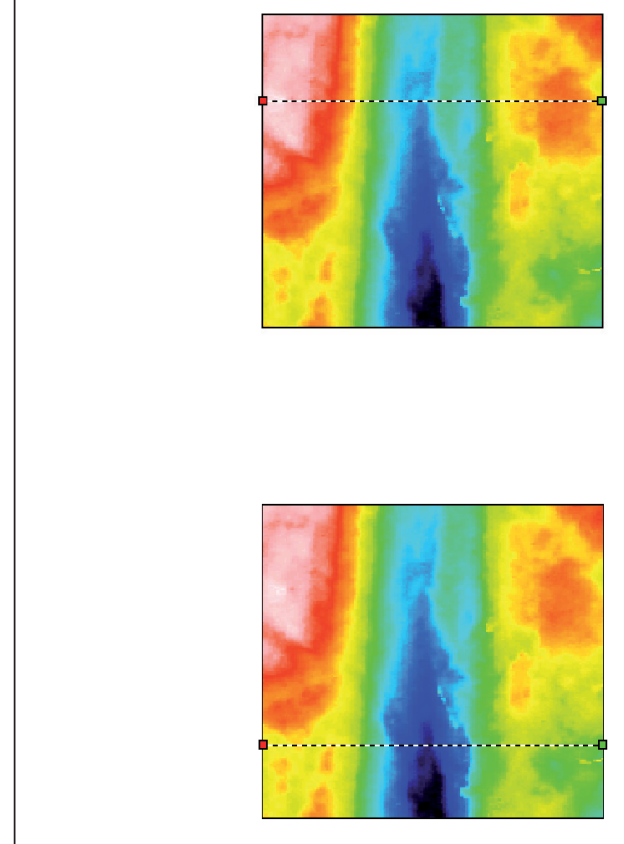

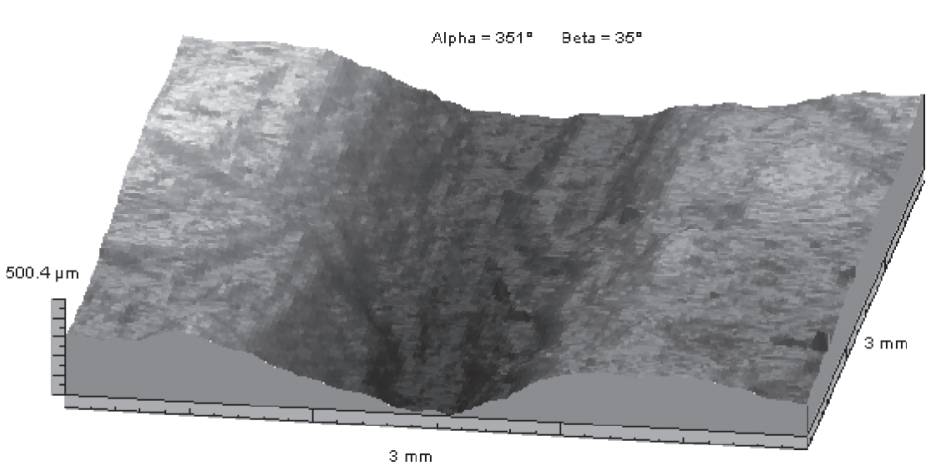

b

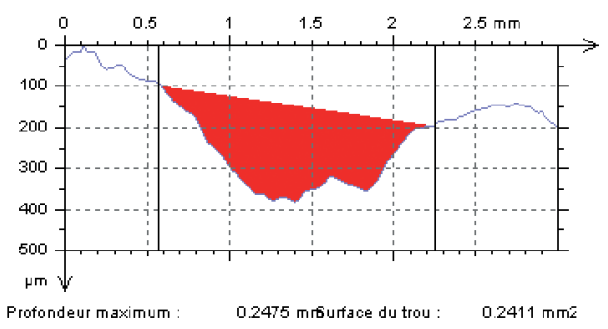

C

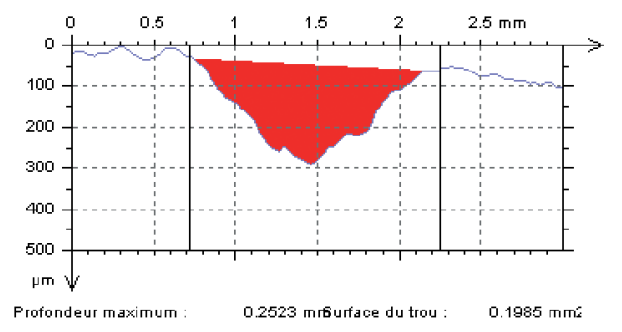

d

c. profil 1 : emplacement et profil

d. profil 2 : emplacement et profil

Frofondeur maximum a. fichier de base

b. simulation photo en bloc 3D

Cadence : $100 \mathrm{~Hz}$ Crayon : $\mathbf{3 0 0 0} \mu \mathrm{m}$

Position(s) :

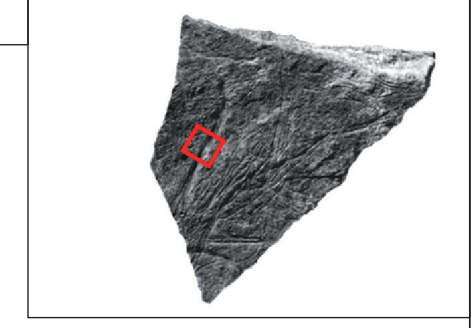

Planche 41

\begin{tabular}{|l|l} 
& $y=3 \mathrm{~mm}$ \\
\hline$y=20 \mu \mathrm{m}$
\end{tabular}




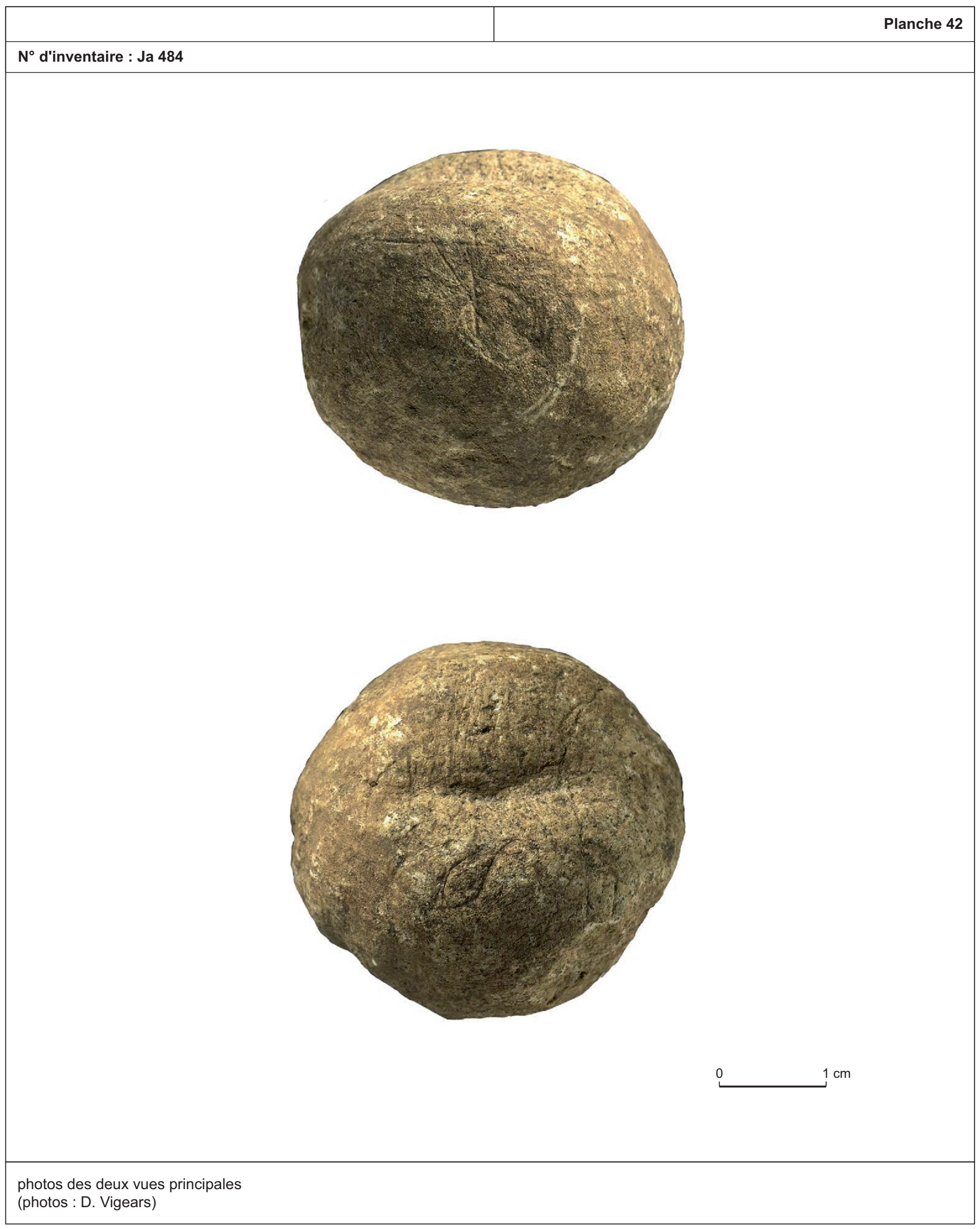




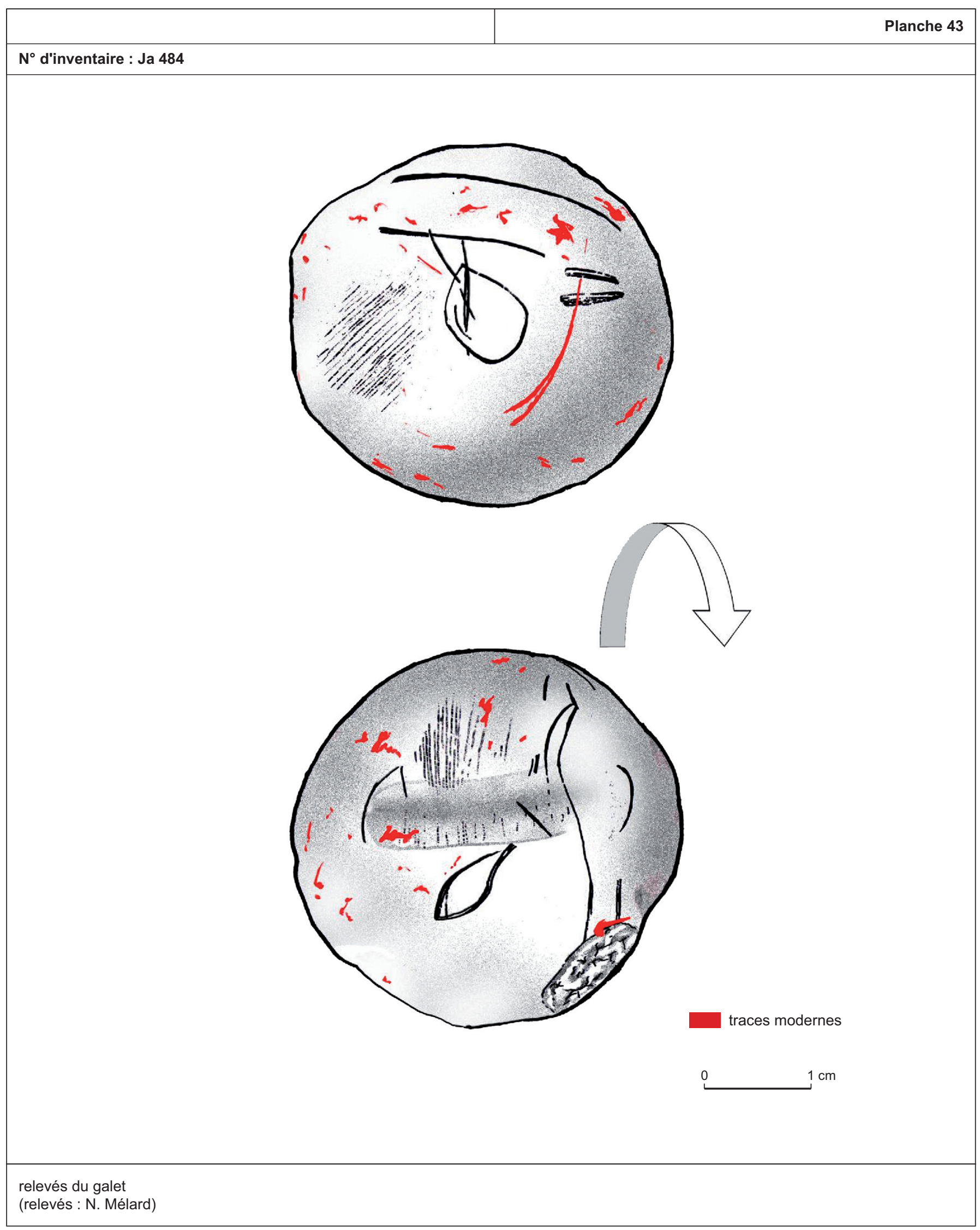




\begin{tabular}{|l|l|l|}
\hline \multicolumn{2}{|l|}{ Étude $\mathrm{n}^{\circ}:$ Ja 484} & \multicolumn{2}{l|}{ Configuration d'acquisition } \\
\hline \multicolumn{3}{|l|}{} \\
\hline Course & $x=8-8-5 \mathrm{~mm}$ & $\mathrm{y}=8-8-5 \mathrm{~mm}$ \\
\hline Pas & $\mathrm{x}=10-100-5 \mu \mathrm{m}$ & $\mathrm{y}=10-100-5 \mu \mathrm{m}$ \\
\hline
\end{tabular}

Visualisations :

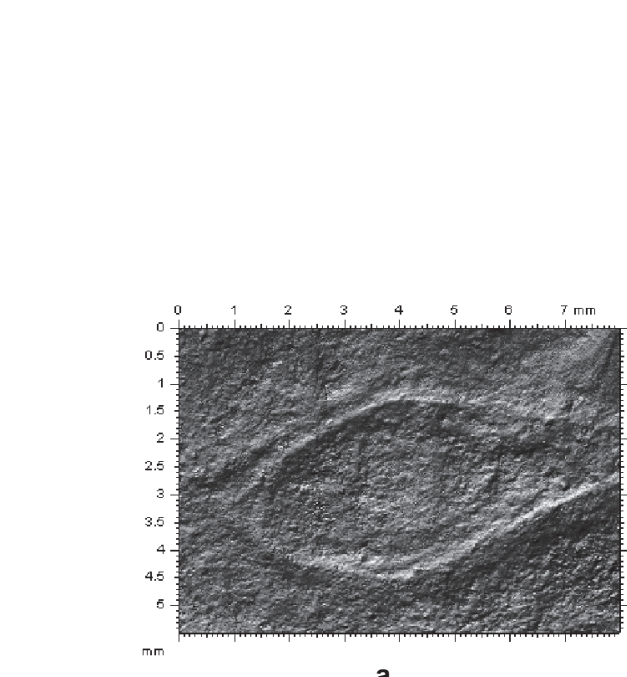

a

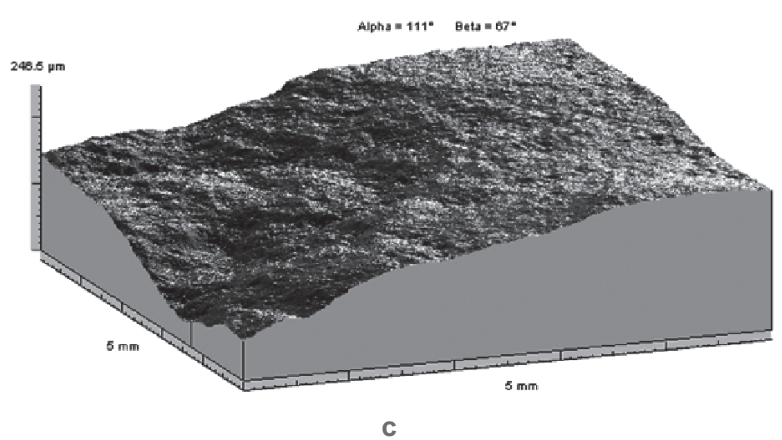

Planche 44

Cadence : $100 \mathrm{~Hz}$ Crayon : $\mathbf{3 0 0 0} \boldsymbol{\mu m}$

Position(s) :

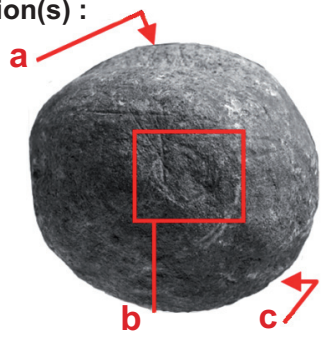

Alpha $=305^{\circ} \quad$ Bsta $=30^{\circ}$

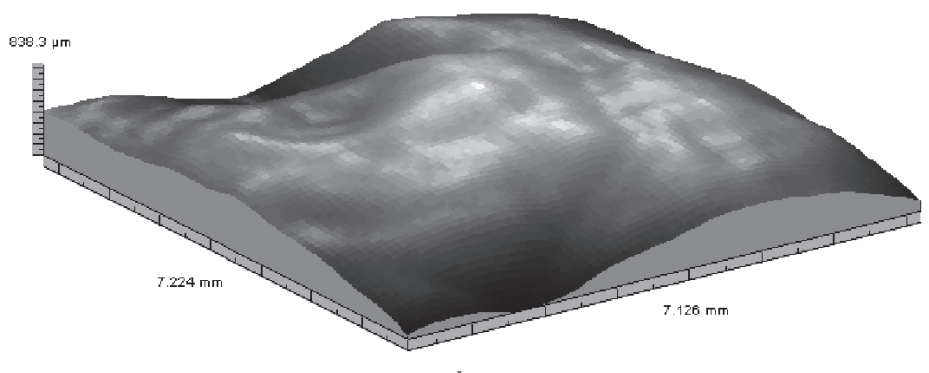

b

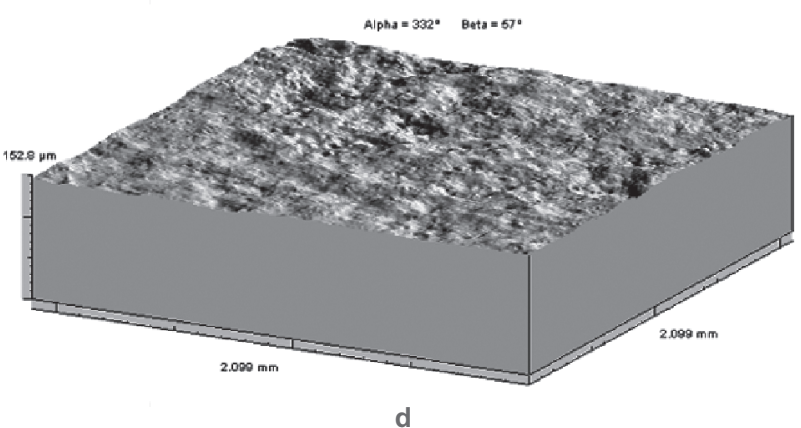

d
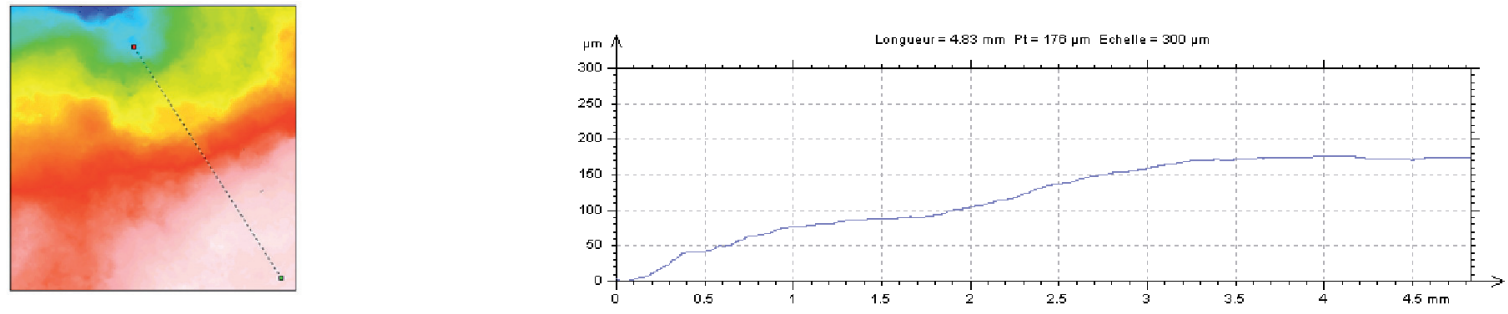

a. détail de motif gravé en simulation photo (" oeil isolé a »)

b. détail de motif gravé en simulation photo en bloc 3D (« oeil isolé b »)

c. détail de zone d'écrasement d. zone c filtrée (filtre de rugosité gaussien $0,08 \mathrm{~mm}$ ) e. profil de la zone c (emplacement et profil) 


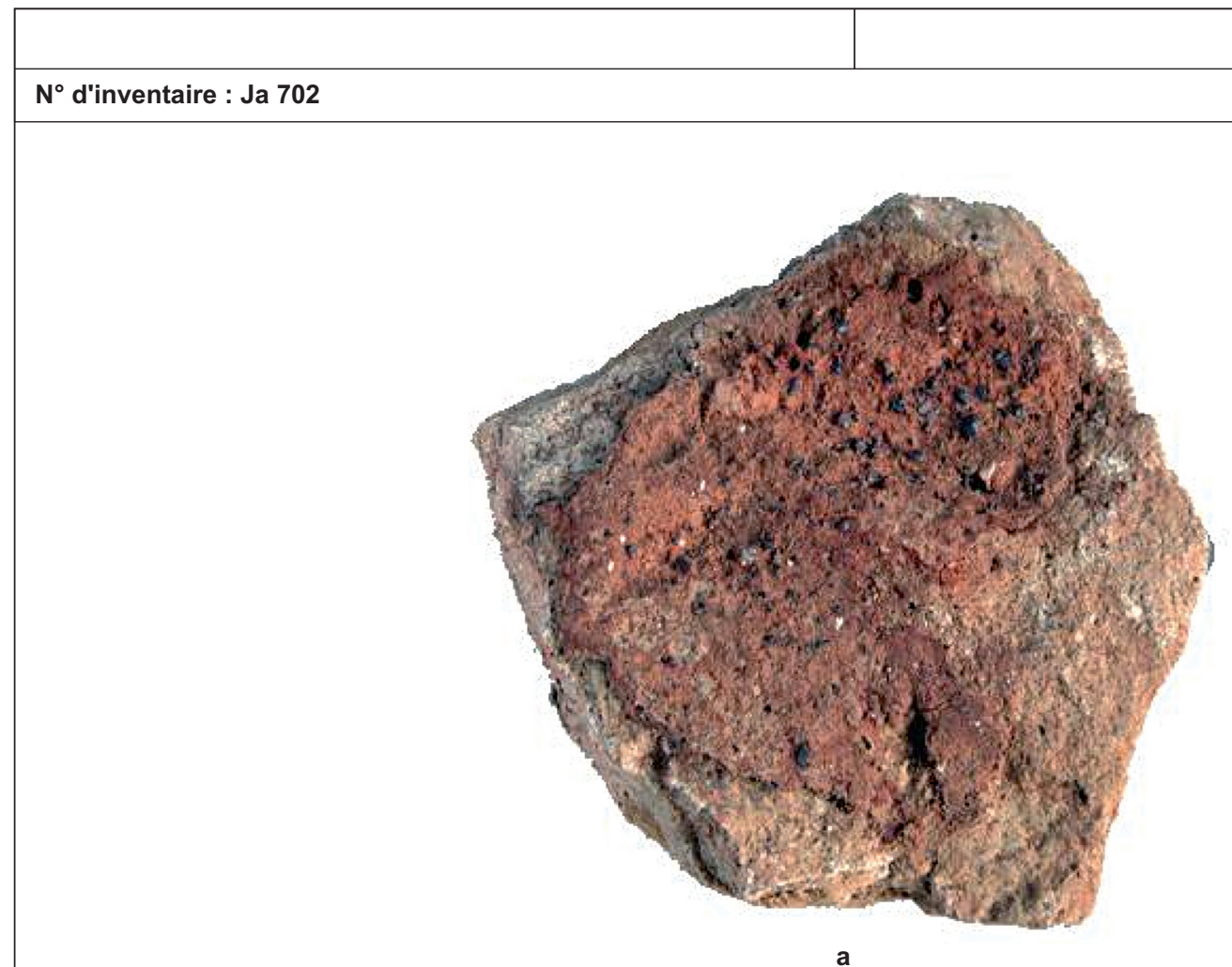

Planche 45

$\mathrm{N}^{\circ}$ d'inventaire : Ja 702

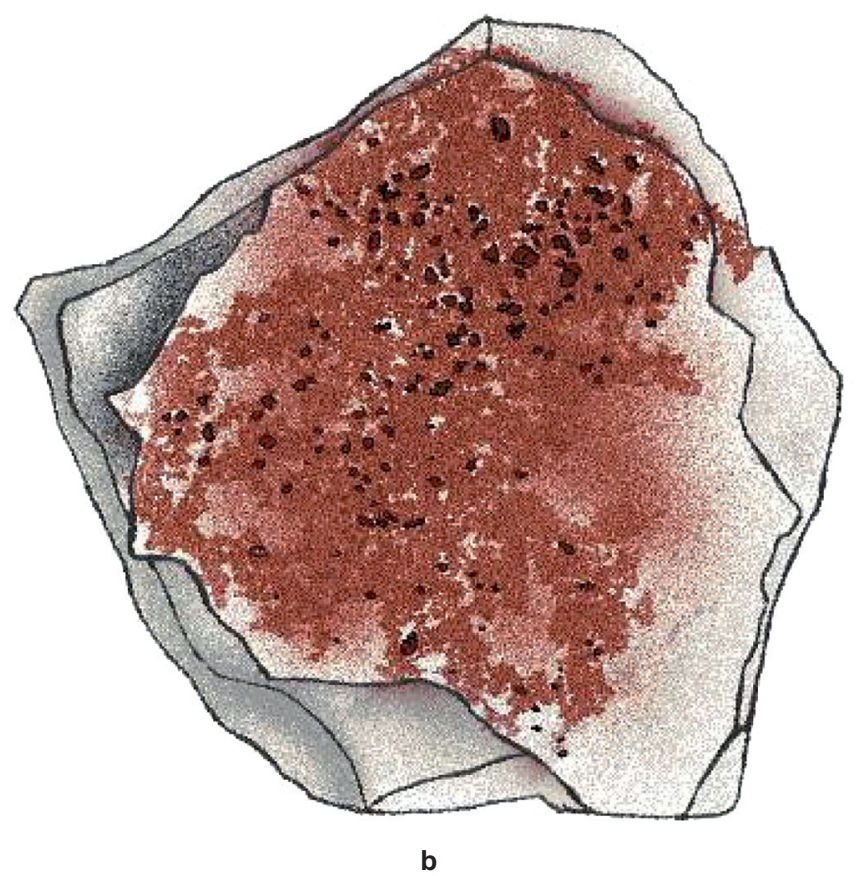

0 $2 \mathrm{~cm}$

a. photo de la pierre avec encroûtement rouge b. relevé de la pierre

(photo et relevé : N. Mélard) 


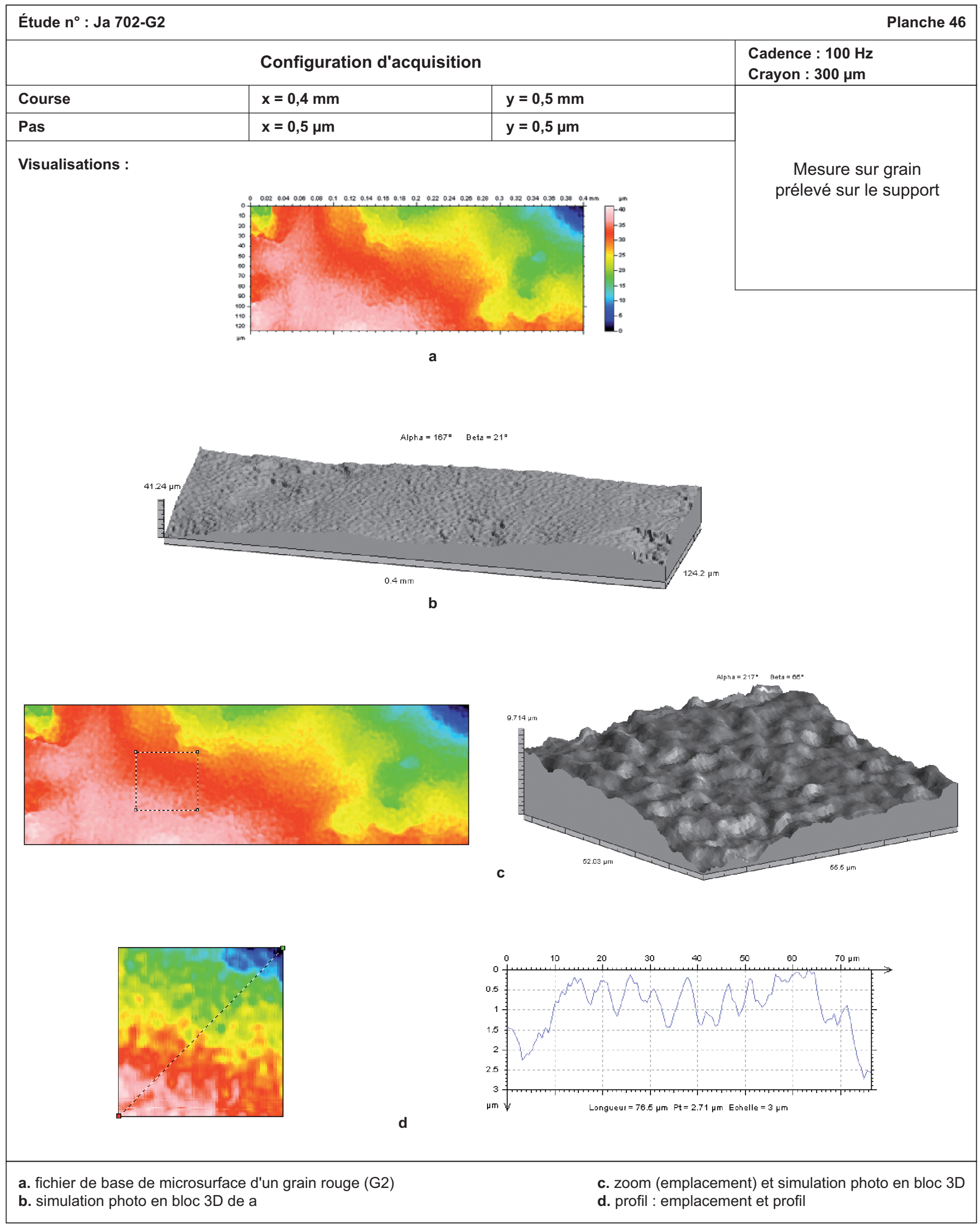




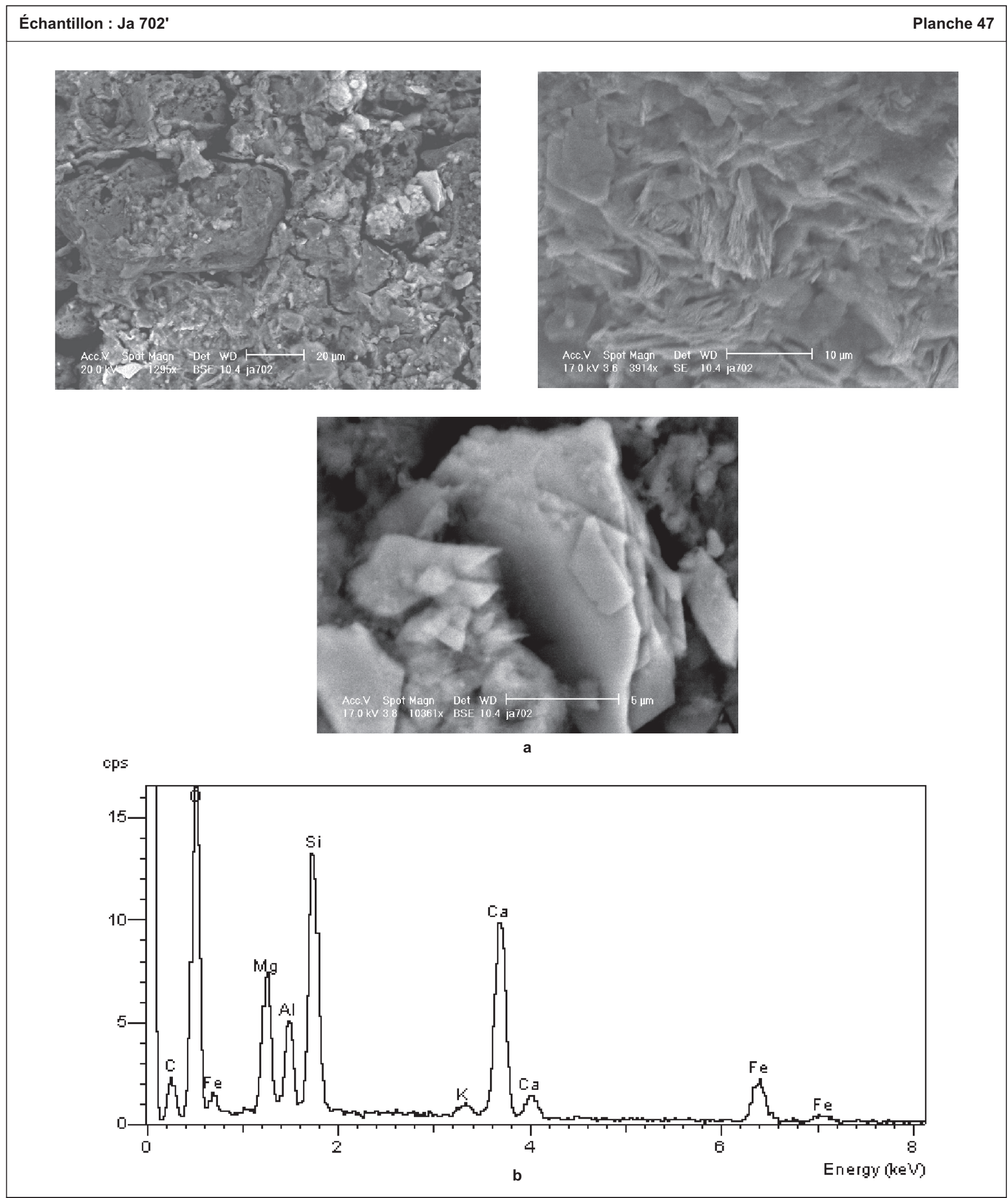

a. images : ensemble (en haut à droite) et oxydes de fer en forme de feuillets (en haut à gauche : x 4000 ; en bas : x 10 400) b. spectre : ensemble du pigment (dolomite, oxyde de fer) 


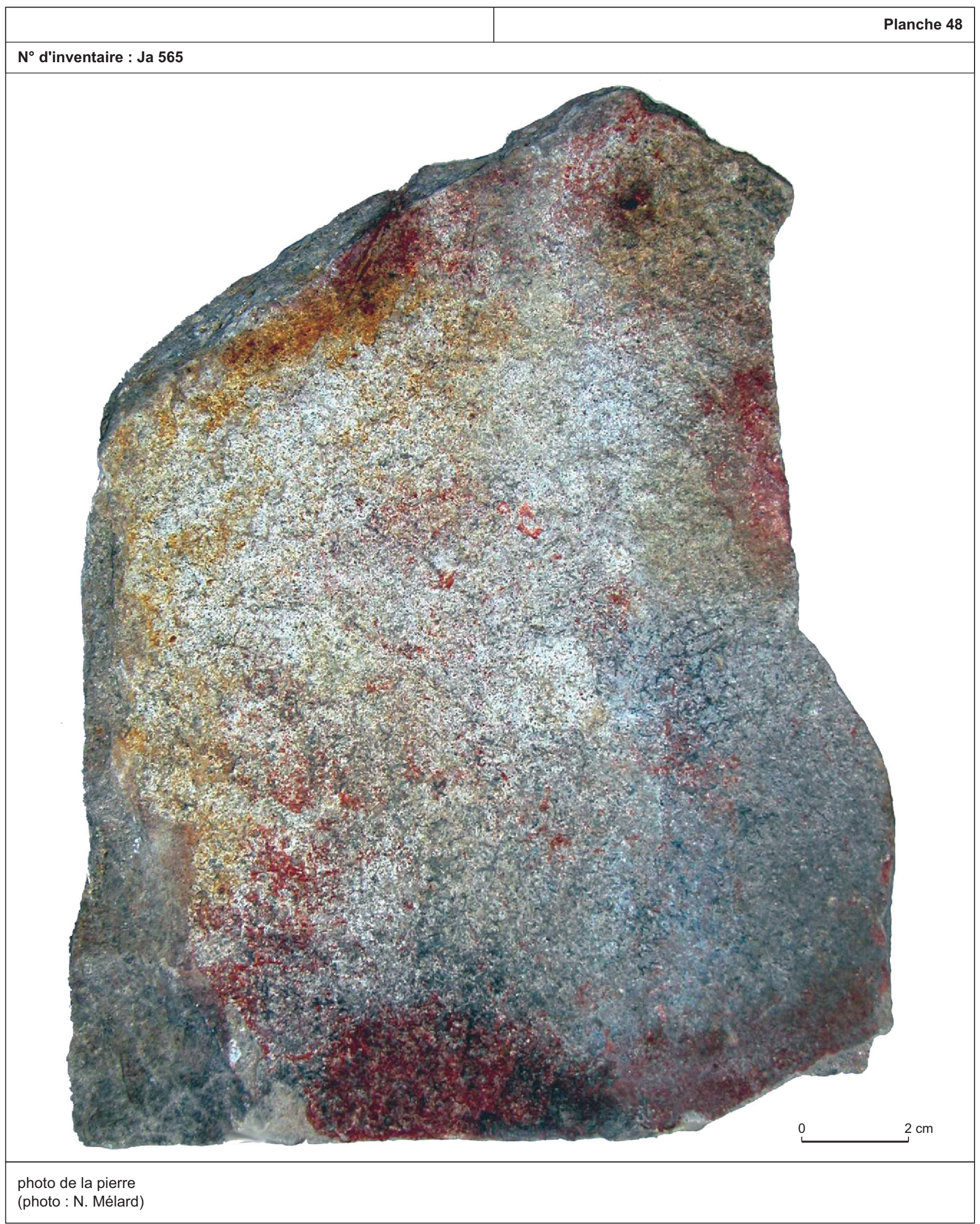




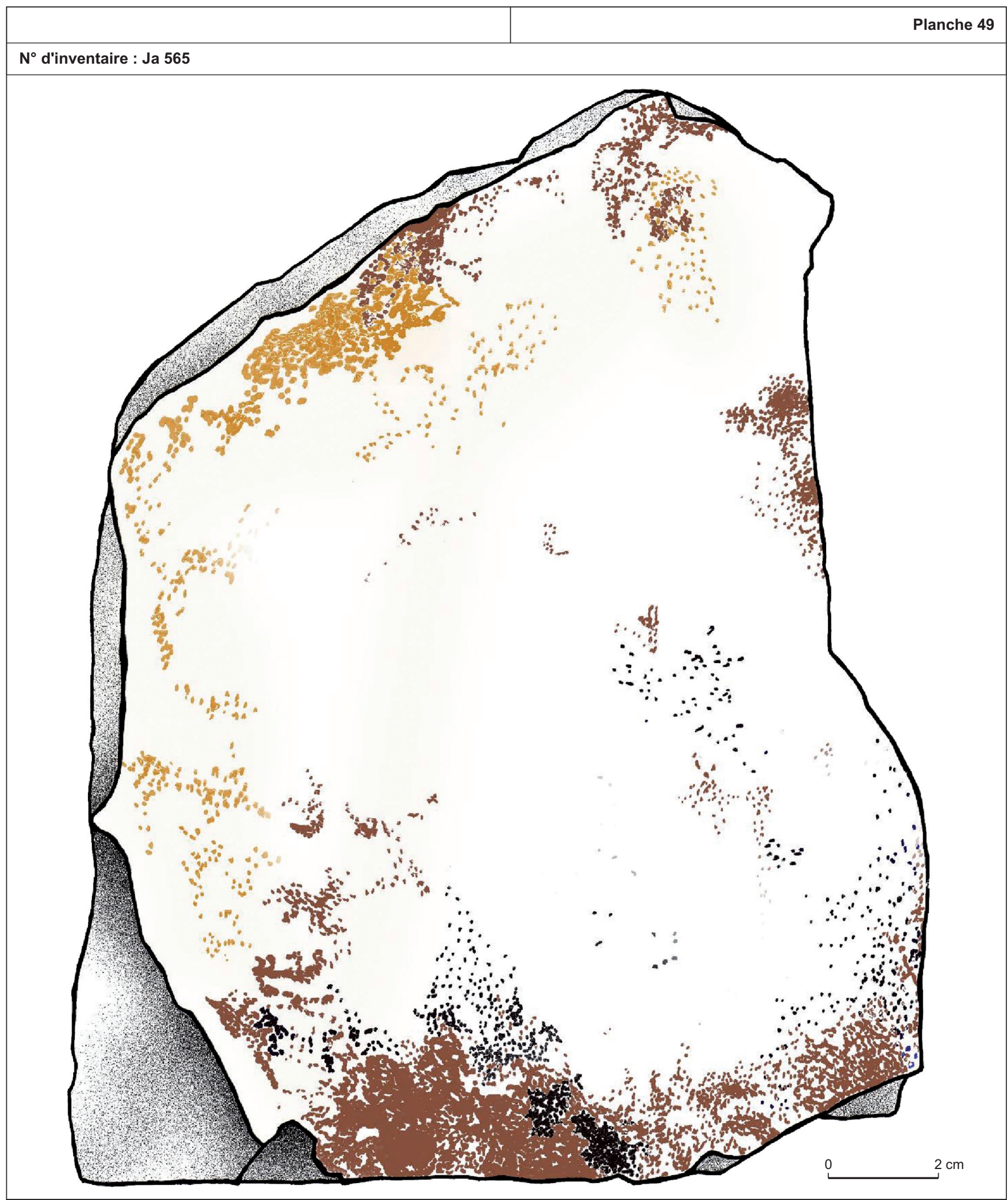

relevé de la face colorée

(relevé : N. Mélard) 


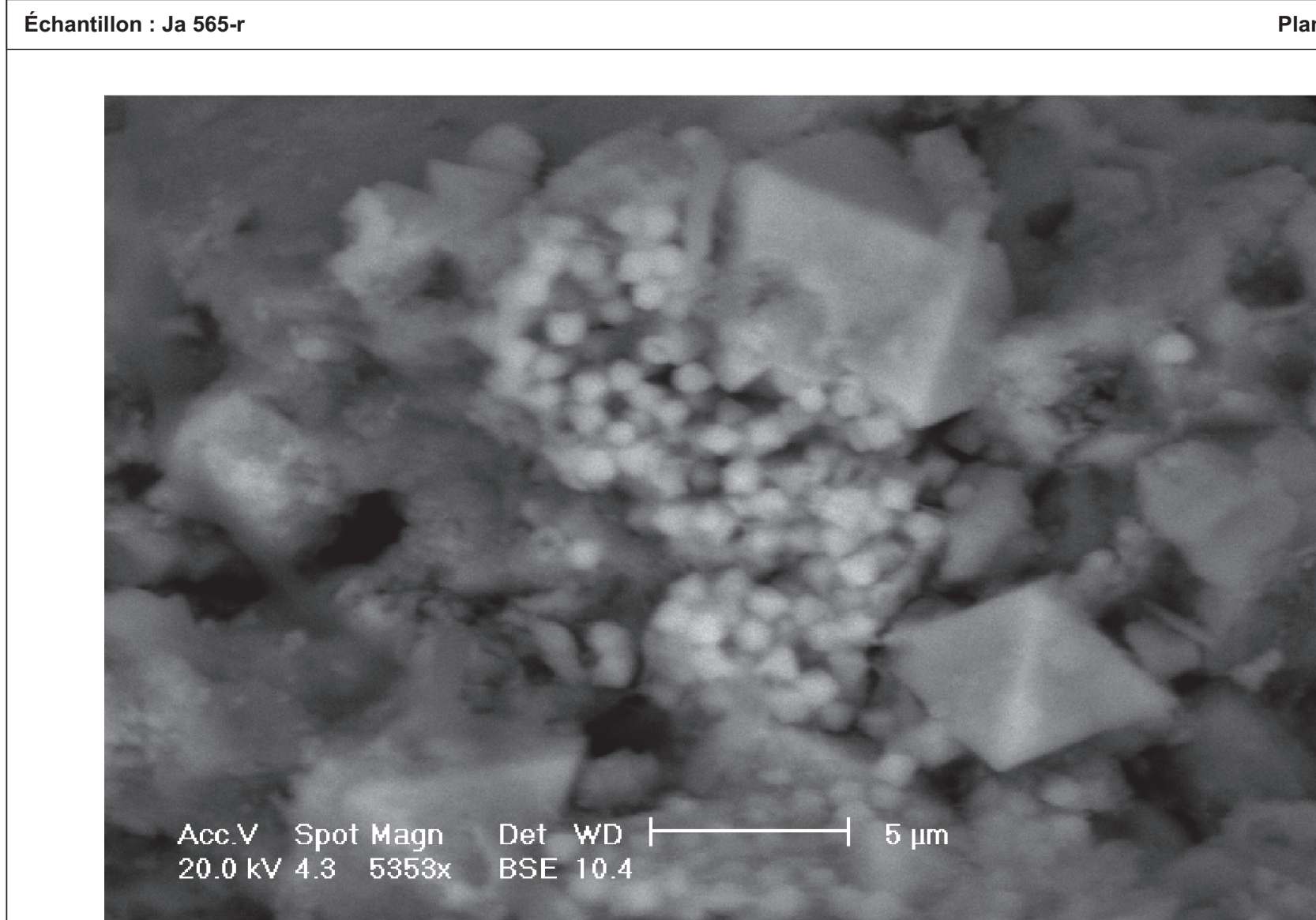

a

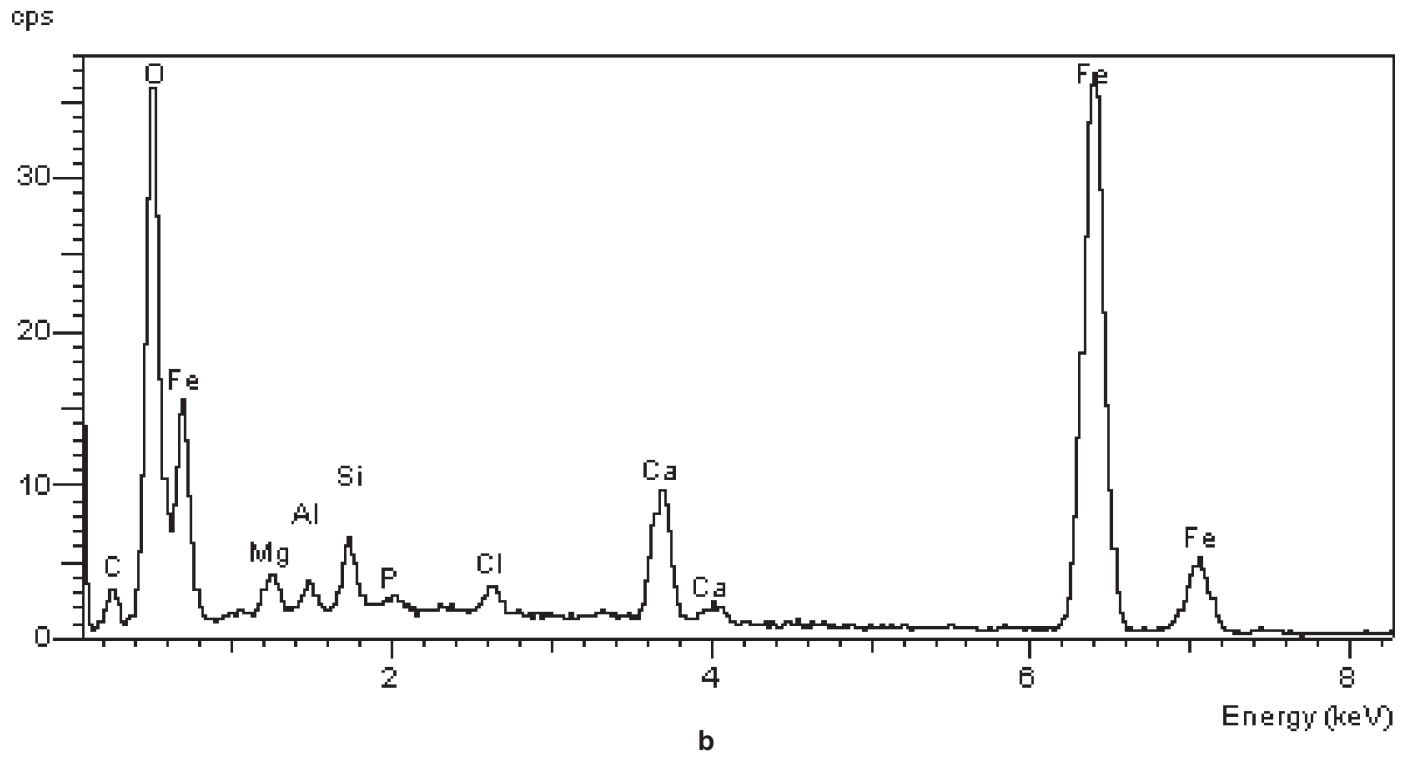

a. image : assemblage d'oxydes de fer en forme de billes et d'octaèdres (x 5300$)$ b. spectre : analyse pointée sur les petits grains $(\mathrm{FeO})$ 


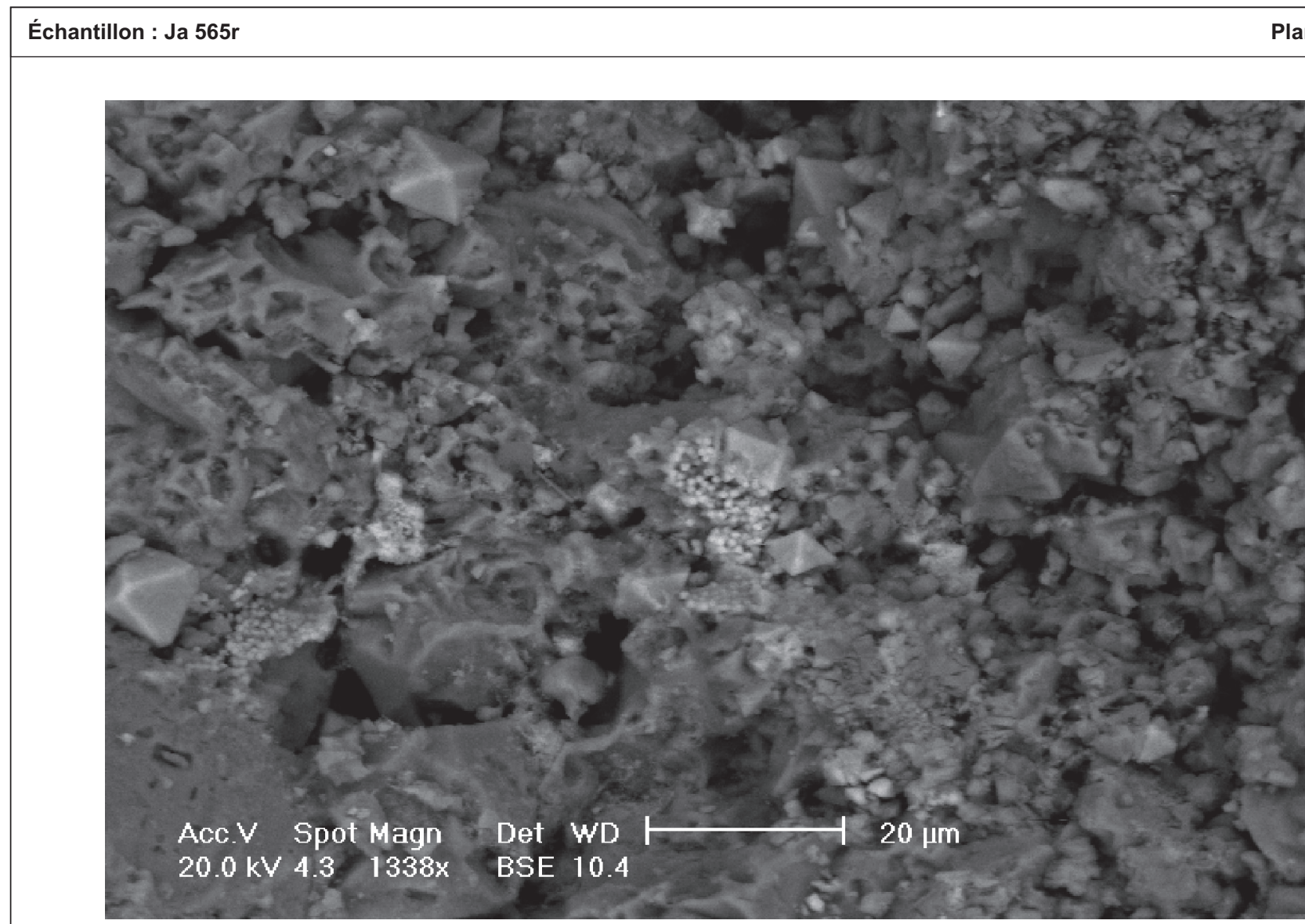

a

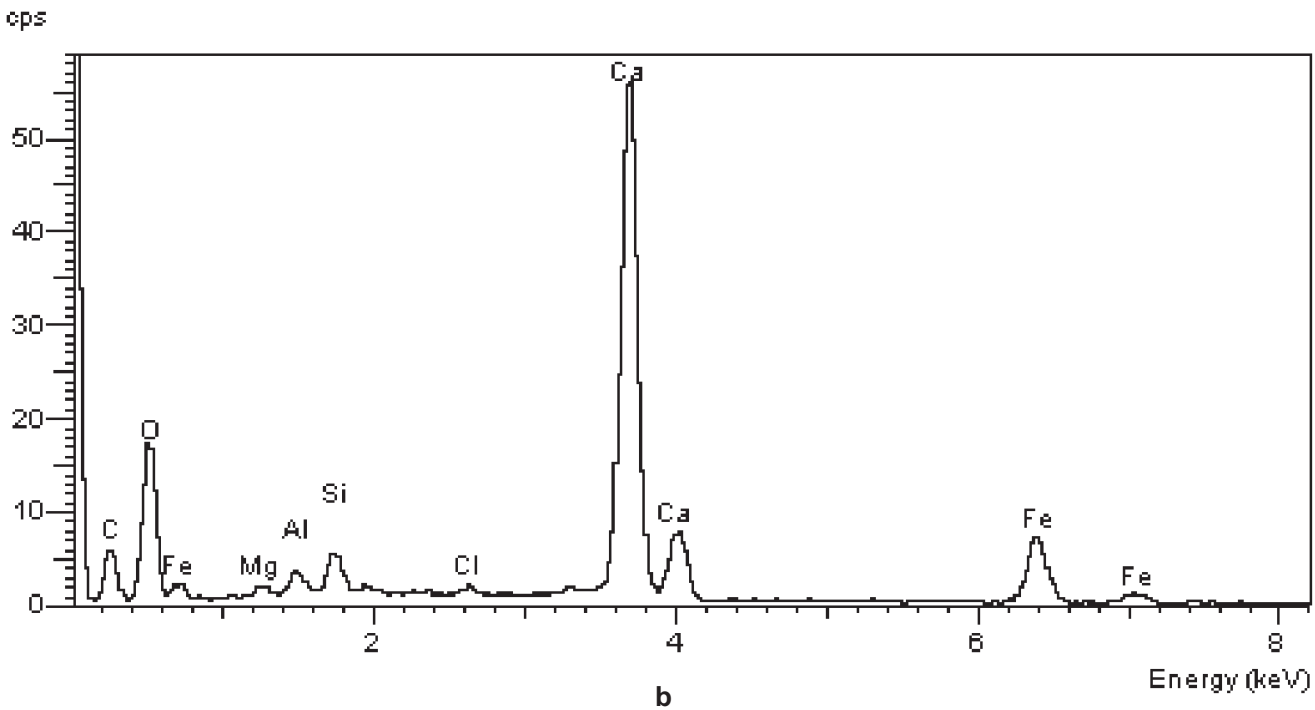

a. image : image MEB (BSE), ensemble de pigment rouge (oxydes de fer, argiles et dolomite) b. spectre : analyse de l'ensemble 


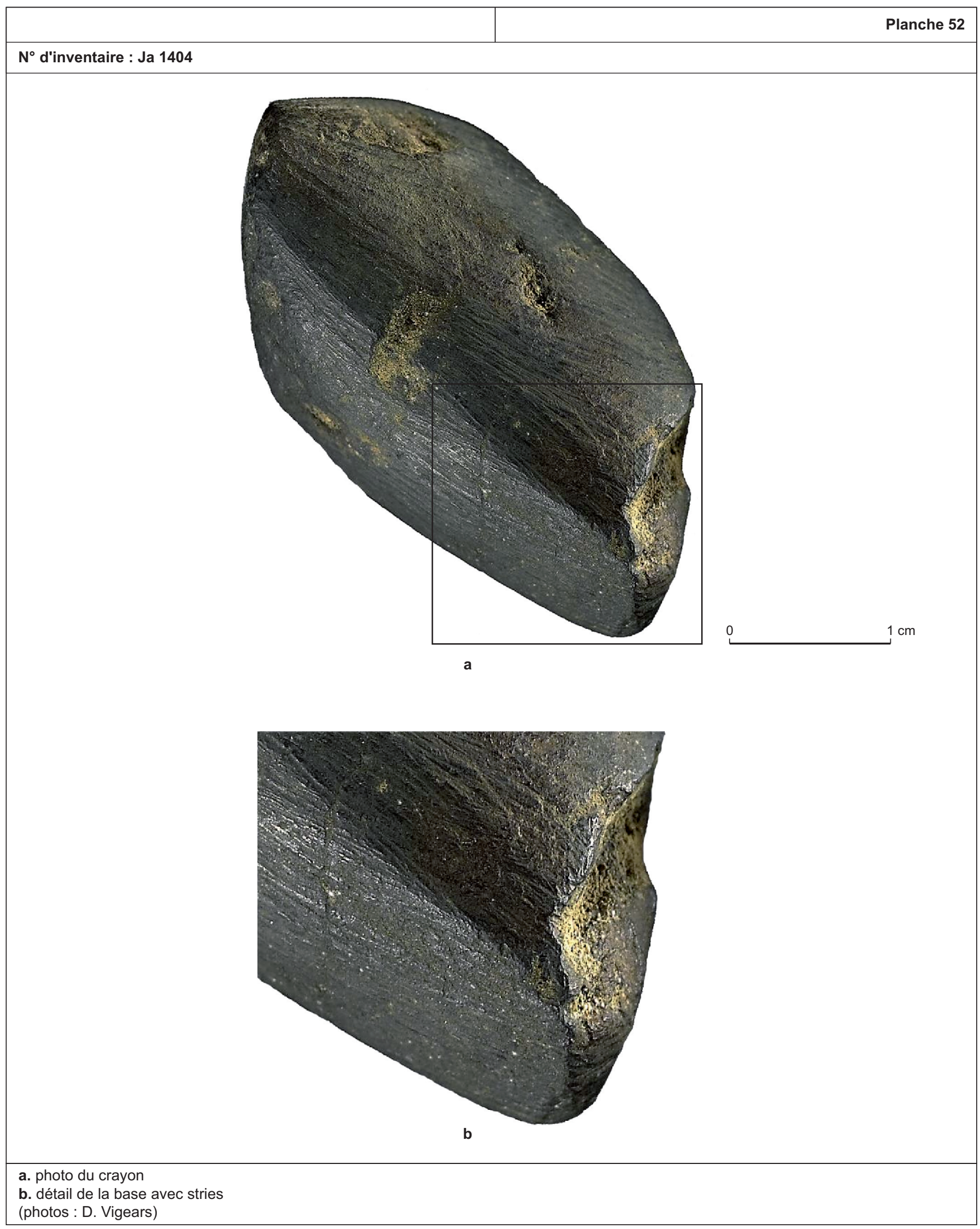




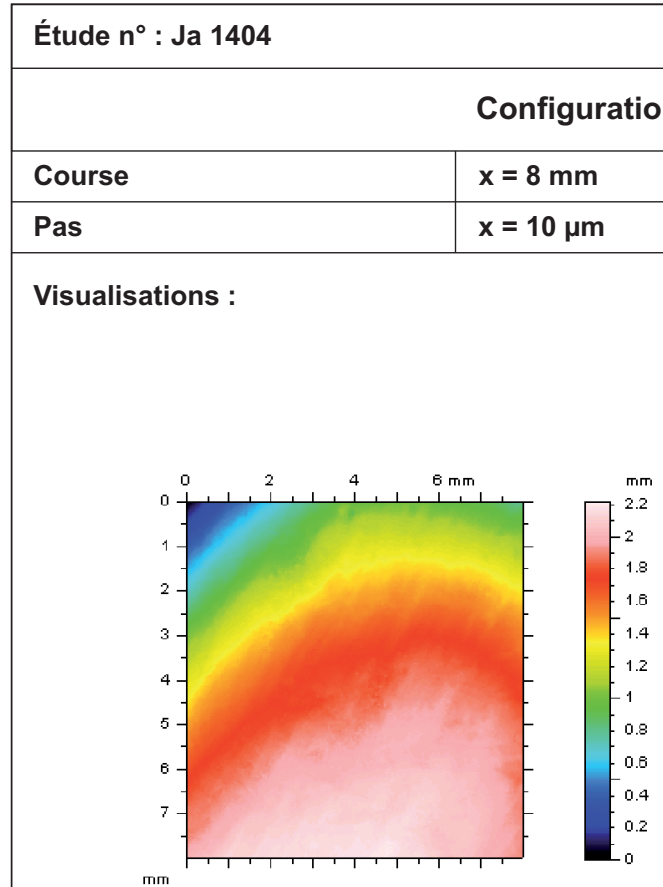

Planche 53

\begin{tabular}{|l|l|l|}
\hline \multicolumn{2}{|c|}{ Configuration d'acquisition } \\
\hline Course & $x=8 \mathrm{~mm}$ & $y=8 \mathrm{~mm}$ \\
\hline Pas & $x=10 \mu \mathrm{m}$ & $y=10 \mu \mathrm{m}$ \\
\hline
\end{tabular}

Cadence : $300 \mathrm{~Hz}$ Crayon : $\mathbf{3 0 0 0} \boldsymbol{\mu m}$

Position(s) :

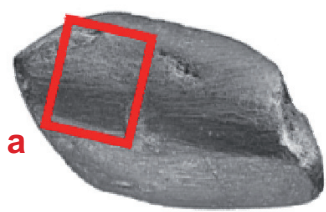

a

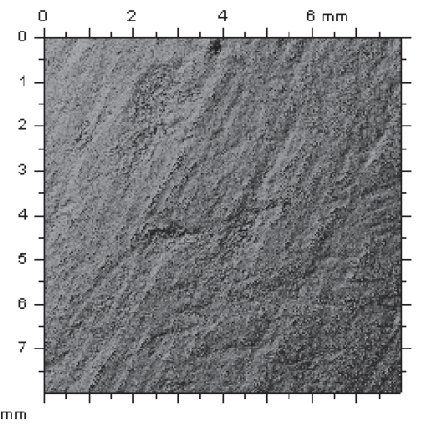

Alpha $=174^{\circ} \quad$ Beta $=36^{\circ}$
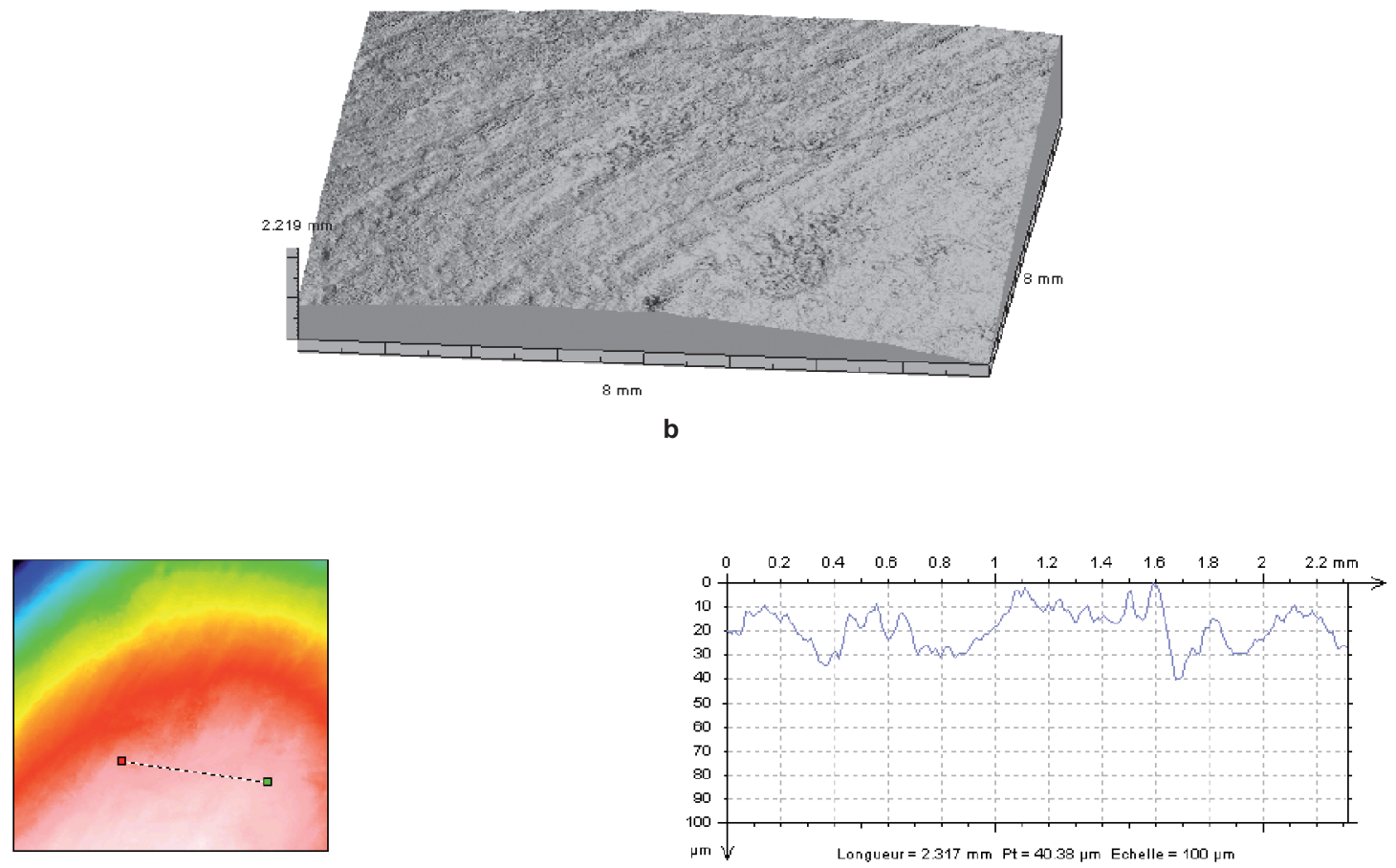

C

a. fichier de base et simulation photo

b. simulation photo en bloc 3D

c. profil : emplacement et profil 


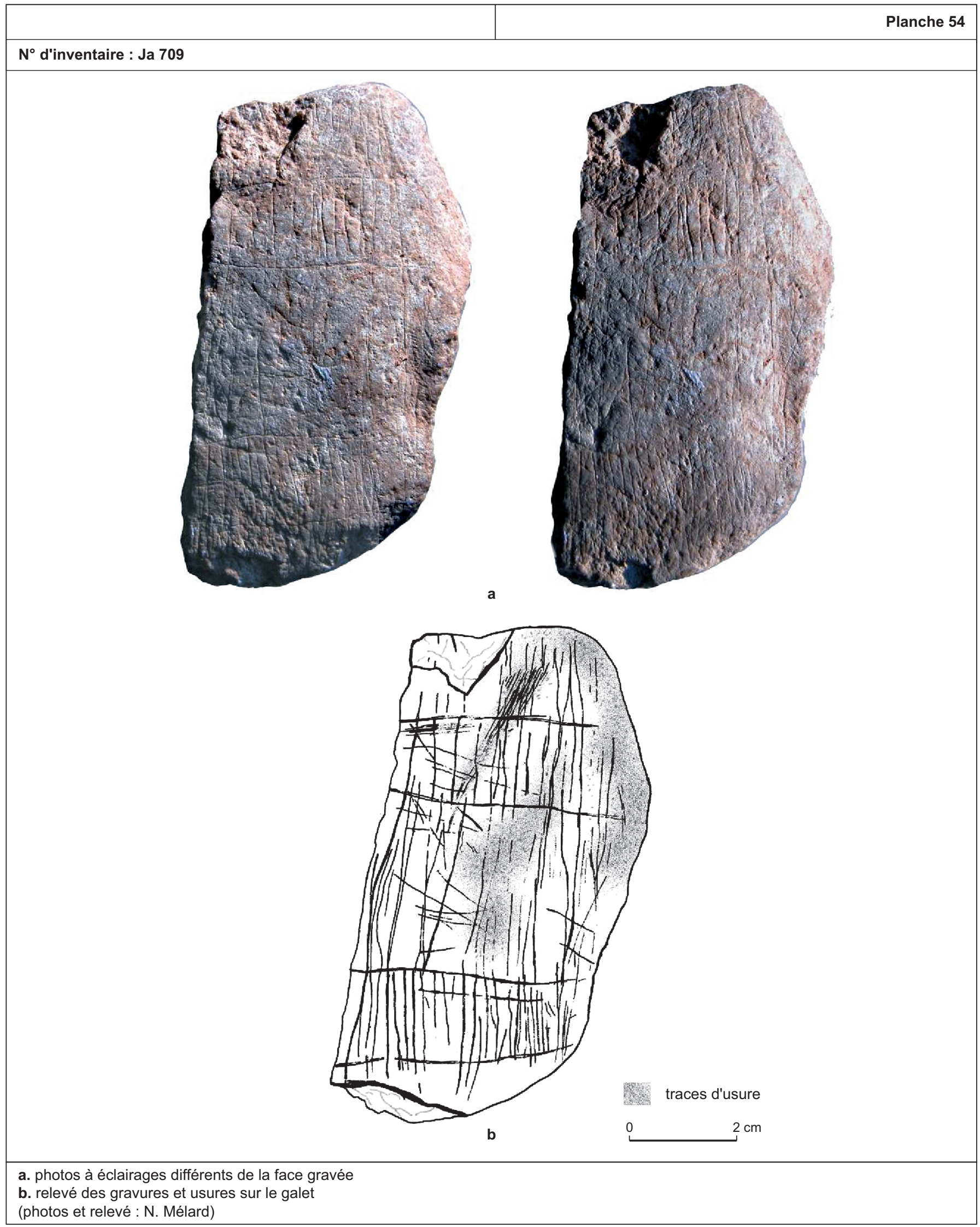




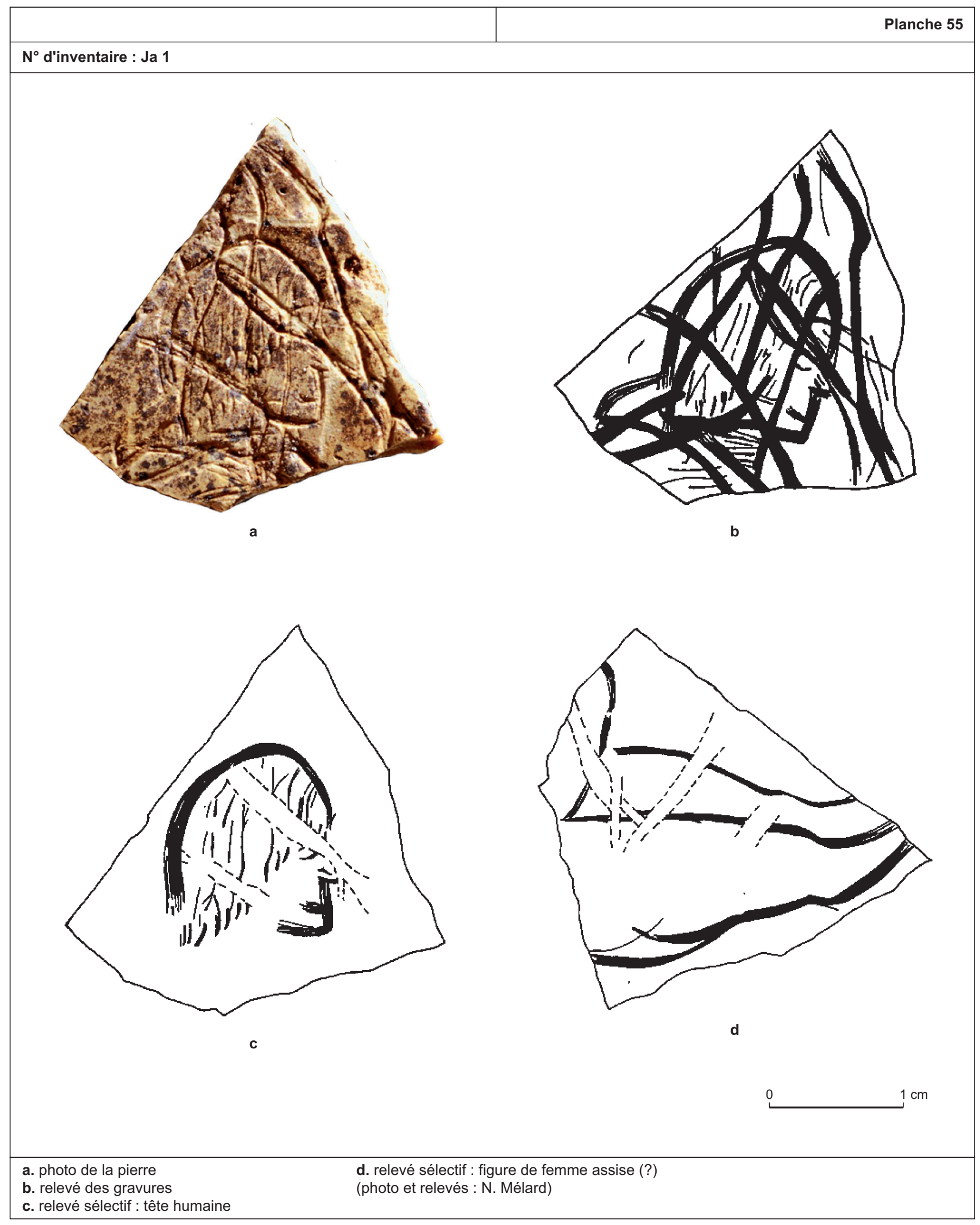




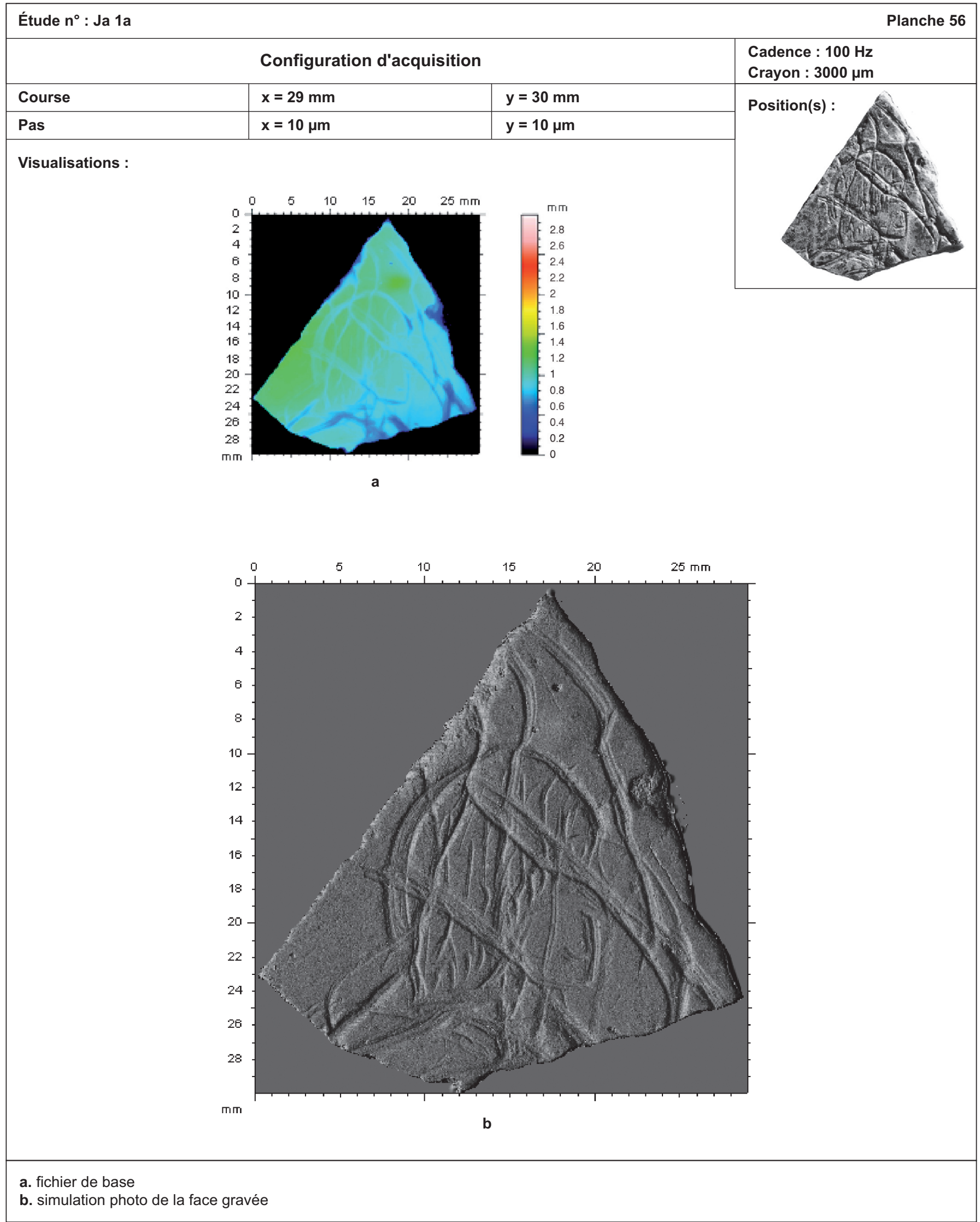

